\title{
Multimodal TMS
}

Finding biomarkers for epilepsy

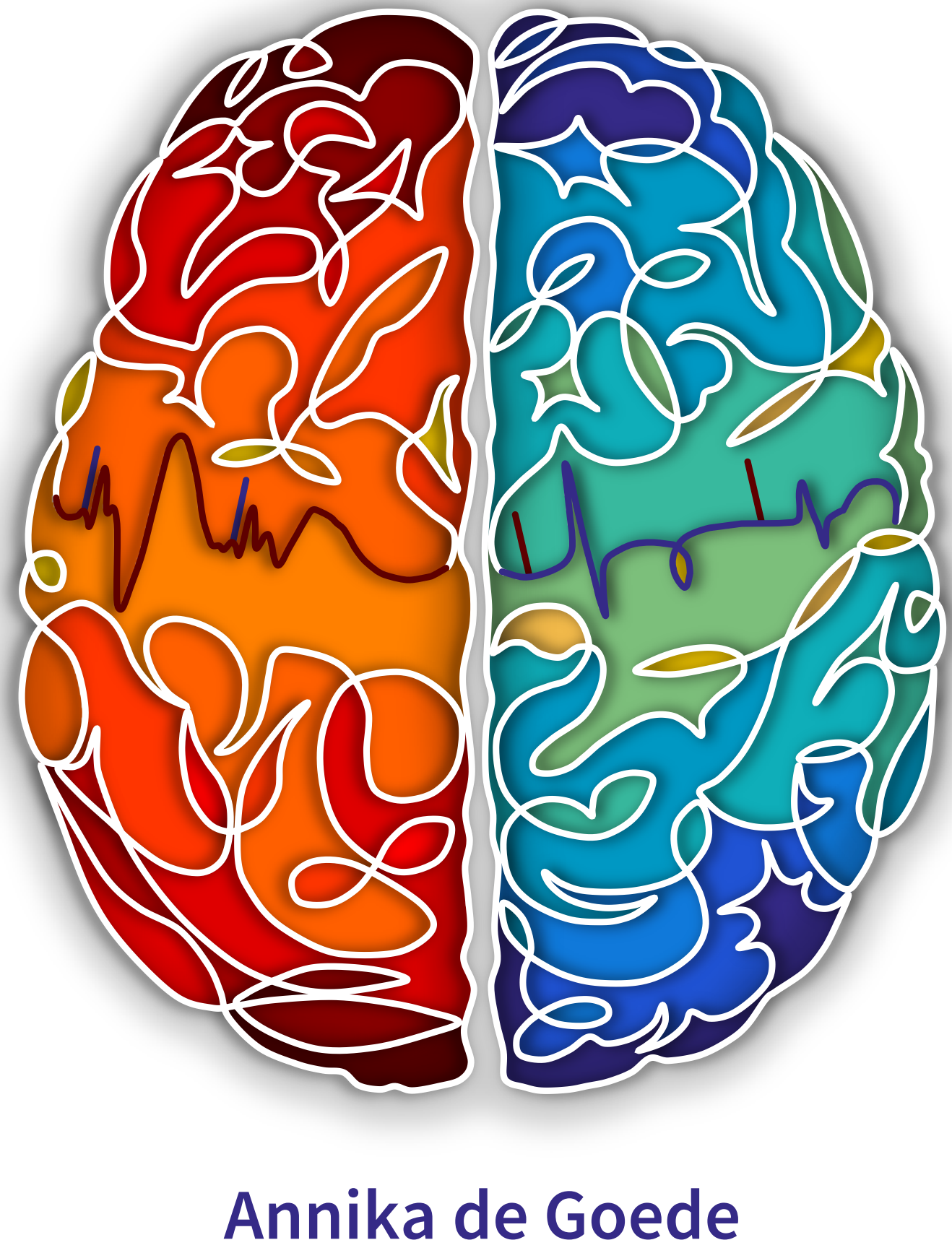





\section{MULTIMODAL TMS \\ FINDING BIOMARKERS FOR EPILEPSY}

Annika de Goede 



\title{
MULTIMODAL TMS \\ FINDING BIOMARKERS FOR EPILEPSY
}

\author{
PROEFSCHRIFT
}

\author{
ter verkrijging van \\ de graad van doctor aan de Universiteit Twente, \\ op gezag van de rector magnificus, \\ prof. dr. T.T.M. Palstra, \\ volgens besluit van het College voor Promoties \\ in het openbaar te verdedigen \\ op woensdag 27 maart 2019 om 14:45 uur
}

door

\section{Annika Aurora de Goede}

geboren op 8 april 1989

te Wageningen, Nederland 
This thesis has been approved by the promotor

prof. dr. ir. M.J.A.M. van Putten

\section{MULTIMODAL TMS - FINDING BIOMARKERS FOR EPILEPSY}

PhD thesis Annika de Goede

Printed by Gildeprint, Enschede

ISBN 978-90-365-4734-5

DOI 10.3990/1.9789036547345

The research described in this thesis was performed at the department of Clinical Neurophysiology, Technical Medical Centre, University of Twente, Enschede and the department of Clinical Neurophysiology, Medisch Spectrum Twente, Enschede.

This research was funded by the Dutch TWIN foundation for neuromodulation; in Dutch: stichting Toegepast Wetenschappelijk Instituut voor Neuromodulatie (TWIN).

The author gratefully acknowledges financial support for the publication of this thesis by Clinical Science Systems and the Dutch TWIN foundation for neuromodulation.

(c) 2019 Annika de Goede, the Netherlands. All rights reserved. No parts of this thesis may be reproduced, stored in a retrieval system or transmitted in any form or by any means without permission of the author. 


\section{Graduation committee}

Chairman and secretary

prof. dr. J.L. Herek

Promotor

prof. dr. ir. M.J.A.M. van Putten

Members

prof. dr. M.P. Richardson

prof. dr. P. Boon

dr. G.J. Groeneveld

dr. F.S.S. Leijten

prof. dr. ir. P.H. Veltink

prof. dr. S.A. van Gils
University of Twente

University of Twente

King's College London

Ghent University

Centre for Human Drug Research

University Medical Center Utrecht

University of Twente

University of Twente 



\section{Contents}

1 General introduction $\quad 1$

2 Repeatability of long intracortical inhibition in healthy subjects $\quad 11$

3 Accurate coil positioning is important for single and paired pulse TMS on the subject level 29

4 Spatiotemporal dynamics of single and paired pulse TMS-EEG responses 51

5 Infraslow activity as a potential modulator of cortical excitability 69

6 Single and paired pulse transcranial magnetic stimulation in drug naïve epilepsy 89

7 Long-interval intracortical inhibition as biomarker for epilepsy: a transcranial $\begin{array}{ll}\text { magnetic stimulation study } & 117\end{array}$

8 Multimodal TMS has the potential to improve the diagnostic process in epilepsy 139

9 General discussion $\quad 157$

$\begin{array}{ll}\text { References } & 169\end{array}$

$\begin{array}{ll}\text { List of abbreviations } & 189\end{array}$

$\begin{array}{ll}\text { English summary } & 191\end{array}$

$\begin{array}{ll}\text { Nederlandse samenvatting } & 195\end{array}$

$\begin{array}{ll}\text { Suomenkielinen yhteenveto } & 199\end{array}$

$\begin{array}{ll}\text { Dankwoord } & 203\end{array}$

$\begin{array}{ll}\text { List of publications } & 207\end{array}$

$\begin{array}{ll}\text { Conference contributions } & 209\end{array}$ 

General introduction 


\subsection{Epilepsy}

A balance between excitation and inhibition is essential for normal physiological brain function. In epilepsy, a disturbed balance results in an enduring predisposition to generate epileptic seizures (Fisher et al., 2005, 2014). Both the physical and mental discomfort associated with the seizures can have a major impact on everyday activities. In rare cases, seizures may even cause death (Nei and Bagla, 2007). Also, during seizure-free periods, epilepsy can affect the quality of life. Patients lose control over their life and might experience cognitive problems (e.g. memory loss), social problems (stigma and isolation) and psychological problems (depressive symptoms and anxiety) (Fisher et al., 2005; Quintas et al., 2012). With an estimated 70 million people affected worldwide, epilepsy is one of the most common neurological diseases (Moshé et al., 2015; Ngugi et al., 2010, 2011).

Our ability to move, think, observe and learn largely depends on a complex interaction between activation and deactivation of various brain regions. Epileptic seizures are the result of abnormal excessive or synchronous neuronal activity (Fisher et al., 2005, 2014). Patients have an increased cortical excitability, defined as the strength of a particular cortical output in response to a defined input stimulus (Bauer et al., 2014). Based on the initial manifestations, seizures are classified into those with a focal, generalized or unknown onset (Fisher et al., 2017), while etiology varies from a genetic, infectious, metabolic, immune, structural or unknown origin (Scheffer et al., 2017). Most seizures are unprovoked and occur unexpectedly, even though they can be provoked by conditions such as a concussion, fever or alcohol withdrawal (Fisher et al., 2014). Around $5 \%$ of the population experiences an unprovoked seizure during their lifetime (Forsgren et al., 1996; Hauser et al., 1993; Sander and Shorvon, 1996). However, less than half of these people will actually develop epilepsy (Berg, 2008; Bouma et al., 2016). The diagnosis of epilepsy includes 1 ) at least two unprovoked seizures more than 24 hours apart, or 2) one unprovoked seizure with an increased risk of recurrence, or 3) a diagnosed epilepsy syndrome (Fisher et al., 2005, 2014).

In the diagnostic process, both the clinical history and electroencephalogram (EEG) are relevant, while a magnetic resonance imaging (MRI) scan is only made to detect a structural origin, such as cortical dysplasia, a tumor, stroke or brain trauma. Interictal epileptiform discharges (IEDs) in the EEG reflect a tendency to generate seizures. Additionally, the type and location of IEDs can help to classify the seizures and epilepsy syndrome (Koutroumanidis et al., 2017; Rosenow et al., 2015). However, the likelihood of occurrence varies among patients. A recent meta-analysis estimated the sensitivity and specificity of the EEG at $17.3 \%$ and $94.7 \%$ in adults and the probability of epilepsy in case of a normal routine EEG at 47\% (Bouma et al., 2016). A seizure is often experienced 
as a traumatic event and the uncertainty about recurrence after a first seizure has a psychological impact on patients (Velissaris et al., 2007). To increase the diagnostic sensitivity, additional EEG recordings are frequently required (Geut et al., 2017; King et al., 1998), which simultaneously increases the time needed for visual EEG review. Nevertheless, even repeated and prolonged EEGs do not contain IEDs in approximately $10 \%$ of the diagnosed epilepsy patients (Rosenow et al., 2015). The diagnostic process is therefore often time-consuming and labor intensive, while the sensitivity of the EEG remains limited. This motivates the search for a novel biomarker to estimate the risk of seizure recurrence.

Once diagnosed with epilepsy, the majority of patients are treated with antiepileptic drugs (AEDs). Ideally, seizure freedom is attained without the occurrence of unacceptable drug-related side effects (Kwan et al., 2009). A response to the first prescribed AED is desirable, as the chance to achieve long-term remission reduces substantially for every subsequent drug or combination of drugs (Brodie et al., 2012; Kwan and Brodie, 2000, 2001). However, patients respond differently to similar drugs in terms of efficacy and tolerance (Callaghan et al., 2011; Elger and Schmidt, 2008). Guided by seizure absence or recurrence, medication is assumed to be effective if a patient is seizure-free for at least one year (Kwan et al., 2009). Based on trial and error modified by the neurologists' experience, it can take months or even years to find the optimal treatment strategy (Engel, 2008). Eventually, approximately $30 \%$ of the patients will not respond to any AEDs and suffer from refractory epilepsy (Brodie et al., 2012). In specific situations, adjunctive treatment options are considered, such as epilepsy surgery, vagus nerve stimulation, deep brain stimulation or a ketogenic diet (Boon et al., 2018; Elger and Schmidt, 2008). A biomarker that estimates the risk of seizure recurrence might potentially also reduce the time needed to evaluate the therapeutic efficacy of AEDs and alternative treatments.

Evaluating the balance between excitation and inhibition is an option to estimate the risk of seizure recurrence. A non-invasive technique to assess cortical excitability is transcranial magnetic stimulation (TMS). If cortical excitability ultimately proves to be a reliable biomarker for epilepsy, this would greatly improve the diagnostic process and the evaluation of therapeutic efficacy.

\subsection{Transcranial magnetic stimulation}

In 1985 Barker and colleagues described a novel method to directly stimulate the brain, based on the fundamental principles of electromagnetic induction. According to the Maxwell equations, a strong pulse of electrical current in a coil induces a magnetic field oriented perpendicularly to the coil. When placed on the head of a subject, the time- 
varying magnetic field induces an electric field in the cortex. At positions where the electric field has a spatial gradient parallel to the axons, or where axons bend out of the electric field, transmembrane ionic currents are generated. If these currents are large enough, they depolarize cell membranes thereby opening voltage-gated sodium channels and initiating action potentials (Barker et al., 1985; Kobayashi and PascualLeone, 2003). Since the magnetic field rapidly attenuates when the distance to the coil increases (Hess et al., 1987; Roth et al., 1991), stimulation remains limited to the superficial cortical layers (Heller and van Hulsteyn, 1992). The size of the stimulated brain area depends on the coil, being more focal for figure-of-eight than for circular coils (Cohen et al., 1990; Jalinous, 1991; Rösler et al., 1989).

Stimulation varies from giving one pulse at a time in the single pulse TMS paradigm (Barker et al., 1985), two consecutive pulses separated by a variable interval in the paired pulse TMS paradigm (Kujirai et al., 1993; Valls-Solé et al., 1992) or a train of multiple pulses at a specific frequency in the repetitive TMS paradigm (Pascual-Leone et al., 1994). Both single and paired pulse TMS are used to assess cortical excitability, as well as to evaluate changes over time (Kobayashi and Pascual-Leone, 2003). Repetitive TMS, on the other hand, can temporarily change cortical excitability, making it suitable for therapeutic purposes (Chen et al., 1997; Pascual-Leone et al., 1994). The effect of stimulation can be evaluated clinically or by combining TMS with electromyography (EMG) and/or EEG.

Initially, only assessment of the integrated corticospinal excitability was possible using single pulse TMS-EMG. Here, the primary motor cortex is stimulated and the corresponding motor evoked potential (MEP) is measured in a peripheral target muscle on the contralateral side (Barker et al., 1985). Several characteristics of the EMG and MEP are used as excitability measures, see Figure 1.1. The resting motor threshold (rMT) is defined as the minimum stimulation intensity needed to evoke at least five reproducible MEPs out of ten pulses (Rossini et al., 2015). When the target muscle is contracted instead of relaxed, the active motor threshold (aMT) is determined (Rossini et al., 2015). MEP amplitude is the peak-to-peak amplitude of the response measured in a relaxed muscle. Lastly, the cortical silent period (CSP) is the duration of the interruption in voluntary EMG activity following a pulse in a contracted muscle (Calancie et al., 1987; Fuhr et al., 1991).

Paired pulse TMS-EMG gives more insight into the cortical excitability by investigating the relative contribution of excitatory and inhibitory networks. It depends on the interstimulus interval (ISI) between the conditioning and test pulse, whether the conditioning pulse enhances or attenuates the evoked test response compared to an unconditioned MEP (Kujirai et al., 1993; Valls-Solé et al., 1992; Ziemann et al., 


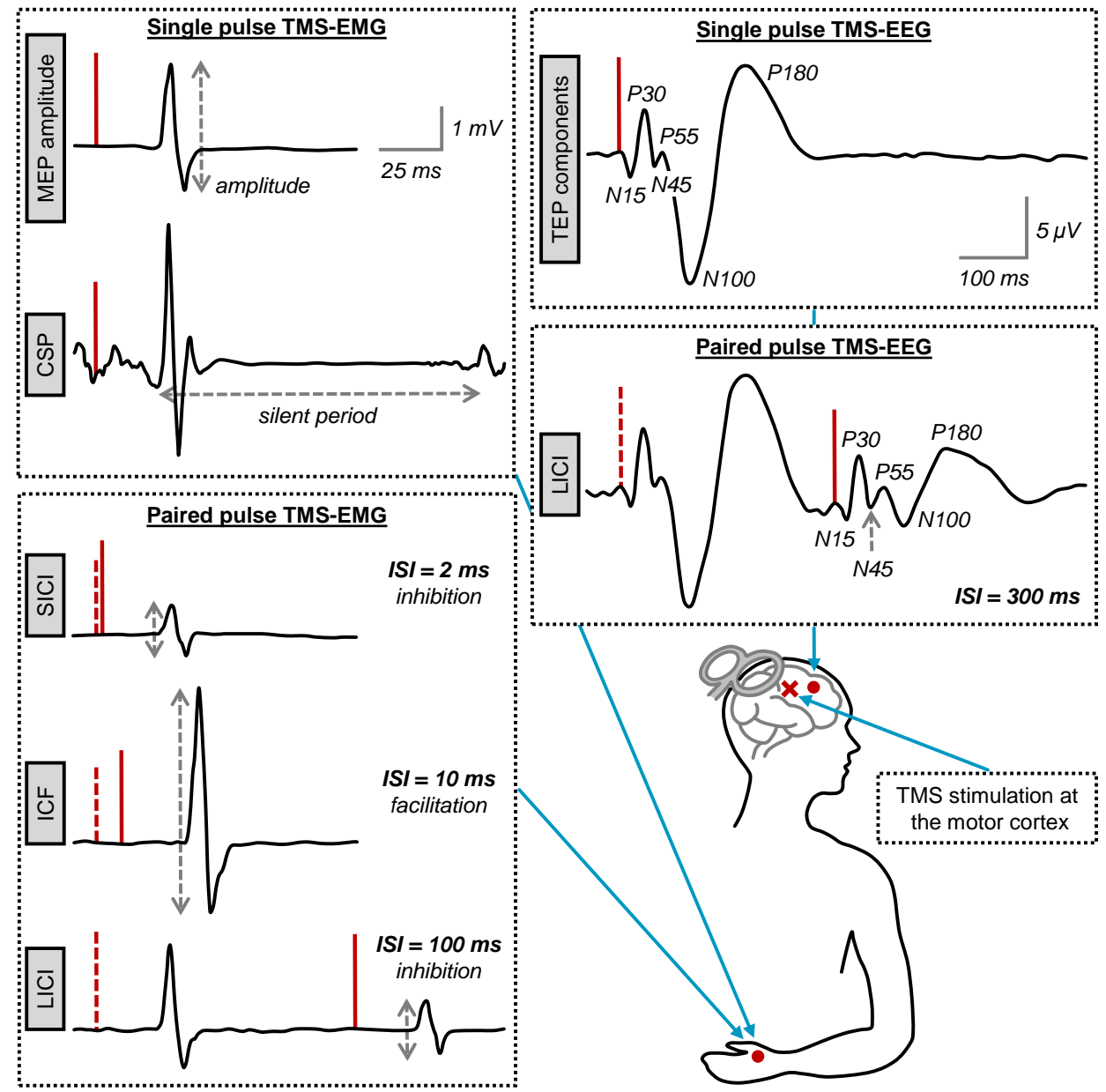

Figure 1.1: Outcome measures for TMS-EMG and TMS-EEG. Upper panels correspond to the single pulse TMS paradigm and the lower panels to the paired pulse TMS paradigm; red solid lines $=$ TMS pulse, red dashed lines $=$ conditioning TMS pulse. MEP $=$ motor evoked potential, $\mathrm{CSP}=$ cortical silent period, TEP = TMS evoked potential, $\mathrm{SICl}=$ short intracortical inhibition, ICF = intracortical facilitation, $\mathrm{LICI}=$ long intracortical inhibition and ISI = interstimulus interval. Adapted from: de Goede A.A., ter Braack E.M. and van Putten M.J.A.M. (2016). Clinical Neurophysiology, 127(9):3140-3155. 
1996b), see Figure 1.1. Short intracortical inhibition (SICI) corresponds to attenuation of the test response observed for ISIs between 1-5 ms and is associated with gammaaminobutyric acid (GABA)-A receptor mediated inhibition (Hanajima et al., 1998; Kujirai et al., 1993). Intracortical facilitation (ICF) reflects enhancement of the test response for ISIs between 6-30 ms (Kujirai et al., 1993; Ziemann et al., 1996b) and results from an imbalance between strong $N$-methyl-D-aspartate (NMDA) and weaker GABA-A receptor mediated facilitation and inhibition, respectively (Hanajima et al., 1998; Schwenkreis et al., 1999). Finally, long intracortical inhibition (LICI) corresponds to attenuation observed for ISIs between 50-400 ms (Valls-Solé et al., 1992) and is associated with GABA-B receptor mediated inhibition (McDonnell et al., 2006; Werhahn et al., 1999).

TMS-EEG enables assessment of cortical excitability by measuring the direct neuronal response in the stimulated brain (Ilmoniemi et al., 1997; Ilmoniemi and Kičić, 2010). This combination also allows stimulation and evaluation of responses of brain areas other than the commonly targeted primary motor cortex. The average response over multiple single pulses is called the TMS evoked potential (TEP). It consists of several characteristic components, namely the N15, P30, N45, P55, N100 and P180 (Ilmoniemi and Kičić, 2010; Komssi et al., 2004; Paus et al., 2001), see Figure 1.1. The amplitude of these TEP components are measures of cortical excitability. The largest amplitudes are generally recorded at the hotspot just below the coil, while they attenuate for an increase in distance to the stimulated area (Ilmoniemi and Kičić, 2010; Komssi et al., 2002). So far, the N45 and N100 components seem to be related to GABA-A and GABA-B receptor mediated inhibition, respectively (Premoli et al., 2014a).

Although paired pulse TMS-EEG makes it possible to investigate $\mathrm{SICI}, \mathrm{ICF}$ and $\mathrm{LICl}$ at the cortical level, this paradigm is not widely used. In general, the high intensity of the TMS pulse makes it technically challenging to combine TMS and EEG. Dedicated measurement equipment is needed to record EEG signals during TMS, while advanced signal processing and analysis techniques are usually required to reduce artifacts that contaminate the EEG (Ilmoniemi et al., 2015; Ilmoniemi and Kičić, 2010). In addition, interpretation of the paired pulse TEP is not straightforward, because the conditioning TEP is still ongoing when applying the test pulse. The late components of the conditioning TEP are likely affecting the early components of the TEP evoked by the test pulse. Simultaneously, these same late components of the conditioning TEP are most likely affected by applying the test pulse (Premoli et al., 2014b). However, when comparing the paired to the single pulse TEP, it seems as if the conditioning pulse modulates the paired pulse TEP components in a similar way as the MEP during paired pulse TMS-EMG, see Figure 1.1. $\mathrm{SICI}$ (ISI $2 \mathrm{~ms}$ ) is characterized by attenuation of only the late N100 and P180 components (Premoli et al., 2018), while LICI (ISIs 100 and $150 \mathrm{~ms}$ ) corresponds to suppression of almost all TEP components (Opie et al., 2017; Premoli et al., 2014b). 
Even though single and paired pulse TMS are widely used for research purposes, their clinical applicability is still limited.

\subsection{TMS in epilepsy}

Especially the combination of TMS-EMG has been used to study cortical excitability in epilepsy, with findings being the most consistent for the paired pulse paradigm. Cortical excitability was significantly increased in drug naïve new-onset epilepsy patients compared to healthy controls. Instead of $\mathrm{SICl}$ and $\mathrm{LICl}$, facilitation was found at ISIs 2, 5, 250 and 300 ms for both hemispheres in generalized epilepsy and for the hemisphere ipsilateral to the epileptic focus in focal epilepsy (Badawy et al., 2007, 2013a,d,e). Even in patients with an unprovoked seizure without recurrence, cortical excitability was significantly increased at ISIs 250 and 300 ms, although not as prominent as in the new-onset epilepsy patients (Badawy et al., 2014b). Furthermore, prescribing AEDs resulted in a decrease in cortical excitability in patients who became seizure-free, regardless of mono- or dual therapy. This change was already measurable 4-16 weeks after the first intake. In contrast, cortical excitability remained increased in refractory patients not responding to AEDs (Badawy et al., 2010a, 2013a). These differences have only been reported for subject groups and not at the level of individual patients. Nevertheless, findings suggest that increased cortical excitability could be an indicator of seizure recurrence, both in first seizure patients as well as in epilepsy patients starting with AEDs.

Although this looks promising, there are two points of concern. First, all this evidence was provided by Badawy and colleagues. We are not aware of reproducibility studies published by other groups. Even though they seem to confirm their findings repeatedly for different types of epilepsy, studies turned out to be less independent than suggested. After critical review of the papers, we published a letter to the editor expressing our concerns (Bauer et al., 2017). In their reply, the authors admitted that data of patients and controls was indeed frequently reused (Badawy et al., 2017). Second, others recently reported contrasting findings when evaluating patients who were treated with AEDs for months to years. Compared to healthy controls and drug naïve patients, cortical excitability was significantly decreased for ISIs 200-250 ms, while no differences were found between controls and drug naïve patients. After stratification on AED type, the decrease seemed related to lamotrigine, while no differences were found for valproate (Silbert et al., 2015). Thus, it is important to realize that AEDs can significantly influence the cortical excitability (Ziemann et al., 2015). Another study found decreased cortical excitability in poorly controlled patients compared to healthy controls for ISI $12 \mathrm{~ms}$, which could not be explained by AED 
treatment or epilepsy duration. However, no significant differences were reported for moderately controlled patients, nor for SICI and LICI (Pawley et al., 2017).

The combination of TMS-EEG has only been used to study chronic epilepsy patients on AEDs. The conflicting findings for single pulse TMS are likely due to the use of AEDs and differences in epilepsy syndromes. Compared to controls, the N100 and P180 amplitudes were significantly larger in a heterogeneous group of epilepsy patients (ter Braack et al., 2016), while both were decreased in progressive myoclonus epilepsy type 1 (EPM1) (Julkunen et al., 2013). Furthermore, juvenile myoclonic epilepsy (JME) patients showed a significantly larger increase in N100 and P180 amplitudes after sleep deprivation than healthy controls (Del Felice et al., 2011). Two other studies found an increase in late EEG activity around 300-1000 ms, when stimulating brain areas outside the motor cortex in focal epilepsy (Shafi et al., 2015; Valentin et al., 2008). These late responses were never observed in healthy controls and they sometimes resembled IEDs. In a similar way, paired pulse TMS was used to induce IEDs in generalized epilepsy patients (Kimiskidis et al., 2017). When focusing on the TEP, larger amplitudes were reported for the single (N30 and N100) and paired pulse (N100) components in patients than controls. However, these differences did not reach statistical significance. In addition, feature selection methods combined with a Bayesian classifier were used to differentiate between generalized epilepsy patients and healthy controls (diagnostic accuracy of 0.92) and between AED responders and non-responders (diagnostic accuracy of 0.80) (Kimiskidis et al., 2017).

Both paired pulse TMS-EMG and TMS-EEG are promising paradigms to estimate risk of seizure recurrence in epilepsy patients. Combining the various TMS paradigms has promise to provide complementary information about cortical excitability. Even though such a multimodal TMS approach has not been used before in epilepsy, it might enable diagnosis at the level of an individual patient.

\subsection{Research objectives}

This thesis describes our first steps towards the implementation of a multimodal TMS approach in epilepsy, with the aim to improve the diagnostic process. We explore the potential of single and paired pulse TMS-EMG-EEG to assess the presumed abnormal cortical excitability in epilepsy patients.

Our first research objective is to evaluate the clinical feasibility of multimodal TMS in epilepsy. In order to detect changes in cortical excitability, it is important to validate the repeatability of TMS-EMG and TMS-EEG measures. Even though neuronavigation is often recommended to ensure accurate positioning of the TMS coil, manual coil positioning is still commonly used and preferred in clinical practice, as it is easier and 
faster. To establish potential limitations of manual positioning, insight must be gained into the accuracy required during single and paired pulse TMS, as well as into the effects of small changes in positioning. In the end, the clinical feasibility largely depends on the stability and robustness of multimodal TMS.

As a second research objective, we aim to identify biological modulators of cortical excitability since trial-to-trial variations may result from endogenous fluctuations in excitability. Currently, the clinical applicability of TMS is mainly limited by the large intra- and inter-subject variability of excitability measures.

Finally, our third research objective is to evaluate the diagnostic value of multimodal TMS in first seizure epilepsy patients. The potential to become a biomarker for epilepsy depends on the ability to distinguish epilepsy patients from healthy controls. Furthermore, differentiation between first seizure patients who are diagnosed with epilepsy afterwards and those who are not, might enable the possibility to estimate the risk of seizure recurrence. This would greatly improve the diagnostic process and probably the evaluation of therapeutic efficacy as well.

\subsection{Outline of this thesis}

In Chapters 2 to 4, we evaluate the clinical feasibility of multimodal TMS in epilepsy. Chapter 2 evaluates the repeatability of paired pulse TMS-EMG, using both manual and robot-guided coil positioning. Chapter 3 investigates the accuracy of function-guided navigation for determining the motor hotspot. In addition, we evaluate the effect of a change in coil location and orientation during single and paired pulse TMS-EMG. Chapter 4 evaluates the spatiotemporal dynamics of single and paired pulse TMS-EEG in healthy subjects, as well as the repeatability and stability of TMS-EEG. In Chapter 5, we focus on candidate mechanisms involved in fluctuations of cortical excitability by exploring whether infraslow EEG activity $(<0.1 \mathrm{~Hz})$ modulates cortical excitability. In Chapters 6 to 8 , we evaluate the diagnostic value of multimodal TMS in epilepsy. Chapter 6 provides a systematic overview of the current single and paired pulse TMS findings in drug naïve epilepsy patients. Chapter 7 evaluates differences between healthy controls and refractory epilepsy patients using combined paired pulse TMSEMG data measured in four centers. Chapter 8 explores the potential of multimodal TMS to improve the diagnostic process, by evaluating differences between healthy controls, first seizure patients diagnosed with epilepsy and first seizure patients who were not diagnosed with epilepsy. Chapter 9 summarizes and discusses the main findings of this thesis and provides suggestions for future research. 



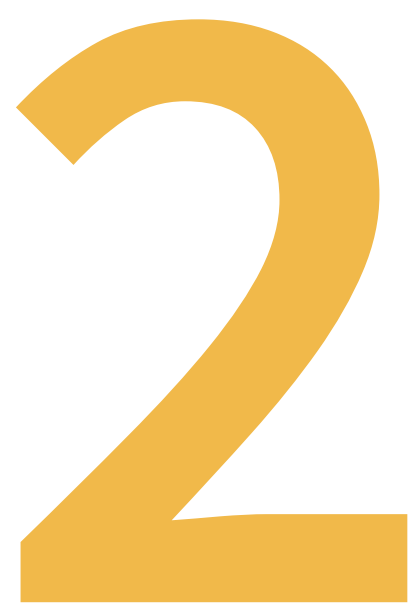

\title{
Repeatability of long intracortical inhibition in healthy subjects
}

\author{
A.A. de Goede and M.J.A.M. van Putten \\ Clinical Neurophysiology Practice 2017;2:26-34
}




\section{Abstract}

Objective: Transcranial magnetic stimulation (TMS) is widely used to assess cortical excitability. To detect changes in excitability with longitudinal studies, it is important to validate the repeatability of excitability measures within a subject between different sessions. Repeatability studies on long intracortical inhibition ( $\mathrm{LICl}$ ) are limited and reported agreement ranges from poor to good. This study aims to evaluate the repeatability of $\mathrm{LICl}$ in healthy subjects using paired pulse TMS. In addition, it investigates whether LICI repeatability differs for manual and robot-guided coil positioning.

Methods: Thirty healthy subjects (10 males, mean age $28.4 \pm 8.2$ years) were studied twice, approximately one week apart. Both motor cortices were stimulated with 50 paired pulses (intensity $120 \%$ of resting motor threshold) at interstimulus intervals (ISIs): 50, 100, 150, 200, 250 and 300 ms. In twenty subjects a figure-of-eight coil was positioned and held in place manually during both sessions, while in ten subjects a robot-navigated arm was used. $\mathrm{LICl}$ repeatability was assessed using the intraclass correlation coefficient (ICC).

Results: For manual and robot-guided coil positioning we found a large variation in repeatability at the subject and ISI level, ranging from poor to good agreement. On a group level, we found good repeatability for averaged $\mathrm{LICl}$ curves (manual: ICC $=0.91$, robot-guided: $I C C=0.95)$, which decreased when individual curves were correlated between sessions (manual: ICC $=0.76$, robot-guided: $I C C=0.84$ ).

Conclusion: For a correct interpretation of longitudinal study outcomes it is important to know the subject specific $\mathrm{LICI}$ repeatability and to analyze each ISI individually. Furthermore, the added value of robot-guided coil positioning for paired pulse TMS seems limited.

Significance: The large variation in LICI repeatability at the subject and ISI level should be taken into account in longitudinal studies, while robot-guided coil positioning seems unnecessary. 


\subsection{Introduction}

Since the introduction of transcranial magnetic stimulation (TMS) in 1985 as a method to directly stimulate the human motor cortex (Barker et al., 1985), TMS has been widely used to assess cortical excitability (Ferreri and Rossini, 2013; Kujirai et al., 1993; Valls-Solé et al., 1992). Single pulse TMS measures the global excitability of cortical interneurons, corticospinal pathways and spinal motor neurons (Abbruzzese and Trompetto, 2002; Valls-Solé et al., 1992), where paired pulse TMS focuses more on the excitability of cortical neurons only (Abbruzzese and Trompetto, 2002; Kujirai et al., 1993). By varying the interval between the paired pulses, information can be obtained from excitatory and inhibitory networks. Short intracortical inhibition (SICI) is observed when applying a sub-threshold conditioning pulse 1-5 ms before a supra-threshold test pulse (Kujirai et al., 1993). Increasing this interstimulus interval (ISI) to 6-30 ms results in intracortical facilitation (ICF) (Kujirai et al., 1993; Ziemann et al., 1996b). Long intracortical inhibition (LICI) occurs when applying a suprathreshold conditioning pulse $50-400$ ms before a supra-threshold test pulse (VallsSolé et al., 1992). It is thought that suppression and facilitation of the test response arises from inhibitory and excitatory mechanisms at the level of the cerebral cortex rather than the spinal cord (Di Lazzaro et al., 1998b; Hanajima et al., 1998; Kujirai et al., 1993). Gamma-aminobutyric acid (GABA)-A receptor mediated inhibitory mechanisms are likely to contribute to SICI (Hanajima et al., 1998; Ilić et al., 2002; Kujirai et al., 1993), GABA-B receptor mediated inhibition to LICI (McDonnell et al., 2006; Werhahn et al., 1999) and strong $N$-methyl-D-aspartate (NMDA) receptor mediated facilitation (Schwenkreis et al., 1999; Ziemann et al., 1998) combined with weaker GABA-A receptor mediated inhibition to ICF (Hanajima et al., 1998). As paired pulse TMS provides a direct measure of cortical excitability, it is a commonly used paradigm in a variety of neurological conditions like Alzheimer's disease, amyotrophic lateral sclerosis, chronic pain, epilepsy, migraine, Parkinson's disease and stroke (Chen et al., 2008; Ni and Chen, 2015).

Longitudinal studies can be used to monitor the disease process or to evaluate the effect of a (therapeutic) intervention. Individual subjects are followed over time, instead of comparing groups of subjects at a specific moment in time as in transversal (cross-sectional) studies (Badawy et al., 2012; Kimiskidis et al., 2004). At present, a transversal design can only differentiate patients from healthy subjects at a group level, due to the high inter-subject variability of excitability measures (Boroojerdi et al., 2000; Du et al., 2014; Orth et al., 2003; Wassermann, 2002). To detect individual changes in cortical excitability with a longitudinal design, it is important to validate the repeatability of excitability measures within the same subject between different TMS sessions (Badawy et al., 2012; Fleming et al., 2012; Hermsen et al., 2016). Although 
the inter-session variability is lower than the inter-subject variability (Boroojerdi et al., 2000; Du et al., 2014; Orth et al., 2003; Wassermann, 2002), mixed degrees of repeatability are found in healthy subjects for paired pulse TMS. Studies assessing SICl and ICF reported low to moderate inter-session variability (Badawy et al., 2012; Boroojerdi et al., 2000; Orth et al., 2003). Furthermore, test-retest reliability varied from moderate to good for SICI and from poor to good for ICF (Du et al., 2014; Fleming et al., 2012; Hermsen et al., 2016; Maeda et al., 2002).

Repeatability studies on LICl are limited. Initially, Farzan et al. (2010) reported high testretest reliability and Badawy et al. (2012) low inter-session variability for $\mathrm{LICl}$. However, recently Du et al. (2014) found poor reliability for long ISIs (30-500 ms) when applying a sub-threshold conditioning pulse followed by a supra-threshold test pulse. Although $\mathrm{LICl}$ is generally induced by two supra-threshold pulses, this latter study indicates that repeatability of $\mathrm{LICI}$ might not be as optimal as initially shown and needs to be further investigated.

Inter-session variability observed in longitudinal studies is assumed to be due, at least partially, to inaccuracies in positioning and handling of the TMS coil. Navigation methods can be used to ensure accurate coil positioning within and between consecutive sessions (Lefaucheur, 2010). The easiest and most conventional method of coil positioning is to use signature outputs, like motor responses or phosphenes, to identify the cortical area for stimulation (Barker et al., 1985; Lefaucheur, 2010; Rossini et al., 2015). More accurate methods include robot-guided positioning or stereotaxic neuronavigation (Lefaucheur, 2010; Sparing et al., 2008).

In this study we evaluate the repeatability of $\mathrm{LICl}$ in healthy subjects using paired pulse TMS. In addition, we investigate whether LICI repeatability differs for manual and robotguided positioning of the TMS coil.

\subsection{Materials and methods}

The study protocol (trial ID: NL49854.044.14) was approved by the local medical ethics committee (Medisch Spectrum Twente, Enschede, the Netherlands) and was in accordance with the Declaration of Helsinki ( $64^{\text {th }}$ WMA General Assembly, Fortaleza, Brazil, October 2013). We followed the guidelines for the use of TMS in clinical practice and research (Rossi et al., 2009).

\subsubsection{Subjects}

Healthy adults (18 years or older) with no personal history of epilepsy or brain lesion(s) were included. Subjects were excluded if they were taking pro-epileptogenic medication, had implanted devices (cochlear implant or deep brain stimulator), had 
metal objects in their brain or skull, or had a history of spinal cord surgery. In addition, females were excluded if there was a possibility of pregnancy.

All included subjects gave written informed consent and filled out the Screening Questionnaire before TMS (Rossi et al., 2011) and the Dutch Handedness Questionnaire (van Strien, 1992, 2003).

\subsubsection{TMS protocol}

Subjects were seated comfortably in a chair, with their hands pronated in a relaxed position. They were asked to keep their eyes open and their head in a fixed position. Subjects underwent the same TMS session twice, under equal circumstances: same investigators, measurement set-up and moment of the day. The second session took place approximately one week later (mean 7.5 days; range 6-15 days).

Paired biphasic TMS pulses, with a pulse duration of $400 \mu$ s, were given by a Magstim Rapid $^{2}$ Stimulator (The Magstim Company Ltd, Whitland, United Kingdom). Both motor hotspots of the abductor digiti minimi (ADM) muscle were stimulated at each ISI with 50 paired pulses. We always started at the left hemisphere, after which the right hemisphere was stimulated. At each side, we first randomly applied ISIs 200, 250 and $300 \mathrm{ms,}$ followed by ISIs 50, 100 and $150 \mathrm{~ms}$ in a random order. Because of technical limitations most subjects of the robot-guided coil positioning group could not be stimulated at ISI $50 \mathrm{~ms}$. A random interval of approximately $4 \mathrm{~s}$ (range 3.5-4.5 s) was kept between pairs of consecutive pulses. Both the conditioning and test pulse were given at an intensity of $120 \%$ the resting motor threshold (rMT). rMT was defined as the minimum stimulation intensity needed to induce at least five motor evoked potentials (MEPs), with a peakto-peak amplitude of at least $50 \mu \mathrm{V}$, out of ten consecutive pulses (Groppa et al., 2012; Rossini et al., 2015).

\subsubsection{Coil positioning}

The figure-of-eight air film $70 \mathrm{~mm}$ coil (The Magstim Company Ltd, Whitland, United Kingdom) was placed tangentially with the handle pointing backwards and laterally at an angle of $45^{\circ}$ from the midline. In twenty subjects the coil was positioned and held in place manually during both sessions, always by the same investigator. In the other ten subjects, coil positioning was performed by a robot-navigated system (ANT Neuro, Enschede, the Netherlands). Subjects were tracked by a Polaris infrared camera system (Northern Digital, Waterloo, Canada), using a headband with four passive reflective markers. A head model was created using a general magnetic resonance image and by collecting three landmarks and approximately 300 additional points on the scalp with a tracking pointer. The location of the ADM hotspot was defined manually and indicated 
on the head model. A robotic arm, containing the coil, was used for positioning and displacements from the indicated location were detected and actively corrected to ensure accurate coil positioning during the entire session.

\subsubsection{Electromyography recording and analysis}

The electromyogram (EMG) was recorded, from the ADM and abductor pollicis brevis (APB) muscles, with two surface $\mathrm{Ag} / \mathrm{AgCl}$ electrodes placed in a belly-tendon montage. Although we stimulated the ADM hotspot, we simultaneously recorded the activity of the APB muscle. The ground electrode was placed on the dorsal side of the left hand. EMG was sampled at a frequency of either $2048 \mathrm{~Hz}$ (robot-guided coil positioning) or $5000 \mathrm{~Hz}$ (manual coil positioning) and recorded using an additional amplifier (TMSi, Oldenzaal, the Netherlands).

Even though subjects were asked to fully relax their ADM and APB muscles, recordings were afterwards checked for muscle pre-activation. Trials containing EMG activity larger than $50 \mu \mathrm{V}$ in the $50 \mathrm{~ms}$ preceding the conditioning pulse were excluded. If more than 25 of the original 50 repetitions were discarded, that specific ISI was not taken into account during further analysis.

The amount of inhibition was determined separately for each subject, ISI and stimulated hemisphere. First, we calculated the mean peak-to-peak amplitude of the conditioning and test response, by taking the average over the 50 (or less) repetitions. Next, we calculated the ratio between this mean amplitude of the second test response (TR) and this mean amplitude of the first conditioning response (CR), expressed as a percentage: $100 \cdot$ TR/CR (\%) (Valls-Solé et al., 1992). In each subject we ended up with two LICI ratios for each ISI: one ratio for the dominant and one ratio for the nondominant hemisphere. This ratio represents inhibition for values below $100 \%$ and facilitation for values above $100 \%$.

\subsubsection{Statistical analysis}

The intraclass correlation coefficient (ICC) was used to estimate the agreement between repeated sessions; model ICC $(3,1)$ : two-way mixed single measures, absolute agreement (Shrout and Fleiss, 1979). Repeatability of LICI was assessed on three levels: 1) ISI, 2) subject and 3) group level.

At the ISI level, we correlated the individual LICI ratios of all subjects measured at a particular ISI during the first session, with all the individual ratios from the second session. We did this for the $\mathrm{LICl}$ ratios measured at the dominant or non-dominant hemisphere only and for the ratios of both hemispheres pooled (two $\mathrm{LICl}$ ratios per subject per session). 
At the subject level, we correlated the individual LICI ratios of all ISIs measured in a particular subject during the first session, with all the individual ISI ratios from the second session. For this, we pooled the $\mathrm{LICI}$ ratios of all ISIs measured at both hemispheres for each subject (two LICI ratios per ISI per session).

At the group level, we calculated for each ISI the mean $\mathrm{LICI}$ ratio over all subjects and evaluated these averaged $\mathrm{LICl}$ curves. We correlated the mean $\mathrm{LICl}$ ratios of all ISIS measured during the first session, with the mean ratios from the second session. We did this for the mean $\mathrm{LICl}$ ratios measured at the dominant or non-dominant hemisphere only and for the mean ratios of both hemispheres pooled (two $\mathrm{LICl}$ ratios per ISI per session). Additionally, we correlated the individual LICI ratios of all ISIs measured in all subjects during the first session, with all the individual ISI ratios from the second session. Again, we did this for each hemisphere separately and for the ratios of both hemispheres pooled (two LICI ratios per ISI per subject per session).

As ISI 50 ms was not applied in most subjects from the robot-guided coil positioning group, this interval was not included into the ISI and group level analysis in this group. ICC varies between $0-1$, where 1 represents perfect repeatability. Consistent with Du et al. (2014), we considered ICC values above 0.8 as good, values from $0.6-0.8$ as moderate and values below 0.6 as poor repeatability.

\subsection{Results}

Thirty-four healthy subjects were included in this study. Four subjects were excluded from analysis: one subject was not feeling well during the first session, in one subject it was not possible to perform the second session 1-2 weeks later due to illness and two subjects had a rMT above $83 \%$ of maximum stimulator output, making stimulation at $120 \%$ rMT not possible. Except from the first excluded subject, all participants tolerated the paired pulse protocol well and no adverse events happened.

Thirty subjects (10 males, mean age $28.4 \pm 8.2$ years; range 20-51 years, 27 righthanded) completed the entire study. In twenty subjects coil positioning was performed manually, hereafter referred to as 'manual group'. Robot-guided coil positioning was applied in ten subjects, referred to as 'robot group'.

As we continuously stimulated the ADM hotspot and similar results were obtained for the ADM and APB muscles, only outcomes of the ADM muscle are presented below.

\subsubsection{Repeatability of resting motor threshold}

The averaged rMT values of the first and second session are given in Table 2.1. The ICC showed good repeatability for $\mathrm{rMT}$ between repeated sessions in both the manual 
Table 2.1: Overview of rMT values (mean \pm standard deviation (SD)) of both TMS sessions, separated for manual and robot-guided coil positioning at the dominant and non-dominant hemisphere. rMT is the percentage of maximum stimulator output $(0.8 \mathrm{~T})$.

\begin{tabular}{lcc}
\hline Coil positioning (hemisphere) & Session 1: rMT (\%) & Session 2: rMT (\%) \\
\hline Manual (dominant) & $67.9 \pm 8.5$ & $68.6 \pm 7.9$ \\
Manual (non-dominant) & $67.5 \pm 8.0$ & $66.9 \pm 7.4$ \\
Robot-guided (dominant) & $70.8 \pm 8.8$ & $69.6 \pm 11.6$ \\
Robot-guided (non-dominant) & $70.1 \pm 13.4$ & $67.9 \pm 11.8$ \\
\hline
\end{tabular}

group (dominant hemisphere: ICC $=0.86$, non-dominant hemisphere: $I C C=0.87$, overall: ICC $=0.86$ ) and robot group (dominant hemisphere: ICC $=0.92$, non-dominant hemisphere: ICC $=0.86$, overall: $I C C=0.88$ ), see Figure 2.1 .

\subsubsection{Repeatability of long intracortical inhibition}

\section{Interstimulus interval level}

Correlating, for each ISI, the inhibition ratios of all subjects measured during the first and second session showed a large variation in repeatability at the ISI level. In the manual group agreement varied from poor to moderate levels (range ICC: $0.30-0.78$ ) and in the robot group from poor to good levels (range ICC: $0.20-0.92$ ), see Table 2.2. No statistical differences in repeatability were found between the dominant and non-dominant hemisphere in both groups (manual: $p=0.83$, robot: $p=0.46$ ). When inhibition ratios of both hemispheres were pooled, repeatability was poorest for ISIs 100 and $150 \mathrm{~ms}$ (overall: ICC $=0.44$ ) in the manual group and best for ISI $50 \mathrm{~ms}$ (overall: ICC $=0.77$ ). In the robot group, repeatability was poorest for ISI $100 \mathrm{~ms}$ (overall: ICC = 0.49 ) and best for ISI $150 \mathrm{~ms}$ (overall: ICC $=0.81$ ), see Figure 2.2A.

\section{Subject level}

To assess the repeatability of $\mathrm{LICl}$ at an individual level, we pooled the inhibition ratios of all ISIs measured at both hemispheres for each subject. There was a large variation in $\mathrm{LICl}$ curves and $\mathrm{LICl}$ repeatability between subjects, see Figure 2.2B and Figure 2.3. In the manual group, $45 \%$ of the subjects showed good (ICC $>0.8$ ), $20 \%$ moderate $(0.6 \leq \mathrm{ICC} \leq 0.8)$ and $35 \%$ poor $(\mathrm{ICC}<0.6)$ repeatability. In the robot group, $50 \%$ showed good, $30 \%$ moderate and $20 \%$ poor repeatability.

\section{Group level}

The averaged $\mathrm{LICl}$ curves of all subjects for both sessions are shown in Figure 2.4, separated for both hemispheres in the manual and robot group. The $\mathrm{LICl}$ curves show great similarities; facilitation for ISI $50 \mathrm{~ms}$ and inhibition for ISIs 100-300 ms. The 


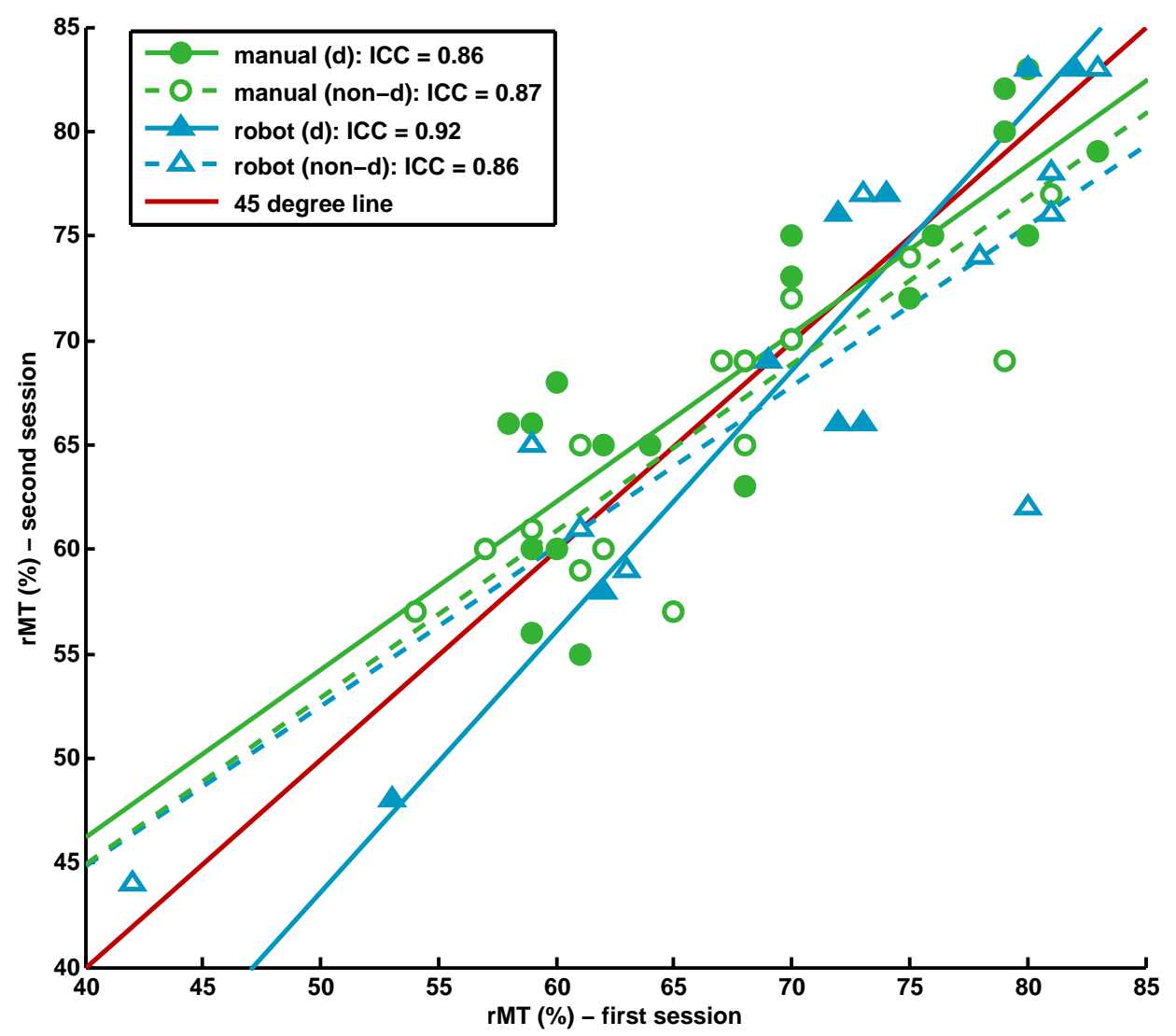

Figure 2.1: Repeatability of resting motor threshold (rMT). The intraclass correlation coefficient (ICC) showing good repeatability for rMT between the first and second session in the manual (green dots) and robot group (blue triangles); $d=$ dominant hemisphere, non- $d=$ non-dominant hemisphere. The red line represents the $45^{\circ}$ line through the origin: perfect repeatability. rMT is the percentage of maximum stimulator output $(0.8 \mathrm{~T})$. 

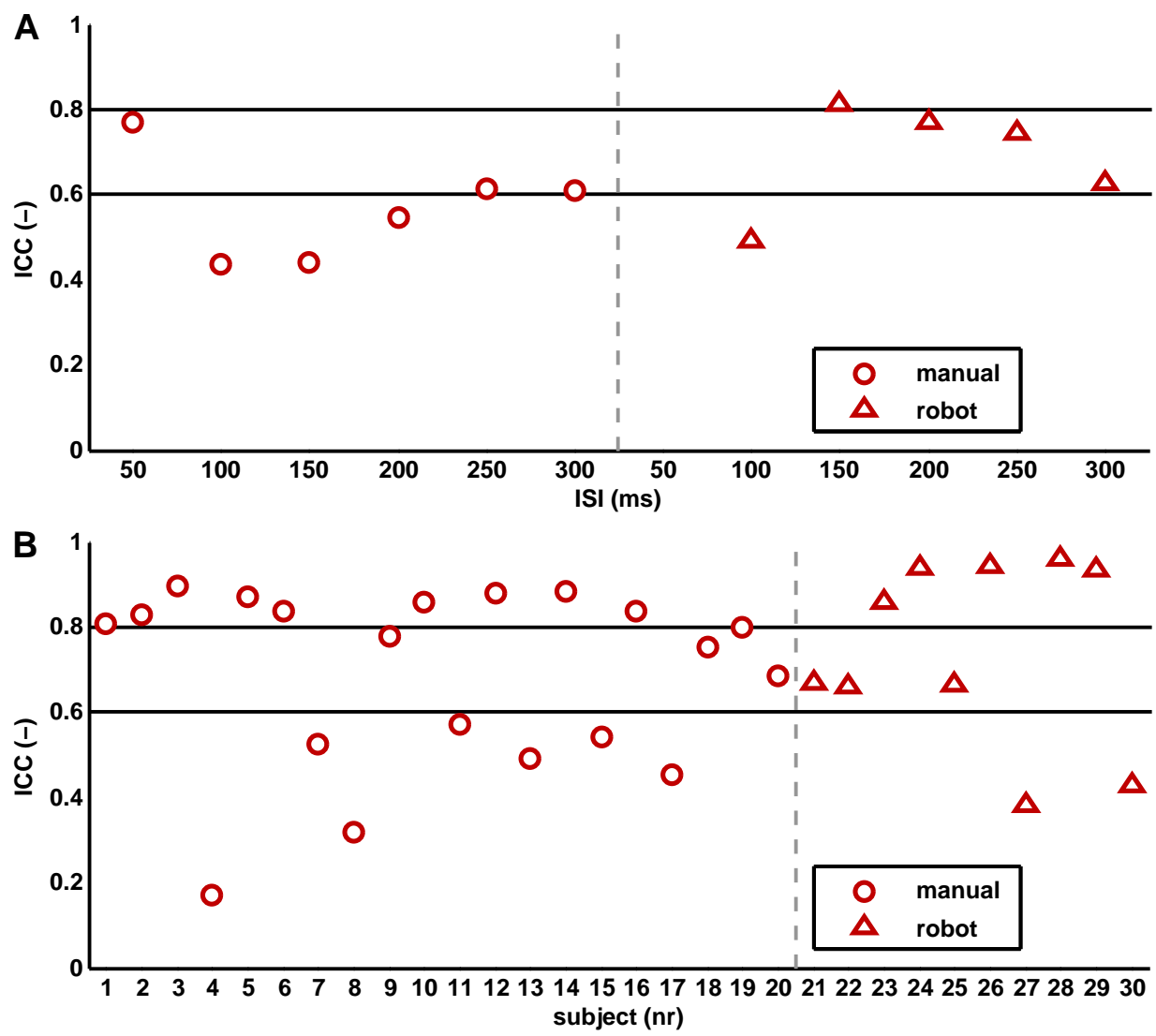

Figure 2.2: Repeatability of $\mathrm{LICl}$ at the interstimulus interval (ISI) and subject level. The intraclass correlation coefficient (ICC) showing the repeatability of inhibition ratios between the first and second session for each A) ISI and B) subject. Repeatability of the manual group is shown in red dots and of the robot group in red triangles; inhibition ratios of both hemispheres pooled. The horizontal black lines, at ICC $=0.6$ and ICC $=0.8$, represent thresholds for moderate and good repeatability. Overall, a large variation in repeatability is seen for ISIs and subjects, ranging from poor to good levels of agreement. 

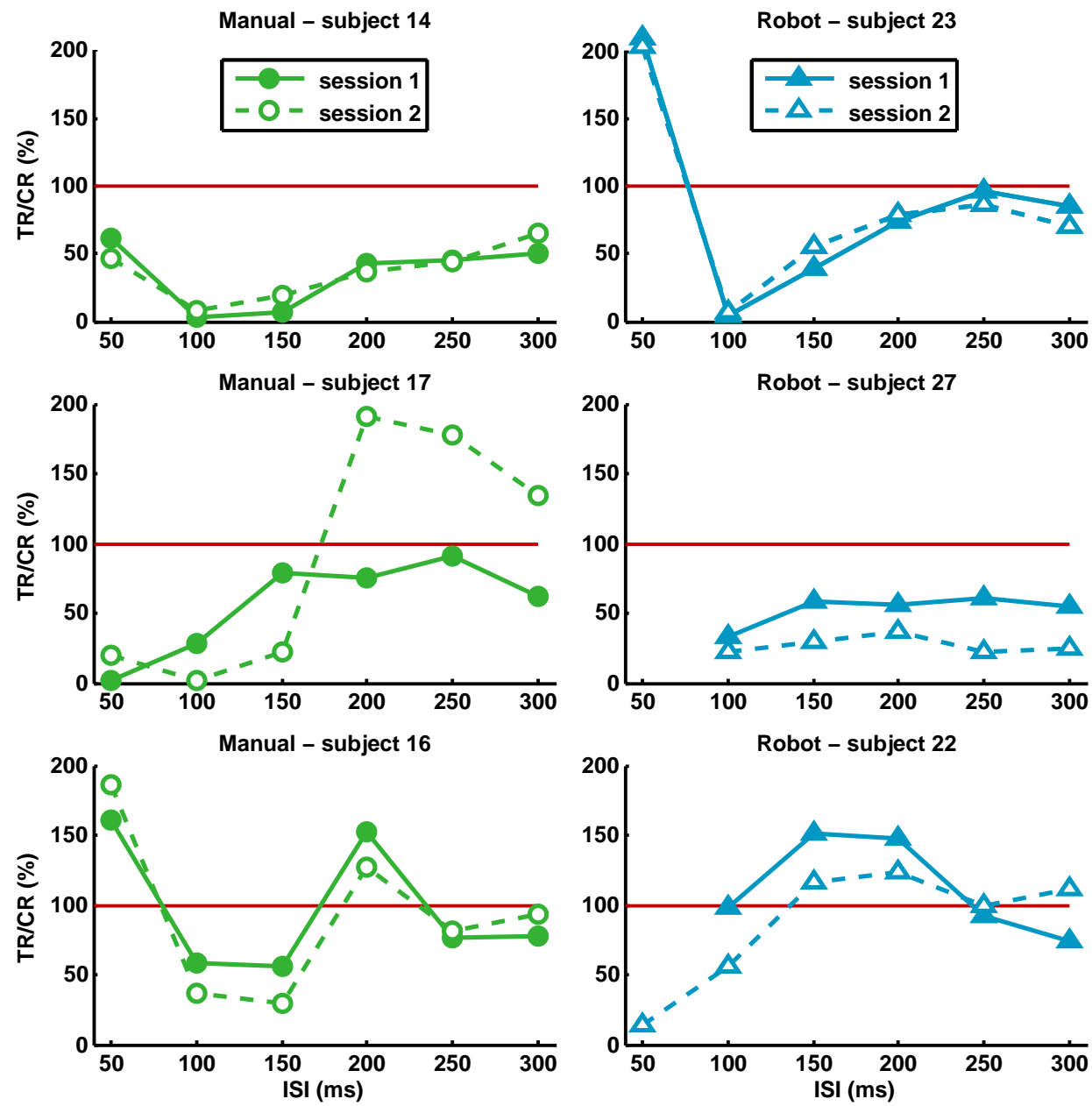

Figure 2.3: Repeatability of $\mathrm{LICl}$ at the subject level. Examples showing the large variation in repeatability between subjects of the manual (in green) and robot group (in blue). At the top, two examples of subjects where $\mathrm{LICl}$ curves showed good repeatability between both sessions. In the middle, examples of poor repeatability and at the bottom examples where $\mathrm{LICl}$ curves are vertically shifted between sessions while retaining their shape. Values below the red line $(100 \%)$ represent inhibition and values above facilitation; $T R=$ test response, $C R=$ conditioning response and $I S I=$ interstimulus interval. All examples are from the dominant hemisphere. 

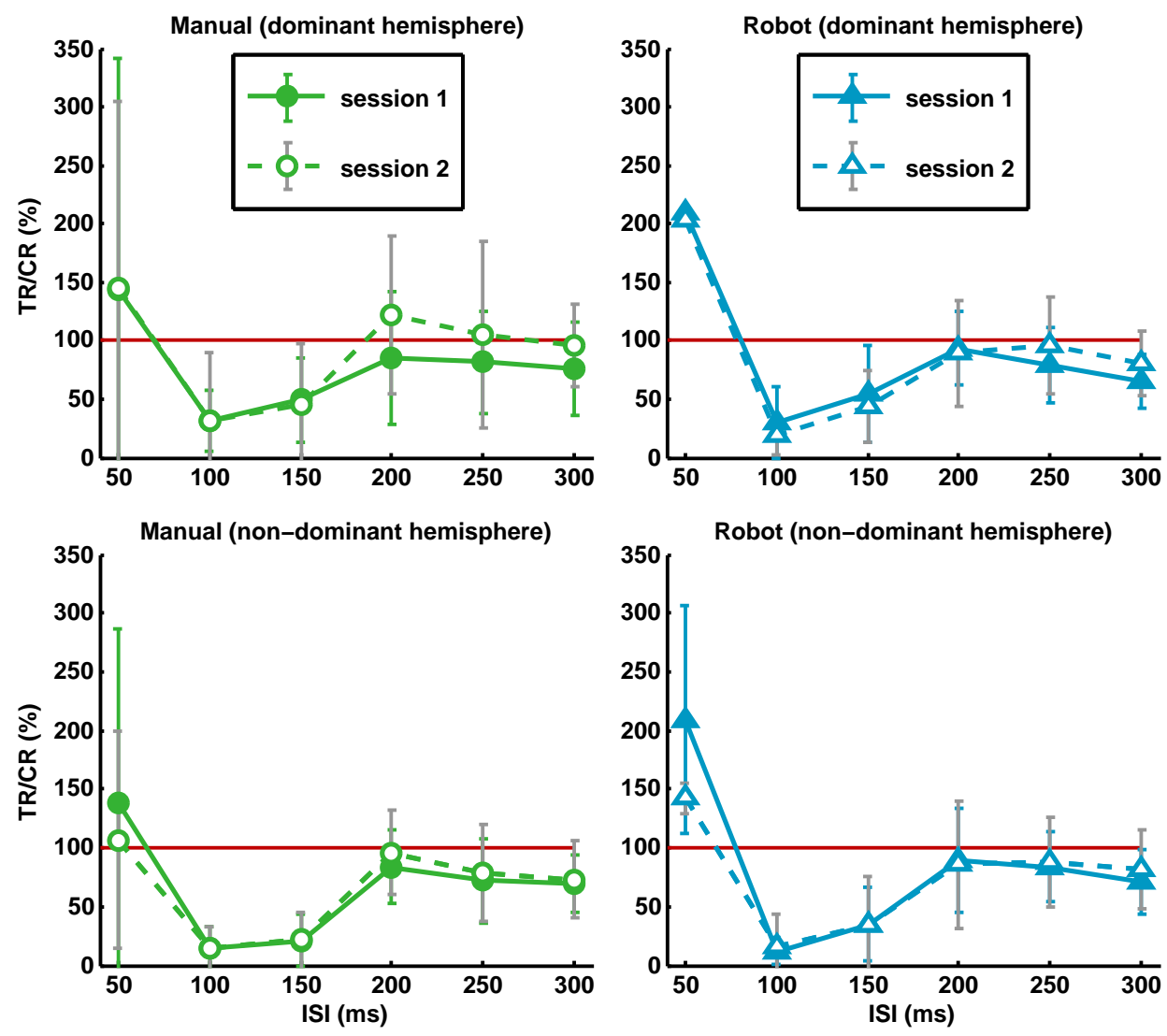

Figure 2.4: Repeatability of $\mathrm{LICl}$ at the group level. The averaged $\mathrm{LICl}$ curves (mean $\pm \mathrm{SD}$ ) of all subjects for both sessions, separated for manual (in green) and robot-guided coil positioning (in blue) at the dominant (at the top) and non-dominant hemisphere (at the bottom). Values below the red line $(100 \%)$ represent inhibition and values above facilitation; $T R=$ test response, $C R=$ conditioning response and ISI = interstimulus interval. All LICI curves showed good repeatability and great similarities; facilitation for ISI $50 \mathrm{~ms}$ and inhibition for ISIs 100-300 ms (exceptions are ISIs 200 and $250 \mathrm{~ms}$ in the manual group during the second session at the dominant hemisphere). 
Table 2.2: Overview of the intraclass correlation coefficient (ICC) showing the repeatability of inhibition ratios between the first and second session for each interstimulus interval (ISI), separated for manual and robot-guided coil positioning at the dominant and non-dominant hemisphere.

\begin{tabular}{lcccc}
\hline ISI (ms) & $\begin{array}{c}\text { Manual } \\
\text { (dominant) }\end{array}$ & $\begin{array}{c}\text { Manual } \\
\text { (non-dominant) }\end{array}$ & $\begin{array}{c}\text { Robot-guided } \\
\text { (dominant) }\end{array}$ & $\begin{array}{c}\text { Robot-guided } \\
\text { (non-dominant) }\end{array}$ \\
\hline $\mathbf{5 0}$ & 0.78 & 0.77 & - & - \\
$\mathbf{1 0 0}$ & 0.39 & 0.45 & 0.70 & 0.20 \\
$\mathbf{1 5 0}$ & 0.30 & 0.56 & 0.85 & 0.78 \\
$\mathbf{2 0 0}$ & 0.56 & 0.51 & 0.61 & 0.87 \\
$\mathbf{2 5 0}$ & 0.61 & 0.62 & 0.61 & 0.92 \\
300 & 0.66 & 0.47 & 0.38 & 0.81 \\
\hline
\end{tabular}

only exceptions are ISIs 200 and 250 ms, where slight facilitation was measured in the manual group at the dominant hemisphere during the second session. Correlating the mean $\mathrm{LICl}$ ratios of all ISIs measured during the first and second session, showed good repeatability in the manual (dominant hemisphere: ICC $=0.89$, non-dominant hemisphere: $I C C=0.94$, overall: $I C C=0.91$ ) and robot group (dominant hemisphere: ICC $=0.91$, non-dominant hemisphere: $I C C=0.98$, overall: $I C C=0.95)$. However, when individual $\mathrm{LICI}$ ratios of all ISIs measured in all subjects of each group were correlated between sessions, the manual group showed moderate (dominant hemisphere: ICC = 0.73 , non-dominant hemisphere: $I C C=0.80$, overall: $I C C=0.76$ ) and the robot group good $\mathrm{LICI}$ repeatability (dominant hemisphere: ICC $=0.81$, non-dominant hemisphere: ICC $=0.86$, overall: ICC $=0.84)$.

\subsection{Discussion}

In this study we evaluated the repeatability of $\mathrm{LICl}$ in healthy subjects using paired pulse TMS. For manual and robot-guided coil positioning we found a large variation in repeatability for individual subjects and ISIs, ranging from poor to good levels of agreement. On a group level, good repeatability was found for the averaged $\mathrm{LICl}$ curves, which decreased when individual curves were correlated between sessions. Similar results were obtained for the ADM and APB muscles. In addition, rMT showed good repeatability in both groups.

\subsubsection{Repeatability at the interstimulus interval level}

In the manual group, repeatability varied from poor to moderate levels at the ISI level. Repeatability was poorest for ISIs 100 and $150 \mathrm{~ms}$ (overall: ICC $=0.44$ ) and best for 
ISI $50 \mathrm{~ms}$ (overall: ICC $=0.77$ ). In the robot group, repeatability ranged from poor to good; poorest for ISI $100 \mathrm{~ms}$ (overall: ICC $=0.49$ ) and best for ISI $150 \mathrm{~ms}$ (overall: ICC = 0.81). These outcomes are in-between the findings by Du et al. (2014) and Badawy et al. (2012). The first study reported poor agreement for ISIs 30-500 ms; even ICC $<0.1$ for ISIs 120-500 ms (Du et al., 2014). The higher repeatability reported by us might be the result of a difference in the calculation of the inhibition ratio. We compared the test response to the conditioning response, instead of to the unconditioning single pulse response, which reflects a more direct modulation effect. Furthermore, in their study $\mathrm{LICl}$ was not induced by two supra-threshold pulses. Badawy et al. (2012) reported good agreement for ISIs 50-400 ms (range rho-c: 0.93-0.95); highest for ISI 50 ms. The fact that their outcomes were based on a group analysis, while we correlated inhibition ratios of individual subjects between sessions, seems to explain their high agreement levels. We found similar repeatability at a group level, which decreased when including the inter-subject variability at the ISI level. Although Badawy et al. (2012) reported correlation quantified by rho-c, we used ICC, as this is a more common method to estimate repeatability (Nickerson, 1997). Except for a term that decreases with increasing number of subjects, both coefficient equations are identical (Lin, 1989; Nickerson, 1997). Our data was only marginally affected when using rho-c.

The observed large variation in repeatability between ISIs indicates that it is preferred to analyze each ISI individually, instead of combining ISIs. Which repeatability for each ISI is required ultimately depends on the research question and study population. For example, in epilepsy research it appears that especially ISIs 2, 5, 250 and 300 ms differ significantly between patients and controls (de Goede et al., 2016). Therefore repeatability should be optimal for these particular ISIs, but not necessarily for the others.

We can only speculate about what causes the large variation in repeatability. Recently, Opie et al. (2017) suggested that there might be a difference between ISIs in the relative contribution of inhibitory mechanisms associated with $\mathrm{LICl}$. They speculated that activation of both pre- and post-synaptic GABA-B receptors may contribute to $\mathrm{LICl}$ at ISI $100 \mathrm{~ms}$, whereas at ISI 150 ms solely pre-synaptic GABA-B receptors are activated (Opie et al., 2017). In addition, the variation in repeatability may be partly due to habituation or loss of attention that might have occurred during the TMS session. Repeatability was especially poor for ISI $100 \mathrm{~ms}$, an interval that was applied in the second part of the session. However, better agreement levels were found for ISIs 50 and 150 ms, which were also applied during the second part.

\subsubsection{Repeatability at the subject level}

$\mathrm{LICl}$ curves and corresponding $\mathrm{LICl}$ repeatability varied widely between subjects, with repeatability ranging from poor to good. Du et al. (2014) also described a large variation 
in response profiles of healthy subjects. Despite a large variance across subjects, they stated that individuals appear to have a unique inhibition-facilitation profile that remains relatively stable during repeated sessions (Du et al., 2014). Indeed, Figure 2.3 shows examples of good repeatability (at the top) and cases where $\mathrm{LICl}$ curves are vertically shifted while retaining their shape (at the bottom). However, in other subjects the poor to moderate repeatability was the result of two completely different shaped curves (in the middle).

Our study indicates that $\mathrm{LICl}$ repeatability is subject specific and shows a high intersubject variability, just as other excitability measures (e.g. the $\mathrm{LICl}$ curve). This observed large variation may limit the applicability of TMS as a tool to monitor the disease process or to evaluate the effect of an intervention. For the interpretation of longitudinal study results, it is necessary to know the subject specific $\mathrm{LICl}$ repeatability to prevent erroneous interpretation that may result from large variability. Furthermore, studies with multiple repeated sessions and longer inter-session periods are needed to investigate the long-term repeatability and stability of $\mathrm{LICl}$. For example, Kimiskidis et al. (2004) demonstrated the stability of the corticomotor threshold on an individual and group level, using seven sessions over a period of five years.

We performed the same TMS session twice, under equal circumstances. Approximately half of the subjects showed similar LICl curves both times, while the other half had two (completely) different curves. The large variation in repeatability, might be due to a difference in coil positioning between sessions. Perhaps positioning is more critical in certain subjects because of their individual sulcus anatomy. Other explanations for a poor $\mathrm{LICl}$ repeatability could be a difference in mental state between sessions or differences in sleep pattern and/or intake of neuroactive substances (like alcohol, caffeine or nicotine) during the periods before the two sessions. Furthermore, females were measured during different phases of their menstrual cycle, as approximately one week was kept between repeated sessions. Despite inconclusive findings (Cerqueira et al., 2006; Conte et al., 2007; Hattemer et al., 2007; Huber et al., 2013; Kähkönen et al., 2003; Lang et al., 2008; Orth et al., 2005; Silvanto and Pascual-Leone, 2008; Smith et al., 1999; Ziemann et al., 1995; Zoghi et al., 2015), all these factors are potential confounders of cortical excitability that may contribute to poor repeatability. We have deliberately chosen not to compensate for these factors because, in order for TMS to become a clinical tool, relevant changes in cortical excitability should outweigh these potential confounders.

\subsubsection{Repeatability at the group level}

Repeatability was good for the averaged LICI curves: manual (overall: ICC $=0.91$ ) and robot group (overall: ICC $=0.95$ ). These findings are similar to the low inter-session $\mathrm{LICI}$ 
variability reported by Badawy et al. (2012): range rho-c: 0.93-0.95, and the high testretest reliability in the motor cortex for LICI reported by Farzan et al. (2010): Cronbach's alpha $=0.88$. Due to the averaging over subjects, it was still possible to find very high repeatability at a group level. However, repeatability decreased when individual $\mathrm{LICI}$ curves were correlated between sessions: manual (overall: ICC $=0.76$ ) and robot group (overall: ICC $=0.84$ ).

\subsubsection{Repeatability of manual and robot-guided coil positioning}

Minimal differences in LICI repeatability were found when comparing manual to robotguided coil positioning. Although repeatability was slightly higher for robot-guided positioning, also in this group poor repeatability was found for individual subjects and ISIs. Fleming et al. (2012) did not find any significant differences in SICI or ICF reliability between a hand-held and navigated figure-of-eight coil. Neuronavigation did not further improved SICI and ICF reliability (Fleming et al., 2012), just as in our study robot-guided coil positioning did not improved LICI repeatability. Hence, the added value of neuronavigation or robot-guided coil positioning in paired pulse TMS studies seems limited and unnecessary. This would make the applicability of TMS as a tool for diagnostics and/or therapy evaluation easier. However, it should be noted that subjects participated in either the manual or robot-guided coil positioning group and did not underwent both types of coil positioning. As no direct comparison was made, this study only provides indirect evidence for the limited added value of robot-guided coil positioning.

\subsubsection{Limitations}

To assess $\mathrm{LICl}$ repeatability at the subject and group level, inhibition ratios of multiple ISIs and both hemispheres were pooled. As outcomes from the dominant and nondominant hemisphere or from different ISIs are (likely) dependent within subjects, data pooling violates the ICC assumption of independency. Thus, data pooling might lead to type I errors.

To compensate for muscle pre-activation, we rejected trials containing EMG activity in the $50 \mathrm{~ms}$ preceding stimulation. We only checked for EMG activity prior to the conditioning pulse, as for ISIs 50 and $100 \mathrm{~ms}$ the period between the end of the MEP and the second test pulse was too short. If less than 25 trials remained, that specific ISI was not taken into account. Although it is unknown how many pulses are needed for $\mathrm{LICl}$ estimation, a minimum of 20 and 25 pulses is needed for reliable $\mathrm{SICI}$ and ICF estimation (Chang et al., 2016) and at least 20-30 trials for single pulse TMS (Goldsworthy et al., 2016). Overall, less than $4 \%$ of the ISIs were not taken into account, of which $60 \%$ belonged to one subject (nr. 4). We compensated for muscle 
pre-activation because, due to spinal facilitation, MEP amplitudes are larger in contracted than in relaxed muscles (Abbruzzese and Trompetto, 2002; Hess et al., 1987; Wassermann et al., 2008), resulting in an overestimation of the peak-to-peak amplitude. However, since we calculated the ratio between mean MEP amplitudes (TR/CR (\%)), EMG trial rejection might not even be necessary in case the conditioning and test response are equally affected by muscle pre-activation. Paired pulse studies are needed to investigate the influence of muscle pre-activation on inhibition ratios.

Another limitation is that robot-guided coil positioning was applied without neuronavigation. Navigation based on neuroimaging enables coil positioning based on the underlying brain anatomy by selecting the stimulation regions on the image data. However, Fleming et al. (2012) found that neuronavigation did not further improved SICI and ICF reliability.

\subsection{Conclusion}

A large variation in repeatability was found at the level of individual subjects and ISIs. While good repeatability was found for averaged $\mathrm{LICl}$ curves on a group level, it decreased when the inter-subject variability was taken into account. For the applicability of TMS as a clinical tool, the focus should move from a group level towards the level of an individual patient. For a correct interpretation of longitudinal study outcomes it is important to know the subject specific LICI repeatability and to analyze each ISI individually. The limited added value of robot-guided coil positioning in paired pulse TMS studies, makes it easier to use TMS as a tool for diagnostics and/or therapy evaluation.

\section{Acknowledgements}

The authors wish to thank Carin Eertman and Esther ter Braack for their assistance during the paired pulse TMS measurements and all the subjects for their participation.

\section{Funding}

This study was financed by the Dutch TWIN foundation for neuromodulation. The funding source played no role in the design of the study, collection, analysis and interpretation of the data, and writing of the manuscript. 



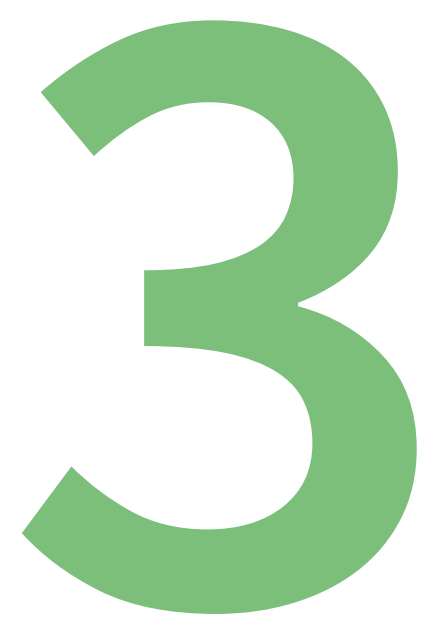

\section{Accurate coil positioning is important for single and paired pulse TMS on the subject level}

A.A. de Goede, E.M. ter Braack and M.J.A.M. van Putten Brain Topography 2018;31(6):917-930 


\section{Abstract}

Objective: Function-guided navigation is commonly used when assessing cortical excitability using transcranial magnetic stimulation (TMS). However, the required accuracy, stability and the effect of a change in coil positioning are not entirely known. This study investigates the accuracy of function-guided navigation for determining the hotspot. Furthermore, it evaluates the effect of a change in coil location on the single and paired pulse excitability measures: motor evoked potential (MEP) amplitude, TMS evoked potential (TEP) and long intracortical inhibition ( $\mathrm{LICl}$ ), and of a change in coil orientation on $\mathrm{LICl}$.

Methods: Eight healthy subjects participated in the single pulse study and ten in the paired pulse study. A robot-guided navigation system was used to ensure accurate and stable coil positioning at the motor hotspot as determined using function-guided navigation. In addition, we targeted four locations at $2 \mathrm{~mm}$ and four at $5 \mathrm{~mm}$ distance around the initially defined hotspot and we increased and decreased the coil orientation by $10^{\circ}$.

Results: In none of the subjects, the largest MEP amplitudes were evoked at the originally determined hotspot, resulting in a poor accuracy of function-guided navigation. At the group level, a change in coil location had no significant effect on the MEP amplitude, TEP or LICI and a change in coil orientation did not significantly affected LICl. However, at the subject level significant effects on MEP amplitude, TEP and $\mathrm{LICl}$ were found for changes in coil location or orientation, although absolute differences were relatively small and did not show a consistent pattern.

Conclusion: This study indicates that a high accuracy in coil positioning is especially required to measure cortical excitability reliably in individual subjects using single or paired pulse TMS. 


\subsection{Introduction}

Transcranial magnetic stimulation (TMS) is a non-invasive technique for assessing cortical excitability (Barker et al., 1985). Initially, only the integrated corticospinal excitability could be measured by combining single pulse TMS with electromyography (EMG) (Abbruzzese and Trompetto, 2002; Valls-Solé et al., 1992). However, paired pulse TMS-EMG focuses more on the excitability of cortical neurons (Abbruzzese and Trompetto, 2002; Kujirai et al., 1993), while TMS combined with electroencephalography (EEG) measures the direct neuronal response (Ilmoniemi et al., 1997; Ilmoniemi and Kičić, 2010). Although TMS is used to study a variety of neuropsychiatric conditions (Chen et al., 2008; Ni and Chen, 2015), it is only routinely used for therapeutic purposes. The applicability of TMS as a clinical tool for diagnostics or therapy evaluation is limited, mainly due to a high intra- and inter-subject variability of excitability measures (Ni and Chen, 2015; Wassermann, 2002).

Part of the intra- and inter-subject variability is caused by fluctuations in physiological processes (Goldsworthy et al., 2016; Schmidt et al., 2015). However, it is difficult to control these processes, such as the level of muscle pre-activation (Darling et al., 2006; Hess et al., 1987), the state of ongoing cortical oscillatory rhythms (Bergmann et al., 2012; Sauseng et al., 2009) and both the attention level and arousal state of participants (Mars et al., 2007). Non-biological causes of variation are easier to address. For example, the variability of excitability measures can be reduced by minimizing external noise, increasing the number of trials (Chang et al., 2016; Goldsworthy et al., 2016) and optimizing the coil positioning, in terms of location, orientation and tilt (Amassian et al., 1989a; Hess et al., 1987; Schmidt et al., 2015). Of these three suggestions, accurate and stable positioning of the coil is probably the most difficult to achieve, while its contribution to reducing variability is largely unknown (Schmidt et al., 2015).

Several navigation methods can be used to determine the coil location, while the coil is placed by default at $45^{\circ}$ from the midline (orientation) and tangentially to the stimulation target (tilt) (Groppa et al., 2012). The traditional function-guided method uses signature outputs, such as motor responses, to locate a hotspot in the primary motor cortex (Barker et al., 1985; Rossini et al., 2015). To determine the hotspot for a particular target muscle, the coil is moved gradually over the motor cortex to find the location that evokes the largest EMG responses, while applying a series of pulses at a relatively high intensity (Rossini et al., 1994, 2015). The hotspot is not only used as stimulation location in TMS-EMG studies, but is also a preferred target for TMS-EEG. When other targets are to be stimulated, such as the dorsolateral prefrontal cortex, the hotspot is first targeted to evaluate the resting motor threshold (rMT) and to determine the stimulation intensity (Kähkönen et al., 2005; Komssi et al., 2004, 2007). Therefore, 
correct coil positioning at the hotspot is important for a broad range of TMS studies.

Alternatively, neuronavigation methods make use of individual brain imaging data to position the coil above a selected cortical area (Lefaucheur, 2010; Schönfeldt-Lecuona et al., 2005; Sparing et al., 2008). It is often combined with a frameless stereotaxic system to not only ensure accurate positioning of the coil, but also coil stability throughout the TMS session (Cincotta et al., 2010; Lefaucheur, 2010; Sparing et al., 2010). Despite the high accuracy and stability of neuronavigation (Herwig et al., 2001; Lefaucheur, 2010; Sparing et al., 2010), function-guided navigation is still a commonly used method to determine the hotspot since it can be easily performed. However, little is known about the accuracy and stability required for coil positioning during both single and paired pulse TMS, and about the effect of a change in coil positioning, in terms of location, orientation and tilt.

In this study, we investigate the accuracy of function-guided navigation for determining the hotspot. We evaluate the effect of a 2 or $5 \mathrm{~mm}$ change in coil location on the MEP amplitude, TMS evoked potential (TEP) and long intracortical inhibition (LICI). In addition, we evaluate the effect of a $10^{\circ}$ change in coil orientation on $\mathrm{LICl}$. Furthermore, we investigate the stability of these single and paired pulse TMS parameters at different locations at and around the hotspot. The hotspot was determined using functionguided navigation, after which a robot-guided navigation system was used to ensure accurate and stable coil positioning during stimulation.

\subsection{Materials and methods}

Single and paired pulse TMS data was collected as part of two larger trials (trial ID: NL36317.044.11 for single pulse data and trial ID: NL49854.044.14 for paired pulse data). Both study protocols were approved by the local medical ethics committee (Medisch Spectrum Twente, Enschede, the Netherlands) and were in accordance with the Declaration of Helsinki. We followed the guidelines for the use of TMS in clinical practice and research (Rossi et al., 2009). Part of the dataset was previously used in another context by ter Braack et al. (2013b) and by de Goede and van Putten (2017).

\subsubsection{Subjects}

Healthy adults (18 years or older) were included after giving written informed consent and filling out the Screening Questionnaire before TMS (Rossi et al., 2011) and the Dutch Handedness Questionnaire (van Strien, 1992, 2003). Subjects with contraindications to TMS were excluded. Eight subjects ( 7 males, mean age $24 \pm 1.6$ years; range 23-27 years, all right-handed) were included in the single pulse TMS study and another ten subjects ( 4 males, mean age $28 \pm 8.8$ years; range $22-51$ years, 9 right-handed) in the 
paired pulse TMS study. In the single pulse TMS study, EMG and EEG data was obtained simultaneously, while in the paired pulse TMS study only EMG data was measured.

\subsubsection{Coil positioning}

Positioning of the TMS coil, with an optical tracking accuracy of $1 \mathrm{~mm}$ in every direction, was achieved using a robot-guided navigation system (Smartmove, ANT Neuro, Enschede, the Netherlands (ANT Neuro, 2018)). The position of the robot, coil and subject were continuously tracked by a Polaris infrared camera system (Northern Digital, Waterloo, Canada). Through a calibration procedure the robot, TMS coil and tracking system were registered to a common coordinate system. Subjects were tracked using a headband with four passive reflective markers. A standard $1.5 \mathrm{~T}$ magnetic resonance image was used to create a head model which was linked to the subject by collecting three landmarks and approximately 300 additional points on the scalp with a tracking pointer. We used a robotic arm holding the coil for accurate positioning at the stimulation target. Displacements from the target were automatically detected and actively corrected by the robotic arm to ensure accurate and stable coil positioning throughout the TMS session.

\section{Stimulation target: motor hotspot}

In all subjects, the left motor hotspot of the abductor digiti minimi (ADM) muscle was the primary stimulation target. The hotspot was located by manual function-guided navigation. The location in the motor cortex that evoked the largest MEPs was marked on the created head model, which was linked to the subject. Hereafter robot-guided navigation was used for stable coil positioning at the indicated hotspot. The TMS coil was placed tangentially at the ADM hotspot, with the handle pointing backwards and laterally at an angle of $45^{\circ}$ from the midline. In both the single and paired pulse TMS study, the hotspot was stimulated at the start of the study (session 1). For an overview of the stimulated targets, see Figure 3.1.

\section{Change in coil location}

In addition to the hotspot, we targeted four locations at a distance of $2 \mathrm{~mm}$ and four at a distance of $5 \mathrm{~mm}$ from the hotspot, see Figure 3.1. The coil was either moved in an anterior-medial (AM), anterior-lateral (AL), posterior-medial (PM) or posterior-lateral $(\mathrm{PL})$ direction. Except for this change in coil location, the orientation $\left(45^{\circ}\right.$ from the midline) and tilt (tangentially to the stimulation target) were kept constant.

\section{Change in coil orientation}

In the paired pulse study, we also evaluated the effect of $10^{\circ}$ change in coil orientation, see Figure 3.1. The angle from the midline was decreased to $35^{\circ}$ (session 10) and then 


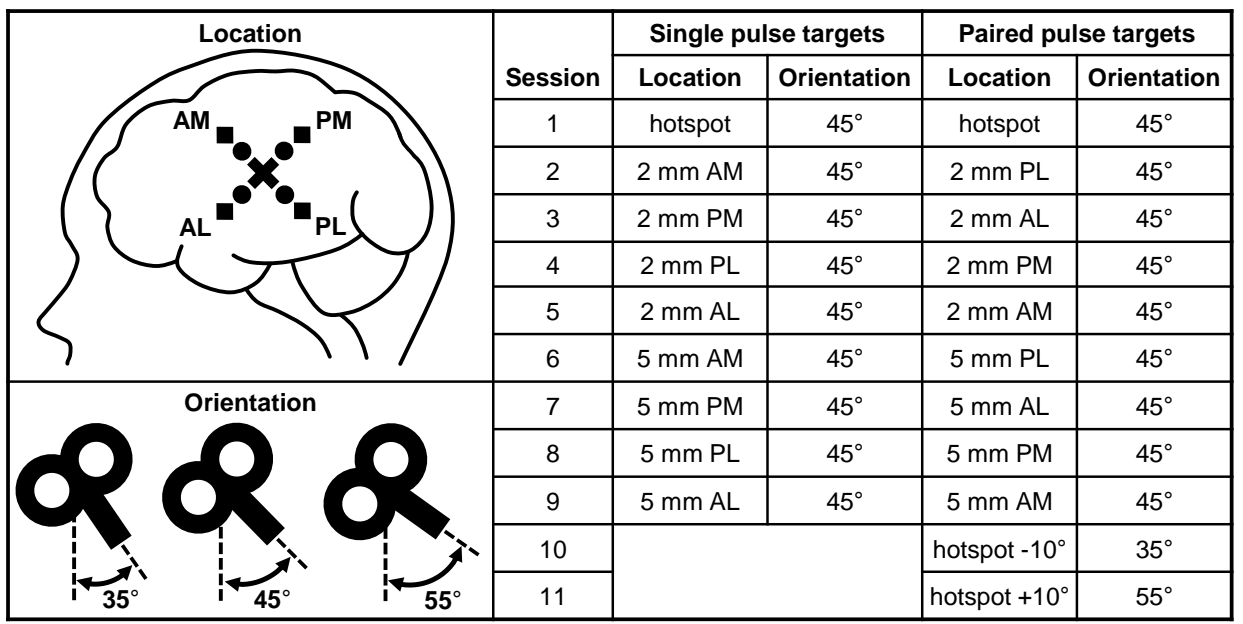

Figure 3.1: Overview of the stimulated single and paired pulse targets. The stimulation locations are represented by a cross for the hotspot, by circles for the four locations at a distance of $2 \mathrm{~mm}$ from the hotspot and by squares for the four locations at a distance of $5 \mathrm{~mm}$ from the hotspot; $\mathrm{AM}=$ anterior-medial, $\mathrm{PM}=$ posterior-medial, $\mathrm{PL}=$ posterior-lateral and $\mathrm{AL}=$ anterior-lateral.

increased to $55^{\circ}$ (session 11). We only changed the coil orientation, while maintaining the same location (at the hotspot) and tilt (tangentially to the hotspot).

\subsubsection{Stimulation protocol}

Each subject was seated comfortably in a chair, with the right hand pronated in a relaxed position. Subjects were instructed to keep their eyes open and to look straight ahead during stimulation. In addition, we applied noise masking and placed a thin layer of foam between the coil and the head of the subject in the single pulse TMS study.

Biphasic TMS pulses, with pulse duration of $400 \mu \mathrm{s}$, were applied using a figure-of-eight air film 70 mm coil and a Magstim Rapid ${ }^{2}$ Stimulator (both from The Magstim Company Ltd, Whitland, United Kingdom). The intensity of stimulation depended on the rMT, which was defined as the minimum intensity needed to evoke at least five MEPs, of at least $50 \mu \mathrm{V}$, out of ten consecutive pulses (Groppa et al., 2012; Rossini et al., 2015).

\section{Single pulse TMS protocol}

Each target was stimulated by 75 single pulses at an intensity of $110 \%$ rMT, with a random interpulse interval between 3-4 s.

\section{Paired pulse TMS protocol}

Each target was stimulated with 10 paired pulses at five randomly applied interstimulus interval (ISIs): 100, 150, 200, 250 and $300 \mathrm{~ms}$. Both the conditioning and test pulses 
were applied at an intensity of $120 \%$ rMT. A random interval between 3.5-4.5 s was kept between pairs of consecutive pulses.

\subsubsection{EMG recording and analysis}

For the EMG recordings, two surface $\mathrm{Ag} / \mathrm{AgCl}$ electrodes were placed in a belly-tendon montage over the right ADM muscle. The ground electrode was placed on the dorsal side of the hand. In the single pulse TMS study, we recorded the EMG using an additional amplifier coupled to a 64-channel EEG amplifier. In the paired pulse TMS study, the EMG was recorded using a 74-channel EEG amplifier (all from TMSi, Oldenzaal, the Netherlands). In both studies, EMG was sampled at $2048 \mathrm{~Hz}$ and lowpass filtered with an anti-aliasing filter with a cut-off frequency of $550 \mathrm{~Hz}$.

Even though subjects were asked to fully relax their right ADM muscle, EMG recordings were afterwards checked for muscle pre-activation. Trials containing EMG activity larger than $50 \mu \mathrm{V}$ in the $50 \mathrm{~ms}$ preceding a single or conditioning pulse were excluded in the single and paired pulse study, respectively.

\section{Single pulse EMG analysis: MEP amplitude}

For each subject and each target, we calculated the peak-to-peak amplitudes of the EMG responses. To perform statistical analysis at the group level, for each subject the mean MEP amplitude per target was taken.

\section{Paired pulse EMG analysis: LICI}

$\mathrm{LICI}$ was determined separately for each subject, target and ISI. First, we calculated the mean peak-to-peak amplitude of the responses to the conditioning and test pulses. Next, we calculated the ratio between this mean amplitude of the test response (TR) and this mean amplitude of the conditioning response (CR), expressed as a percentage: $100 \cdot$ TR/CR (\%) (Valls-Solé et al., 1992). This ratio represents inhibition for values below $100 \%$ and facilitation for values above $100 \%$.

\subsubsection{EEG recording and analysis}

We continuously recorded the EEG during single pulse TMS using a 64-channel EEG amplifier (TMSi, Oldenzaal, the Netherlands) and a TMS-compatible EEG cap (ANT Neuro, Enschede, the Netherlands). The ground electrode was placed between electrode positions Fz and Fpz. The EEG was sampled at $2048 \mathrm{~Hz}$ and low-pass filtered with an anti-aliasing filter with a cut-off frequency of $550 \mathrm{~Hz}$.

The EEG was analyzed in the common average montage. Trials were defined from $50 \mathrm{~ms}$ before to $350 \mathrm{~ms}$ after every TMS pulse, resulting in 75 trials of $400 \mathrm{~ms}$ for each electrode 
position. After removal of the baseline, single trial principal component analysis (PCA) was performed using 40 principal components. For a detailed description of the PCA methods, see ter Braack et al. (2013a). The first four PCA components were removed, after which trials were filtered with a fourth order Butterworth bandpass filter between 1-45 Hz. Hereafter, trials were averaged, resulting in a TEP for each electrode position.

\subsubsection{Statistical analysis}

To evaluate the accuracy of function-guided navigation for determining the hotspot, we tested whether the highest MEP amplitudes were evoked at the hotspot. For the paired pulse study, the MEP amplitudes to the 50 conditioning pulses were taken. Oneway ANOVA was used to test if there were significant differences in MEP amplitudes measured at the hotspot compared to the other stimulation targets, while Levene's test was used to assess equality of variances.

To evaluate the effect of a change in coil location at the group level, one-way repeated measures ANOVA with Greenhouse-Geisser correction was used to test if there were significant differences in mean MEP amplitudes between the nine stimulation targets. To evaluate stability, we used Levene's test to determine if there was a significant difference in mean MEP amplitude variance between targets. At the subject level, we used the outcomes of the one-way ANOVA and Levene's test, as described in the first paragraph, to determine if there were significant differences in MEP amplitudes and amplitude variance between all nine targets.

To compare the EEG responses measured at the hotspot and at the locations around the hotspot, we used the nonparametric cluster-based permutation analysis (Maris and Oostenveld, 2007) as implemented in the FieldTrip toolbox (http://www.fieldtrip toolbox.org). At the group level, dependent samples $t$-tests were used to compare the TEPs measured between two stimulation targets. Comparisons were performed for each electrode position and each time sample between 0-300 ms. Only $t$-values with a $p$-value $<0.05$ were clustered based on neighboring electrodes $(n \geq 2)$ and adjacent time samples. The summed $t$-value of each cluster was statistically compared to the distribution of clusters obtained through a permutation test. Here, all trials were randomly assigned to either of the two locations for 1500 times (Monte Carlo estimate). Clusters in the original data were considered to be significant if less than $5 \%$ of the summed $t$-values obtained by permutation were larger than the cluster value observed in the original data. Analysis at the subject level was similar to the group level statistics, except that independent samples $t$-tests were used to compare the EEG trials measured at the nine stimulation locations at and around the hotspot.

To evaluate the effect of a change in coil location and orientation at the group level, 
one-way repeated measures ANOVA with Greenhouse-Geisser correction was used to test if there were significant differences in $\mathrm{LICl}$ measured at the eleven stimulation targets. Each ISI (100, 150, 200, 250 and 300 ms) was individually tested. To evaluate stability, we used Bartlett's test to assess equality of variances. A nonparametric test was used as the data was not normally distributed. At the subject level, one-way ANOVA was used to test for significant differences between targets in $\mathrm{LICI}$ for each ISI and Bartlett's test for a significant difference in $\mathrm{LICl}$ variance. Propagation of uncertainty rules were applied to define the standard deviation (SD) belonging to a $\mathrm{LICl}$ ratio (Farrance and Frenkel, 2012).

In case significant differences were found with the single or paired pulse TMS-EMG paradigms, we further evaluated the size of these differences to get insight into the impact of changes in coil location and orientation. Only for the significant targetcomparisons we calculated the absolute difference in mean MEP amplitude or $\mathrm{LICl}$ between both targets and averaged these values.

For the statistical, as well as EMG and EEG analysis, we used Matlab (version R2015a, The Mathworks, Natick, MA, USA). For all statistical analysis $p$-values $<0.05$ were considered statistically significant and additionally adjusted for the amount of performed tests (Bonferroni corrected with $n=36$ and $n=55$ for the single and paired pulse study, respectively). For the ANOVA's, post hoc tests were performed to identify which targets differ from each other. Effect sizes were calculated using the partial etasquared, defined as

$$
\eta_{p}^{2}=\frac{S S_{\text {effect }}}{S S_{\text {effect }}+S S_{\text {error }}}
$$

\subsection{Results}

In one subject (paired pulse $\mathrm{nr} .10$ ) the locations at $5 \mathrm{~mm}$ distance were skipped to shorten the measurement time because of discomfort and in one subject (paired pulse nr. 8) coil orientation was not changed due to technical problems with the navigation system. All other participants tolerated the stimulation protocol well.

\subsubsection{Hotspot accuracy}

In all subjects, the largest MEP amplitudes were not evoked at the hotspot. In five out of eight subjects from the single pulse study and all ten subjects from the paired pulse study, amplitudes were significantly higher at another location compared to the hotspot, see filled dots in Figure 3.2. In the single pulse study, five out of eight subjects showed inequality of variances, being significantly lower at the hotspot in only one. In seven out of ten subjects from the paired pulse study, a significantly lower variance was measured at the hotspot, see solid lines in Figure 3.2. 

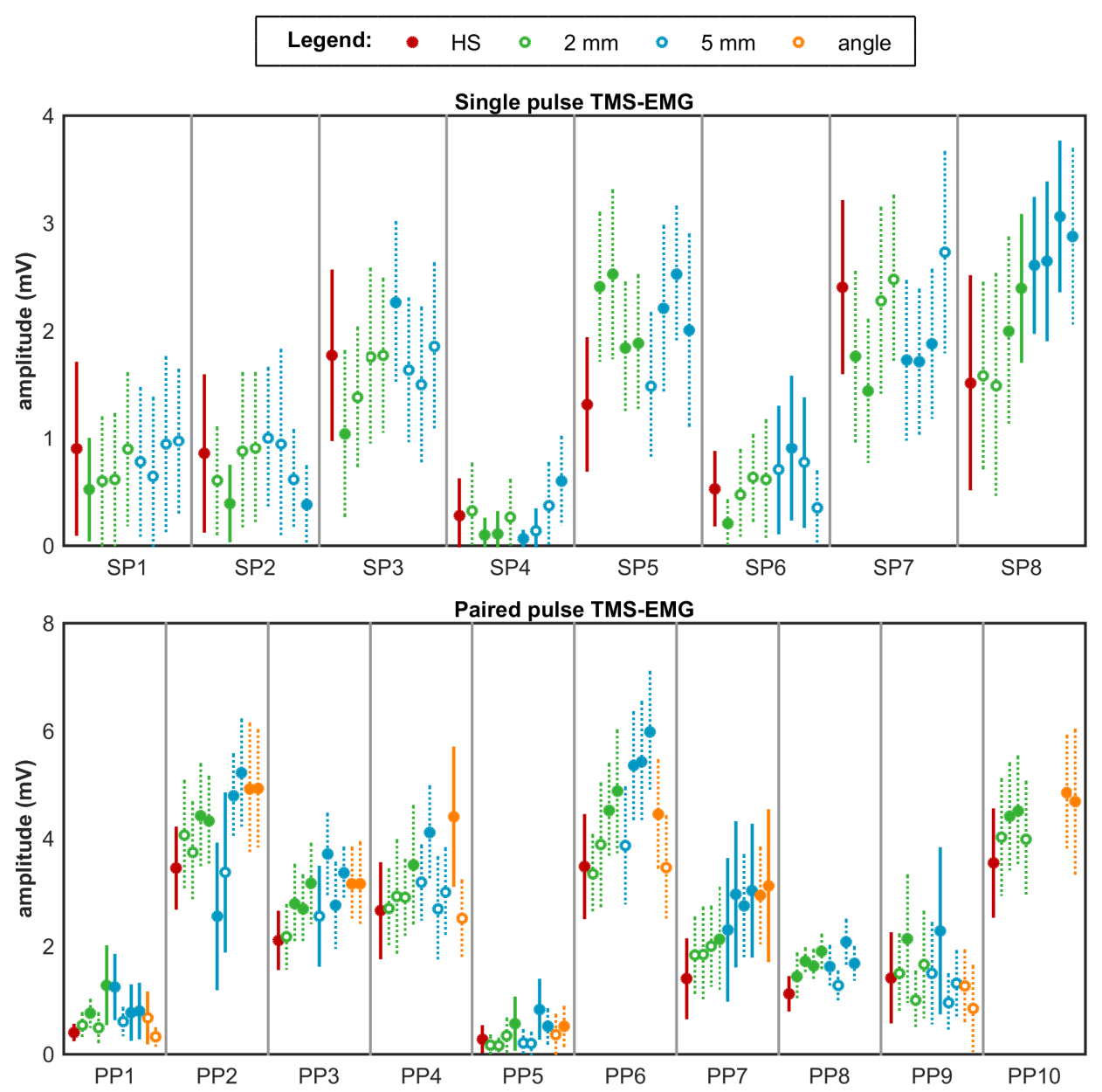

Figure 3.2: Outcomes of hotspot accuracy. The top row shows per subject the mean \pm SD of the MEP amplitudes per target in the single pulse study. The bottom row shows per subject the mean \pm SD of the conditioning MEP amplitudes per target in the paired pulse study. In red, the outcomes measured at the hotspot (HS); in green, at a distance of $2 \mathrm{~mm}$ from the hotspot; in blue, at a distance of $5 \mathrm{~mm}$ from the hotspot; and in orange, for a $10^{\circ}$ change in coil orientation. Targets that differ significantly from the hotspot in mean MEP amplitude and amplitude variance are indicated with filled dots and solid lines, respectively. 


\subsubsection{Single pulse TMS-EMG outcome: MEP amplitude}

At the group level, no significant differences were found for the mean MEP amplitudes measured at the nine stimulation targets $\left(F(3.2,22.3)=1.7, p=0.20, \eta_{p}^{2}=0.19\right)$, nor for the amplitude variances $(F(8,63)=0.5, p=0.86)$, see top row Figure 3.3. For the MEP amplitudes (mean \pm SD) measured at each target, see Table 2 of the Supplementary material.

At the subject level, significant differences were found in all eight subjects (range 624 out of 36 possible comparisons) and for all nine targets (range 25-39 out of 64 possible comparisons), see light red bars in Figure 3.4 and Table 2 of the Supplementary material. Three out of eight subjects showed equality of variances (SP3: $F(8,655)=1.0$, $p=0.42$; SP5: $F(8,661)=1.3, p=0.25$; SP7: $F(8,641)=0.8, p=0.61)$. Between subjects the absolute difference in mean MEP amplitude measured at two significant targets varied from $0.3-1.0 \mathrm{mV}$, while between targets the absolute difference for significant subjects in mean MEP amplitude measured at two significant targets varied from 0.5$0.7 \mathrm{mV}$, see red bars in Figure 6 of the Supplementary material. No clear differences were seen between the four locations at $2 \mathrm{~mm}$ distance and the four locations at $5 \mathrm{~mm}$ distance from the hotspot, nor between the medial and lateral targets, see dark red bars in Figure 3.4. For two subjects the MEP amplitude at each of the nine stimulation targets is shown in the top row of Figure 3.5.

\subsubsection{Single pulse TMS-EEG outcome: TEP}

At the group level, we found no significant effect of a change in coil location on the TEPs measured at the hotspot compared to targets surrounding the hotspot using cluster-based permutation analysis (no significant clusters), see middle row Figure 3.3. Only one significant cluster $(p<0.001$; parieto-occipital in the ipsilateral hemisphere between 40-90 ms) was found when comparing the TEPs measured at $2 \mathrm{~mm}$ PM and 5 $\mathrm{mm}$ PL.

At the subject level, significant clusters were found in all eight subjects (range 1026 out of 36 possible comparisons) and for all nine targets (range 19-41 out of 64 possible comparisons), see light green bars in Figure 3.4. No clear differences were seen between the four locations at $2 \mathrm{~mm}$ distance and the four locations at $5 \mathrm{~mm}$ distance from the hotspot, nor between the medial and lateral targets, see dark green bars in Figure 3.4. Although significant clusters varied over subjects and targets, most clusters were found between 105-180 ms and involved electrodes in central and parietal brain areas. For a complete overview of the cluster characteristics, see Table 3.1. For two subjects the TEP at each of the nine stimulation targets is shown in the middle row of Figure 3.5. 

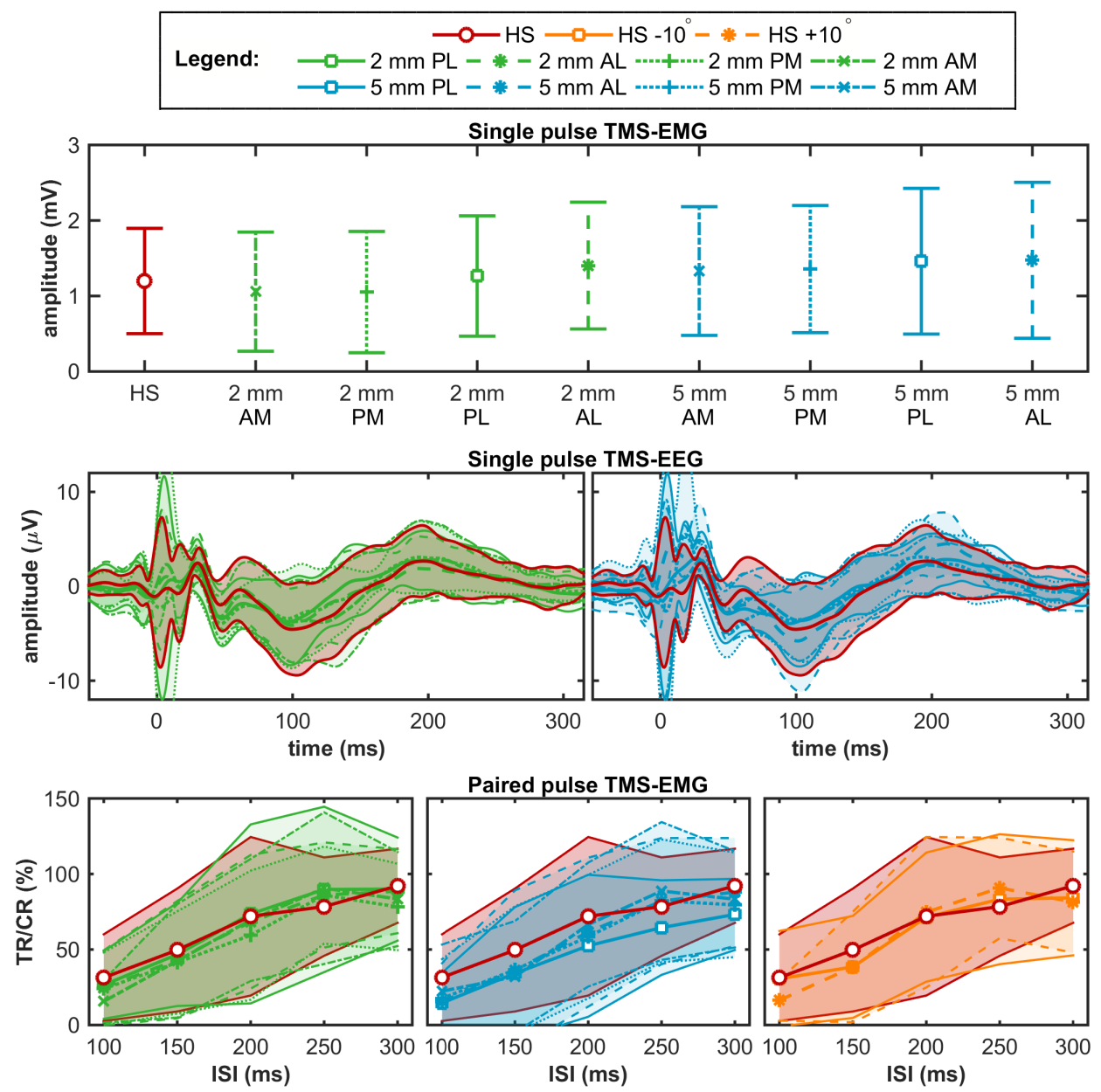

Figure 3.3: Outcomes at the group level. The top row shows per target the mean \pm SD of the mean MEP amplitude per subject in the single pulse TMS-EMG study. The middle row shows per target the mean \pm SD of the TEP at electrode position $\mathrm{Cz}$ per subject in the single pulse TMS-EEG study. The bottom row shows per target the mean \pm SD of the LICI per subject in the paired pulse TMS-EMG study. In red, the outcomes measured at the hotspot (HS); in green, at a distance of $2 \mathrm{~mm}$ from the hotspot; in blue, at a distance of $5 \mathrm{~mm}$ from the hotspot; and in orange, for a $10^{\circ}$ change in coil orientation. $\mathrm{AM}=$ anterior-medial, $\mathrm{PM}=$ posterior-medial, $\mathrm{PL}=$ posterior-lateral, $\mathrm{AL}=$ anterior-lateral, $\mathrm{TR}=$ test response, $\mathrm{CR}=$ conditioning response and $\mathrm{ISI}=$ interstimulus interval. 

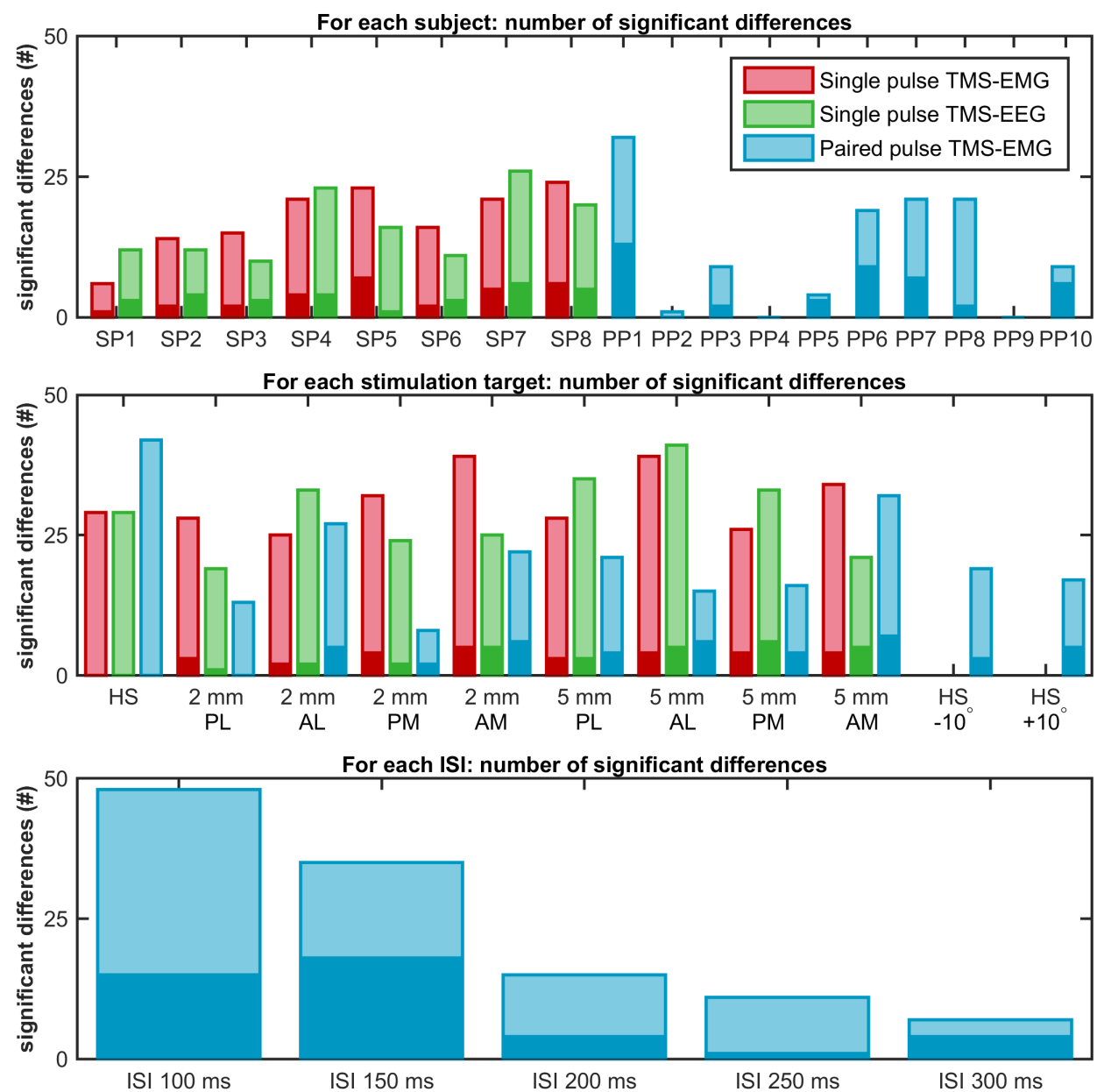

Figure 3.4: Outcomes at the subject level. Bar plots showing the number of significant differences found in each subject (top row), at each stimulation target (middle row) and for each ISI (bottom row). In red, results of the single pulse TMS-EMG study; in green, of the single pulse TMS-EEG study; and in blue, of the paired pulse TMS-EMG study. All significant differences combined are indicated by the light color bar, while differences for only the hotspot versus any other target are indicated by the dark color bar. $\mathrm{SP}=$ single pulse, $\mathrm{PP}=$ paired pulse, $\mathrm{HS}=$ hotspot, $\mathrm{PL}=$ posterior-lateral, $\mathrm{AL}=$ anterior-lateral, $\mathrm{PM}=$ posterior-medial, $\mathrm{AM}=$ anterior-medial and ISI = interstimulus interval. 

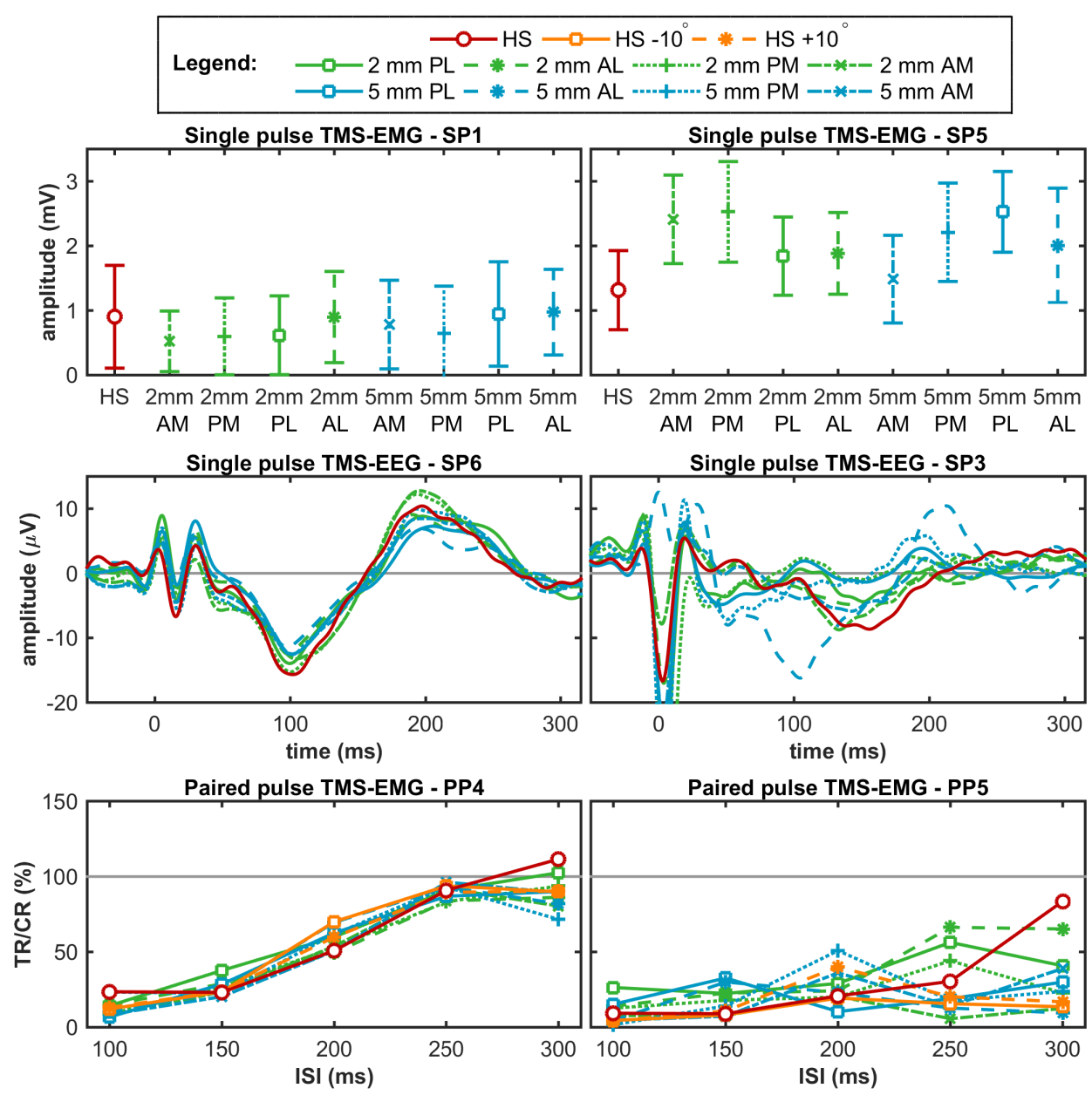

Paired pulse TMS-EMG - PP5

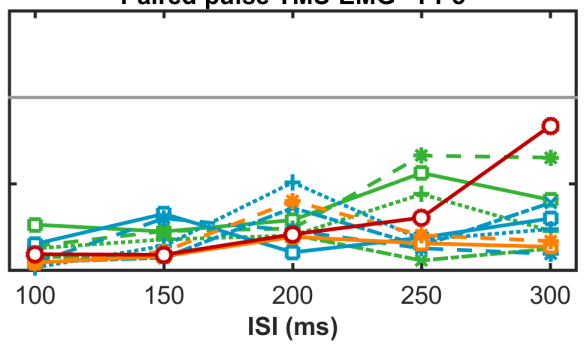

Figure 3.5: Examples of good (on the left) and poor (on the right) outcomes at the subject level. The top row shows two examples of the single pulse TMS-EMG study (mean \pm SD MEP amplitude); the middle row of the single pulse TMS-EEG study (TEP at electrode position $\mathrm{Cz}$ ); and the bottom row of the paired pulse TMS-EMG study (LICl curve). In red, outcomes measured at the hotspot (HS); in green, at a distance of $2 \mathrm{~mm}$ from the hotspot; in blue, at a distance of $5 \mathrm{~mm}$ from the hotspot; and in orange, for a $10^{\circ}$ change in coil orientation. $\mathrm{SP}=$ single pulse, $\mathrm{PP}=$ paired pulse, $\mathrm{AM}=$ anterior-medial, $\mathrm{PM}=$ posterior-medial, $\mathrm{PL}$ = posterior-lateral, $\mathrm{AL}=$ anterior-lateral, $\mathrm{TR}=$ test response, $\mathrm{CR}=$ conditioning response and $\mathrm{ISI}=$ interstimulus interval. 
Table 3.1: Overview of the characteristics of the significant clusters found in the single pulse TMS-EEG study at the subject level.

\begin{tabular}{|c|c|}
\hline Cluster characteristics & Mean ( \pm SD) \\
\hline Electrodes involved (\#) & $20 \pm 8$ \\
\hline Length (ms) & $73 \pm 31$ \\
\hline Start time (ms) & $105 \pm 72$ \\
\hline \multicolumn{2}{|l|}{ Hemisphere involved } \\
\hline Left (\%) & 26 \\
\hline Right (\%) & 10 \\
\hline Both (\%) & 65 \\
\hline \multicolumn{2}{|l|}{ Brain areas involved } \\
\hline Frontal (\%) & 69 \\
\hline Central (\%) & 99 \\
\hline Parietal (\%) & 90 \\
\hline Temporal (\%) & 51 \\
\hline Occipital (\%) & 72 \\
\hline
\end{tabular}

\subsubsection{Paired pulse TMS-EMG outcome: LICI}

At the group level, no significant differences were found for $\mathrm{LICl}$ measured at any of the eleven stimulation targets (ISI $100 \mathrm{~ms}: F(3.8,26.4)=2.0, p=0.13, \eta_{p}^{2}=0.22$; ISI $150 \mathrm{~ms}$ : $F(2.5,17.7)=1.2, p=0.34, \eta_{p}^{2}=0.14$; ISI $200 \mathrm{~ms}: F(2.7,19.3)=0.8, p=0.48, \eta_{p}^{2}=0.11$; ISI $250 \mathrm{~ms}: F(3.1,21.9)=1.2, p=0.32, \eta_{p}^{2}=0.15$; ISI $300 \mathrm{~ms}: F(3.5,24.5)=0.9, p=0.47, \eta_{p}^{2}=$ 0.11 ), nor for the variance in LICI (ISI 100 ms: $p=0.35$; ISI 150 ms: $p=0.98$; ISI 200 ms: $p$ $=0.99$; ISI 250 ms: $p=0.78$; ISI 300 ms: $p=0.96$ ), see bottom row Figure 3.3 and Tables 3-7 of the Supplementary material.

At the subject level, significant differences were found in eight out of ten subjects (range 1-32 out of 275 possible comparisons), for all eleven stimulation targets (range 8-42 out of 500 possible comparisons) and for all five ISIs (range 7-48 out of 550 possible comparisons), see light blue bars in Figure 3.4 and Tables 3-7 of the Supplementary material. None of the ten subjects showed equality of variances for all five ISIs. Between subjects the absolute difference in $\mathrm{LICl}$ measured at two significant targets varied from 15-65\%, while between targets the absolute difference for significant subjects in $\mathrm{LICl}$ measured at two significant targets varied from $26-48 \%$ and between ISIs the absolute difference for significant subjects in $\mathrm{LICI}$ measured at two significant targets varied from $30-55 \%$, see blue bars in Figure 6 of the Supplementary material. No clear differences were seen between the four locations at $2 \mathrm{~mm}$ distance and the four at $5 \mathrm{~mm}$ distance from the hotspot, nor between the medial and lateral targets, 
nor between an increase and decrease in coil angle, nor between ISIs, see dark blue bars in Figure 3.4. For two subjects, the $\mathrm{LICl}$ curves measured at each of the eleven stimulation targets are shown in the bottom row of Figure 3.5.

\subsection{Discussion}

In this study, we investigated the accuracy of function-guided navigation for determining the hotspot by evaluating the effect of small changes in coil position on single and paired pulse excitability measures. For single pulse TMS, a change in coil location did not significantly affect the mean MEP amplitudes and TEPs at the group level. Only one significant cluster was found when comparing the TEPs measured at $2 \mathrm{~mm} P M$ and $5 \mathrm{~mm}$ PL. However, at the subject level both the MEP amplitudes and EEG responses were affected. Significant differences were found in all subjects and for all targets, while the degree of change ( 2 or $5 \mathrm{~mm}$ ) and the direction of change (medial or lateral) made no difference. Similarly, for paired pulse TMS-EMG a change in coil location or orientation did not affect $\mathrm{LICl}$ at the group level, but at the subject level significant differences were found in eight out of ten subjects, for all targets and for all ISIs.

\subsubsection{Accuracy of hotspot determination}

In none of the subjects, the largest MEP amplitudes were evoked at the hotspot. When combining both studies, significant higher amplitudes were found at a different location in 15 out of 18 subjects. We determined the hotspot by function-guided navigation, so based on visual analysis of the MEP amplitude while stimulating the presumed hotspot area. Hereafter, robot-guided coil positioning was used to stimulate targets at $2 \mathrm{~mm}$ and $5 \mathrm{~mm}$ surrounding the hotspot, thereby ensuring accurate and stable positioning of the TMS coil at all of these targets.

To our knowledge the accuracy of function-guided navigation has not been studied before. A previous study by Komssi et al. (2002) stimulated four targets at $1 \mathrm{~cm}$ surrounding the hotspot, which was determined using function-guided navigation. However, they evaluated the TEP responses and did not report on the MEP amplitudes measured at the surrounding targets (Komssi et al., 2002). When comparing function-guided navigation to neuronavigation, the latter usually results in MEPs with significantly higher amplitudes (Gugino et al., 2001; Julkunen et al., 2009; Jung et al., 2010; Sparing et al., 2008). Neuronavigation uses brain imaging data to position the coil above the selected hotspot by considering inter-individual anatomical variability, instead of visual analysis of the MEP amplitude in the motor area. The fact that we were apparently not always able to determine the exact hotspot with function-guided navigation, may have resulted from the limited number of TMS pulses applied during 
the procedure and the normal variation in MEP amplitude, which is known to be large (Goldsworthy et al., 2016; Wassermann, 2002).

\subsubsection{Effect of a change in coil positioning}

We showed that small changes in coil location or orientation can result in significantly different MEP amplitudes, LICI curves or TEPs at the subject level. However, no significant differences in mean values or variances were found at the group level. In general, the fact that no significant differences were found at the group level might be explained by our small sample size. However, others found significant differences for small groups ( $n=6-8$ ) (Julkunen et al., 2009; Jung et al., 2010; Komssi et al., 2002). Although previous studies reported significantly smaller MEP and TEP amplitudes at the group level for changes in coil position (Casarotto et al., 2010; Julkunen et al., 2009; Jung et al., 2010; Komssi et al., 2002; Sparing et al., 2008), their variations in coil location $(>1 \mathrm{~cm})$ and orientation $\left(>45^{\circ}\right)$ were much larger than in our study ( 2 or $5 \mathrm{~mm}$ and $10^{\circ}$, respectively). At the group level, function-guided navigation in combination with manual positioning and holding of the coil during stimulation seems to be sufficient accurate, if the changes in location and orientation remain respectively less than $10 \mathrm{~mm}$ and $20^{\circ}$. In clinical practice, this is feasible for experienced investigators. Julkunen et al. (2009) found that when no external coil fixation is used during stimulation, coil movement is on average less than $10 \mathrm{~mm}$ from the mapped target among 20 consecutive TMS pulses (interpulse interval 4-6 s).

Most TMS studies report findings on a group level, either comparing patients to controls or pre- to post-intervention TMS sessions. Our findings indicate that in such studies function-guided navigation can be combined with positioning and holding of the coil by hand or using a stand during stimulation, even though the accuracy and stability is lower than using frameless stereotaxic neuronavigation. The most important factor to take into account when deciding which type of navigation to use is the expected difference in excitability between conditions. For example, Figure 6 of the Supplementary material shows a maximal increase or decrease in $\mathrm{LICl}$ of around $65 \%$ due to a change in coil positioning. However, differences between patients with epilepsy and healthy controls at ISI $250 \mathrm{~ms}$ have been reported to be three times as large: inhibition in controls (mean \pm SD: $68.3 \pm 37.0 \%$ ), but facilitation in patients (generalized epilepsy: $273.0 \pm 106.2 \%$ and focal epilepsy (ipsilateral hemisphere): 244.0 $\pm 76.4 \%$ ) (Badawy et al., 2007, 2014b, 2017; de Goede et al., 2016). Furthermore, in successfully treated patients facilitation may normalize to inhibition over time, while $\mathrm{LICl}$ remains increased in refractory patients (Badawy et al., 2013a). Since the effects of changes in coil positioning on $\mathrm{LICl}$ are so much smaller than the expected $\mathrm{LICl}$ differences between the two test conditions, neuronavigation methods seem to be 
superfluous in epilepsy studies.

Our results also indicate that at the subject level it is preferred to use a coil positioning method with a high accuracy and stability, such as frameless stereotaxic neuronavigation. Again, the required accuracy in coil positioning depends on the disease and study design. For clinical applications decisions have to be made based on single subject data, comparing it to either reference values for diagnostic purposes or to earlier measurements obtained in the same subject for follow-up or treatment evaluation purposes. Ultimately, TMS can only be used as a clinical tool if the differences in excitability between two conditions are larger than the intra-subject variability. In most subjects we found significant differences in measured variance, were the lowest variance was usually not measured at the hotspot determined by function-guided navigation. Although obtaining more stable MEPs using neuronavigation, the intraand inter-subject variability remains high (Gugino et al., 2001; Julkunen et al., 2009; Jung et al., 2010).

The presence of significant differences varied over subjects and targets, but we could not find a pattern in the effects of a change in coil location or orientation. In some subjects, a change in coil positioning led to more significant differences than in others, indicating large inter-subject differences in the response to TMS. For the TMS-EMG paradigm this could be related to variations in individual sulcus anatomy (Balslev et al., 2007; Kallioniemi et al., 2015). For TMS-EEG, however, it is unclear whether sulcus anatomy would have such a large impact on the TEP, as it shows similar components when stimulating the motor or premotor cortex (Ferrarelli et al., 2008; Massimini, 2005). Thus, the TEP seems to be a generic response that does not depend that much on the exact stimulation location. The fact that we did find significant differences in single subjects, but not on the group level might also be explained by the applied statistical analysis method. In TMS studies cluster-based permutation analysis is mostly used on the group level and it may not be the most optimal method for single trial comparisons, although it is designed for single subject analysis (Maris and Oostenveld, 2007). Related to this, the high sensitivity of the analysis method may explain the large variety in significant clusters that we found, both in size, length as well as location.

Although the presence of significant differences varied over targets, no clear differences were seen between the four locations at $2 \mathrm{~mm}$ distance and the four locations at $5 \mathrm{~mm}$ distance from the hotspot, nor between medial and lateral targets, nor between an increase and decrease in coil angle orientation. These findings suggest that the direction of change in location makes no difference, while Komssi et al. (2002) especially found attenuation of the TEP towards more medial stimulation sites. However, our changes in location were smaller and we only evaluated absence or presence of a significant difference. 


\subsubsection{Limitations}

This study is limited by the fact that our robot-guided navigation system only allowed adjustment of the coil location and orientation, but not the tilt. Thus, we could not evaluate the effect of a change in tilt on single and paired pulse excitability measures. Nevertheless, a recent study showed that excitability measures are mainly influenced by variations in coil location (36\%) and not so much by tilt $(5 \%)$ or orientation $(<1 \%)$ (Schmidt et al., 2015). However, in TMS-EEG studies varying the tilt might be of importance. When targets close to muscles are stimulated, associated muscle artifacts can be effectively reduced by rotating or tilting the coil wings away from the temporal muscle, while preserving the brain responses (Mutanen et al., 2013).

Even though the measurement protocol could have been optimized by randomizing the order of stimulation targets, our fixed sequence did probably not affect the outcomes. While at the subject level we found significant differences for all targets, clear differences between targets stimulated at the beginning or end of the study that may occur due to a gradual change in attention were not observed.

Furthermore, to limit the total measurement time, we applied 10 paired pulses at each ISI. Although it is unknown how many pulses are needed for $\mathrm{LICl}$ estimation, a minimum of 20 and 25 pulses is needed for reliable estimation of short intracortical inhibition and intracortical facilitation (Chang et al., 2016) and at least 20-30 trials for single pulse TMS (Goldsworthy et al., 2016). The large number of 75 single pulses was needed for reliable estimation of the TEP. In contrast to Pellicciari et al. (2016), we found no cumulative effects for MEP amplitude over time, indicated by a coefficient of determination $R^{2}$ always smaller than 0.5 , that could have influenced our findings.

\subsection{Conclusion}

In conclusion, the accuracy of function-guided navigation for determining the hotspot is poor. In none of the subjects, the largest MEP amplitudes were evoked at the presumed hotspot. At the group level, a change in coil location had no significant effect on the mean MEP amplitude, LICI and TEP or on the corresponding variances. In addition, a change in coil orientation did not significantly affect $\mathrm{LICl}$ using paired pulse TMS. At the subject level, significant differences in mean values and variances were found for both single and paired pulse TMS excitability measures, although absolute differences in MEP amplitude and $\mathrm{LICl}$ were relatively small. These findings indicate that a high accuracy in coil positioning is especially required to measure cortical excitability reliably over time in individual subjects using single or paired pulse TMS. 


\section{Acknowledgements}

The authors wish to thank Carin Eertman for her assistance during the paired pulse TMS measurements and all the subjects for their participation.

\section{Funding}

This study was financed by the Dutch TWIN foundation for neuromodulation and by the Dutch PIDON Grant, in which ANT Neuro participated as an industrial partner. The funding sources played no role in the design of the study, collection, analysis and interpretation of the data, and writing of the manuscript.

\section{Supplementary material}

See online version of this article at https://doi.org/10.1007/s10548-018-0655-6 or scan the QR code.

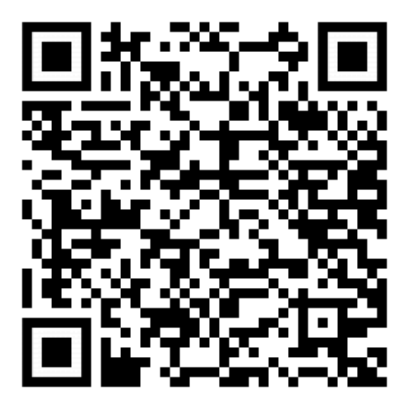






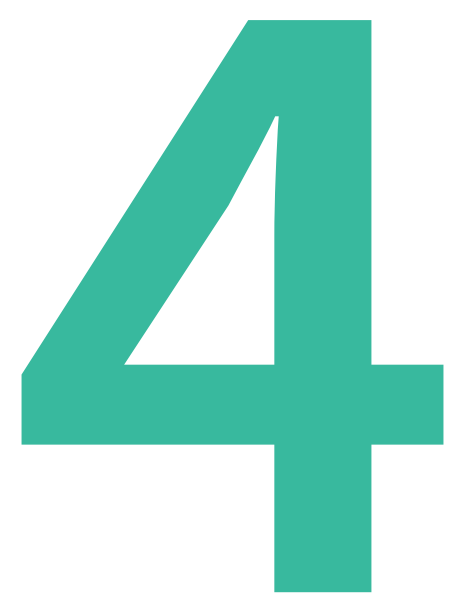

\section{Spatiotemporal dynamics of single and paired pulse} TMS-EEG responses

A.A. de Goede, I. Cumplido Mayoral and M.J.A.M. van Putten Submitted in revised form: Frontiers in Neuroscience 


\section{Abstract}

Objective: For physiological brain function a balance between excitation and inhibition is essential. Transcranial magnetic stimulation (TMS) is a technique to measure cortical excitability, while paired pulse stimulation provides additional information from inhibitory and excitatory networks. Combining TMS with electroencephalography (EEG) enables assessment of the spatiotemporal dynamics of neuronal responses in the stimulated brain. In this study, we aim to evaluate the spatiotemporal dynamics and stability of single and paired pulse TMS-EEG responses. In addition, we assess long intracortical inhibition (LICI) at the cortical level.

Methods: We studied twenty-five healthy subjects twice, with approximately one week in between. Manual coil positioning was applied in sixteen subjects and robot-guided positioning in nine. We stimulated both motor cortices with 50 single pulses and 50 paired pulses at each of the five interstimulus intervals (ISIs): 100, 150, 200, 250 and $300 \mathrm{~ms}$. To assess $\mathrm{LICl}$, the single and paired pulse responses were statistically compared using a cluster-based permutation analysis. Similarly, we compared single and paired pulse TMS-EEG responses between stimulated hemispheres, sessions and coil positioning methods to evaluate stability.

Results: We found a similar topographical distribution of the characteristic TMS-EEG components for single and paired pulse TMS. Stimulation of the dominant and nondominant hemisphere resulted in a mirrored spatiotemporal dynamics. No significant effect on the TMS-EEG responses was found for either stimulated hemisphere, time or coil positioning method. These findings demonstrate the stability and robustness of both single and paired pulse TMS-EEG responses as a potential clinical biomarker. For all five ISIs, $\mathrm{LICl}$ was characterized by significant suppression of the late $\mathrm{N100}$ and P180 components in the central areas, without affecting the early P30, N45 and P60 components. Between intervals, no clear differences were seen in $\mathrm{LICl}$ expression, indicating an overarching inhibitory mechanism regardless of ISI.

Conclusion: These spatiotemporal dynamics in healthy subjects can serve as reference values for future neuropsychiatric and pharmacological studies. 


\subsection{Introduction}

A balance between excitation and inhibition is essential for normal physiological brain function. Various neuropsychiatric conditions, such as epilepsy, autism and schizophrenia, appear to be related to an imbalance in cortical excitability (Bauer et al., 2014; Bolden et al., 2017; Bunse et al., 2014; Oliveira et al., 2018). Paired pulse transcranial magnetic stimulation (TMS) can be used to assess cortical excitability, as well as to obtain information about the relative contribution of excitatory and inhibitory networks. In the paired pulse paradigm, the interval between the conditioning and test pulse determines whether the conditioning pulse enhances or attenuates the evoked test response in the target muscle compared to an unconditioned muscle response (Kujirai et al., 1993; Rossini et al., 2015; Valls-Solé et al., 1992; Ziemann, 2017). For intervals between $50-400 \mathrm{~ms}$, the test response is normally reduced. This phenomenon is known as long intracortical inhibition (LICI) (Valls-Solé et al., 1992) and is associated with gamma-aminobutyric acid (GABA)-B receptor mediated inhibition (McDonnell et al., 2006; Müller-Dahlhaus et al., 2008).

Combining TMS with electroencephalography (EEG) makes it possible to directly investigate $\mathrm{LICl}$ at the cortical level (Daskalakis et al., 2008; Fitzgerald et al., 2008). Applying a conditioning pulse $100 \mathrm{~ms}$ in advance induces significant suppression of the average TMS evoked potential (TEP), similar to the attenuation of the muscle response (Daskalakis et al., 2008; Farzan et al., 2010; Fitzgerald et al., 2008, 2009). More recent studies found a significant decrease of all characteristic TEP components compared to the single pulse TEP (Opie et al., 2017, 2018; Premoli et al., 2014b), except for an increase of the P70 (Premoli et al., 2014b). The interstimulus interval (ISI) of 100 $\mathrm{ms}$ is most commonly used, because it results in strong $\mathrm{LICl}$ of the muscle response (Daskalakis et al., 2008; Opie et al., 2017). Only one group additionally evaluated an ISI of $150 \mathrm{~ms}$ and found a decrease of the N40, N100 and P180, but no significant effect for the P30 component (Opie et al., 2017, 2018).

The fact that significant inhibition of the P30 component was only found for ISI 100 ms and not for ISI 150 ms (Opie et al., 2017, 2018), might indicate that the relative contribution of inhibitory mechanisms associated with $\mathrm{LICl}$ varies between ISIs. Opie et al. (2017) speculated that LICI at ISI 100 ms might reflect the activation of both preand post-synaptic GABA-B receptors, whereas only pre-synaptic GABA-B receptors are activated at ISI $150 \mathrm{~ms}$. However, the same study found no significant differences between both intervals for any of the paired pulse TEP components, suggesting a uniform inhibitory effect irrespective of ISI (Opie et al., 2017). This is supported by the finding of a similar TEP modulation for short intracortical inhibition ( $\mathrm{SICl}$ ) and $\mathrm{LICl}$ (Premoli et al., 2018). To increase our understanding of the underlying inhibitory 
mechanisms, a range of intervals should be evaluated.

Besides the possibility of measuring $\mathrm{LICl}$ at the cortical level, TMS-EEG has a good spatial and temporal resolution (Fecchio et al., 2017; Ilmoniemi et al., 1997). Applying a single pulse to the motor cortex results in an immediate, localized and strong response at the stimulation site. Within 5-10 ms activation spreads to adjacent ipsilateral motor and premotor areas, followed by the activation of contralateral homologous cortical areas after 20 ms (Ilmoniemi et al., 1997; Komssi et al., 2002). Based on topographical plots, the P30 component is located in central areas, the N45 in contralateral frontal areas, the P70 over the stimulation site, where the N100 and P180 components show a wide distribution over central and centrofrontal areas, respectively (Bonato et al., 2006; Ferreri et al., 2011; Paus et al., 2001; Premoli et al., 2014a). For ISIs 100 and 150 ms, the paired pulse TEP has a similar spatiotemporal dynamics and $\mathrm{LICl}$ is expressed at the location of the corresponding TEP component (Opie et al., 2017, 2018; Premoli et al., 2014b).

So far, our knowledge of the paired pulse TEP and $\mathrm{LICl}$ is solely based on measurements performed at ISIs 100 and $150 \mathrm{~ms}$. In this study we use five ISIs between 100-300 ms to stimulate both hemispheres in healthy subjects during two sessions one week apart. This study aims to evaluate the spatiotemporal dynamics and stability of the healthy single and paired pulse TEP. Furthermore, we aim to assess $\mathrm{LICl}$ at the cortical level.

\subsection{Materials and methods}

The study protocol (trial ID: NL49854.044.14) was in accordance with the Declaration of Helsinki and approved by our local medical ethics committee (Medisch Spectrum Twente, Enschede, the Netherlands). Furthermore, we followed the guidelines for the use of TMS in clinical practice and research (Rossi et al., 2009). This study focused on the TMS-EEG data, while we have previously described the outcomes of TMS combined with electromyography (EMG) obtained from the same dataset (de Goede and van Putten, 2017; de Goede et al., 2018).

\subsubsection{Subjects}

Healthy adults (18 years or older) were included after giving written informed consent. Subjects with contraindications to TMS as described in the Screening Questionnaire before TMS (Rossi et al., 2011) were excluded. Twenty-five subjects ( 5 males, mean age $28.2 \pm 8.3$ years; range $20-51$ years, 23 right-handed) were included in this study. Of the originally thirty-four inclusions, data of nine subjects were excluded from analysis: one did not complete the first session after not feeling well, one could not be measured a second time within 1-2 weeks due to illness, two could not be stimulated at $120 \%$ 
resting motor threshold (rMT) due to a high rMT and in five it was not possible to stimulate both hemispheres during both sessions due to technical problems or a high rMT.

To determine handedness subjects filled out the Dutch Handedness Questionnaire (van Strien, 1992, 2003).

\subsubsection{TMS protocol}

Subject were instructed to keep their eyes open, their head in a fixed position and both hands pronated and relaxed during stimulation. To mask the sound of the TMS pulses, each subject listened to adaptive noise played at a maximum intensity of $95 \mathrm{~dB}$ (ter Braack et al., 2013b).

Both motor hotspots of the abductor digiti minimi (ADM) muscle were stimulated using biphasic TMS pulses, with a pulse duration of $400 \mu \mathrm{s}$, applied by a Magstim Rapid ${ }^{2}$ stimulator (The Magstim Company Ltd, Whitland, United Kingdom). Stimulation intensity depended on the rMT, which was defined as the minimum intensity needed to induce at least five motor evoked potentials (MEPs), with an amplitude larger than 50 $\mu \mathrm{V}$, out of ten consecutive pulses. First 50 single pulses were applied, followed by 50 paired pulses at each of the five randomly applied ISIs: 100, 150, 200, 250 and $300 \mathrm{~ms}$. The single pulse intensity, as well as the conditioning and test pulse intensities, were set to $120 \%$ rMT. A random interval ranging from 3.5-4.5 s was kept between single pulses and pairs of paired pulses.

All subjects underwent this TMS session twice under equal circumstances, including the same measurement set-up, coil positioning method, investigators and time of the day. Both sessions took place approximately one week apart (mean 7.4 days; range 6-15 days).

\subsubsection{Coil positioning}

The $70 \mathrm{~mm}$ figure-of-eight air film coil (The Magstim Company Ltd, Whitland, United Kingdom) was placed tangentially at the ADM hotspot, with the handle pointing backwards and laterally at a $45^{\circ}$ angle with the midline. In sixteen subjects the coil was positioned and held in place manually by the same investigator, during both TMS sessions. In the other nine subjects, a robot-guided system (Smartmove, ANT Neuro, Enschede, the Netherlands) was used for coil positioning. The position of the subject with respect to the coil was continuously monitored by a Polaris infrared camera system (Northern Digital, Waterloo, Canada), using a headband with four passive reflective markers. A general magnetic resonance image was used to create a head model that was linked to each subject by collecting three landmarks and at least 300 additional points from the scalp with a tracking pointer. Once the ADM hotspot 
was located manually, it was added to the generated head model. A robotic arm holding the coil was used for positioning at the indicated location and displacements were automatically detected and actively corrected to ensure accurate and stable coil positioning throughout the TMS session.

\subsubsection{EEG recording and analysis}

During single and paired pulse TMS, the EEG was recorded continuously using either the NeuroCenter EEG or ASA software (Clinical Science Systems, Leiden, the Netherlands and ANT Neuro, Enschede, the Netherlands, respectively), a 64-channel EEG amplifier (TMSi, Oldenzaal, the Netherlands) and a TMS-compatible EEG cap (ANT Neuro, Enschede, the Netherlands). The ground electrode was positioned between electrode positions Fpz and Fz. The EEG signal was sampled at 4000 or $2048 \mathrm{~Hz}$ depending on the software and low-pass filtered with an anti-aliasing filter (cut-off frequency 0.2 times the sampling frequency).

The EEG was analyzed in the common average montage and down sampled to $1000 \mathrm{~Hz}$. TMS responses were first baseline corrected by subtracting the mean amplitude over a period of 350-100 ms before applying the single or conditioning pulse. Trials were defined from $50 \mathrm{~ms}$ before till $650 \mathrm{~ms}$ after each single or conditioning pulse, resulting in 50 single pulse trials and 50 paired pulse trials per ISI with a length of $700 \mathrm{~ms}$ for each electrode position. We applied single trial principal component analysis (PCA) using 40 principal components for TMS artifact reduction. For a detailed description of the PCA method, see ter Braack et al. (2013a). It is an effective method to reduce the first large artifact, which results from the magnetic pulse, as well as the second artifact, which is assumed to be caused by muscle activation on the scalp. In short, we removed the first four principal components, after which trials were filtered between $1-45 \mathrm{~Hz}$ using a fourth order Butterworth bandpass filter. By taking the average over 50 trials, the single pulse and uncorrected paired pulse TEPs were obtained for each electrode position. All TEPs were analyzed over the entire frequency range between $1-45 \mathrm{~Hz}$.

Interpretation of the paired pulse TEP is not straightforward, due to the fact that, in contrast to the MEP, the TEP is still ongoing when applying the test pulse. Thus, the early components of the response evoked by the test pulse are most likely affected by the late components of the response evoked by the conditioning pulse. Simultaneously, these same late components of the conditioning response are most likely influenced by the induced activity of the test pulse. However, these two interactions are so entangled that they cannot be disconnected (Premoli et al., 2014b). Figure 4.1 describes the paired pulse correction method we applied to correct for the first interaction: the influence of late conditioning response components on early test response components (Daskalakis et al., 2008; Premoli et al., 2014b). The corrected 
paired pulse TEP, hereafter referred to as paired pulse TEP, was obtained by subtracting the single pulse TEP from the uncorrected paired pulse TEP. Alignment of the single and test pulse enables direct comparison of the single and paired pulse TEP for the assessment of $\mathrm{LICl}$, see Figure 4.1C.

\subsubsection{Statistical analysis}

To assess $\mathrm{LICl}$ at the cortical level, the cluster-based permutation analysis (Maris and Oostenveld, 2007) was applied as implemented in FieldTrip (http://www.fieldtrip toolbox.org). This is a statistical method to analyze the spatiotemporal dynamics of multidimensional TMS-EEG data, without being affected by the multiple comparison problem. Each ISI was analyzed individually for stimulation of the dominant and non-dominant hemisphere. To compensate for handedness, topographical plots of left handed subjects were mirrored (e.g. TEPs of electrode positions C3 and C4 were exchanged). We used dependent samples $t$-tests to compare the single versus paired pulse TEPs. Comparisons were performed for each electrode position and each time sample over a period of $300 \mathrm{~ms}$ after giving the single or test pulse. Clusters were formed by $t$-values with a $p$-value $<0.05$, based on adjacent time samples and neighboring electrodes $(n \geq 2)$. To determine significance, the summed $t$-value of each cluster was statistically tested against the distribution of clusters obtained by permutation. Using the Monte Carlo method, TEPs were randomly assigned to either the single or paired pulse condition for 1500 times. Clusters found in the original data were considered to be significant if less than $5 \%$ of the summed $t$-values obtained by permutation exceeded the original cluster $t$-value, i.e. if $p$-value $<0.05$.

Comparable analyses were applied to evaluate stability of the single and paired pulse TEPs. For the effect of stimulated hemisphere, dependent samples $t$-tests were used to compare TEPs measured after stimulating the dominant versus nondominant hemisphere. For this, the topographical plots of non-dominant stimulation were mirrored. For the effect of time, TEPs of both TMS sessions were compared using dependent samples $t$-tests. Finally for the effect of coil positioning method, independent samples $t$-tests were used to compare TEPs obtained by manual versus robot-guided positioning.

Matlab (version R2015a, The Mathworks, Natick, MA, USA) was used for the statistical as well as EEG analysis. We considered a $p$-value $<0.05$ to be statistically significant and additionally adjusted for the number of tests performed (Bonferroni corrected with $\mathrm{n}=$ 2 for $\mathrm{LICl}$ and the effect of stimulated hemisphere and time, and $n=4$ for the effect of coil positioning method). Effect sizes were calculated using Cohen's $d$, defined as

$$
d=\frac{\mu_{1}-\mu_{2}}{s}
$$



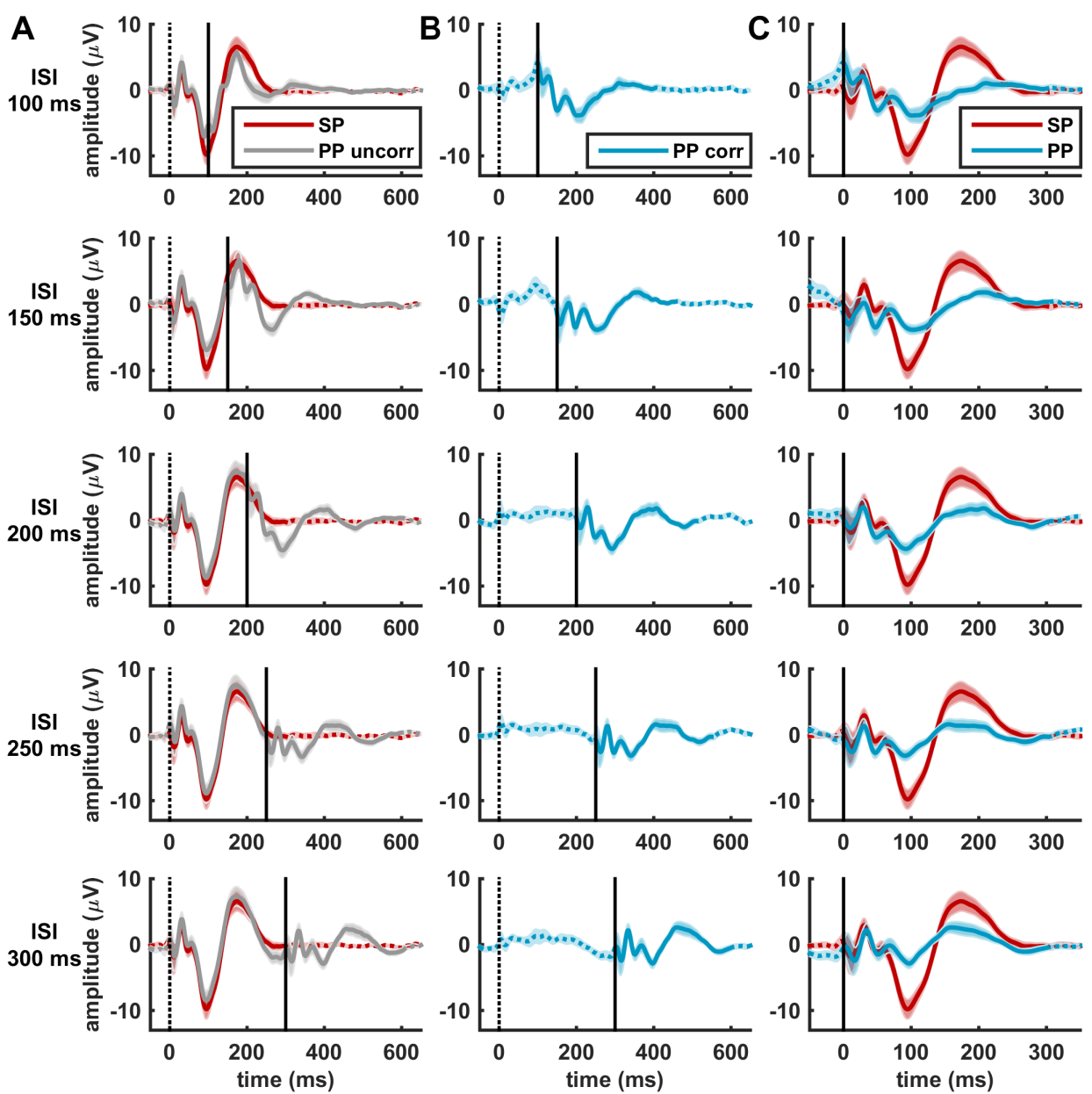

Figure 4.1: Representation of the paired pulse correction method applied to correct for the influence of late conditioning response components on early test response components. A) The single pulse TEP (SP in red) is subtracted from the uncorrected paired pulse TEP (PP uncorr in grey) to obtain $\mathrm{B}$ ) the corrected paired pulse TEP (PP corr in blue), referred to as paired pulse TEP. C) Alignment of the single pulse (SP in red) and paired pulse TEP (PP in blue) enables assessment of $\mathrm{LICl}$ at the cortical level. Especially the late N100 and P180 components are strongly suppressed for all five ISIs. Each TEP is the average over all subjects (mean \pm standard error of the mean (SEM)) at electrode position $\mathrm{Cz}$ for stimulation of the dominant hemisphere during session 1 . The moment of applying the single or conditioned pulse is indicated by the black dotted line and the moment of the test pulse by the black solid line. 
with the pooled standard deviation $s$ defined as

$$
s=\frac{\left(n_{1}-1\right) \cdot s_{1}^{2}+\left(n_{2}-1\right) \cdot s_{2}^{2}}{n_{1}+n_{2}-1} .
$$

We determined the effect size of significant clusters by taking the mean over all significant electrodes and time samples. Effect sizes above 0.8 or below -0.8 were considered to be large (Cohen, 1988).

\subsection{Results}

In sixteen of the twenty-five included subjects the coil was positioned manually, while robot-guided coil positioning was applied in nine. No adverse events happened and all participants tolerated the stimulation protocol well, except for the first excluded subject.

\subsubsection{Spatiotemporal dynamics of the single and paired pulse TEP}

The average single pulse TEP contained all the characteristic TEP components at electrode position $\mathrm{C} z$ for both stimulated hemispheres, see top panels Figure 4.2. These P30, N45, P60, N100 and P180 components were also visible in the average paired pulse TEP for ISIs between 100-300 ms, see top panels Figure 4.3. The topographical distribution of each TEP component was similar for single and paired pulse TMS, and showed a mirrored spatiotemporal dynamics for stimulation of the dominant and non-dominant hemispheres, see Figure 4.4. The P30 component is mainly expressed centrally, the N45 more frontal, the P60 near the stimulation site, the N100 centrally and the $\mathrm{P} 180$ component in centrofrontal areas.

\subsubsection{Stability of the single and paired pulse TEP}

Time had no significant effect on the single pulse TEP, see Figure 4.2. Also the paired pulse TEPs did not differ significantly between both TMS sessions. No significant clusters were found for stimulation of the dominant and non-dominant hemispheres when comparing TEPs from both TMS sessions. In addition, no significant effect of coil positioning method was found when comparing TEPs obtained by manual and robot-guided positioning; no significant clusters for both stimulated hemispheres and TMS sessions. Although we found no effect of stimulated hemisphere during the first TMS session, the paired pulse TEPs measured after stimulating the dominant and nondominant hemispheres differed twice significantly during session 2 . One significant positive cluster ( $p=0.0047, d=1.1$ ) was found for ISI $100 \mathrm{~ms}$ and one significant negative cluster ( $p<0.001, d=-1.3)$ for ISI $150 \mathrm{~ms}$. 

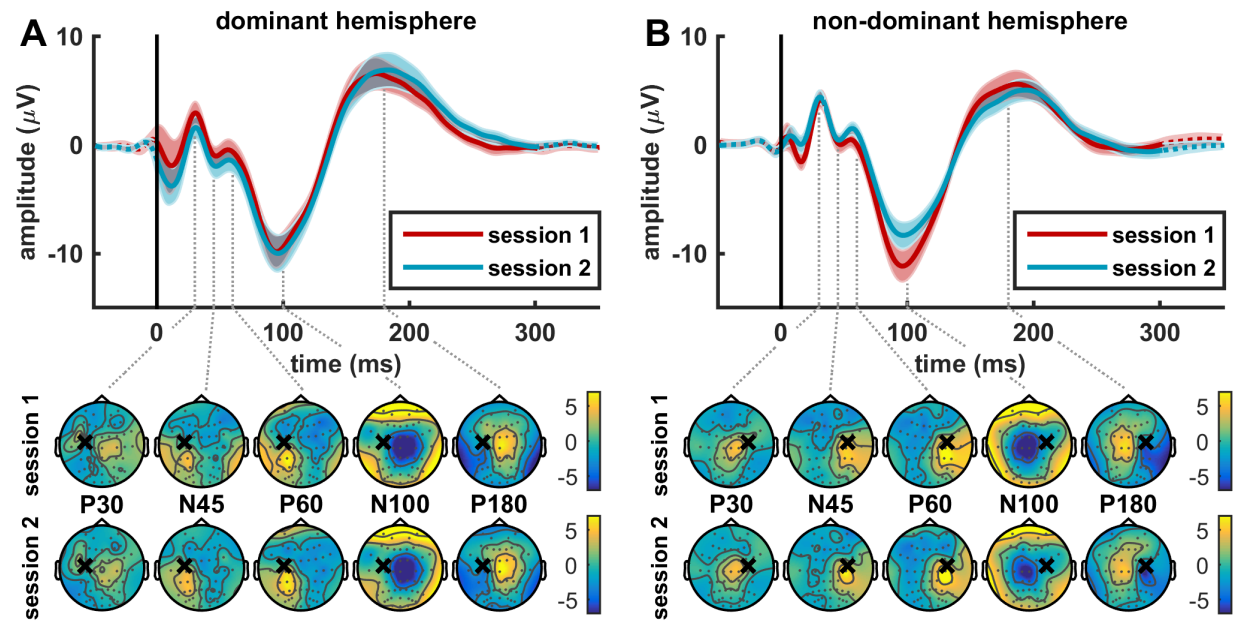

Figure 4.2: Average single pulse TEP and topographical plots of the characteristic TEP components for both TMS sessions when stimulating the A) dominant or B) non-dominant hemisphere. No significant differences were found between the single pulse TEPs of session 1 (in red) and session 2 (in blue), nor between TEPs measured after stimulating the dominant (on the left) and non-dominant (on the right) hemisphere. Each TEP is the average over all subjects (mean \pm SEM) at electrode position Cz. The topographical plots show the distribution of the P30, $\mathrm{N} 45, \mathrm{P} 60, \mathrm{~N} 100$ and P180 components, where the black cross indicates the stimulation site and the grey dots the 64 electrode positions. Yellow areas indicate a positive amplitude and blue areas a negative amplitude. 

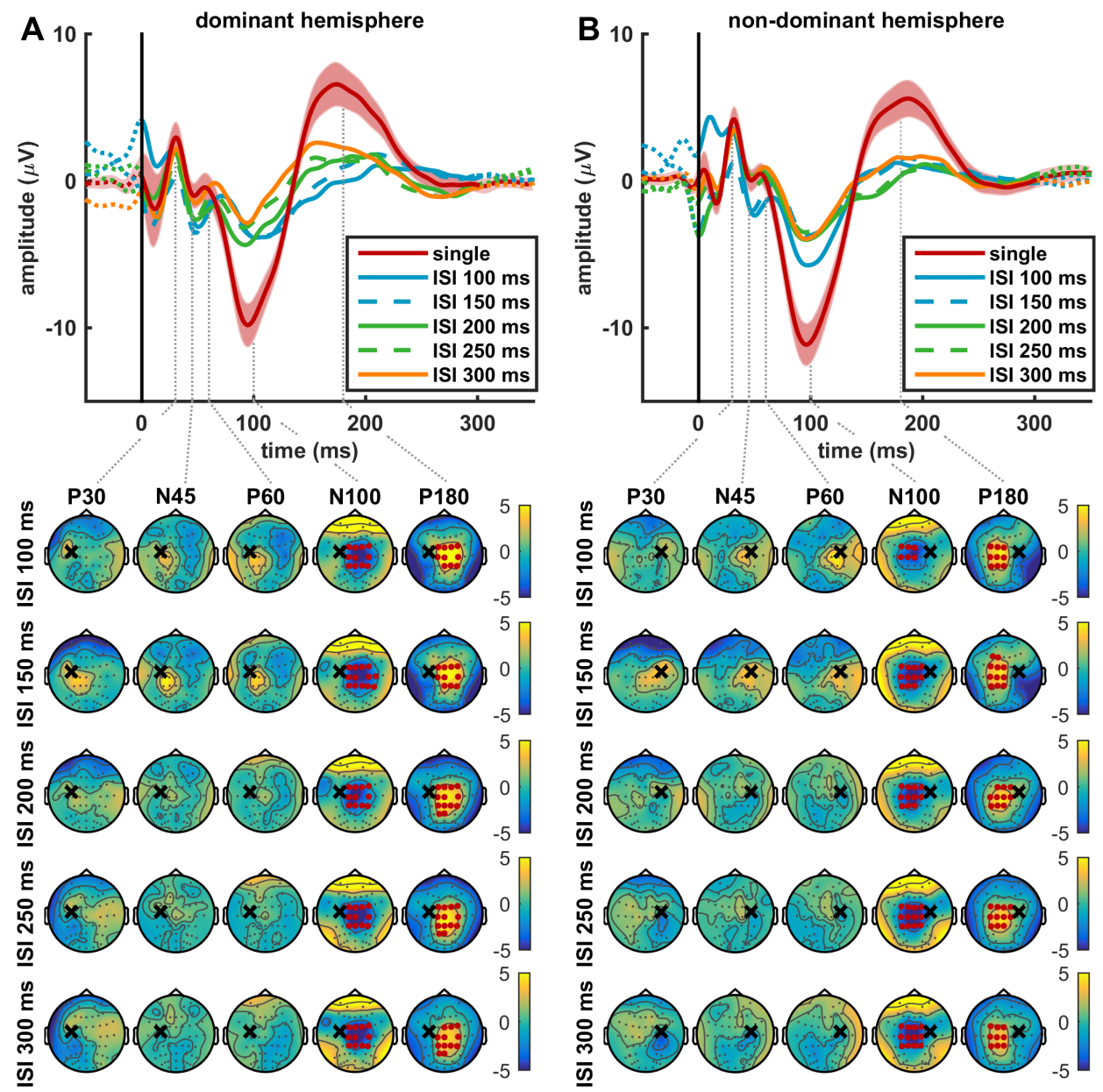

Figure 4.3: Average single and paired pulse TEPs and topographical LICI plots of the characteristic TEP components when stimulating the A) dominant or B) non-dominant hemisphere during session 1 . We assessed $\mathrm{LICl}$ by comparing the single versus paired pulse TEP. The late N100 and P180 components are strongly suppressed for all five ISIs, while the early P30, N45 and P60 components remained unaffected. Significant negative N100 and positive P180 clusters were found in central areas, corresponding to inhibition at the expression site of the late components. The single pulse TEP is the average over all subjects (mean \pm SEM) at electrode position $\mathrm{Cz}$, while for each paired pulse TEP only the mean is presented. The topographical plots show the distribution of $\mathrm{LICl}$ for the P30, N45, P60, N100 and P180 components. The black cross indicates the stimulation site, the grey dots the 64 electrode positions and the red dots electrodes that show a significant difference between single and paired pulse TEPs. Yellow areas indicate inhibition of positive amplitudes and blue areas inhibition of negative amplitudes. 
A
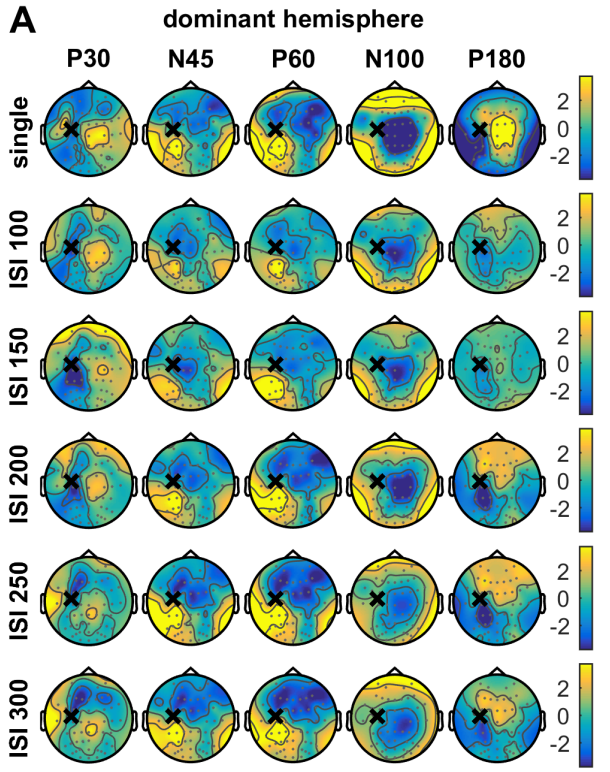
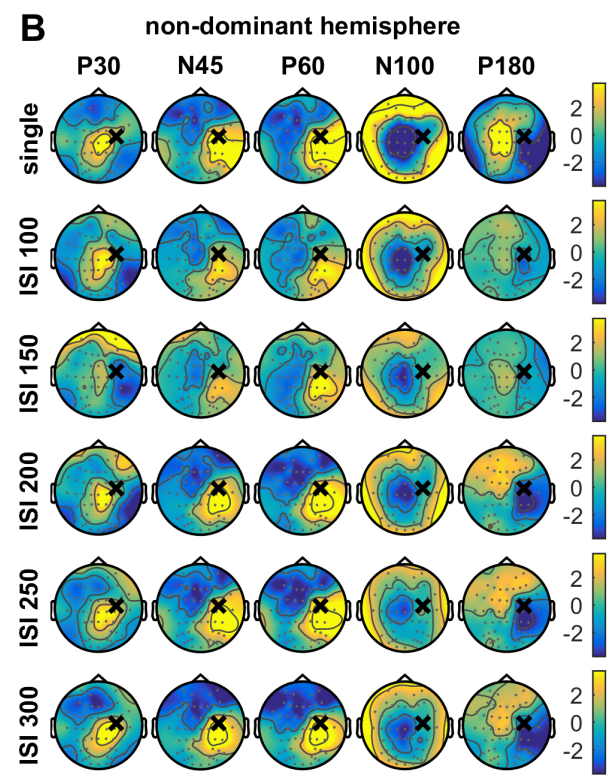

Figure 4.4: Topographical distribution of the characteristic P30, N45, P60, N100 and P180 components when stimulating the $\mathrm{A}$ ) dominant or $\mathrm{B}$ ) non-dominant hemisphere during session 1. Spatiotemporal dynamics was similar for single and paired pulse TMS, and stimulation of the dominant and non-dominant hemispheres resulted in a mirrored pattern. The black cross indicates the stimulation site and the grey dots the 64 electrode positions. Yellow areas indicate a positive amplitude and blue areas a negative amplitude. 


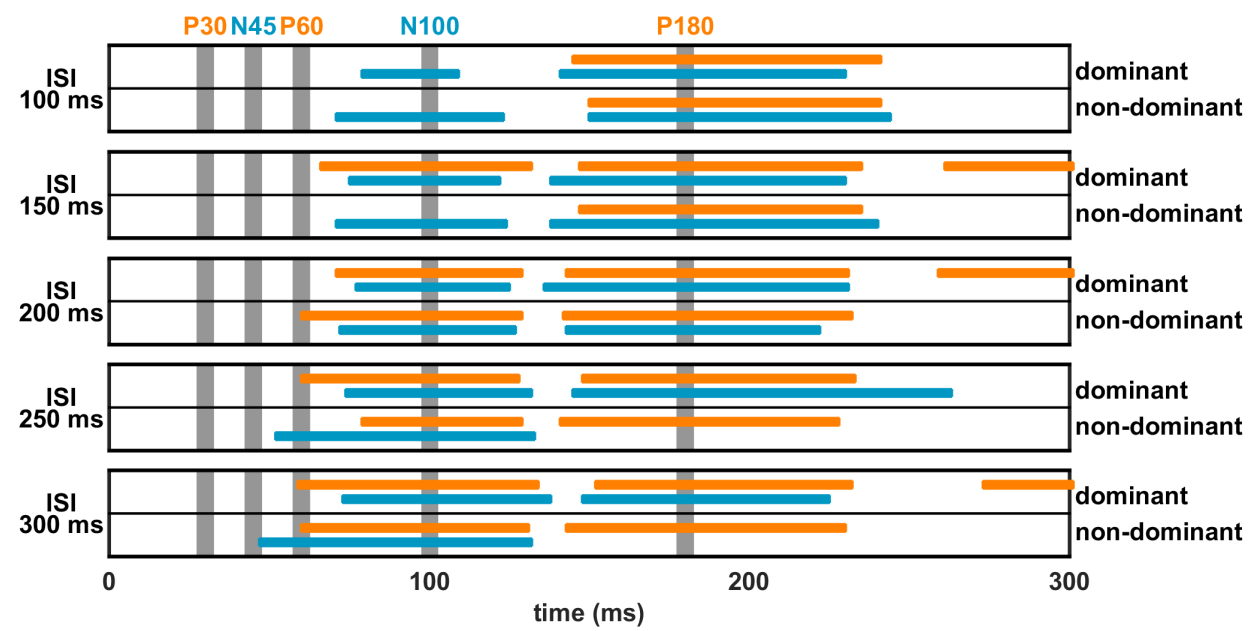

Figure 4.5: Overview of all significant LICI clusters (single versus paired pulse TEP) found when stimulating the dominant and non-dominant hemispheres during session 1. Positive clusters are presented in orange indicating inhibition of positive amplitudes and negative clusters in blue indicating inhibition of negative amplitudes. Besides the negative $\mathrm{N} 100$ and positive P180 clusters, also significant positive $\mathrm{N} 100$ and negative $\mathrm{P} 180$ clusters were found, as well as positive late clusters. The vertical grey bars indicate the time corresponding to the P30, N45, P60, N100 and P180 components.

\subsection{3 $\mathrm{LICl}$ of the paired pulse TEP}

Since we found no significant effect of time on both the single and paired pulse TEP, only $\mathrm{LICl}$ outcomes of session 1 are evaluated and presented. Compared to the single pulse TEP at electrode position $\mathrm{Cz}$, we found strong suppression of the late paired pulse TEP components (N100 and P180), while the early components (P30, N45 and P60) remained unaffected, see Figure 4.1C and Figure 4.3. Assessment of $\mathrm{LICl}$ (single versus paired pulse TEP) showed a significant negative N100 and positive P180 cluster at all ISIs between 100-300 ms for stimulation of both the dominant and nondominant hemisphere (negative N100 clusters: $p \leq 0.005$, range $d$ : $-1.0--1.5$; positive P180 clusters: $p \leq 0.001$, range $d: 1.0-1.8)$. However, no significant clusters were found for the P30, N45 and P60 components, see Figure 4.3. Topographical LICI plots showed significant inhibition of the $\mathrm{N} 100$ and P180 for the central areas, corresponding to the expression site of these components. No clear differences were seen in $\mathrm{LICl}$ expression between the five ISIs. In addition to the negative N100 and positive P180 clusters, we often also found significant positive $\mathrm{N} 100$ ( $p \leq 0.01$, range $d$ : $0.9-1.6)$ and negative P180 clusters ( $p \leq 0.003$, range $d:-1.1--2.1$ ), as well as late positive clusters ( $p \leq 0.01$, range $d: 1.0-1.3$ ) around $280 \mathrm{~ms}$, see Figure 4.5. The positive N100 and negative P180 clusters were located bilaterally in occipito-temporal areas and the late 
positive clusters occipitally on the contralateral side. For a complete overview of the spatiotemporal dynamics of $\mathrm{LICl}$, see the movie in the Supplementary material.

\subsection{Discussion}

In this study we evaluated the spatiotemporal dynamics and stability of the single and paired pulse TEP. The topographical distribution of the P30, N45, P60, N100 and P180 components was similar for single and paired pulse TMS. Stimulation of the dominant and non-dominant hemispheres resulted in a mirrored spatiotemporal dynamics. TMS-EEG outcomes appear to be stable, as single and paired pulse TEPS were not significantly affected by either stimulated hemisphere, time or coil positioning method. In addition, we assessed $\mathrm{LICl}$ at the cortical level for ISIs between 100-300 ms by comparing the single versus paired pulse TEP. For all ISIs, it was characterized by significant suppression of the late $\mathrm{N} 100$ and P180 components in the central areas, without affecting the early P30, N45 and P60 components.

Inhibition of the N100 and P180 components was found consistently for all five ISIs. Accordingly, Opie et al. (2017) found no significant differences in LICl expression for ISIs 100 and $150 \mathrm{~ms}$. More remarkable, TEP modulation was also largely identical for $\mathrm{SICI}$ (ISI $2 \mathrm{~ms}$ ) and LICI (ISI $100 \mathrm{~ms}$ ) (Premoli et al., 2018). These findings indicate an overarching inhibitory mechanism regardless of ISI, in contrast to pharmacoTMS-EMG studies showing two distinct inhibitory mechanisms. $\mathrm{SICl}$ is associated with GABA-A receptor mediated inhibition (Di Lazzaro et al., 2007; Kujirai et al., 1993), while $\mathrm{LICl}$ reflects GABA-B receptor mediated inhibition (McDonnell et al., 2006; Müller-Dahlhaus et al., 2008). Thus, it appears that knowledge obtained with the TMS-EMG paradigm is not directly translatable to $\mathrm{SICl}$ and $\mathrm{LICI}$ measured with TMS-EEG (Opie et al., 2017; Premoli et al., 2018). Single pulse pharmaco-TMS-EEG studies demonstrated that the $\mathrm{N} 45$ and N100 components are related to GABA-A and GABA-B receptor mediated inhibition, respectively (Premoli et al., 2014a). The fact that we found inhibition of the $\mathrm{N} 100$ component when assessing $\mathrm{LICl}$, points more to GABA-B than GABA-A receptor mediated inhibition. However, more paired pulse pharmaco-TMS-EEG studies, focusing on SICI and $\mathrm{LICl}$ are needed to increase our understanding of the underlying inhibitory mechanisms involved in TMS-EEG.

Our result of significant $\mathrm{LICl}$ of only the late N100 and P180 components, corresponds with recent findings for $\mathrm{SICl}$ (Premoli et al., 2018), supporting the hypothesis of an overarching inhibitory mechanism. However, it contradicts with previous findings of suppression of both early and late TEP components at ISI $100 \mathrm{~ms}$ (Opie et al., 2017, 2018; Premoli et al., 2014b) and a significant decrease of the N40, N100 and P180 components at ISI 150 ms, but not the P30 (Opie et al., 2017, 2018). Discrepancies might be partly 
due to differences in stimulation intensity. Rogasch et al. (2013) showed that increasing the test pulse intensity results in decreased $\mathrm{LICl}$ of the early P30 and P60 components, without affecting later components. The fact that we stimulated at an intensity of $120 \%$ rMT, instead of 100\% rMT like Premoli et al. (2014b), could explain that no significant clusters were found for the P30, N45 and P60 components. In addition, inconsistencies might have occurred because we evaluated the entire TEP response over a period of $300 \mathrm{~ms}$, instead of focusing on shorter periods around the TEP components.

The majority of significant clusters could be assigned to one of the characteristic TEP components, suggesting that they contain most of the essential information regarding $\mathrm{LICl}$. However, when stimulating the dominant hemisphere we additionally found a late positive cluster around $280 \mathrm{~ms}$ for ISIs 150, 200 and $300 \mathrm{~ms}$. Although the relevance of this late cluster remains speculative, source localization techniques may assist in elucidating its origin. Furthermore, we recommend that analysis of TMS-EEG data includes evaluation of the late TEP response, as single pulse TMS-EEG studies showed the potential of late responses as a biomarker for epilepsy (Shafi et al., 2015; Valentin et al., 2008). Source localization can also help to gain insight into the presence and distribution of dipoles. Just as Opie et al. (2017, 2018), we often found positive N100 and negative P180 clusters in occipito-temporal areas in addition to the central clusters, indicating an underlying dipole.

Before evaluating the paired pulse TEP, we applied a method to correct for the influence of late conditioning response components on early test response components (Daskalakis et al., 2008; Premoli et al., 2014b). However, this method did not take into account the likely modulation of late conditioned response components caused by giving the test pulse (Premoli et al., 2014b). The fact that it is not possible to correct for both interactions due to their entanglement, makes this paired pulse correction method suboptimal. As an alternative, this study suggests stimulation at ISI $300 \mathrm{~ms}$ when assessing $\mathrm{LICl}$ at the cortical level, instead at the most commonly used ISI $100 \mathrm{~ms}$. $\mathrm{LICl}$ expression was similar for both ISIs, just as the topographical distribution of TEP components. More importantly, where the conditioning pulse TEP is still ongoing when applying the test pulse after $100 \mathrm{~ms}$, EEG activity has visually returned to baseline at $300 \mathrm{~ms}$, see Figure 4.1. Even though this may not apply to the underlying inhibitory mechanisms, paired pulse correction seems redundant at ISI $300 \mathrm{~ms}$. This implies that paired pulse stimulation at ISI $300 \mathrm{~ms}$ (or longer) allows assessment of $\mathrm{LICl}$, without the need of using any correction method.

Clinical applicability of TMS-EEG, as well as the ability to use our findings from healthy subjects as reference values for future TMS-EEG studies, depend on the stability of the single and paired pulse TEP. In this study, we demonstrated the stability and robustness of the single and paired pulse TEP in a rather heterogeneous group of 
healthy subjects. In line with previous studies, spatiotemporal dynamics of the paired pulse TEP showed great resemblance between ISIs (Opie et al., 2017, 2018) and was similar to the single pulse TEP (Bonato et al., 2006; Ferreri et al., 2011; Paus et al., 2001; Premoli et al., 2014a). Stimulation of the dominant and non-dominant hemisphere resulted in a mirrored pattern without significantly affecting the TEPs, except for ISIs 100 and 150 ms during session 2. In addition, we confirmed the high reproducibility of both single (Casarotto et al., 2010; Lioumis et al., 2009) and paired pulse TEPs after one week (Farzan et al., 2010; Premoli et al., 2014b). Previously, our group already showed good repeatability of the single pulse TEP during the day (ter Braack et al., 2018). Furthermore, manual coil positioning seems sufficient given the limited added value of robot-guided positioning. In agreement with our earlier TMSEMG findings on a group level (de Goede and van Putten, 2017), no significant effect was found for stimulated hemisphere, time and coil positioning method demonstrating the robustness of TMS-EEG.

This study is limited by the fact that we only evaluated the effect of stimulated hemisphere, time and coil positioning method. However, there are more factors that need to be investigated because they may influence the single and paired pulse TEP, like pulse waveform, stimulation intensity, age, mental state or coil orientation and tilt.

Another limitation is that we applied single trial PCA to reduce the first large TMS artifact and second muscle artifact in our EEG data. PCA has shown to be an effective method to reduce both artifacts simultaneously (ter Braack et al., 2013a; Rogasch et al., 2017). However, EEG data can be contaminated by other artifacts as well, such as eyeblinks, auditory and somatosensory artifacts (Ilmoniemi and Kičić, 2010; Ilmoniemi et al., 2015). Thus, a combination of multiple methods, including PCA, seems more appropriate to remove all types of artifacts without significantly affecting the TEP. Even though we strongly encourage the recent development of (fully) automated artifact rejection algorithms (Atluri et al., 2016; Rogasch et al., 2017; Wu et al., 2018), they are mainly tested on the single pulse TEP. More research is needed to make these algorithms suitable for paired pulse TMS-EEG data. In addition, we always removed the first four principal components, while it may be better to manually determine and adjust this number per subject (ter Braack et al., 2013a).

\subsection{Conclusion}

$\mathrm{LICl}$ at the cortical level was characterized by significant suppression of the late N100 and P180 components, without affecting the early P30, N45 and P60 components. As no differences in $\mathrm{LICl}$ expression were found between ISIs, stimulation at ISI $300 \mathrm{~ms}$ is preferred because paired pulse correction seems superfluous here. Despite strong 
inhibition at the expression site of the late TEP components, the topographical distribution of the characteristic components was similar for the single and paired pulse TEP. These spatiotemporal dynamics in healthy subjects can serve as reference values for future neuropsychiatric and pharmacological studies, as cortical excitability is modified for various central nervous system conditions and central acting drugs. We found no significant effect of stimulated hemisphere, time and coil positioning method, demonstrating the robustness and stability of TMS-EEG outcomes as a potential clinical biomarker.

\section{Acknowledgments}

We thank all the subjects for their participation. Furthermore, we would like to thank Carin Eertman for her assistance during the TMS measurements and Anna Dietze and Michelle van Mierlo for their help with the cluster-based permutation analysis.

\section{Funding}

This work was supported by the Dutch TWIN foundation for neuromodulation. The funding source played no role in the design of the study, collection, analysis and interpretation of the data, and writing of the manuscript.

\section{Disclosures}

M.J.A.M. van Putten is co-founder of Clinical Science Systems, Leiden, the Netherlands.

\section{Supplementary material}

See online version of the movie at https://www.youtube.com/watch?v=ka3a2l7p0k4 or scan the QR code.

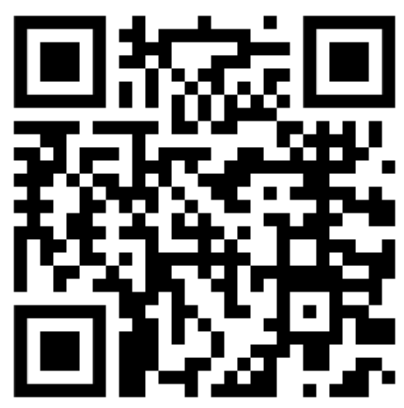





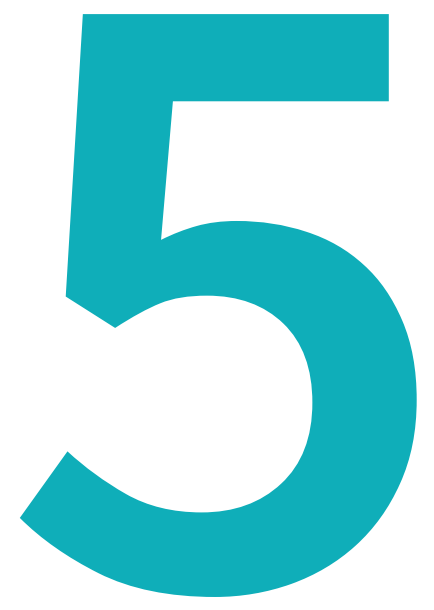

\section{Infraslow activity as a potential modulator of cortical excitability}

A.A. de Goede and M.J.A.M. van Putten

Submitted in revised form: Journal of Neurophysiology 


\section{Abstract}

Objective: Fluctuations in cortical excitability are a candidate mechanism involved in the trial-to-trial variation of motor evoked potentials (MEPs) to transcranial magnetic stimulation (TMS). We explore whether infraslow electroencephalography (EEG) activity $(<0.1 \mathrm{~Hz})$ modulates cortical excitability by evaluating the presence of temporal and phase clustering of TMS induced MEPs. In addition, we evaluate the dependence of MEP amplitude on the phase of the infraslow activity.

Methods: Twenty-three subjects were stimulated at an intensity above the resting motor threshold (rMT) and ten at the rMT. We evaluated if temporal and phase clustering of MEP size and MEP generation was present using 1000 surrogates with a similar amplitude or occurrence distribution. To evaluate the MEP amplitude dependence, we used the least-square method to approximate the linear circular data by fitting a sine function.

Results: We observed significant temporal clustering in all subjects stimulated at the rMT and in the majority stimulated above rMT, suggesting underlying determinism of cortical excitability instead of randomly generated fluctuations. The majority of subjects showed significant phase clustering for MEP size and for MEP occurrence. Furthermore, in approximately a quarter to half of the subjects we found a significant correlation and dependence of MEP amplitude on the phase of infraslow activity, respectively.

Conclusion: While other mechanisms most likely contribute as well, our findings suggest that infraslow activity is involved in the variability of cortical excitability and TMS induced responses.

New \& Noteworthy: Cortical excitability measures are highly variable during TMS. Although ongoing brain oscillations are assumed to modulate excitability, no consistent associations are found for the traditional frequency bands. We focus on the role of infraslow EEG activity, defined as rhythms with frequencies $<0.1 \mathrm{~Hz}$. We provide experimental evidence suggesting that infraslow activity modulates cortical excitability and that response variation could be reduced when stimulation is targeted at a specific phase of infraslow activity. 


\subsection{Introduction}

Cortical excitability, defined as the strength of a particular cortical output in response to an external stimulus, can be assessed non-invasively by transcranial magnetic stimulation (TMS). Single pulse TMS at the motor cortex can induce a motor evoked potential (MEP), of which the peak-to-peak amplitude is a measure of cortical excitability (Barker et al., 1985). This amplitude is not constant when stimulating multiple times at the same intensity (Goldsworthy et al., 2016; Hess et al., 1987; Kiers et al., 1993; Roy Choudhury et al., 2011). While this may result from variations in experimental design, such as minor changes in coil position or experimental noise, biological variations are likely to be involved as well (Schmidt et al., 2015). A candidate mechanism responsible for these trial-to-trial variations are fluctuations in cortical excitability (Amassian et al., 1989a; Ferreri et al., 2014; Kiers et al., 1993).

Several studies suggest that ongoing brain oscillations are an important modulator of cortical excitability (Berger et al., 2014; Ferreri et al., 2014; Iscan et al., 2016; Kundu et al., 2014; Mäki and Ilmoniemi, 2010a; Sauseng et al., 2009; Schulz et al., 2014; Zarkowski et al., 2006; Zrenner et al., 2018). Whereas some report a correlation between the MEP amplitude and the pre-stimulus power in either the alpha (Sauseng et al., 2009; Zarkowski et al., 2006), beta (Mäki and IImoniemi, 2010a; Schulz et al., 2014) or gamma band (Zarkowski et al., 2006), others fail to find significant correlations for the various frequency bands (Berger et al., 2014; Iscan et al., 2016; Zrenner et al., 2018). Instead of pre-stimulus power, a recent study suggests that the high-alpha power variability might be a better predictor of variations in MEP amplitude (Iscan et al., 2016). Other studies found a significant association between MEP amplitude and the instantaneous phase of mu-oscillations in the alpha band (Zrenner et al., 2018), or of oscillations in the midrange-beta band (Mäki and IImoniemi, 2010a) or in the alpha, fast-beta and gamma bands (Berger et al., 2014).

Although brain oscillations are assumed to modulate cortical excitability, no consistent associations are found for the traditional frequency bands, ranging from delta to gamma (Berger et al., 2014; Iscan et al., 2016; Mäki and Ilmoniemi, 2010a; Sauseng et al., 2009; Schulz et al., 2014; Zarkowski et al., 2006; Zrenner et al., 2018). However, the brain also generates activity below $0.1 \mathrm{~Hz}$ and far above $70 \mathrm{~Hz}$ (Aladjalova, 1964; Bragin et al., 1999; Vanhatalo et al., 2004). Brain rhythms with frequencies below $0.1 \mathrm{~Hz}$ are termed infraslow activity, or infraslow oscillations if they have a (nearly) periodic character (Aladjalova, 1957, 1964; Hughes et al., 2011). Infraslow activity was measured for the first time in rabbits by Aladjalova in 1957. She observed faster oscillations that were phase-locked to the rising phase of the infraslow oscillation, suggesting that infraslow activity modulates cortical excitability (Aladjalova, 1957). This was supported by her 
finding that cortical responses to afferent stimulation were primarily evoked when cortical excitability was increased and not when it was decreased.

More recently, Vanhatalo et al. (2004) showed that during sleep the phase of infraslow oscillations clearly correlates with both the amplitude of higher frequencies $(1-100 \mathrm{~Hz})$, as well as the occurrence of $\mathrm{K}$-complexes and interictal epileptiform discharges (Vanhatalo et al., 2004). Also in awake subjects, the amplitude of faster oscillations $(1-40 \mathrm{~Hz})$ is strongly related to the infraslow fluctuation phase. In addition, the ability to detect a sensory stimulus significantly depends on the phase of infraslow fluctuations, but not on the amplitude (Monto et al., 2008). Infraslow oscillations are even observed in postanoxic encephalopathy and in those patients with a burstsuppression pattern, bursts are phase-locked to the phase of the infraslow activity (van Putten et al., 2015). These observations further support the notion that infraslow activity appears to be a modulator of cortical excitability and of importance for physiological and pathological brain function (Hughes et al., 2011; van Putten et al., 2015; Vanhatalo et al., 2004).

The only TMS study examining the association between infraslow oscillations and variations in MEP amplitude, was performed during non-rapid eye movement (NREM) sleep. More supra-threshold MEPs (amplitude $>50 \mu \mathrm{V}$ ) were evoked during the rising state of the infraslow oscillation, than during the falling state. In addition, MEP amplitudes were approximately $20 \%$ larger when evoked during the rising state. The more positive the rising state or the less negative the falling state, the larger the MEP amplitude (Bergmann et al., 2012). Obtaining similar findings in awake subjects might provide possibilities to reduce the large variation in MEP amplitude, as stimulation can be targeted at a specific oscillatory phase.

We use TMS evoked potentials to study if oscillations in infraslow activity modulate cortical excitability. We focus on the relation between infraslow activity and 1) the MEP amplitude, by stimulating above the resting motor threshold (rMT) and 2) the occurrence of a MEP, by stimulating at the rMT.

\subsection{Materials and methods}

We used single pulse TMS data that was collected as part of two larger trials (trial IDs: NL36317.044.11 and NL49854.044.14). Both study protocols were approved by the medical ethics committee (Medisch Spectrum Twente, Enschede, the Netherlands) and were in accordance with the Declaration of Helsinki. In addition, we followed the guidelines for the use of TMS in clinical practice and research (Rossi et al., 2009). Part of the dataset was previously used in another context by ter Braack et al. (2013a, 2016) and by de Goede and van Putten (2017). 


\subsubsection{Subjects}

Healthy adults (18 years or older) were included after they gave written informed consent. Subjects with contraindications as mentioned in the Screening Questionnaire before TMS (Rossi et al., 2011) were excluded. Subjects either participated in the study evaluating the relation between infraslow activity and MEP amplitude, or in the study focusing on the relation between infraslow activity and MEP occurrence.

\subsubsection{TMS protocol}

During stimulation, subjects were instructed to keep their eyes open and to hold their dominant hand pronated in a relaxed position. Handedness was determined using the Dutch Handedness Questionnaire (van Strien, 1992, 2003).

In all subjects, we manually located the motor hotspot of the abductor digiti minimi (ADM) muscle in the dominant hemisphere (left hemisphere for right-handedness). A figure-of-eight air film $70 \mathrm{~mm}$ coil (The Magstim Company Ltd, Whitland, United Kingdom) was placed tangentially at the ADM hotspot, with the handle pointing backwards and laterally at an angle of $45^{\circ}$ from the midline. Single biphasic TMS pulses, with a pulse duration of $400 \mu \mathrm{s}$, were given using a Magstim Rapid ${ }^{2}$ Stimulator (The Magstim Company Ltd, Whitland, United Kingdom). The stimulation intensity depended on the rMT, which was defined as the minimum intensity needed to evoke at least five MEPs, with an amplitude of at least $50 \mu \mathrm{V}$, out of ten consecutive pulses (Groppa et al., 2012; Rossini et al., 2015).

\section{Relation between infraslow activity and MEP amplitude}

Some subjects were stimulated with 50 single pulses at an intensity of $120 \%$ rMT, with a random interpulse interval between $3.5-4.5 \mathrm{~s}$. In others, 75 single pulses were given at an intensity of $110 \% \mathrm{rMT}$, with a random interpulse interval between 3-4 $\mathrm{s}$.

\section{Relation between infraslow activity and MEP occurrence}

Subjects were stimulated with 200 single pulses at the rMT, to provoke that MEPs were only evoked $50 \%$ of the time. Pulses were divided into four blocks of 50 , so that the stimulation intensity could be slightly adjusted if the rMT showed drift between blocks. A random interval ranging from 9.5-10.5 s was kept between consecutive single pulses.

\subsubsection{EEG recording and analysis}

Full-band EEG was continuously recorded during single pulse TMS using either the NeuroCenter EEG or ASA software (Clinical Science Systems, Leiden, the Netherlands and ANT Neuro, Enschede, the Netherlands, respectively), a DC-coupled EEG amplifier (TMSi, Oldenzaal, the Netherlands) and a TMS-compatible EEG cap (ANT Neuro, 
Enschede, the Netherlands). The EEG was sampled at either 4000 or $2048 \mathrm{~Hz}$, with the ground electrode located between electrode positions Fz and Fpz. The sample frequency of $2048 \mathrm{~Hz}$ was only used when stimulating at $110 \%$ rMT.

For analysis we selected the electrode closest to the stimulation location (electrodes $\mathrm{C} 3$ and $\mathrm{C} 4$ for the left and right hemispheres, respectively), referenced to electrode position $\mathrm{Cz}$. The TMS artifact was removed by linear interpolation over a period of $25 \mathrm{~ms}$ around the TMS pulse. Thereafter, the EEG data was filtered with a first order Butterworth bandpass filter between $0.01-0.1 \mathrm{~Hz}$, to evaluate the infraslow activity. We extracted the phase of the infraslow activity at the moment of stimulation using the Hilbert transform in Matlab (version R2015a, The Mathworks, Natick, MA, USA).

\subsubsection{EMG recording and analysis}

Two surface $\mathrm{Ag} / \mathrm{AgCl}$ electrodes placed in a belly-tendon montage were used to measure the ADM muscle activity. For recording we either used the bipolar inputs on the EEG amplifier or an additional amplifier coupled to the EEG amplifier (both from TMSi, Oldenzaal, the Netherlands). In the first case the EMG was sampled at $4000 \mathrm{~Hz}$ and the EEG ground electrode was used, while in the second case the sample frequency was $2048 \mathrm{~Hz}$ and an external ground electrode was placed on the dorsal side of the nondominant hand.

Even though subjects were asked to fully relax their ADM muscle, recordings were afterwards checked for muscle pre-activation. Trials containing EMG activity larger than $50 \mu \mathrm{V}$ in the $50 \mathrm{~ms}$ preceding the pulse were excluded. Thereafter, we calculated the peak-to-peak amplitude of the MEP.

\subsubsection{Statistical analysis}

To evaluate temporal clustering beyond chance, we investigated for each subject whether a non-random pattern was visible over time in MEP size or MEP occurrence. For stimulation above the rMT, MEPs were divided into the ones having a 'large' or 'small' amplitude using the $50^{\text {th }}$ percentile per subject. For stimulation at the rMT, responses were divided based on whether a MEP was 'present' or 'absent' using an amplitude threshold of $50 \mu \mathrm{V}$. The pattern formed by time periods of consecutive equal responses (TP-ER) was compared to the patterns obtained using Monte Carlo simulation. For each subject, we created 1000 surrogates based on the recorded data. The measured responses were shuffled using random permutation without replacement, resulting in patterns with a similar large/small or present/absent distribution. Per subject, we defined the mean length of TP-ER and the number of TP-ER for the recorded data as well as the average length and number of TP-ER over the 1000 surrogates. Paired samples 
$t$-tests ( $\alpha=0.05$, two-sided) were used to determine if there was a significant difference in mean length and number of TP-ER between subjects and surrogates. In addition, we calculated the probability of TP-ER as a function of their length for the recorded data as well as for the 1000 surrogates. Significant temporal clustering was assumed if the probability curve fell outside the 5-95 percentile range of the surrogate data, i.e. $5^{\text {th }}$ percentile of surrogates $>$ probability $>95^{\text {th }}$ percentile of surrogates.

Complementary to temporal clustering, we investigated for each subject the pattern in MEP size ('large' or 'small') and MEP occurrence ('present' or 'absent') as a function of the phase of the infraslow activity, to evaluate the presence of significant phase clustering. The pattern formed by phase periods of consecutive equal responses (PP-ER) was compared to the patterns obtained using Monte Carlo simulation with 1000 surrogates. Again, surrogates were created using random permutation without replacement and we calculated the average length and number of PP-ER over the 1000 surrogates. Due to the periodic nature of the phase, PP-ER could be formed by equal responses at the beginning and end of the phase period $-\pi$ to $\pi$. We used paired samples $t$-tests ( $\alpha=0.05$, two-sided) to test for a significant difference in mean length and number of PP-ER between subjects and surrogates. For both the recorded data and the surrogates we calculated the probability of PP-ER as a function of their length. Significant phase clustering was assumed if the PP-ER probability curve fell outside the 5-95 percentile range of the surrogates.

In addition to the binary approach of MEP amplitude ('large' or 'small'), we investigated the relation between the size of the MEP amplitude and phase of the infraslow activity. We assumed that the relation between the linear (MEP amplitude) and circular data (phase of infraslow activity) can be described by the function:

$$
\text { MEPamplitude }=a+b \cdot \sin (\theta-c) \text {. }
$$

The larger the value of parameter $b$, the stronger MEP amplitude depends on the phase. The least-square method was used for curve fitting and to determine the correlation. Again, we used a Monte Carlo simulation (random permutation without replacement) with 1000 surrogates to determine the significance of parameter $b$ and correlation coefficient $R^{2}$. We assumed significant phase dependence if parameter $b$ fell outside the 5-95 percentile range of surrogates and significant correlation if $R^{2}>95^{\text {th }}$ percentile of surrogates.

\subsection{Results}

Previously collected single pulse data of thirty-eight subjects was considered suitable for this study. Nevertheless, five subjects were excluded from analysis: three subjects 
Table 5.1: Overview of the estimated rMT values and applied stimulation intensities for subjects stimulated at three intensities: $120 \%$ rMT (nrs. 1-13), 110\% rMT (nrs. 14-23) and 100\% rMT (nrs. 24-33). Both rMT and TMS intensity are expressed as the percentage of maximum stimulator output $(0.8 \mathrm{~T})$.

\begin{tabular}{|c|c|c|c|c|c|c|c|c|c|c|c|c|c|}
\hline \multicolumn{14}{|c|}{ Stimulation intensity $120 \%$ rMT } \\
\hline Subject & 1 & 2 & 3 & 4 & 5 & 6 & 7 & 8 & 9 & 10 & 11 & 12 & 13 \\
\hline rMT (\%) & 62 & 59 & 59 & 70 & 68 & 70 & 58 & 79 & 76 & 64 & 68 & 75 & 61 \\
\hline Intensity (\%) & 74 & 71 & 71 & 84 & 82 & 84 & 70 & 95 & 91 & 77 & 82 & 90 & 73 \\
\hline \multicolumn{14}{|c|}{ Stimulation intensity $110 \%$ rMT } \\
\hline Subject & 14 & 15 & 16 & 17 & 18 & 19 & 20 & 21 & 22 & 23 & & & \\
\hline rMT (\%) & 87 & 80 & 70 & 86 & 80 & 88 & 74 & 77 & 84 & 74 & & & \\
\hline Intensity (\%) & 96 & 88 & 77 & 95 & 88 & 97 & 82 & 85 & 93 & 82 & & & \\
\hline \multicolumn{14}{|c|}{ Stimulation intensity $100 \%$ rMT } \\
\hline Subject & 24 & 25 & 26 & 27 & 28 & 29 & 30 & 31 & 32 & 33 & & & \\
\hline rMT (\%): block 1 & 62 & 51 & 58 & 56 & 57 & 40 & 58 & 63 & 64 & 60 & & & \\
\hline rMT (\%): block 2 & 56 & 48 & 61 & 59 & 60 & 40 & 57 & 63 & 55 & 65 & & & \\
\hline rMT (\%): block 3 & 60 & 52 & 60 & 53 & 56 & 41 & 58 & 61 & 58 & 65 & & & \\
\hline rMT (\%): block 4 & 61 & 55 & 59 & 56 & 53 & 41 & 57 & 61 & 59 & 64 & & & \\
\hline
\end{tabular}

showed a lot of muscle pre-activation and in two subjects MEPs were often not evoked at an intensity of $110 \%$ rMT. We included thirteen subjects ( 2 males, mean age $28 \pm$ 9.0 years; range $20-49$ years, 12 right-handed) that were stimulated at an intensity of $120 \%$ rMT, ten subjects ( 8 males, mean age $29 \pm 10.4$ years; range $21-54$ years, all right-handed) stimulated at $110 \%$ rMT and ten subjects ( 6 males, mean age $29 \pm 9.2$ years; range 23-54 years, 7 right-handed) stimulated at the rMT. For an overview of the estimated rMT values and applied stimulation intensities, see Table 5.1. All participants tolerated the single pulse protocol well and there were no adverse events.

All subjects showed infraslow activity in the $0.01-0.1 \mathrm{~Hz}$ range, with peak amplitudes larger than $5 \mu \mathrm{V}$. Four examples are presented in Figure 5.1A-D, including the phase of the infraslow activity and MEPs.

\subsubsection{Stimulation intensity $120 \%$ rMT}

The bottom row of Figure 5.2A shows the pattern in MEP size over time for subject nr. 8. Periods of consecutive large MEPs (in green) are alternated with periods of small amplitudes (in red). Overall, the mean length of time periods of consecutive equal responses (TP-ER) was significantly longer in subjects than in surrogates $(p=0.05)$ and the number of TP-ER was significantly lower $(p=0.04)$. The top row of Figure 5.2A shows 

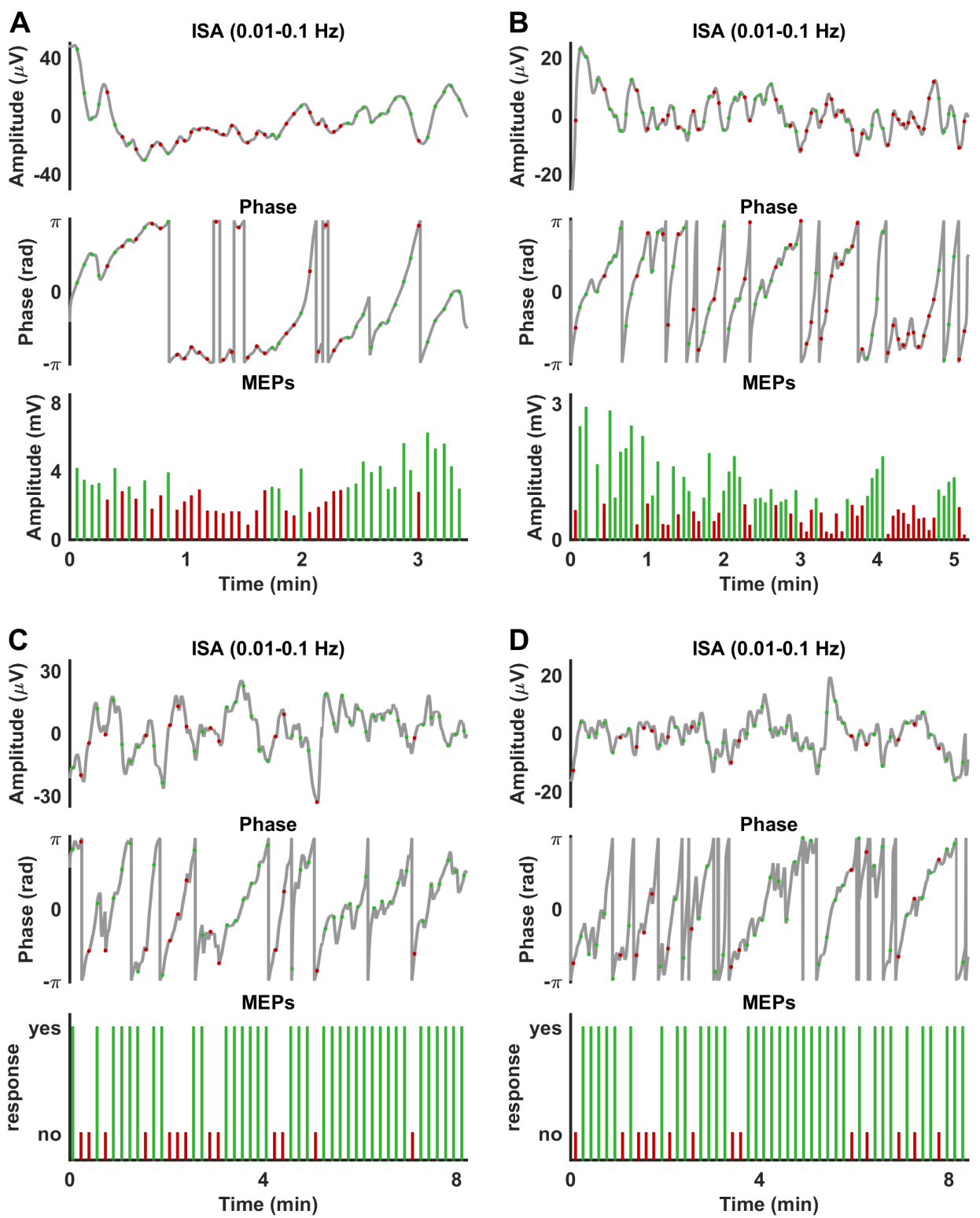

Figure 5.1: Four examples of infraslow activity (ISA; top row), the corresponding phase (middle row) and MEPs (bottom row) in subjects stimulated at three intensities: A) $120 \%$ rMT (nr. 8), B) $110 \%$ rMT (nr. 19) and C-D) $100 \%$ rMT (nrs. 30 and 27). The moments of stimulation are represented by green and red dots (top and middle rows) or bars (bottom row). In green the 'large' and 'present' MEPs and in red the 'small' and 'absent' MEPs. In all subjects infraslow activity is present, with peak amplitudes larger than $5 \mu \mathrm{V}$. 
Table 5.2: Overview of parameter $\mathrm{b}$ and correlation coefficient $R^{2}$ of fitted sine function through the linear (MEP amplitudes) circular (phase of infraslow activity) data of subjects stimulated at two intensities: $120 \%$ rMT (nrs. 1-13) and 110\% rMT (nrs. 14-23). For the 1000 surrogates either the $5^{\text {th }}$ and $95^{\text {th }}$ percentile (parameter b) or only the $95^{\text {th }}$ percentile $\left(R^{2}\right)$ is given.

\begin{tabular}{lccccccccccccc}
\hline \multicolumn{110}{c}{ Stimulation intensity $120 \%$ rMT } \\
\hline Subject & $\mathbf{1}$ & $\mathbf{2}$ & $\mathbf{3}$ & $\mathbf{4}$ & $\mathbf{5}$ & $\mathbf{6}$ & $\mathbf{7}$ & $\mathbf{8}$ & $\mathbf{9}$ & $\mathbf{1 0}$ & $\mathbf{1 1}$ & $\mathbf{1 2}$ & $\mathbf{1 3}$ \\
b & -0.38 & $0.25^{*}$ & 0.24 & -0.15 & $-0.26^{*}$ & 0.31 & -0.15 & $-0.91^{*}$ & $-0.19^{*}$ & $0.72^{*}$ & 0.22 & -0.54 & $0.06^{*}$ \\
b: $\mathbf{5}^{\text {th }}$ & -0.61 & -0.24 & -0.24 & -0.17 & -0.24 & -0.34 & -0.29 & -0.53 & -0.17 & -0.42 & -0.24 & -0.63 & -0.04 \\
b: $95^{\text {th }}$ & 0.67 & 0.25 & 0.27 & 0.20 & 0.26 & 0.39 & 0.31 & 0.57 & 0.19 & 0.47 & 0.26 & 0.63 & 0.05 \\
$\boldsymbol{R}^{2}$ & 0.04 & 0.10 & 0.07 & 0.06 & 0.09 & 0.07 & 0.03 & $0.26^{*}$ & 0.09 & $0.31^{*}$ & 0.07 & 0.09 & $0.13^{*}$ \\
$\boldsymbol{R}^{2}: 95^{\text {th }}$ & 0.13 & 0.12 & 0.12 & 0.11 & 0.12 & 0.12 & 0.12 & 0.12 & 0.10 & 0.11 & 0.11 & 0.11 & 0.12 \\
\hline
\end{tabular}

\section{Stimulation intensity $110 \% \mathrm{rMT}$}

\begin{tabular}{lcccccccccc}
\hline Subject & 14 & 15 & 16 & 17 & 18 & 19 & 20 & 21 & 22 & 23 \\
b & 0.16 & $0.97^{*}$ & 0.18 & $-0.21^{*}$ & -0.12 & $-0.24^{*}$ & 0.10 & -0.13 & $0.23^{*}$ & 0.24 \\
b: $5^{\text {th }}$ & -0.16 & -0.71 & -0.19 & -0.16 & -0.15 & -0.22 & -0.12 & -0.15 & -0.15 & -0.37 \\
b: $95^{\text {th }}$ & 0.18 & 0.80 & 0.20 & 0.17 & 0.16 & 0.23 & 0.13 & 0.17 & 0.15 & 0.42 \\
$\boldsymbol{R}^{2}$ & 0.06 & $0.12^{*}$ & 0.05 & $0.10^{*}$ & 0.03 & 0.07 & 0.04 & 0.04 & $0.14^{*}$ & 0.02 \\
$\boldsymbol{R}^{2}: 95^{\text {th }}$ & 0.07 & 0.08 & 0.08 & 0.08 & 0.08 & 0.08 & 0.08 & 0.07 & 0.08 & 0.07 \\
\hline
\end{tabular}

"significant values (parameter b: outside 5-95 percentile range surrogates; correlation coefficient $R^{2}$ : larger than $95^{\text {th }}$ percentile surrogates)

the probability of TP-ER as a function of their length. Even though a length of 1 has the highest probability, also periods of 5, 9 or 12 consecutive repeats are seen. As the probabilities of these longer TP-ER fall outside the 5-95 percentile range of surrogates, temporal clustering is present in this subject. Of all subjects, $69 \%$ showed significant temporal clustering.

In a similar way, Figure 5.3A shows the pattern in MEP size over phase for the same subject, as well as the probability of PP-ER as a function of their length. Overall, the mean length of PP-ER was not significantly longer in subjects than in surrogates $(p=0.08)$ and the number of PP-ER was not significantly lower $(p=0.11)$. Of all subjects, $69 \%$ showed significant phase clustering.

Two examples of the relation between MEP amplitude and phase of infraslow activity are presented in Figure 5.4A-B. In both subjects MEP amplitude significantly depended on the phase, since parameter $b$ fell outside the 5-95 percentile range of the surrogates, see Table 5.2. Significant phase dependence was found in $46 \%$ of the subjects. In half of these subjects ( $23 \%$ of all subjects) we found a weak but significant correlation, represented by $R^{2}>95^{\text {th }}$ percentile of surrogates, see Table 5.2. 

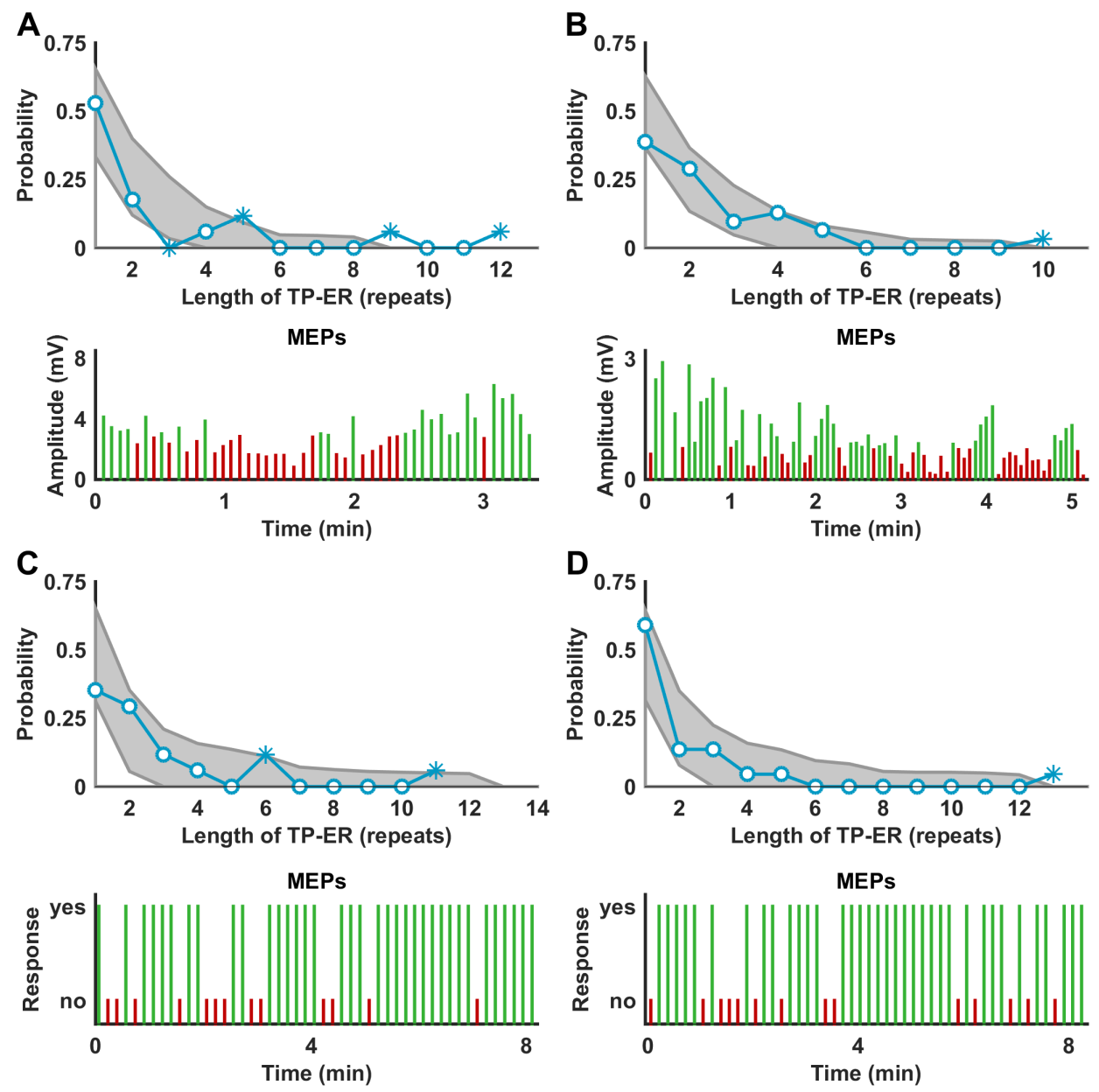

Figure 5.2: Four examples of significant temporal clustering in subjects stimulated at three intensities: A) $120 \%$ rMT (nr. 8), B) $110 \%$ rMT (nr. 19) and C-D) $100 \%$ rMT (nrs. 30 and 27). The top row shows the probability curve of time periods of consecutive equal responses (TP-ER) as a function of their length (in blue), including the 5-95 percentile range of the surrogate data (in grey). The asterisk represents significant probability values that fall outside the 5-95 percentile range of surrogates. The bottom row shows the pattern in MEP size (A-B) or occurrence (C-D) over time. In green the 'large' and 'present' MEPs and in red the 'small' and 'absent' MEPs. 

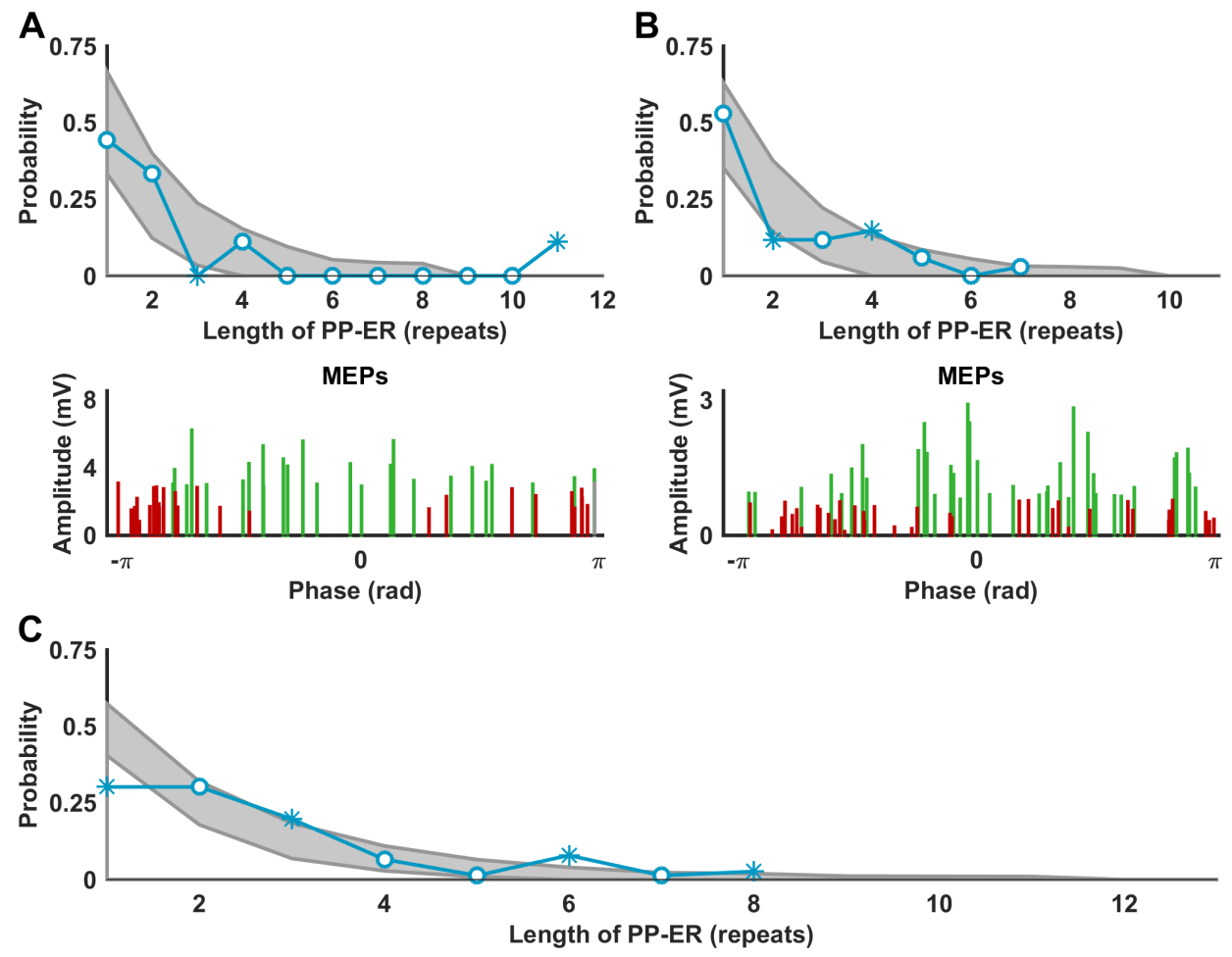

MEPs

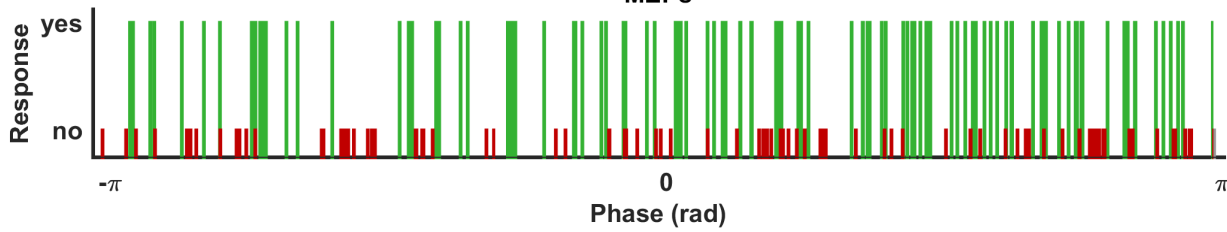

Figure 5.3: Three examples of significant phase clustering in subjects stimulated at three intensities: A) $120 \%$ rMT (nr. 8), B) $110 \%$ rMT (nr. 19) and C) $100 \%$ rMT (nr. 30). The top row shows the probability curve of phase periods of consecutive equal responses (PP-ER) as a function of their length (in blue), including the 5-95 percentile range of the surrogate data (in grey). The asterisk represents significant probability values that fall outside the 5-95 percentile range of surrogates. The bottom row shows the pattern in MEP size (A-B) or occurrence (C) over phase. In green the 'large' and 'present' MEPs and in red the 'small' and 'absent' MEPs. Please note that the phase is periodic, so that amplitudes around $\pi$ (in grey) are also presented around $-\pi$, forming a longer PP-ER. 

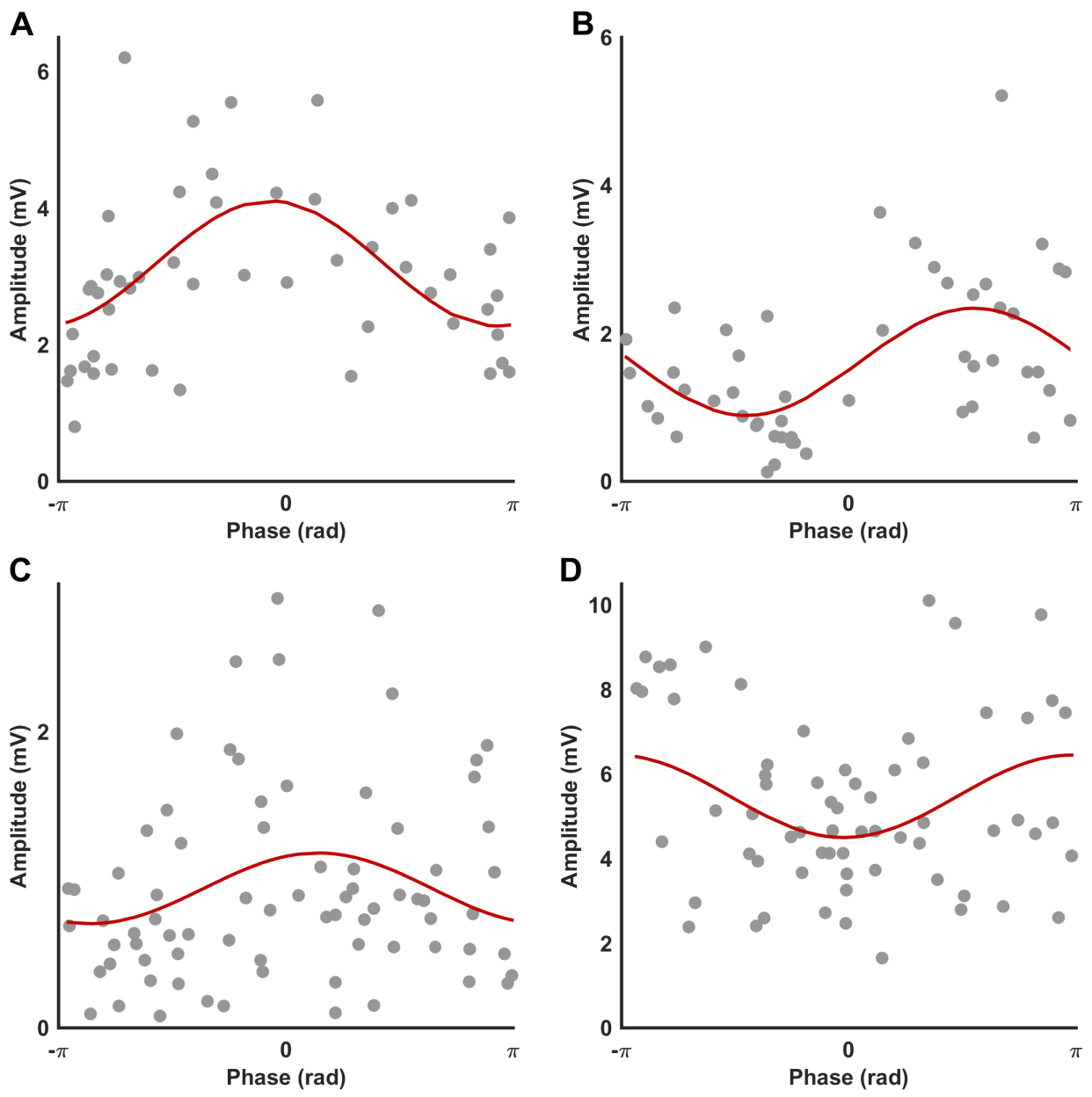

Figure 5.4: Four examples of significant dependence of MEP amplitude on the phase of the infraslow activity in subjects stimulated at two intensities: A-B) $120 \%$ rMT (nrs. 8 and 10) and C-D) $110 \%$ rMT (nrs. 19 and 15). The least-square method was used to fit a sine function (red line) through the MEP amplitudes and their corresponding phases (grey dots). 


\subsubsection{Stimulation intensity $110 \%$ rMT}

The mean length of TP-ER was significantly longer in subjects than in surrogates $(p=0.003)$ and the number of TP-ER was significantly lower $(p<0.001)$. Significant temporal clustering was found in $70 \%$ of the subjects, see Figure $5.2 \mathrm{~B}$ for an example.

We found significant phase clustering in $60 \%$ of the subjects, see Figure 5.3B for an example. However, the mean length of PP-ER was not significantly longer in subjects than in surrogates $(p=0.45)$ and the number of PP-ER was not significantly lower $(p=0.48)$.

Figure 5.4C-D shows the relation between MEP amplitude and phase of the infraslow activity for two subjects. Overall, we found a significant phase dependence in $40 \%$ of the subjects and a significant phase correlation in $30 \%$ of the subjects, see Table 5.2.

\subsubsection{Stimulation intensity $100 \%$ rMT}

The mean length of TP-ER was significantly longer in subjects than in surrogates $(p=0.001)$ and the number of TP-ER was significantly lower $(p<0.001)$. All subjects $(100 \%)$ showed significant temporal clustering in at least two out of four blocks of 50 pulses. Clusters were formed by periods of consecutive present MEPs (in green) or absent MEPs (in red), see Figure 5.2C-D. In most subjects the stimulation intensity was only slightly adjusted between blocks, due to some drift in rMT, see Table 5.1.

Of all subjects, 80\% showed significant phase clustering, see Figure 5.3C. Furthermore, the mean length of PP-ER was not significantly longer in subjects than in surrogates $(p=0.35)$ and the number of PP-ER was not significantly lower $(p=0.33)$.

\subsection{Discussion}

In this study we explored whether infraslow activity modulates cortical excitability, by evaluating the presence of temporal and phase clustering of TMS evoked responses. We found significant temporal clustering in all subjects stimulated at the rMT and in most of the subjects stimulated above rMT (69\% for $120 \%$ rMT and $70 \%$ for $110 \%$ rMT). Furthermore, for all three stimulation intensities the mean length of TP-ER was significantly longer in subjects than in surrogates and the number of TP-ER was significantly lower. Temporal clustering of MEP size and MEP occurrence suggests underlying determinism of cortical excitability instead of randomly generated fluctuations.

When evaluating MEP size, significant phase clustering was present in $60-69 \%$ of the subjects, while this was $80 \%$ when evaluating the occurrence of a MEP. No significant differences were found in mean length and number of PP-ER. In $40-46 \%$ of the subjects we found a significant dependence of MEP amplitude on the phase of the infraslow 
activity. In addition, we found a weak $\left(R^{2}<0.31\right)$ but significant amplitude-phase correlation in $23-30 \%$ of the subjects. We assumed a sinusoidal relation between amplitude and phase, due to the periodic nature of the phase. The fitted sinus curves had no constant shape as can be seen in Figure 5.4, while Bergmann et al. (2012) reported that MEP amplitudes were consistently larger when evoked during the rising state (phase between $-\pi$ and 0 rad) compared to the falling state (phase between 0 and $\pi \mathrm{rad}$ ) of the slow oscillation. This discrepancy might be explained by the fact that we measured subjects during wakefulness instead of during NREM sleep, where MEP responses do not resemble wake-like responses. In addition, we applied a relatively small number of pulses over the entire range between $-\pi$ and $\pi$ and the phase at the moment of stimulation was determined offline. In contrast, Bergmann et al. (2012) used an automatic real time detection algorithm to trigger TMS pulses very specifically during either the rising or falling state. This targeted approach makes it possible to discriminate two conditions (rising and falling state), each containing a higher number of trials.

The presence of both temporal and phase clustering was more prominent for MEP occurrence than for MEP amplitude. The fact that a stronger association was found for MEP occurrence, might be related to differences in methodology and statistics. A smaller number of pulses were given to evaluate the relation between infraslow activity and MEP amplitude compared to MEP occurrence, 50-75 versus 200 single pulses, respectively. Furthermore, the large/small distribution was always $50 / 50 \%$ for the MEP amplitude, while the present/absent distribution could be unbalanced for MEP occurrence. The influence of both factors on the variation in surrogate data might explain our finding.

Reduction of variability has been explored using brain-state triggered TMS, as applied by Bergmann et al. (2012). In this type of closed-loop systems, the TMS pulse is applied as a function of the simultaneously recorded instantaneous EEG (Zrenner et al., 2016). Recently this approach was applied by Zrenner et al. (2018) to synchronize TMS pulses to the phase of ongoing mu-oscillations in the alpha band for the sensorimotor cortex. Stimulation during the positive peak of mu-oscillations (low-excitability state) resulted in significantly smaller MEP amplitudes, than during the negative peak (high-excitability state). Furthermore, a long-term potentiation (LTP)-like increase in excitability was seen for repetitive stimulation $(100 \mathrm{~Hz})$ during the high-excitability state only. While no change in excitability was observed when targeting the lowexcitability state or random phases of the mu-oscillations (Zrenner et al., 2018). This indicates that the therapeutic potential of TMS could be optimized by targeting a specific oscillatory phase, due to a reduction of the TMS response variability. 


\subsubsection{Generation and relevance of infraslow activity}

Various mechanisms have been proposed to be involved in the generation of lowfrequency fluctuations. Aladjalova showed that infraslow activity can be recorded in cortical slabs without any subcortical input (Aladjalova, 1964), suggesting that cortical neurons have an intrinsic ability to generate very slow activity. Infraslow oscillations have also been recorded in the thalamus in rats (Albrecht et al., 1998; Albrecht and Gabriel, 1994), in anesthetized guinea pigs in vivo (He, 2003) and in individual thalamocortical neurons in slices of cat sensory thalamic nuclei (Hughes et al., 2011; Lörincz et al., 2009; Parri et al., 2001; Parri and Crunelli, 2001).

Further, infraslow activity may result from glia cells or the blood brain barrier (Amzica and Steriade, 2000; Vanhatalo et al., 2004; Voipio et al., 2003). In addition, nonbrain contributions to infraslow electrical activity recorded from the scalp may result from respiration or changes in skin resistance. Depending on the relative power of these other sources of infraslow activity, correlations between our readouts for cortical excitability may be modified, which may explain the variability in the phasedependency that we found for the MEP amplitude.

The biological significance of infraslow activity in relation to cortical excitability is not fully clear (Berger et al., 2014; Iscan et al., 2016; Zrenner et al., 2018). Most likely, however, the functional role of infraslow brain rhythms is similar to what has been reported for brain rhythms in general: to realize local-global interactions between neuronal networks and preservation of persistent activity (Buzsáki and Draguhn, 2004). For example, beta frequencies appear to be involved in synchronization of remote networks in the brain, while gamma rhythms are used for relatively local computations (Buzsáki and Watson, 2012; Gonzalez et al., 2014; Kopell et al., 2000). Infraslow EEG fluctuations are correlated with resting state network dynamics (Grooms et al., 2017; Hiltunen et al., 2014), suggesting a role in synchronizing network activity across large areas across the neocortex. As such synchrony involves variations in excitability of the involved networks (Pesaran et al., 2018), responses to external perturbations as applied in our study will vary, as well.

\subsubsection{Limitations}

We only explored whether infraslow activity modulates cortical excitability, instead of evaluating multiple frequency bands. Significant associations between the phase of brain oscillations and the MEP amplitude have been reported for rhythms in the alpha, beta and gamma bands (Berger et al., 2014; Mäki and IImoniemi, 2010a; Monto et al., 2008; Zrenner et al., 2018). However, the large TMS artifact hampers offline estimation of the phase at the moment of stimulation. Linear interpolation over a period of $25 \mathrm{~ms}$ 
around the TMS pulse did not influence the infraslow activity filtered between 0.01 $0.1 \mathrm{~Hz}$, while it did affect faster frequency bands. Further, EEG signals are typically not strictly periodic. Therefore, extracting and interpretation of the physical meaning of phase from such signals is not straightforward (Kraskov et al., 2004). However, other studies used a similar approach to exact phase from the EEG and observed phasedependencies as well, ranging from perception of touch (Monto et al., 2008) and selectivity of neuronal firing ( $\mathrm{Ng}$ et al., 2013) to LTP (Zrenner et al., 2018).

Another limitation is the potential contribution of sweat that can cause voltage fluctuations in the infraslow frequency range. However, since all subjects were measured at rest, it is unlikely that subjects sweated a lot during the TMS session. Further, if sweat artifacts would have been present, correlations with our readouts are improbable.

Temporal clustering could be influenced by changes in coil positioning or physiological factors, such as respiration rate, blood pressure or attention level (Conte et al., 2007; Mars et al., 2007). In most subjects, however, the rMT did not vary much between the four blocks of 50 pulses that were applied to study the relation between infraslow activity and MEP occurrence. In case of drift, the stimulation intensity was slightly adjusted to provoke that MEPs were only evoked $50 \%$ of the time. Although, changing the intensity might influenced the infraslow activity and cortical excitability, we tried to obtain a present/absent MEP distribution that was as close to $50 / 50 \%$ as possible.

We evaluated infraslow activity measured at the electrode closest to the stimulation location. This choice was made because others found modulatory effects that were topographically delimited to the stimulated motor cortex. No significant effects for the MEP amplitude were found for oscillations measured in the contralateral motor cortex, or in the occipital or frontal cortex (Bergmann et al., 2012; Mäki and Ilmoniemi, 2010a; Sauseng et al., 2009). For the amplitude of TMS evoked potential (TEP) components, however, modulatory effects were not necessarily restricted to the stimulation site. During NREM sleep, TEP amplitudes were significantly larger when evoked during the rising state of slow oscillations $(<1 \mathrm{~Hz})$, compared to the falling state. Although this effect was still most prominent at the stimulation location for the $\mathrm{P} 40$ and N120 peaks, fronto-central and centro-parietal electrodes showed the largest differences for the late N400 peak (Bergmann et al., 2012). It thus seems to depend on the TMS outcome measure whether the modulatory effect is strictly local or global.

\subsection{Conclusion}

Significant temporal clustering was found in all subjects stimulated at the rMT and in most of the subjects stimulated above rMT. In addition, the majority of subjects showed significant phase clustering for MEP occurrence and MEP size. Furthermore, in 
approximately a quarter to half of the subjects we found a significant correlation and dependence of MEP amplitude on the phase of infraslow activity, respectively. The presence of both temporal and phase clustering, as well as phase dependence makes the contribution of infraslow activity as a modulator of cortical excitability likely, even though additional mechanisms are probably involved as well.

\section{Acknowledgements}

We thank all the subjects for their participation. Furthermore, we would like to thank Esther ter Braack for providing the data measured in subjects stimulated at $110 \%$ rMT and Carin Eertman for her assistance during the TMS measurements.

\section{Funding}

This study was financed by the Dutch TWIN foundation for neuromodulation. The funding source played no role in the design of the study, collection, analysis and interpretation of the data, and writing of the manuscript.

\section{Disclosures}

M.J.A.M. van Putten is co-founder of Clinical Science Systems, Leiden, the Netherlands. 




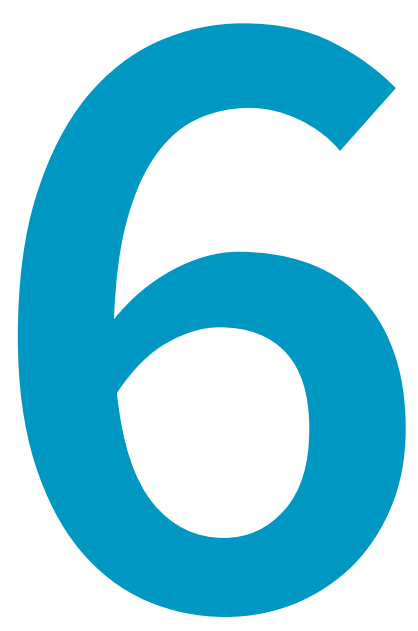

\section{Single and paired pulse transcranial magnetic stimulation in drug naïve epilepsy}

A.A. de Goede, E.M. ter Braack and M.J.A.M. van Putten

Clinical Neurophysiology 2016;127(9):3140-3155 


\section{Abstract}

Objective: Transcranial magnetic stimulation (TMS) measures cortical excitability and is therefore potentially suitable as an additional tool for epilepsy diagnostics and therapy evaluation. In this review we discuss the application of TMS in epilepsy research and systematically analyze single and paired pulse TMS outcomes from 31 drug naïve patient studies.

Results: Despite a large variety in used TMS protocols, there was no relation between specific protocol aspects and the occurrence of significant results. Protocols were often not in accordance with latest guidelines and recommendations.

Cortical excitability, as measured by TMS, was increased in drug naïve epilepsy patients, being most prominent for generalized epilepsy. Single pulse TMS indicated a trend towards a lower resting motor threshold and a prolonged cortical silent period for generalized epilepsy, while inconclusive results were found for focal epilepsy. The paired pulse TMS outcomes, short intracortical inhibition $(\mathrm{SICI})$ and long intracortical inhibition ( $\mathrm{LICl}$ ), showed the most consistent significant increase in cortical excitability in generalized and focal epilepsy patients.

Conclusion: Future epilepsy research should especially focus on the interstimulus intervals 2 and $5 \mathrm{~ms}$ for $\mathrm{SICl}$ and 250 and $300 \mathrm{~ms}$ for $\mathrm{LICl}$. Furthermore, combining TMS with electroencephalography may contribute to analysis on an individual patient level. Highlights:

- TMS reveals an increased cortical excitability in drug naïve epilepsy patients.

- $\mathrm{SICl}$ and $\mathrm{LICl}$ consistently showed significant differences between epilepsy patients and controls.

- TMS protocols were often not in accordance with reported guidelines. 


\subsection{Introduction}

Epilepsy is characterized by an enduring predisposition to generate epileptic seizures (Fisher et al., 2005, 2014), due to a cortical imbalance between excitatory and inhibitory activity resulting in an increased cortical excitability (McCormick and Contreras, 2001; McNamara, 1994). The electroencephalogram (EEG) is routinely used for epilepsy diagnostics and seizure classification (Berg et al., 2010; King et al., 1998; Krumholz et al., 2007). However, a major limitation of the routine EEG is the moderate sensitivity in epilepsy (King et al., 1998; Krumholz et al., 2007), which has motivated the search for additional techniques to assess cortical excitability. In addition, once the diagnosis of epilepsy is confirmed, only the absence or recurrence of epileptic seizures provides an indication as to whether anti-epileptic drugs (AEDs) have any effects (Engel, 2008; Kimiskidis et al., 2014). Assessing cortical excitability shortly after starting AEDs may provide earlier information on therapy success.

Transcranial magnetic stimulation (TMS) was first described in 1985 by Barker and colleagues as a non-invasive and easy to use technique to directly stimulate the human motor cortex (Barker et al., 1985). Although TMS can be uncomfortable at high stimulation intensities, discomfort was largely reduced compared to electrical stimulation. Over the past three decades, TMS has evolved from a relatively simple method to activate corticospinal motor pathways (Barker et al., 1985) to a sophisticated technique to assess cortical excitability (Kujirai et al., 1993; Valls-Solé et al., 1992), connectivity (Fox et al., 1997; Ilmoniemi et al., 1997; Paus et al., 1997) and plasticity (Liepert et al., 1995; Pascual-Leone and Torres, 1993; Stefan et al., 2000) in a wide variety of neuropsychiatric conditions. Recent review papers discussed the ability of TMS as a technique to get more insight into the pathophysiology of epilepsy, as well as a tool for epilepsy diagnostics and therapy evaluation (Badawy et al., 2014a; Bauer et al., 2014; Kimiskidis et al., 2014). Although hyperexcitable cortical networks were frequently reported in epilepsy patients, results were not always consistent, which currently limits the clinical application of TMS (Badawy et al., 2014a; Bauer et al., 2014). Three possible factors are most likely responsible for inconsistent findings in epilepsy research: 1) recruitment of drug-treated patients, 2) methodological differences between studies and 3) poor correlation of TMS to clinical variables (Badawy et al., 2014a).

In this review we describe the application of single and paired pulse TMS paradigms and their corresponding measures of cortical excitability in epilepsy. In order to avoid the confounding effects of AED treatment, we systematically analyze TMS outcomes in drug naïve epilepsy patients only. In addition, we discuss a number of methodological aspects that may have influenced these findings. 


\subsection{Basic principles of the TMS technique}

TMS is based on the fundamental principles of electromagnetic induction. The very strong pulse of electric current in the stimulation coil produces a magnetic field which is oriented perpendicular to the coil. If placed on the head of the subject, this timevarying magnetic field induces electrical currents in the cortex, which if large enough, depolarize neurons and initiate action potentials (Barker et al., 1985; Hallett, 2000; Rothwell, 1997). The effectiveness of TMS is based on the ability of the magnetic field to pass through the high-resistance skull. As the magnetic field attenuates rapidly with increasing distance from the coil (Hess et al., 1987; Roth et al., 1991) stimulation is limited to the superficial cortical layers (Heller and van Hulsteyn, 1992). Although in theory any superficial cortical brain area can be stimulated, the most commonly used stimulation site is still the primary motor cortex due to its characteristic signature output. Other stimulation sites include the visual cortex (Amassian et al., 1989b), the language areas (Pascual-Leone et al., 1991), the sensorimotor cortex (Seyal et al., 1992), the (dorsolateral) prefrontal cortex (George et al., 1995; Pascual-Leone and Hallett, 1994) and the association cortex (Seyal et al., 1995). Action potentials are generated by sufficiently large transmembrane currents activating the voltage-gated sodium channels. These transmembrane currents will be generated if the magnetically induced ionic current has a spatial gradient parallel with the orientation of the axon (Kobayashi and Pascual-Leone, 2003; Maccabee et al., 1993). This essentially follows from Kirchhoff's law: to preserve charge, an increase or reduction in the ionic current inside the axon must be met by a compensatory transmembrane current. Activation also occurs at positions where the axons bend out of the induced electric field (Kobayashi and Pascual-Leone, 2003). The size of the stimulated brain area depends on the TMS coil that is used. TMS is usually applied with a circular or figure-of-eight coil, although new coil designs are being explored (Deng et al., 2008, 2013; Roth et al., 2002). The maximum value of the magnetic field is located directly under the center of the coil. Figure-ofeight coils result in a more focal stimulation point compared to circular coils (Cohen et al., 1990; Jalinous, 1991; Rösler et al., 1989). The TMS pulse type can be monophasic, with only the first phase of the pulse inducing an effect in the cortex, or biphasic, where especially the second phase of the pulse is responsible for activating the cortex. Biphasic pulses have a longer duration than monophasic pulses and therefore start to activate the motor cortex at a lower intensity, resulting in a lower motor threshold (Kammer et al., 2001; Maccabee et al., 1998; Niehaus et al., 2000; Sommer et al., 2006).

\subsubsection{Simulation paradigms}

Stimulation may be applied using a single pulse at a time in the single pulse TMS paradigm (Barker et al., 1985), using pairs of consecutive stimuli separated by a 
variable interval in the paired pulse TMS paradigm (Kujirai et al., 1993; Valls-Solé et al., 1992) or using trains of multiple pulses at specific frequencies in the repetitive TMS paradigm (Pascual-Leone et al., 1994). Single and paired pulse TMS assess the functional state of the cortex and can detect changes in cortical excitability over time (Kobayashi and Pascual-Leone, 2003). Due to our focus on the use of TMS as a tool for epilepsy diagnostics and therapy evaluation, this review paper will only cover the single and paired pulse TMS paradigms. In contrast, repetitive TMS can induce temporary or permanent changes in excitability (Chen et al., 1997; Pascual-Leone et al., 1994; Ziemann et al., 1998). This makes that this paradigm is currently investigated for therapeutic purposes in epilepsy (Hsu et al., 2011; Kimiskidis, 2010; Kimiskidis et al., 2014; Lefaucheur et al., 2014).

\subsubsection{TMS combined with electromyography}

Traditionally, a single or paired TMS pulse is applied to the motor cortex and the corresponding motor evoked potential (MEP) is measured in a peripheral muscle on the contralateral side using electromyography (EMG) (Barker et al., 1985). The TMS pulse depolarizes neurons, initiating descending volleys of action potentials from the cerebral cortex along the spinal cord to peripheral nerves. Early corticospinal volleys (so called direct-waves or D-waves) are the result of direct excitation of corticospinal neurons, while later volleys (so called indirect-waves or I-waves) originate from indirect trans-synaptic excitation of corticospinal neurons (Day et al., 1987; Patton and Amassian, 1954). At threshold intensities, electrical stimulation evokes Dwaves, while magnetic stimulation preferentially generates I-waves (Day et al., 1987; Di Lazzaro et al., 1998a, 2001). For magnetic stimulation, D-waves are evoked at threshold intensities when the induced current is in the latero-medial direction, or at very high stimulation intensities for the posterior-anterior and anterior-posterior direction (Di Lazzaro et al., 1998a, 2001). These D- and/or I-waves initiate, if large enough, action potentials in spinal motor neurons. Propagation of these action potentials along peripheral motor axons eventually results in muscle contraction and generation of a MEP. This implies that the corresponding MEP is influenced by cortical as well as spinal excitability (Abbruzzese and Trompetto, 2002; Valls-Solé et al., 1992). Several characteristics of the EMG and MEP can be used to assess excitability: motor threshold (MT), MEP amplitude and cortical silent period (CSP) for the single pulse TMS paradigm and short intracortical inhibition (SICI), intracortical facilitation (ICF) and long intracortical inhibition (LICI) for the paired pulse TMS paradigm. These characteristics are summarized in Figure 6.1. 

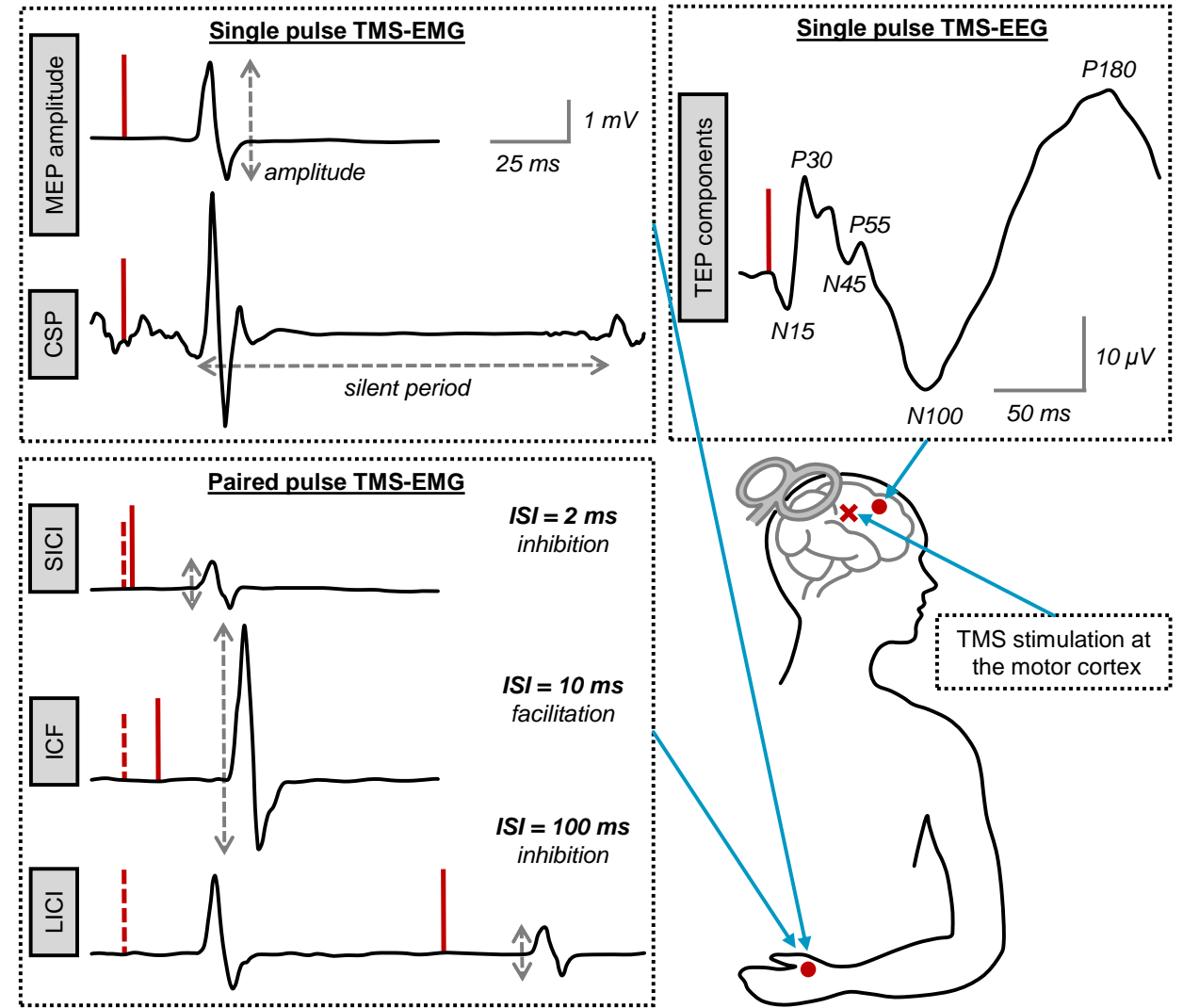

Figure 6.1: Outcome measures for TMS-EMG and TMS-EEG. Upper panels correspond to the single pulse TMS paradigm and the lower panel to the paired pulse TMS paradigm; red solid lines $=$ TMS pulse, red dashed lines = conditioning TMS pulse. $\mathrm{MEP}=$ motor evoked potential, CSP = cortical silent period, $\mathrm{TEP}=\mathrm{TMS}$ evoked potential, $\mathrm{SICI}=$ short intracortical inhibition, $\mathrm{ICF}=$ intracortical facilitation, $\mathrm{LICI}=$ long intracortical inhibition and ISI = interstimulus interval. 


\section{Motor threshold}

MT is defined as the minimum TMS pulse intensity needed to elicit reproducible MEPs in a target muscle. In case of a fully relaxed target muscle this threshold is called the resting motor threshold (rMT), while the active motor threshold (aMT) is measured using a slightly contracted muscle (Rossini et al., 1994, 2015); the aMT is lower compared to the rMT (Hess et al., 1987). MT reflects the integrated excitability of cortical, corticospinal and spinal motor neurons (Wassermann et al., 2008).

\section{MEP amplitude}

The MEP amplitude is measured from peak-to-peak, see Figure 6.1. It increases with stimulation intensity following a sigmoid curve (Devanne et al., 1997; Möller et al., 2009; Pitcher et al., 2003). The amplitude depends not only on the number of cortical motor neurons activated but also on the number of recruited motor neurons in the spinal cord, the number of motor neurons discharging more than once to the TMS pulse, synchronization of the TMS-induced motor neuron discharges and the contraction state of the target muscle (Wassermann et al., 2008). Due to spinal facilitation, MEP amplitude is higher in contracted muscles than in relaxed muscles (Abbruzzese and Trompetto, 2002; Hess et al., 1986, 1987; Wassermann et al., 2008).

\section{Cortical silent period}

When TMS is performed while the target muscle is slightly contracted, it causes an interruption of ongoing voluntary EMG activity for a certain time period following the MEP, see Figure 6.1. This period, which lasts a few hundred milliseconds at high stimulation intensities, is called the CSP and consists of two parts (Calancie et al., 1987; Fuhr et al., 1991; Inghilleri et al., 1993; Ziemann et al., 1993). Spinal gammaaminobutyric acid (GABA)-A receptor mediated inhibitory mechanisms are thought to contribute to the early part of the CSP up to the first 50-75 ms (Inghilleri et al., 1996; Kimiskidis et al., 2006), while long-lasting GABA-B receptor mediated inhibition from motor cortical origin contributes to the later part (Siebner et al., 1998; Stetkarova and Kofler, 2013). The duration of CSP is a measure of cortical inhibition and increases with stimulation intensity following a sigmoid curve (Inghilleri et al., 1993; Kimiskidis et al., 2005; Orth and Rothwell, 2004; Roick et al., 1993).

\section{Short intracortical inhibition}

During paired pulse TMS two consecutive pulses are applied with a variable interstimulus interval (ISI). SICI occurs when a sub-threshold conditioning TMS pulse is given 1-5 ms before a supra-threshold test pulse (Kujirai et al., 1993), see Figure 6.1. The hypothesis is that the conditioning pulse activates low-threshold cortical inhibitory mechanisms, thereby inhibiting the generation of action potentials by the test pulse, 
eventually leading to inhibition of the test MEP amplitude (Di Lazzaro et al., 1998b; Hanajima et al., 1998; Kujirai et al., 1993). SICI is thought to be related to GABA-A receptor mediated inhibition (Hanajima et al., 1998; Kujirai et al., 1993).

\section{Intracortical facilitation}

ICF can be obtained by increasing the interval between the sub-threshold conditioning pulse and supra-threshold test pulse to 6-30 ms (Kujirai et al., 1993; Ziemann et al., 1996b), see Figure 6.1. Facilitation of the test MEP amplitude is the net result of the balance between strong $N$-methyl-D-aspartate (NMDA) receptor mediated facilitation (Schwenkreis et al., 1999; Ziemann et al., 1998) and weaker GABA-A receptor mediated inhibition (Hanajima et al., 1998; Inghilleri et al., 1996).

\section{Long intracortical inhibition}

$\mathrm{LICl}$ occurs when a supra-threshold conditioning and test pulse are given 50-400 ms after each other. In this case, both the conditioning pulse and test pulse evoke a MEP. The first conditioning MEP response equals a single test response, while the second test MEP response is inhibited (Valls-Solé et al., 1992), see Figure 6.1. $\mathrm{LICl}$ is thought to be related to GABA-B receptor mediated inhibition (McDonnell et al., 2006; Pierantozzi et al., 2004; Werhahn et al., 1999).

\subsubsection{TMS combined with electroencephalography}

The effect of a single or paired TMS pulse applied to the cortex can also be assessed by measuring the direct neuronal response in the stimulated brain using TMS in combination with EEG (Ilmoniemi et al., 1997; Ilmoniemi and Kičić, 2010). Because assessment of cortical excitability no longer depends on a peripheral response, it is possible to stimulate and measure responses in other brain areas than the most commonly used primary motor cortex. The EEG response provoked by a TMS pulse is called the TMS evoked potential (TEP). The largest TEP amplitudes are measured directly under the TMS coil and diminish with increasing distance from the stimulated brain area (Ilmoniemi and Kičić, 2010; Komssi et al., 2002). The TEP has distinct characteristics when averaging multiple responses from the same stimulation location, consisting of the following components: N15 (negative deflection at approximately 15 ms after TMS pulse), P30 (positive deflection), N45, P55, N100 and P180 (Ilmoniemi and Kičić, 2010; Komssi et al., 2004; Paus et al., 2001), see Figure 6.1. Both the amplitudes and latencies of TEP components are measures of cortical excitability (Rogasch and Fitzgerald, 2013). The N45 is related to GABA-A receptor mediated inhibition, whereas the N100 is linked to GABA-B receptor mediated inhibition (Premoli et al., 2014a).

Because the intensity of the TMS pulse is very high, TMS-EEG is technically challenging. First of all, a dedicated amplifier and TMS compatible electrodes are necessary to 
measure EEG signals while applying TMS (Ilmoniemi and Kičić, 2010). The TMS pulse itself induces a large artifact, which can be taken out with a sample-and-hold amplifier or by zero-interpolation. The TMS pulse can also induce scalp muscle activity, leading to a second large bipolar artifact. This second artifact can be reduced by changing the coil position and angle (Mutanen et al., 2013), or by using artifact rejection methods such as independent component analysis (Hernandez-Pavon et al., 2012; Korhonen et al., 2011; Rogasch et al., 2014) or principal component analysis (ter Braack et al., 2013a). Another issue is the overlapping auditory evoked potential (AEP), as each TMS pulse is accompanied by a clicking sound. This AEP can be largely prevented by letting the subject wear headphones playing noise and placing a thin layer of foam between coil and head (ter Braack et al., 2013b; Massimini, 2005).

\subsection{TMS research in epilepsy}

Despite its potential to be an additional tool for epilepsy diagnostics and therapy evaluation, the clinical value of TMS is still limited (Badawy et al., 2014a; Bauer et al., 2014; Kimiskidis et al., 2014). Findings are not consistent, most likely due to differences in patient population and/or used methodology (Badawy et al., 2014a; Tassinari et al., 2003). The consistency of TMS outcomes can be increased by applying strict criteria regarding included subjects and research protocol (Tassinari et al., 2003). Before discussing TMS findings in epilepsy, we shortly describe three general aspects of the epilepsy research protocol that may affect the consistency of TMS outcome measures. Selection criteria for this review are based on these aspects.

\subsubsection{General aspects of epilepsy research protocol}

\section{Epilepsy classification and stimulation site}

Based on seizure type, patients can be classified as having either generalized or focal seizures (Berg et al., 2010; McCormick and Contreras, 2001; Roger et al., 1989). Usually both hemispheres are stimulated in epilepsy patients. However, in patients with generalized seizures, no interhemispheric differences in cortical excitability are observed in large patient cohorts (Badawy et al., 2007, 2013d; Lee et al., 2015). Therefore, stimulation of only the dominant hemisphere (according to handedness) should be sufficient in generalized epilepsy. In contrast, in patients with focal seizures both the 'affected' ipsilateral hemisphere containing the epileptic focus and the 'non-affected' contralateral hemisphere should be studied. In particular in drug naïve patients, it is common to find an increase in cortical excitability solely for the ipsilateral hemisphere, while values comparable to healthy subjects are measured on the contralateral side (Badawy et al., 2007, 2013e; Lee et al., 2015; Werhahn et al., 2000). Studies in patients with focal seizures showed that it is possible to detect changes 
in cortical excitability, even though the epileptic focus is distant from the stimulated primary motor cortex (Badawy et al., 2015; Cantello et al., 2000; Hamer, 2005; Werhahn et al., 2000). The primary motor cortex is the most commonly used stimulation site for TMS, because it is relatively easy to locate due to its characteristic and objective signature output. However, stimulating the epileptic focus or brain regions closely connected to the focus might reveal larger differences between epilepsy patients and healthy controls in the response to TMS.

\section{Anti-epileptic drugs}

AEDs can be categorized into three classes: 1) modulators of voltage-gated ion channels, 2) enhancers of synaptic inhibition and 3) inhibitors of synaptic excitation (Rogawski and Löscher, 2004). We refer to Ziemann et al. (2015) and Ziemann (2004) for a complete overview of the effects of drugs on cortical excitability. Indeed, a reversal of cortical hyperexcitability was found after starting AEDs in drug naïve patients (Badawy et al., 2010a, 2013a; Reutens et al., 1993). However, normalization of cortical excitability was only seen in patients who became seizure-free after chronic use of AEDs. This finding was independent of seizure characteristics or type of AED. In refractory patients excitability did not change and remained increased after AED treatment (Badawy et al., 2010a, 2013a).

\section{Control group}

To measure a difference in cortical excitability, it is possible to use epilepsy patients as their own control by measuring each patient multiple times under different conditions, for example before and after starting AEDs. However, it is more common to use a control group of healthy subjects without a history of epileptic seizures. Another option is to use relatives, for example asymptomatic siblings, as a control group. However, cortical excitability profiles in unaffected siblings can be similar to their affected siblings. Indications for hyperexcitability were found in siblings of patients with different types of generalized and focal epilepsy (Badawy et al., 2013b).

\subsubsection{Search strategy for TMS epilepsy literature}

A systematic PubMed search (January 2016) using the search and MeSH terms "transcranial magnetic stimulation" or "TMS" and "epilepsy" identified 518 articles. Only full text original research articles written in English describing single and paired pulse TMS outcomes in a group of more than five epilepsy patients were selected. Five more articles were added after checking the reference lists, leaving a preliminary selection of 83 articles measuring single and/or paired pulse TMS in epilepsy patients. In order to minimize confounders in the epilepsy research protocol, we selected a final subset of articles comparing TMS outcomes between drug naïve epilepsy 
patients and healthy controls that were not described elsewhere. In addition, outcomes for the dominant hemisphere (when possible) for generalized epilepsy are presented separately from outcomes for both the 'affected' ipsilateral and 'nonaffected' contralateral hemispheres (when possible) for focal epilepsy. Patients were considered to be drug naive if they had never taken AEDs before, if they discontinued all AEDs at least one week before the TMS session or if AED serum levels were negative. The final selection of drug naïve studies consisted of 31 articles, see Figure 6.2, which were all TMS-EMG studies.

\subsubsection{TMS combined with electromyography}

\section{Motor threshold}

Of the 31 drug naïve studies, 25 articles compared the rMT between drug naïve epilepsy patients and healthy controls (Badawy et al., 2006, 2007, 2009, 2012, 2013c,d,e, 2014b, 2015; Badawy and Jackson, 2012; Brodtmann et al., 1999; Delvaux et al., 2001; Groppa et al., 2008; Kazis et al., 2006; Klimpe et al., 2009; Lee et al., 2015; Macdonell et al., 2001; Michelucci et al., 1996; Nezu et al., 1997; Puri et al., 2013; Reutens et al., 1993; Reutens and Berkovic, 1992; Suppa et al., 2009; Varrasi et al., 2004; Werhahn et al., 2000), see Figure 6.3. For generalized epilepsy, the majority of articles (13 out of 19) found a lower rMT in patients than in healthy controls (Badawy et al., 2007, 2009, 2012, 2013c,d, 2014b; Badawy and Jackson, 2012; Brodtmann et al., 1999; Kazis et al., 2006; Klimpe et al., 2009; Reutens et al., 1993; Reutens and Berkovic, 1992; Suppa et al., 2009), which corresponds to an increased cortical excitability in epilepsy. However, this increase in excitability in epilepsy was only significant in five studies (Badawy et al., 2013d; Kazis et al., 2006; Reutens et al., 1993; Reutens and Berkovic, 1992; Suppa et al., 2009). Badawy et al. (2013d) only found a significantly lower rMT in juvenile myoclonic epilepsy (JME), but not in juvenile absence epilepsy (JAE) and generalized epilepsy with tonic-clonic seizures (GE-TCS). In addition, a meta-analysis by Brigo et al. (2012) reported a non-significant trend towards lower rMT values for generalized epilepsy in general, but a significant decrease for JME.

Although hyperexcitability is most often seen in generalized epilepsy, six studies found a non-significant reduction in excitability (Badawy et al., 2006; Delvaux et al., 2001; Groppa et al., 2008; Lee et al., 2015; Macdonell et al., 2001; Puri et al., 2013). A possible explanation for an increase in rMT could be the activation of interictal compensatory mechanisms to prevent the spreading and/or recurrence of new seizures (Badawy et al., 2008; Delvaux et al., 2001; Lee et al., 2015), although it is not clear why these protective mechanisms may have influenced rMT only in these six studies. In two of the six studies, the decrease in excitability may be due to AEDs effects that were still present in patients who abstained from AEDs for more than one week (Macdonell et al., 2001) or one 


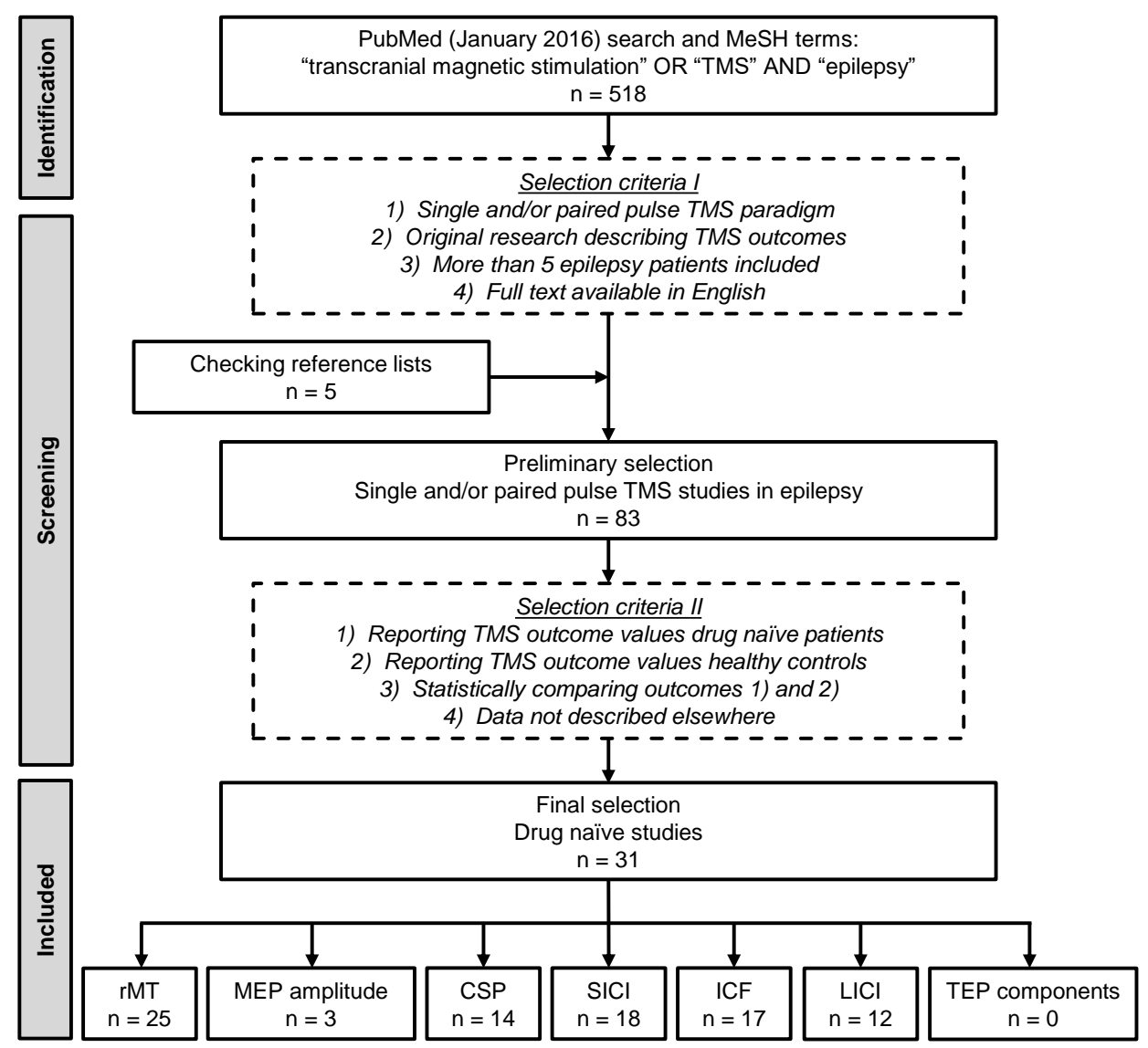

Figure 6.2: Search strategy in PubMed. A final subset of 31 drug naïve studies describing single and/or paired pulse TMS studies in epilepsy were selected for analysis. The following TMS outcomes were compared between epilepsy patients and healthy controls: $r M T=$ resting motor threshold, $\mathrm{MEP}=$ motor evoked potential, $\mathrm{CSP}=$ cortical silent period, $\mathrm{SICl}=$ short intracortical inhibition, ICF = intracortical facilitation, $\mathrm{LICl}=$ long intracortical inhibition and TEP = TMS evoked potential. The included 31 drug naïve studies only described TMS-EMG outcomes. 


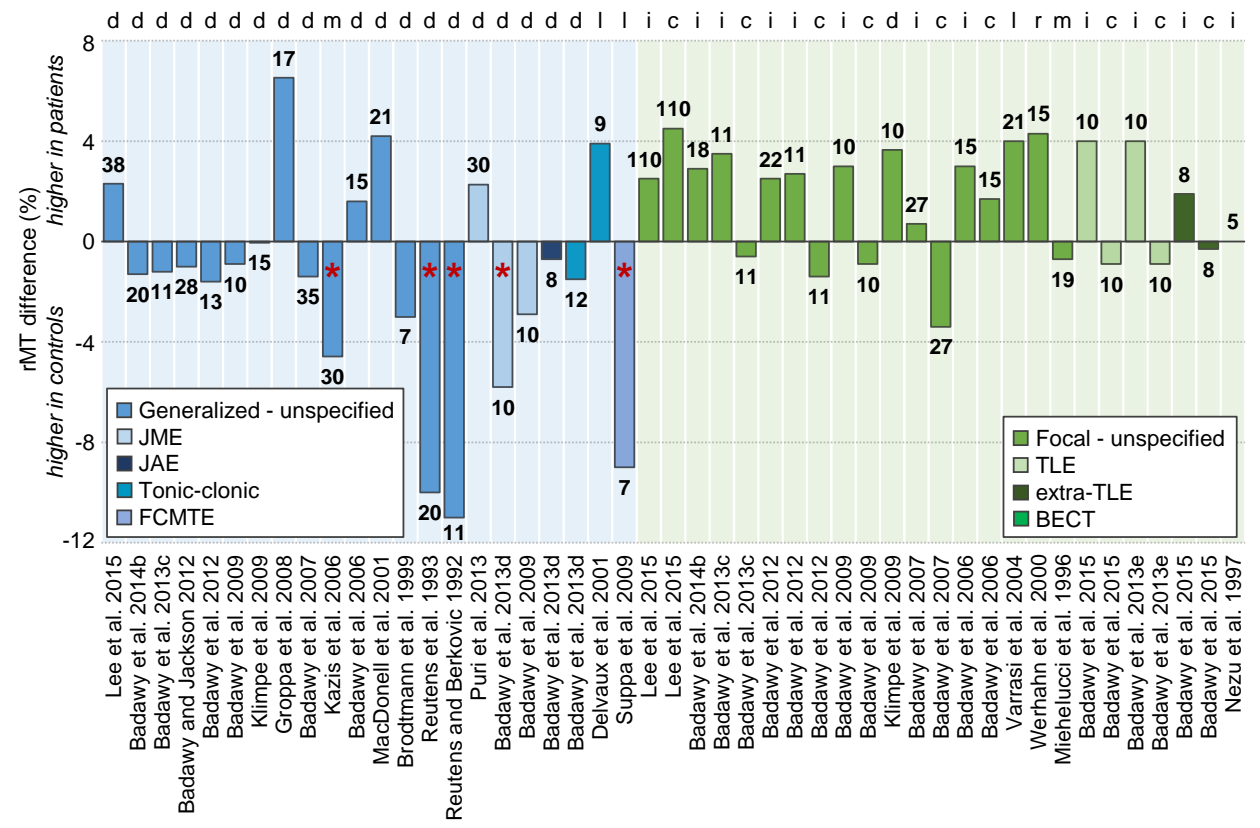

Figure 6.3: Difference in resting motor threshold (rMT) between drug naïve epilepsy patients and healthy controls. Generalized epilepsies are shown in blue and focal epilepsies are shown in green. The number of included epilepsy patients is noted above/below the corresponding bar and the stimulated hemisphere is stated above the plot; $d=$ dominant, $m=$ mean between both hemispheres, $\mathrm{l}=$ left, $\mathrm{r}=$ right, $\mathrm{i}=$ ipsilateral and $\mathrm{c}=$ contralateral. A red asterisk means that a statistical significant difference is reported between patients and controls. If studies reported more than one rMT, we selected the following value: rMT first session (Badawy et al., 2012), rMT second session (Delvaux et al., 2001), rested rMT (Badawy et al., 2006), morning rMT (Badawy et al., 2009; Puri et al., 2013) and postprandial rMT (Badawy et al., 2013c). JME = juvenile myoclonic epilepsy, JAE = juvenile absence epilepsy, FCMTE = familial cortical myoclonic tremor with epilepsy, TLE = temporal lobe epilepsy and BECT = benign childhood epilepsy with centrotemporal spikes. Overall, a trend towards hyperexcitability (lower rMT) is seen in generalized epilepsy. In focal epilepsy, rMT is higher for the ipsilateral than for the contralateral hemisphere. 
month (Lee et al., 2015). Chronic use of AEDs in epilepsy patients and healthy subjects leads to an increase in rMT (Ziemann et al., 2015), which decreases gradually following withdrawal of drugs (Hufnagel et al., 1990; Lee et al., 2005; Ziemann et al., 2015).

For focal epilepsy, none out of fifteen studies found a significant difference between patients and controls (Badawy et al., 2006, 2007, 2009, 2012, 2013c,e, 2014b, 2015; Badawy and Jackson, 2012; Klimpe et al., 2009; Lee et al., 2015; Michelucci et al., 1996; Nezu et al., 1997; Varrasi et al., 2004; Werhahn et al., 2000). This finding suggests that the value of rMT to detect increased excitability in drug naïve focal epilepsy patients is limited. Most studies (7 out of 8 ) measuring both hemispheres found a higher rMT on the ipsilateral side than on the contralateral side, which was significant in four studies (Badawy et al., 2007, 2012, 2013e, 2015). This phenomenon seems to be characteristic for new-onset focal epilepsy, as no interhemispheric differences are found anymore for chronic focal epilepsy (Badawy et al., 2015).

Most studies defined rMT as the minimum intensity needed to induce a MEP (peakto-peak amplitude $\geq 100 \mu \mathrm{V}$ ) in the abductor pollicis brevis (APB) muscle in $50 \%$ out of 10 trials. However, variations in MEP peak-to-peak amplitude, target muscle and percentage of MEPs out of number of trials were reported, see Table 6.1.

Although the APB was most commonly used (14 out of 25), the current recommendation is to use the first dorsal interosseous (FDI) muscle, because it is small, localized and easy to identify by means of palpation (Groppa et al., 2012).

Regarding the percentage of MEPs out of number of trials, Awiszus (2012) showed via a mathematical simulation that MT accuracy was only $47.6 \%$ when defining $\geq 5$ positive MEPs out of 10 trials, which increased to $96.2 \%$ for $\geq 10$ MEPs out of 20 trials. Based on this finding, current guidelines recommend to use 10 out of 20 trials to produce reproducible TMS outcomes (Rossini et al., 2015).

Accurate MT determination is of major importance as the stimulation intensity used during the TMS session is adjusted to each subject's individual MT. Although the relative frequency method is rather time consuming and requires a large number of stimuli (Groppa et al., 2012; Rossini et al., 2015), it is still the most commonly used method (23 out of 25) for MT estimation. Alternative methods include the adaptive method (Qi et al., 2011), the two-thresholds method (Mills and Nithi, 1997) and supervised parametric estimation (Tranulis et al., 2006). The adaptive method provides the most accurate and fastest MT estimation due to the small number of stimuli required (Awiszus, 2011; Qi et al., 2011) and is therefore recommended (Groppa et al., 2012; Rossini et al., 2015). In short, the adaptive method tries to estimate the probability of eliciting a MEP by TMS at a certain stimulation intensity. For each next pulse the intensity is selected that yields a $50 \%$ probability of evoking a MEP (Qi et al., 2011). 


\section{MEP amplitude}

Only three articles compared the MEP amplitude between drug naïve epilepsy patients and healthy controls (Klimpe et al., 2009; Lee et al., 2015; Silbert et al., 2015). None of these studies found significant differences between controls and either generalized (Klimpe et al., 2009; Lee et al., 2015; Silbert et al., 2015) or focal epilepsy patients (Klimpe et al., 2009; Lee et al., 2015). Due to the large intrinsic variability in amplitude to consecutive pulses given at the same intensity (Amassian et al., 1989a; Goldsworthy et al., 2016; Hess et al., 1987; Kiers et al., 1993; Roy Choudhury et al., 2011), MEP amplitude is not often used as a measure of cortical excitability (Cavaleri et al., 2015; Goldsworthy et al., 2016). The fact that all three studies used 10 or less pulses, where 20-30 pulses are indicated for reliable estimation of corticospinal excitability (Chang et al., 2016; Goldsworthy et al., 2016), might contribute to the limited value of MEP amplitude. A planned systematic study will provide more insight into the minimum number of pulses needed for reliable assessment of cortical excitability (Cavaleri et al., 2015).

Recent TMS-EEG studies suggest that the MEP amplitude is related to the state of ongoing EEG phase and power oscillations (Bergmann et al., 2012; Ferreri et al., 2014; Keil et al., 2014). Maybe the variability in MEP amplitude has more meaning than the amplitude itself, as the intertrial variability can provide important information concerning the state-dependency of corticospinal excitability (Ferreri et al., 2014). In addition, it may be relevant to focus on the MEP phases and the occurrence of polyphasic oscillations, as recently an increase in MEP polyphasia was found in generalized epilepsy patients compared to healthy controls (Chowdhury et al., 2015).

\section{Cortical silent period}

Of the 31 drug naïve studies, fourteen compared the CSP between epilepsy patients and healthy controls (Badawy et al., 2007; Cantello et al., 2006; Delvaux et al., 2001; Ertas et al., 2000; Groppa et al., 2008; Joo et al., 2008; Kazis et al., 2006; Klimpe et al., 2009; Lee et al., 2015; Macdonell et al., 2001; Puri et al., 2013; Suppa et al., 2009; Varrasi et al., 2004; Werhahn et al., 2000), see Figure 6.4. For generalized epilepsy, most studies (6 out of 11) found a significant longer CSP in patients than in healthy controls (Cantello et al., 2006; Joo et al., 2008; Kazis et al., 2006; Lee et al., 2015; Macdonell et al., 2001; Puri et al., 2013). This finding is supported by a recent meta-analysis from Cincotta et al. (2015), where the prolongation of CSP in generalized epilepsy was related to hyperactivation of inhibitory circuits, acting as a protective mechanism to prevent recurrence of new seizures. Five studies found a shorter CSP in patients (Badawy et al., 2007; Delvaux et al., 2001; Groppa et al., 2008; Klimpe et al., 2009; Suppa et al., 2009). However, this decrease in CSP was only significant in one (Suppa et al., 2009). The fact that 
Table 6.1: Overview of protocol aspects regarding rMT determination. Differences in rMT between epilepsy patients and healthy controls were reported in 25 studies. The recommended option according to recent guidelines is underlined. Used TMS protocols in drug naïve epilepsy studies were often not in accordance with recent guidelines and recommendations.

\begin{tabular}{|c|c|c|c|}
\hline Protocol element & \#/total & Significance & References \\
\hline \multicolumn{4}{|c|}{ MEP peak-to-peak amplitude } \\
\hline \multirow[t]{2}{*}{$>50 \mu \mathrm{V}^{\mathrm{a}}$} & $7 / 25$ & Significant & Suppa et al. 2009 \\
\hline & & Not significant & $\begin{array}{l}\text { Klimpe et al. 2009; Lee et al. 2015; Michelucci et al. } \\
\text { 1996; Puri et al. 2013; Varrasi et al. 2004; Werhahn et al. } \\
2000\end{array}$ \\
\hline \multirow[t]{2}{*}{$>100 \mu \mathrm{V}$} & $17 / 25$ & Significant & $\begin{array}{l}\text { Badawy et al. 2013d; Reutens et al. 1993; } \\
\text { Reutens and Berkovic } 1992\end{array}$ \\
\hline & & Not significant & $\begin{array}{l}\text { Badawy et al. 2006, 2007, 2009, 2012, 2013c,e, 2014b, } \\
\text { 2015; Badawy and Jackson 2012; Brodtmann et al. } \\
\text { 1999; Delvaux et al. 2001; Groppa et al. 2008; Macdonell } \\
\text { et al. 2001; Nezu et al. } 1997\end{array}$ \\
\hline \multirow[t]{2}{*}{ Other } & $1 / 25$ & Significant & Kazis et al. 2006 \\
\hline & & Not significant & - \\
\hline \multicolumn{4}{|l|}{ Target muscle } \\
\hline \multirow[t]{2}{*}{$\frac{\text { First dorsal inter- }}{\text { osseous }\left(\text { FDI) }^{\mathrm{b}}\right.}$} & $8 / 25$ & Significant & Kazis et al. 2006; Suppa et al. 2009 \\
\hline & & Not significant & $\begin{array}{l}\text { Delvaux et al. 2001; Klimpe et al. 2009; Lee et al. 2015; } \\
\text { Nezu et al. 1997; Varrasi et al. 2004; Werhahn et al. } 2000\end{array}$ \\
\hline \multirow[t]{2}{*}{$\begin{array}{l}\text { Abductor digiti } \\
\text { minimi (ADM) }\end{array}$} & $3 / 25$ & Significant & Reutens et al. 1993; Reutens and Berkovic 1992 \\
\hline & & Not significant & Macdonell et al. 2001 \\
\hline \multirow[t]{2}{*}{$\begin{array}{l}\text { Abductor pollicis } \\
\text { brevis (APB) }\end{array}$} & $14 / 25$ & Significant & Badawy et al. 2013d \\
\hline & & Not significant & $\begin{array}{l}\text { Badawy et al. 2006, 2007, 2009, 2012, 2013c,e, 2014b, } \\
\text { 2015; Badawy and Jackson 2012; Brodtmann et al. } \\
\text { 1999; Groppa et al. 2008; Michelucci et al. 1996; Puri et } \\
\text { al. } 2013\end{array}$ \\
\hline
\end{tabular}

\begin{tabular}{|c|c|c|c|}
\hline \multicolumn{4}{|c|}{ Percentage of MEPs out of number of trials } \\
\hline \multirow[t]{2}{*}{$\begin{array}{l}50 \% \text { out of less } \\
\text { than } 10 \text { pulses }\end{array}$} & $7 / 25$ & Significant & Reutens et al. 1993; Reutens and Berkovic 1992 \\
\hline & & Not significant & $\begin{array}{l}\text { Brodtmann et al. 1999; Lee et al. 2015; Macdonell et al. } \\
\text { 2001; Michelucci et al. 1996; Nezu et al. } 1997\end{array}$ \\
\hline \multirow[t]{2}{*}{$\begin{array}{r}50 \% \text { out of } \\
10 \text { pulses }\end{array}$} & $14 / 25$ & Significant & Badawy et al. 2013d; Suppa et al. 2009 \\
\hline & & Not significant & $\begin{array}{l}\text { Badawy et al. 2006, 2007, 2009, 2012, 2013c,e, 2014b, } \\
\text { 2015; Badawy and Jackson 2012; Groppa et al. 2008; } \\
\text { Puri et al. 2013; Werhahn et al. } 2000\end{array}$ \\
\hline \multirow[t]{2}{*}{$\frac{50 \% \text { out of more }}{{\text { than } 10 \text { pulses }^{c}}}$} & $\underline{2 / 25}$ & Significant & - \\
\hline & & Not significant & Delvaux et al. 2001; Varrasi et al. 2004 \\
\hline \multirow[t]{2}{*}{ Other } & $2 / 25$ & Significant & Kazis et al. 2006 \\
\hline & & Not significant & Klimpe et al. 2009 \\
\hline
\end{tabular}


Table 6.1: Continued.

\begin{tabular}{|c|c|c|c|}
\hline \multicolumn{4}{|c|}{ Method for rMT determination } \\
\hline \multirow[t]{2}{*}{$\begin{array}{l}\text { Relative frequency } \\
\text { method }\end{array}$} & $23 / 25$ & Significant & $\begin{array}{l}\text { Badawy et al. 2013d; Reutens et al. 1993; Reutens and } \\
\text { Berkovic 1992; Suppa et al. } 2009\end{array}$ \\
\hline & & Not significant & $\begin{array}{l}\text { Badawy et al. 2015, 2014b, 2013c,e, 2012, 2009, 2007, } \\
\text { 2006; Badawy and Jackson 2012; Brodtmann et al. } \\
\text { 1999; Delvaux et al. 2001; Lee et al. 2015; Macdonell et } \\
\text { al. 2001; Michelucci et al. 1996; Nezu et al. 1997; Puri et } \\
\text { al. 2013; Varrasi et al. 2004; Werhahn et al. } 2000\end{array}$ \\
\hline \multirow[t]{2}{*}{$\begin{array}{l}\text { Two-thresholds } \\
\text { method }\end{array}$} & $1 / 25$ & Significant & Kazis et al. 2006 \\
\hline & & Not significant & - \\
\hline \multirow[t]{2}{*}{ Adaptive method $^{a}$} & $1 / 25$ & Significant & - \\
\hline & & Not significant & Klimpe et al. 2009 \\
\hline
\end{tabular}

this significant CSP shortening was found in a very specific patient group with familial cortical myoclonic tremor with epilepsy (FCMTE), may explain the discrepancy to the overall prolongation of CSP seen in generalized epilepsy.

For focal epilepsy, CSP changes are mixed: four studies found non-significant shortening of CSP compared to controls (Badawy et al., 2007; Cantello et al., 2006; Klimpe et al., 2009; Werhahn et al., 2000), while two articles reported CSP prolongation (Lee et al., 2015; Varrasi et al., 2004) which was significant for the ipsilateral hemisphere in one (Lee et al., 2015). In accordance, Cincotta et al. (2015) found a non-significant slight shortening of CSP, indicating the limited opportunities of CSP as a measure of cortical excitability in drug naïve focal epilepsy patients. The fact that Ertas et al. (2000) mainly included patients with generalized epilepsy (9 out of 10) is consistent with finding a significant prolongation of CSP.

Most studies measured CSP from the TMS artifact to reappearance of voluntary EMG activity in a mild to moderate ( $<50 \%$ of maximum) contracted muscle at stimulation intensities as a percentage of rMT. However, variations in CSP analysis method, target muscle contraction or stimulation intensity may have led to the mixed results found in especially focal epilepsy, see Table 6.2.

Although it is common to define the beginning of the MEP response (first turning point) as the start of CSP (Groppa et al., 2012; Rossini et al., 2015; Wassermann et al., 2008), this option was only applied in 4 out of 14 studies. Alternatives include the moment of applying the TMS pulse (6 out of 14) and the end of the MEP response (2 out of 14) (King et al., 2006; Wassermann et al., 2008). To estimate CSP duration from several 


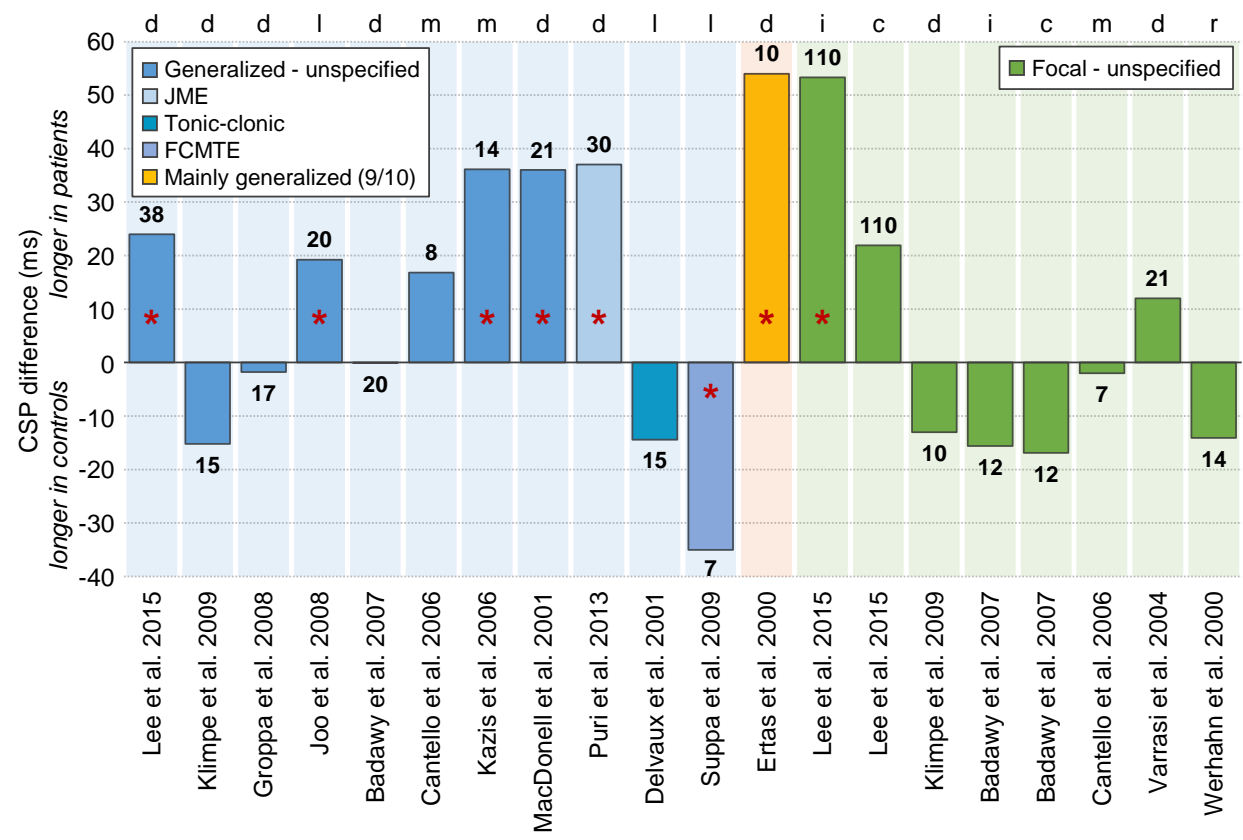

Figure 6.4: Difference in cortical silent period (CSP) duration between drug naïve epilepsy patients and healthy controls. Generalized epilepsies are shown in blue, focal epilepsies are shown in green and mixed studies are shown in yellow. The number of included epilepsy patients is noted above/below the corresponding bar and the stimulated hemisphere is stated above the plot; $\mathrm{d}=$ dominant, $\mathrm{m}=$ mean between both hemispheres, $\mathrm{l}=$ left, $\mathrm{r}=$ right, $\mathrm{i}=$ ipsilateral and $c=$ contralateral. A red asterisk means that a statistical significant difference is reported between patients and controls. If studies reported more than one stimulation intensity or more the one CSP, we selected the following value: CSP at intensity of 105\% rMT (Macdonell et al., 2001), CSP at intensity of 115\% rMT (Badawy et al., 2007), CSP at intensity of 150\% rMT (Joo et al., 2008; Lee et al., 2015), CSP at maximum stimulator output (Kazis et al., 2006), CSP values at intensity of $150 \%$ rMT from Cincotta et al. (2015) and patient values without photic stimulation (Groppa et al., 2008), CSP value controls from Cincotta et al. (2015), Cantello et al. (2006) and morning CSP (Puri et al., 2013). JME = juvenile myoclonic epilepsy and FCMTE = familial cortical myoclonic tremor with epilepsy. Overall, CSP prolongation is seen in generalized epilepsy, while CSP changes are mixed in focal epilepsy. 
Table 6.2: Overview of protocol aspects regarding CSP determination. Differences in CSP duration between epilepsy patients and healthy controls were reported in 14 studies. The recommended option according to recent guidelines is underlined. Used TMS protocols in drug naïve epilepsy studies were often not in accordance with recent guidelines and recommendations.

\begin{tabular}{|c|c|c|c|}
\hline Protocol element & \#/total & Significance & References \\
\hline \multicolumn{4}{|c|}{ CSP analysis method - determination of start of period } \\
\hline \multirow[t]{2}{*}{ MEP onset $^{\mathrm{a}}$} & $4 / 14$ & Significant & Joo et al. 2008; Kazis et al. 2006; Lee et al. 2015 \\
\hline & & Not significant & Groppa et al. 2008 \\
\hline \multirow[t]{2}{*}{ End MEP } & $2 / 14$ & Significant & Puri et al. 2013 \\
\hline & & Not significant & Delvaux et al. 2001 \\
\hline \multirow[t]{2}{*}{ TMS artifact } & $6 / 14$ & Significant & Macdonell et al. 2001 \\
\hline & & Not significant & $\begin{array}{l}\text { Badawy et al. 2007; Cantello et al. 2006; Klimpe et al. } \\
\text { 2009; Varrasi et al. 2004; Werhahn et al. } 2000\end{array}$ \\
\hline \multirow[t]{2}{*}{ Not described } & $2 / 14$ & Significant & Ertas et al. 2000; Suppa et al. 2009 \\
\hline & & Not significant & - \\
\hline \multicolumn{4}{|c|}{ Target muscle contraction } \\
\hline \multirow[t]{2}{*}{$\leq 50 \%{ }^{b}$} & $\underline{6 / 14}$ & Significant & Joo et al. 2008; Lee et al. 2015 \\
\hline & & Not significant & $\begin{array}{l}\text { Cantello et al. 2006; Groppa et al. 2008; Varrasi et al. } \\
\text { 2004; Werhahn et al. } 2000\end{array}$ \\
\hline \multirow[t]{2}{*}{$>50 \%$} & $2 / 14$ & Significant & Kazis et al. 2006 \\
\hline & & Not significant & Klimpe et al. 2009 \\
\hline \multirow[t]{2}{*}{ Maximal } & $5 / 14$ & Significant & Ertas et al. 2000; Macdonell et al. 2001; Puri et al. 2013 \\
\hline & & Not significant & Badawy et al. 2007; Delvaux et al. 2001 \\
\hline \multirow[t]{2}{*}{ Not described } & $1 / 14$ & Significant & Suppa et al. 2009 \\
\hline & & Not significant & - \\
\hline \multicolumn{4}{|l|}{ Stimulation intensity ${ }^{c}$} \\
\hline \multirow[t]{2}{*}{ Percentage of rMT } & $9 / 14$ & Significant & $\begin{array}{l}\text { Ertas et al. 2000; Joo et al. 2008; Lee et al. 2015; } \\
\text { Macdonell et al. } 2001\end{array}$ \\
\hline & & Not significant & $\begin{array}{l}\text { Badawy et al. 2007; Cantello et al. 2006; Groppa et al. } \\
\text { 2008; Varrasi et al. 2004; Werhahn et al. } 2000\end{array}$ \\
\hline \multirow[t]{2}{*}{ Percentage of aMT } & $2 / 14$ & Significant & - \\
\hline & & Not significant & Delvaux et al. 2001; Klimpe et al. 2009 \\
\hline \multirow[t]{2}{*}{$\begin{array}{l}\text { Maximum stimulator } \\
\text { output }\end{array}$} & $2 / 14$ & Significant & Kazis et al. 2006; Puri et al. 2013 \\
\hline & & Not significant & - \\
\hline \multirow[t]{2}{*}{ Not described } & $1 / 14$ & Significant & Suppa et al. 2009 \\
\hline & & Not significant & - \\
\hline
\end{tabular}

a Recommended by Groppa et al. (2012); Rossini et al. (2015).

${ }^{\mathrm{b}}$ Recommended by Rossini et al. (2015).

c No guidelines available. 
consecutive trials, the mean (or median) CSP duration based on single-trial recordings or the average over all rectified single trials can be calculated. The advantage of the latter method is that it provides a good visualization of the baseline EMG activity, making it easier to accurately define reappearance of voluntary activity (Groppa et al., 2012; Rossini et al., 2015). The end of CSP, either defined as the first reappearance of any EMG activity or as the return of EMG activity to levels measured prior to stimulation, can be difficult to establish (King et al., 2006; Wassermann et al., 2008). Semi-automatic methods, making it easier to determine the end of CSP reliably and objectively include the cumulative sum (Cusum) method (King et al., 2006), repeated Student's unpaired t-tests (Nilsson et al., 1997), the mean consecutive difference (Garvey et al., 2001) and a method based on filtering, squaring and threshold detection (Daskalakis et al., 2003). King et al. (2006) showed that under various conditions, the Cusum method consistently performed better than the other automated methods.

The amount of voluntary muscle activity or contraction level does not influence CSP duration (Haug et al., 1992; Inghilleri et al., 1993; Roick et al., 1993). Some authors advise to maintain sustained contraction at about $20 \%$ of maximal contraction (Rossini et al., 2015), while others recommend to use higher levels of contraction because this makes it easier to observe reappearance of EMG activity (Wassermann et al., 2008). To ensure a sustained level of contraction, acoustic and/or visual feedback of the EMG activity should be provided to the subject (Groppa et al., 2012; Rossini et al., 2015).

In epilepsy, stimulation intensity is commonly set as a percentage of rMT (9 out of 14) or aMT ( 2 out of 14). However, it is probably better to set the intensity to the CSP threshold, because MT and CSP may vary independently with AED use or disease state (Kimiskidis et al., 2005; Wassermann et al., 2008). However, guidelines prescribing which stimulation intensity should be applied are still lacking.

\section{Short intracortical inhibition and intracortical facilitation}

Eighteen articles measured SICI (Badawy et al., 2007, 2010a, 2012, 2013a,c,d,e, 2014b, 2015; Badawy and Jackson, 2012; Cantello et al., 2006; Delvaux et al., 2001; Joo et al., 2008; Klimpe et al., 2009; Lee et al., 2015; Silbert et al., 2015; Suppa et al., 2009; Werhahn et al., 2000) and seventeen ICF (Badawy et al., 2007, 2010a, 2012, 2013a,c,d,e, 2014b, 2015; Badawy and Jackson, 2012; Brodtmann et al., 1999; Cantello et al., 2006; Delvaux et al., 2001; Joo et al., 2008; Klimpe et al., 2009; Lee et al., 2015; Werhahn et al., 2000) in drug naïve epilepsy patients and healthy controls, see Figure 6.5. For generalized epilepsy, the most frequently investigated short ISIs are 2, 5, 10 and 15 ms. The majority of articles that included ISIs 2 and 5 ms found significant differences between generalized epilepsy patients and controls. Inhibition was found in healthy controls, while patients showed either facilitation (90\%) or reduced inhibition (10\%) 
(Badawy et al., 2007, 2010a, 2012, 2013a,c,d, 2014b; Badawy and Jackson, 2012; Silbert et al., 2015). Although ISIs 10 and 15 ms are usually included into the ICF protocol, these intervals never showed a significant difference between drug naïve patients and healthy controls (Badawy et al., 2007, 2010a, 2012, 2013a,c,d, 2014b; Badawy and Jackson, 2012; Joo et al., 2008; Lee et al., 2015). Therefore, the added value of these two ICF intervals is probably limited. When the average over several ICF intervals was taken, two studies reported a significant difference between patients and controls (Cantello et al., 2006; Delvaux et al., 2001), suggesting that individual ISIs may not be sensitive enough. Where Cantello et al. (2006) found an increase in facilitation in drug naïve idiopathic generalized epilepsy (IGE) patients, Delvaux et al. (2001) reported decreased facilitation in patients who experienced a first grand mal seizure in the last 48 hours. This discrepancy may be caused by differences in the time from last seizure (at least 3 weeks versus maximal 48 hours) and/or subject category (confirmed IGE versus first grand mal seizure).

For focal epilepsy, the short ISIs 2, 5, 10 and $15 \mathrm{~ms}$ are most frequently studied as well. Similar to generalized epilepsies most studies found significant differences between focal epilepsy patients and controls for SICl intervals 2 and 5 ms (Badawy et al., 2007, 2010a, 2012, 2013a,c,e, 2014b, 2015; Badawy and Jackson, 2012; Lee et al., 2015; Werhahn et al., 2000), but not for ICF intervals 10 and 15 ms (Badawy et al., 2007, 2010a, 2012, 2013a,e, 2014b, 2015; Badawy and Jackson, 2012; Lee et al., 2015). However, in focal epilepsy facilitation was restricted to the ipsilateral hemisphere, whereas inhibition was measured on the contralateral side just as in healthy subjects.

\section{Long intracortical inhibition}

Twelve articles measured $\mathrm{LICl}$ in drug naïve epilepsy patients and healthy controls (Badawy et al., 2007, 2010a, 2012, 2013a,c,d,e, 2014b, 2015; Badawy and Jackson, 2012; Brodtmann et al., 1999; Silbert et al., 2015), see Figure 6.6. For generalized epilepsy, the most frequently investigated long ISIs are 100, 150, 200, 250 and 300 ms. However, only for ISIs 150, 250 and 300 ms significant differences were found. Inhibition was found in healthy subjects, while facilitation was measured in patients (Badawy et al., 2007, 2010a, 2012, 2013a,c,d, 2014b; Badawy and Jackson, 2012; Brodtmann et al., 1999). Of the four studies evaluating ISI $50 \mathrm{~ms}$, three reported a significant increase in facilitation in epilepsy patients compared to controls (Badawy et al., 2010a, 2012; Badawy and Jackson, 2012).

For focal epilepsy, ISIs 250 and $300 \mathrm{~ms}$ are the only two intervals that were significantly different between patients and controls. Whereas inhibition was measured in healthy controls and in the contralateral hemisphere in focal epilepsy, facilitation was clearly present on the ipsilateral side (Badawy et al., 2007, 2010a, 2012, 2013a,c,e, 2014b, 2015; 

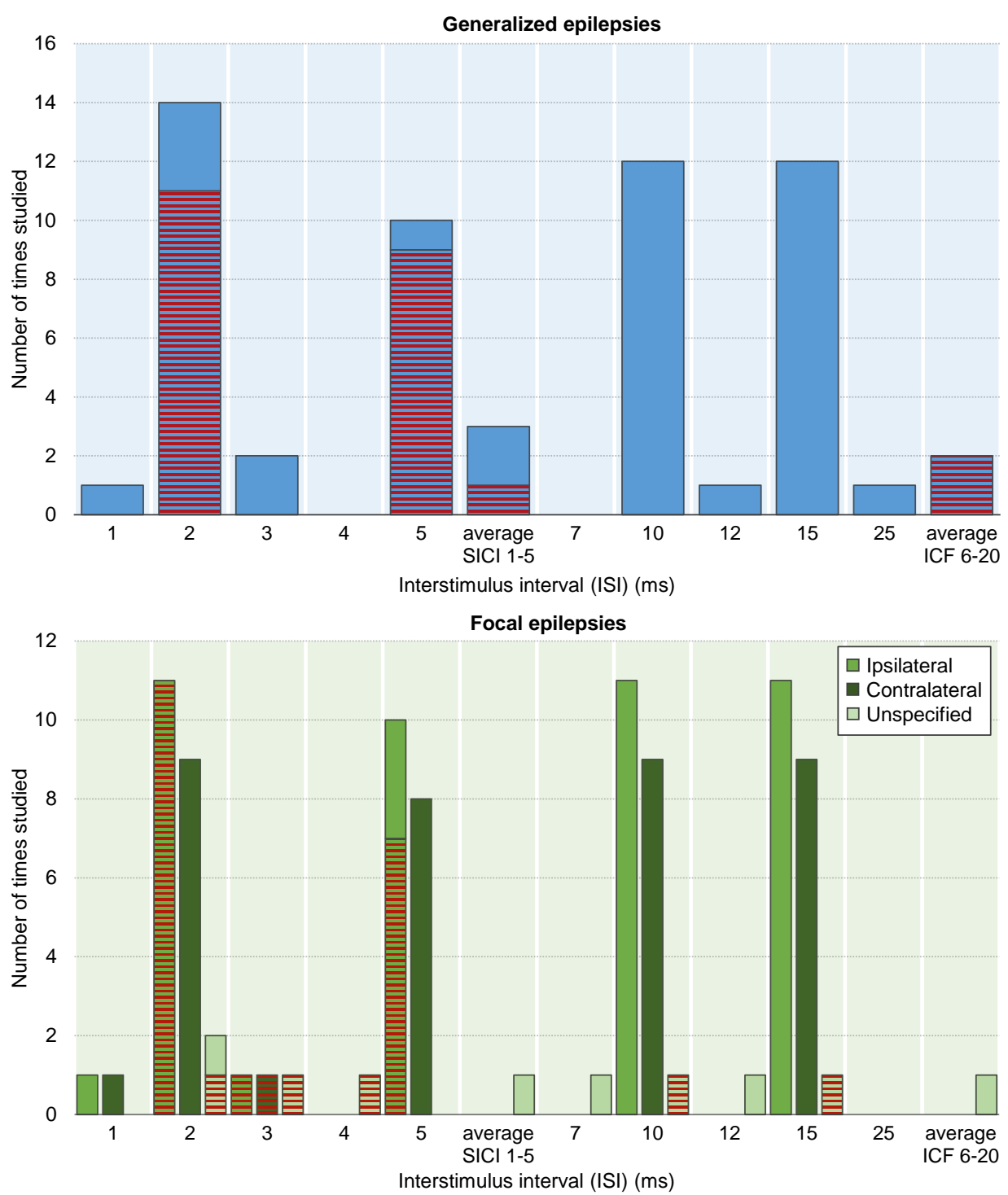

Figure 6.5: ISIs in drug naïve epilepsy patients and healthy controls for SICI and ICF. In blue (top panel) generalized epilepsies and in green (lower panel) focal epilepsies. On the y-axis the number of studies that measured an ISI for SICI/ICF is shown, the red stripes mean that a statistical significant difference was reported between patients and controls. If studies reported more than one value for $\mathrm{SICI}$ or ICF, we selected the following value: value at intensity $90 \%$ rMT for conditioning pulse (Klimpe et al., 2009), value at intensity $80 \%$ rMT for conditioning pulse (Silbert et al., 2015), SICl: average ISIs 3 and 4 ms; ICF: average ISIs 14 and 16 ms (Cantello et al., 2006), SICI: average ISIs 1, 2, 3, 4 and 5 ms; ICF: average ISIs 6, 7, 8, 9, 10, 15 and 20 ms (Delvaux et al., 2001) and postprandial values (Badawy et al., 2013c). Significant differences in generalized and focal epilepsy were mainly observed for ISIs 2 and $5 \mathrm{~ms}$. 

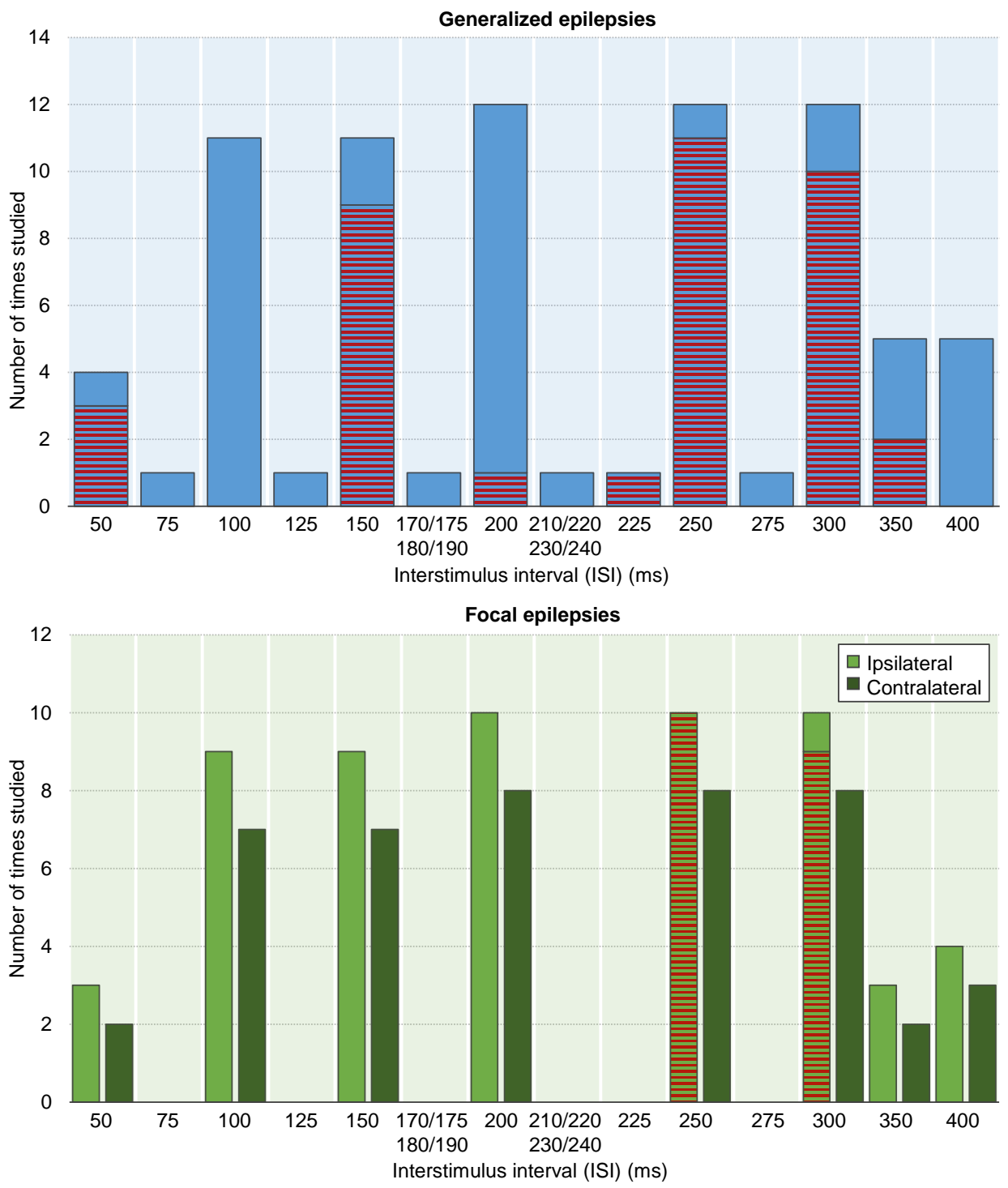

Figure 6.6: ISIs in drug naïve epilepsy patients and healthy controls for $\mathrm{LICl}$. In blue (top panel) generalized epilepsies and in green (lower panel) focal epilepsies. On the $y$-axis the number of studies that measured an ISI for $\mathrm{LICl}$ is shown, the red stripes mean that a statistical significant difference is reported between patients and controls. One study reported more than one value for $\mathrm{LICl}$, we selected the following value: postprandial LICI (Badawy et al., 2013c). Significant differences were observed for ISIs 50, 150, 250 and 300 ms for generalized epilepsies, while for focal epilepsies significant differences were only observed for ISIs 250 and 300 ms. 
Badawy and Jackson, 2012). In contrast to generalized epilepsy, ISIs 50 and $150 \mathrm{~ms}$ were never significant in focal epilepsy.

Both SICI and ICF protocols describe a sub-threshold conditioning TMS pulse followed by a supra-threshold test pulse (Kujirai et al., 1993), while for LICI both pulses are given at a supra-threshold stimulation intensity (Badawy et al., 2014a; Valls-Solé et al., 1992). Sub-threshold typically means $80 \%$ rMT or aMT and supra-threshold $120 \%$ rMT (Klimpe et al., 2009; Kujirai et al., 1993; Orth et al., 2003; Ortu et al., 2008). In general, the amount of inhibition and facilitation critically depends on the intensity of the conditioning pulse as well as the test pulse (Wassermann et al., 2008). Regarding the intensity of the conditioning pulse, it is of importance to use conditioning intensities around 8090\% rMT to obtain maximum SICI and ICF (Klimpe et al., 2009; Kujirai et al., 1993), especially for generalized epilepsy (Klimpe et al., 2009). In case of much lower or higher intensities it is even possible to measure a complete opposite effect; facilitation instead of inhibition and vice versa (Klimpe et al., 2009; Kujirai et al., 1993). Varying the intensity of the test pulse reveals that $\mathrm{SICl}$ increases with increasing stimulation intensity, while the strongest ICF and $\mathrm{LICl}$ are measured at low stimulation intensities (Sanger et al., 2001).

\subsection{General discussion}

Overall, TMS measures indeed showed an increased cortical excitability in drug naïve epilepsy patients, being most evident for the paired pulse TMS paradigm. This indicates that despite a diversity in pathways initiating epileptic seizures, there seems to be a common pathway in the cellular mechanisms underlying the expression of these seizures leading to a change in excitability (McCormick and Contreras, 2001). However, differences were only reported for patient groups and not at the individual patient level.

The single pulse TMS paradigm suggested increased cortical excitability along with enhanced inhibitory mechanisms for generalized epilepsy, as reflected by a trend towards a decreased rMT and a prolonged CSP. Hyperactivation of inhibitory circuits is thought to act as a protective mechanism to prevent seizure recurrence (Cincotta et al., 2015). However, inconclusive findings for rMT, MEP amplitude and CSP indicated the limited applicability of single pulse TMS outcomes in focal epilepsy patients.

Paired pulse TMS outcomes $\mathrm{SICl}$ and $\mathrm{LICl}$ showed the most consistent findings of increased excitability for both focal and generalized epilepsy. Therefore, paired pulse TMS seems to be more sensitive to detect changes in cortical excitability than single pulse TMS. Future paired pulse TMS research in epilepsy should particularly focus on the short ISIs 2 and $5 \mathrm{~ms}$ and the long ISIs 250 and $300 \mathrm{~ms}$. For generalized epilepsy the long ISIs 50 and $150 \mathrm{~ms}$ could also be included into the TMS protocol. 
TMS protocols used in epilepsy research showed a large variety in used parameters and were often not in accordance with latest guidelines and recommendations. We could not find any relation between specific protocol aspects and the occurrence of particular outcomes. General protocol aspects that could have influenced the results include coil type (circular or figure-of-eight), direction of the induced current (posterior-anterior or anterior-posterior), pulse type (mono- or biphasic), precision level during MT estimation (steps of $1 \%$ or $5 \%$ ), handedness, number of applied pulses, time between consecutive pulses, use of neuronavigation and prescriptions given to participants (maintain regular sleep, avoid alcohol/caffeine/nicotine and/or sessions on same time of the day and moment in menstrual cycle (only for females)). As many of these general aspects were not or not clearly described, it was not possible to indicate whether these aspects led to heterogeneity of outcomes and to which extent outcomes are comparable. Future studies should report methodological aspects more detailed to improve the quality of data collection and reporting, for example using a specific checklist (Chipchase et al., 2012).

\subsubsection{Limitations}

We only selected studies in drug naïve epilepsy patients, to remove the confounding effects of AEDs. Since we selected drug naïve patient studies, it is likely that most of the reported results were measured in new-onset epilepsy patients. Our conclusions can therefore not be extrapolated to epilepsy patients using AEDs or to chronic epilepsy patients without medication. In chronic epilepsy patients, the initial protective and compensatory mechanisms that are activated to prevent spreading and/or recurrence of new seizures are stabilized and excitability may differ from new-onset epilepsy patients (Badawy et al., 2007, 2015).

In this review we made a distinction between patients with generalized and focal epilepsy. As most studies described very heterogeneous patient cohorts, it was not possible to make a further subdivision into specific subtypes of epilepsy. It may be relevant to study more homogeneous patient cohorts, as despite some common pathways, specific subtypes may have a unique cortical excitability profile. An example are photosensitive patients with IGE where the phosphene threshold, measured at the primary visual cortex, might be a proper marker for excitability (Brigo et al., 2013). In these patients the decrease in phosphene threshold, the high phosphene prevalence and defective inhibition of the visual system suggests regional hyperexcitability of the primary visual cortex (Brigo et al., 2013; Strigaro et al., 2012). Furthermore, a hyperactive functional connection between the primary visual and motor cortex was found, when excitability of the primary visual cortex was studied in combination with the primary motor cortex (Strigaro et al., 2015). 
Although the findings of paired pulse TMS-EMG studies are the most consistent and promising, it should be noted that for $\mathrm{SICl} 10$ out of 18 studies and for $\mathrm{LICl} 10$ out of 12 studies were from Badawy and colleagues (Badawy et al., 2007, 2010a, 2012, 2013a,c,d,e, 2014b, 2015; Badawy and Jackson, 2012). Even though they have confirmed their own findings multiple times for different types of epilepsy, confirmatory studies from other research groups are needed. We think that reproducing these results is essential to appreciate the potential of TMS as a clinical tool in epilepsy.

\subsection{Future perspectives}

\subsubsection{TMS for diagnostics}

For TMS to be applicable as an additional tool for epilepsy diagnostics, it should detect a significant increased cortical excitability in new-onset epilepsy on an individual level. Ideally, TMS also discriminates between new-onset epilepsy and isolated seizures without recurrence, to aid in the decision whether or not to start treatment (Engel, 2008). Patients with isolated seizures also showed an increased excitability at ISIs 250 and $300 \mathrm{~ms}$, but facilitation was not as prominent as in epilepsy patients (Badawy et al., 2014b). At this moment, significant differences between new-onset epilepsy patients and healthy controls have only been reported on a group level. Potential diagnostic markers for focal and generalized epilepsy are $\mathrm{SICl}$ (ISIs 2 and $5 \mathrm{~ms}$ ) and $\mathrm{LICl}$ (ISIs 250 and $300 \mathrm{~ms}$ ), with additional measures being available for generalized epilepsy (CSP and $\mathrm{LICl}$ at ISIs 50 and $150 \mathrm{~ms}$ ). It appears to be more difficult to discriminate focal epilepsy patients from healthy subjects, than it is for generalized epilepsy patients.

\subsubsection{TMS for therapy evaluation}

Treatment success is determined by seizure reduction after starting AEDs (Engel, 2008; Kimiskidis et al., 2014). TMS could potentially aid in evaluating the response to AEDs in an early stage. AEDs have shown to affect cortical excitability even after a single dose, for example by increasing the rMT (Ziemann et al., 2015). For TMS to be applicable as a tool to evaluate therapy, the change in excitability after prescribing AEDs should differ between responders (well-controlled) and non-responders (poor-controlled), but not necessarily between epilepsy patients and healthy controls. Therefore, TMS measures suitable for therapy evaluation may differ from those for diagnosing epilepsy. Badawy et al. (2010a, 2013a) showed that at 8-12 weeks after starting AEDs, changes in rMT, $\mathrm{SICI}$ (ISIs 2 and $5 \mathrm{~ms}$ ) and $\mathrm{LICl}$ (ISIs 50, 150, 250 and $300 \mathrm{~ms}$ ) could discriminate between well-controlled and poor-controlled patients. Other studies also found differences in excitability after AED treatment, but only evaluated well-controlled patients (Cantello et al., 2006; Joo et al., 2008, 2010; Kazis et al., 2006; Manganotti et al., 
1999) Tataroglu et al. (2004). For the single pulse TMS paradigm, changes in rMT were found already after 15 days of AED use in focal epilepsy patients (Manganotti et al., 1999), suggesting that early therapy evaluation may indeed be possible.

\subsubsection{TMS combined with electroencephalography}

At present, no TMS-EEG studies have been published in drug naïve epilepsy patients. However, it would be interesting to combine TMS-EMG with EEG to try to move towards the level of an individual epilepsy patient. Especially the characteristic TEP components N45 and N100 are candidate markers, as they have recently been linked to GABA-A and GABA-B receptor mediated inhibition (Premoli et al., 2014a), similarly as for $\mathrm{SICl}$ and $\mathrm{LICl}$ (Ziemann et al., 2015). We are currently applying this multimodal approach of paired pulse TMS-EMG-EEG in patients presenting with a first seizure, where the diagnosis is still uncertain and in generalized and focal epilepsy patients before and after taking AEDs (trial ID: NL49901.044.14 and NL49854.044.14). Furthermore, Valentin et al. (2008) showed it is possible to induce a late EEG phenomena, resembling epileptiform discharges, with single pulse TMS in focal epilepsy patients but not in healthy controls. This TMS-EEG response could improve the diagnostic process and assist in identifying the epileptogenic zone (Kimiskidis, 2016; Valentin et al., 2008).

\section{Funding}

This work was financed by the Dutch TWIN Foundation for neuromodulation. The funding source played no role in the design of the study, collection, analysis and interpretation of the data, and writing of the manuscript. 



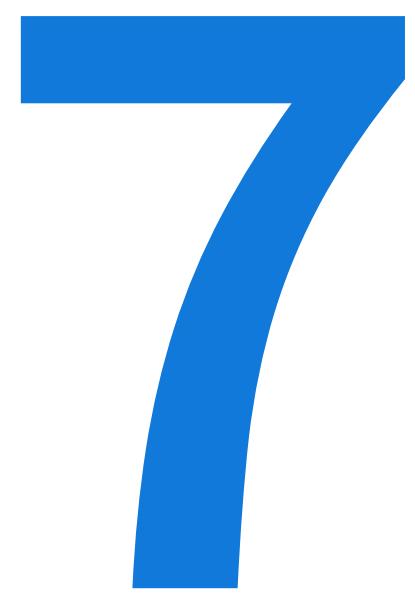

\section{Long-interval intracortical inhibition as biomarker for \\ epilepsy: a transcranial magnetic stimulation study}

P.R. Bauer*, A.A. de Goede*, W.M. Stern", A.D. Pawley", F.A. Chowdhury", R.M. Helling", R. Bouet, S.N. Kalitzin, G.H. Visser, S.M. Sisodiya, J.C. Rothwell, M.P. Richardson, M.J.A.M. van Putten and J.W. Sander *,\# These authors contributed equally to this work 


\section{Abstract}

Objective: Cortical excitability, as measured by transcranial magnetic stimulation (TMS) combined with electromyography (EMG), is a potential biomarker for the diagnosis and follow-up of epilepsy.

Methods: We report on long-interval intracortical inhibition (LICI) data measured in four different centers in healthy controls $(n=95)$, subjects with refractory genetic generalized epilepsy $(n=40)$ and with refractory focal epilepsy $(n=69)$. LICl was measured by applying two supra-threshold stimuli with an interstimulus interval (ISI) of 50,100,150, 200 and $250 \mathrm{~ms}$ and calculating the ratio between the response to the second (test stimulus) and to the first (conditioning stimulus). In all subjects, the median response ratio showed inhibition at all ISIs. Using a mixed linear-effects model, we compared the $\mathrm{LICI}$ response ratios between the different subject types. We conducted two analyses; one including data from the four centers and one excluding data from Center 2, as the methods in this center differed from the others.

Results: In the first analysis, we found no differences in $\mathrm{LICl}$ between the different subject types. In all subjects, the response ratios at ISIs 100 and 150 ms showed significantly more inhibition than the response ratios at ISIs 50, 200 and $250 \mathrm{~ms}$. Our second analysis showed a significant interaction between ISI and subject type ( $p=0.0003$ ). Post hoc testing showed significant differences between controls and refractory focal epilepsy at ISIs of $100 \mathrm{~ms}(p=0.02)$ and $200 \mathrm{~ms}(p=0.04)$. There were no significant differences between the control and refractory generalized epilepsy groups or between the refractory generalized and focal epilepsy groups.

Conclusion: Our results do not support the body of previous work that suggests that $\mathrm{LICl}$ is significantly reduced in refractory focal and genetic generalized epilepsy. Results from the second analysis are even in sharper contrast with previous work, showing inhibition in refractory focal epilepsy at ISI 200 ms instead of facilitation previously reported. Methodological differences, especially shorter intervals between the pulse pairs, may have contributed to our inability to reproduce previous findings. Based on our results, we suggest that $\mathrm{LICl}$ as measured by TMS-EMG is unlikely to have clinical use as a biomarker for epilepsy. 


\subsection{Introduction}

Epilepsy is a paroxysmal neurological condition characterized by an enduring predisposition to generate epileptic seizures (Fisher et al., 2014). The diagnosis is based on the clinical history, often supported by interictal or ictal epileptic discharges on the electroencephalogram (EEG) (Rosenow et al., 2015). These pathological changes in the EEG are paroxysmal and do not always occur during a short EEG recording (Smith, 2005). The diagnostic sensitivity of routine EEG for epilepsy is estimated at $17 \%$ in adults and at $58 \%$ in children (Bouma et al., 2016). Sensitivity can be moderately increased by increasing recording time, or by using activation procedures such as sleep deprivation, hyperventilation or photic stimulation (Rosenow et al., 2015; Smith, 2005). In about $70 \%$ of those diagnosed with epilepsy, seizures can be suppressed with antiepileptic drugs (AEDs) (Brodie et al., 2012), but finding the optimal AED type and dose for an individual patient can be a difficult and time-consuming process. The EEG does not provide a direct measure of seizure proneness and its use in the follow-up of epilepsy is therefore limited, spurring the search for a reliable biomarker of epilepsy activity to improve its management (Engel, 2008; Smith, 2005).

Increased cortical excitability resulting from an imbalance between excitatory and inhibitory activity is thought to play an important role in the pathophysiology of epilepsy (Schwartzkroin, 1994). Cortical excitability can be measured non-invasively using single or paired pulse transcranial magnetic stimulation (TMS) (Reutens and Berkovic, 1992). Single pulse protocols are used to assess the motor threshold (MT), motor evoked potential (MEP) amplitude and cortical silent period. With paired pulse protocols, short-interval intracortical inhibition, intracortical facilitation and longinterval intracortical inhibition (LICl) can be measured (Kobayashi and Pascual-Leone, 2003). Several studies showed increased cortical excitability in groups of drug naïve subjects with generalized or focal epilepsy compared to healthy controls, for a review see de Goede et al. (2016). Four studies reported a decrease in excitability after successful treatment with AEDs but not after ineffective treatment (Badawy et al., 2010a, 2013a,d,e).

Of the different variables measured with TMS, those measured with paired pulse protocols appear to hold the greatest potential as biomarkers for epilepsy for several reasons: first, they provide information about cortical excitability rather than integrated cortico-spinal excitability as is the case for single pulse measures (Ziemann et al., 1996a). Second, they can be expressed as dimensionless ratios, enabling comparison across different institutions. Third, paired pulse protocols appear to yield more reliable findings than single pulse protocols (de Goede et al., 2016). Several studies consistently found facilitation at the short ( 2 and $5 \mathrm{~ms}$ ) and long (250 and 300 $\mathrm{ms}$ ) interstimulus intervals (ISIs), instead of inhibition as in healthy controls, providing 
evidence for cortical hyperexcitability in drug naïve epilepsy, for a review see de Goede et al. (2016). Lastly, this difference between facilitation in drug naïve epilepsy and inhibition in controls was larger at long than at short ISIs (Badawy et al., 2007). Of the different TMS variables, LICI may thus be the most suitable as an epilepsy biomarker. $\mathrm{LICl}$ is measured by applying two supra-threshold stimuli with an ISI of 50-400 ms and calculating the ratio between the response to the second (test stimulus) and to the first (conditioning stimulus). A variant consists of the ratio between the response to the test stimulus and to an unconditioned stimulus. A ratio with values $<1$ indicates inhibition, while values $>1$ indicate facilitation (Valls-Solé et al., 1992). LICl is thought to be linked to GABA-B receptor mediated inhibition (McDonnell et al., 2006; Werhahn et al., 1999).

In epilepsy, $\mathrm{LICl}$ was mainly studied by one group of investigators who, in several studies, showed facilitation instead of inhibition at ISIs 50, 150, 250 and $300 \mathrm{~ms}$ in groups of drug naïve subjects with different types of genetic generalized epilepsy (Badawy et al., 2007, 2010a, 2012, 2013a,c,d, 2014b; Badawy and Jackson, 2012; Brodtmann et al., 1999). In drug naïve focal epilepsy, cortical excitability was consistently increased in the hemisphere ipsilateral to the epileptic focus, but not in the contralateral hemisphere, at ISIs 250 and 300 ms (Badawy et al., 2007, 2010a, 2012, 2013a,b,c,e, 2014b, 2015; Badawy and Jackson, 2012). In successfully treated epilepsy, hyperexcitability normalized over time in seizure-free groups, becoming more similar to controls, but it remained increased in refractory groups (Badawy et al., 2010a, 2013a,d,e). Two recent studies from other groups reported contrasting findings, however, the first found significantly lower cortical excitability in subjects with genetic generalized epilepsy on AEDs compared to a healthy control group and a drug naïve generalized epilepsy group at ISIs between 200-250 ms. No significant differences were found between the drug naïve epilepsy and control groups (Silbert et al., 2015). The second study found inhibition at an ISI of $50 \mathrm{~ms}$ in poorly controlled epilepsy, but not in moderately controlled epilepsy or healthy controls. At an ISI of $200 \mathrm{~ms}$, the groups with poorly and moderately controlled epilepsy both showed inhibition (more prominent in the poorly controlled group), whereas healthy controls did not. These results, however, were not significant after correction for multiple comparisons (Pawley et al., 2017).

To establish the true potential of $\mathrm{LICl}$ as a clinical biomarker for epilepsy, the promising findings need to be replicated and extended to larger groups. An ideal biomarker needs to provide consistent results across different centers. For it to be useful on an individual level, the inter-individual variability should be low. A difference in excitability between refractory epilepsy and healthy controls would support the use of $\mathrm{LICl}$ to rapidly evaluate the effect of AED treatment. We report on $\mathrm{LICl}$ data from healthy controls and subjects with refractory generalized or focal epilepsy from four centers in two different countries. Our results do not support the utility of $\mathrm{LICl}$ as a biomarker for epilepsy. 


\subsection{Materials and methods}

Data were collected independently in four different tertiary referral centers, two each in the Netherlands and the United Kingdom (UK), and retrospectively pooled. The centers were: 1) Medisch Spectrum Twente (the Netherlands), 2) Stichting Epilepsie Instellingen Nederland - SEIN (the Netherlands), 3) King's College London (UK) and 4) University College London (UK).

The studies were performed in accordance with guidelines for TMS use in clinical practice and research (Rossi et al., 2009). All study protocols were approved by the local ethics committees of each of the participating centers.

\subsubsection{Subjects}

Informed written consent was provided by all participants. For those younger than 18 years, assent was also obtained from both parents. People with contra-indications to TMS other than epilepsy and pregnant females were excluded.

\section{Center 1}

Healthy adults (18 years or older) were recruited locally through advertisement at the University of Twente and the Medisch Spectrum Twente. People with a history of epilepsy, brain lesions or spinal cord surgery were excluded. Hand dominance was assessed with the Dutch Handedness Questionnaire (van Strien, 1992, 2003).

\section{Center 2}

Healthy participants (12 years or older) were recruited locally through digital and paper adverts. People with a neurological or psychiatric condition, including migraine or epilepsy, diabetes mellitus and people taking medication that could affect cortical excitability (such as psychoactive drugs and $\beta$-blockers) were excluded. Hand dominance was assessed with the Dutch version of the Edinburgh handedness questionnaire (Oldfield, 1971).

\section{Center 3}

Adults with a clinical diagnosis of epilepsy were recruited via specialized neurology and epilepsy clinics at King's College Hospital, St Thomas' Hospital, St George's Hospital, London, Kent and Canterbury Hospital and Queen Elizabeth Hospital, Woolwich. For the control group, healthy adults without a personal or family history of neurological or psychiatric conditions were recruited through a local research volunteer's database and friends of participants. Those with epilepsy who had a neuropsychiatric condition other than epilepsy, non-epileptogenic seizures, an estimated IQ $<70$ or who did not cooperate with the TMS procedures were excluded. In part of the cohort, hand dominance was assessed with the Edinburgh handedness questionnaire (Oldfield, 1971). 


\section{Center 4}

Adults with epilepsy were recruited through specialized epilepsy clinics and an inpatient unit at the National Hospital for Neurology and Neurosurgery (Queen Square and Chalfont sites). Participants with a clinical diagnosis of refractory genetic generalized or focal epilepsy were included. Hand dominance was assessed with the Edinburgh handedness questionnaire (Oldfield, 1971).

\subsubsection{Data acquisition}

In all centers, participants were seated in a comfortable chair with their hands in a relaxed position and their eyes open. The experimental set-up and stimulation protocol of each center is summarized in Table 7.1.

\section{Center 1}

TMS was performed with a Magstim Rapid ${ }^{2}$ Stimulator (maximum stimulator output $0.8 \mathrm{~T}$ ) and a figure-of-eight air film $70 \mathrm{~mm}$ coil (The Magstim Company Ltd, Whitland, UK). Biphasic TMS pulses were given to both motor hotspots of the abductor digiti minimi (ADM) muscle. Muscle activity was recorded using two surface $\mathrm{Ag} / \mathrm{AgCl}$ electrodes placed in a belly-tendon montage. MEPs were recorded from the contralateral ADM muscle with a 72-channel Refa system (TMSi, Oldenzaal, the Netherlands). Data were recorded with a sampling frequency of $5 \mathrm{kHz}$ and stored for offline analysis. Measurements were conducted between 9 AM-5 PM.

\section{Center 2}

TMS was performed with a MagPro X100 magnetic stimulator (maximum stimulator output $3.9 \mathrm{~T}$ ) and a $12 \mathrm{~cm}$ diameter parabolic circular MMC-140 coil (Magventure, Farum, Denmark). Biphasic TMS pulses were given on the vertex $(\mathrm{Cz})$. MEPs were recorded bilaterally from the abductor pollicis brevis (APB) muscles with a Nicolet Viking EDX electromyography (EMG) system (Natus, Madison, WI, USA). Data were recorded with a sampling frequency of $4 \mathrm{kHz}$ and stored for offline analysis. Measurements were conducted between 9 AM-4 PM.

\section{Center 3}

TMS was performed using two Magstim $200^{2}$ stimulators connected via a BiStim module and a figure-of-eight $90 \mathrm{~mm}$ coil (The Magstim Company Ltd, Whitland, UK). Monophasic TMS pulses were given to both motor hotspots of the first dorsal interosseous (FDI) muscle. MEPs were recorded from the contralateral FDI muscle with a CED1902 EMG amplifier and CED 1401 Signal 3.13 software (Cambridge Electronic Design, Cambridge, UK) using a sampling rate of $15 \mathrm{kHz}$, a bandwidth of $10-5000 \mathrm{~Hz}$, 
Table 7.1: TMS set-up and stimulation protocol per center.

\begin{tabular}{|c|c|c|c|c|}
\hline & Center 1 & Center 2 & Center 3 & Center 4 \\
\hline Stimulator type & Magstim Rapid ${ }^{2}$ & MagPro X100 & Magstim BiStim² & Magstim BiStim ${ }^{2}$ \\
\hline $\begin{array}{l}\text { Maximum stimulator } \\
\text { output }(\mathrm{T})\end{array}$ & 0.8 & 3.9 & 1.5 & 1.5 \\
\hline $\begin{array}{l}\text { Magnetic pulse } \\
\text { waveform }\end{array}$ & biphasic & biphasic & monophasic & monophasic \\
\hline Coil type & figure-of-eight & circular & figure-of-eight & figure-of-eight \\
\hline Stimulation location & motor hotspot & vertex $(\mathrm{Cz})$ & motor hotspot & motor hotspot \\
\hline Target muscle & ADM & APB & FDI & APB \\
\hline Intertrial interval & $\sim 4 \mathrm{~s}$ & $1 \mathrm{~s}$ & $4 \mathrm{~s}$ & $5 \mathrm{~s}$ \\
\hline Repetitions per ISI & 50 & 6 & 10 & 10 \\
\hline Order of ISIs & random & increasing & random & random \\
\hline $\begin{array}{l}\text { Conditioning pulse } \\
\text { intensity ( } \% \text { rMT) }\end{array}$ & 120 & 110 & 120 & 110 \\
\hline $\begin{array}{l}\text { Test pulse intensity } \\
\text { (\% rMT) }\end{array}$ & 120 & 110 & 120 & 110 \\
\hline $\mathrm{LICl}$ response ratio & $\begin{array}{l}\text { mean }(T R) / \\
\text { mean }(C R)\end{array}$ & $\begin{array}{c}\text { mean(TR)/ } \\
\text { mean(uncMEP) }\end{array}$ & mean (TR/CR) & $\begin{array}{c}\text { mean(TR)/ } \\
\text { mean(uncMEP) }\end{array}$ \\
\hline $\begin{array}{l}\text { Time of day } \\
\text { measurement }\end{array}$ & $9 \mathrm{AM}-5 \mathrm{PM}$ & 9AM-4 PM & $9 \mathrm{AM}-5 \mathrm{PM}$ & $9 \mathrm{AM}-5 \mathrm{PM}$ \\
\hline
\end{tabular}

a gain of 1000 (ranging from -5 to $5 \mathrm{~V}$ ) and traces recorded on Signal 3.13 software and stored for offline analysis. Measurements were conducted between 9 AM-5 PM.

\section{Center 4}

Hardware and software for TMS-EMG data collection were the same as in Center 3. A figure-of-eight $70 \mathrm{~mm}$ D70 alpha coil was used (The Magstim Company Ltd, Whitland, UK). Monophasic TMS pulses were given to the dominant motor hotspot of the APB muscle. MEPs were recorded with a sampling frequency of $2 \mathrm{kHz}$ and stored for offline analysis. Recordings were obtained between 9 AM-5 PM.

\subsubsection{Estimation of the resting motor threshold}

For the three centers using a figure-of-eight coil, the resting motor threshold (rMT) was determined by applying single pulses to the ADM (Center 1), FDI (Center 3) or APB (Center 4) motor hotspots. The hotspot was defined as the location were the largest 
MEPs were induced when the TMS coil was placed tangentially with the handle pointed backwards and laterally at an angle of $45^{\circ}$ from the midline. Stimulation commenced at $30 \%$ of maximum stimulator output and increased in $5 \%$ increments until a MEP was seen. One per cent changes in intensity were then used to find the threshold, defined as the minimum stimulus intensity which produced a MEP with a peak-to-peak amplitude $>50 \mu \mathrm{V}$ (>100 $\mu \mathrm{V}$ in Center 4$)$ in $50 \%$ or more of ten trials in the fully relaxed target muscle (Rossini et al., 1994). Relaxation of the target muscle was monitored by continuous visual observation of the EMG.

The approach was different in Center 2, where the rMT was approximated using a single pulse stimulus-response curve, with the coil on the vertex. Stimulation started at $20 \%$ of stimulator output with $5 \%$ stepwise increments until there was a consistent twitch in the hand contralateral to the stimulated hemisphere in $50 \%$ or more of eight trials (approximated rMT). Then, a semi-automated, in-house designed scanning protocol (created in Matlab (version 7.5.0 R2007b, The MathWorks, Natick, MA, USA)) was used to automatically deliver stimuli with a fixed intertrial interval of $2 \mathrm{~s}$, and eight stimuli at each intensity. Scanning started at a stimulator output value of $10-12 \%$ below the approximated $\mathrm{rMT}$ and increased in $2 \%$ steps until a reproducible MEP ( $>200 \mu \mathrm{V}$ ) was seen after every stimulus (corresponding to $110-120 \%$ rMT). The rMT was defined as the lowest stimulus intensity eliciting a visible twitch in any hand muscle in $50 \%$ or more of eight stimuli (Varnava et al., 2011).

\subsubsection{Assessment of long-interval intracortical inhibition}

\section{Center 1}

Paired pulse stimulation was applied with the coil over the ADM motor hotspot. Both pulses were given at $120 \%$ rMT with ISIs between $50-300 \mathrm{~ms}$, with 50 ms increments (six intervals). Stimulation was repeated 50 times for each ISI. The stimulus pairs were given randomly with $\sim 4 \mathrm{~s}$ (range 3.5-4.5 s) between stimulus pairs (intertrial interval). To calculate the $\mathrm{LICI}$ for each ISI, the ratio was taken between the mean peak-topeak amplitude of the responses to the second (test) stimuli (test responses (TR)) and the mean peak-to-peak amplitude of the responses to the first (conditioning) stimuli (conditioning responses (CR)): mean(TR)/mean(CR).

\section{Center 2}

Paired pulse stimulation was applied with the coil over the vertex. Both pulses were given at $110 \%$ rMT, with increasing ISIs between 50-400 ms, with $25 \mathrm{~ms}$ increments (15 intervals). Stimulation was repeated six times for each ISI. The stimulus pairs were given in a fixed increasing order with an intertrial interval of $1 \mathrm{~s}$. An unconditioned stimulus was given six times immediately before the start of 
the paired pulse stimulation protocol. To calculate the $\mathrm{LICl}$ for each ISI, the mean peak-to-peak amplitude in response to the conditioned second stimuli (TR) was divided by the mean peak-to-peak amplitude in response to the unconditioned stimuli: mean(TR)/mean(unconditioned MEP).

\section{Center 3}

Paired pulse stimulation was applied with the coil over the FDI motor hotspot. Both paired pulses were given at 120\% rMT. Four ISIs were tested: 50, 150, 200 and 250 ms. Stimulation was repeated ten times for each ISI. The stimulus pairs were given in random order with an intertrial interval of $4 \mathrm{~s}$. To calculate the $\mathrm{LICI}$ for each ISI, the ratios were taken between the peak-to-peak amplitudes of the responses to the second test stimuli (TR) and the peak-to-peak amplitudes of the responses to the first conditioning stimuli (CR). Then the mean over all ratios was taken: mean(TR/CR).

\section{Center 4}

Paired pulse stimulation was applied with the coil over the APB motor hotspot. Both pulses were given at 110\% rMT. Five ISIs were tested: 50, 100, 150, 200 and 250 ms. Stimulation was repeated ten times for each ISI. Stimulus pairs were given in a random order with a 5 s intertrial interval. LICI was calculated in the same way as in Center 2.

\subsubsection{Data analysis}

Each center provided the following individual data for analysis: age, gender, hand dominance (if available), epilepsy diagnosis, including whether epilepsy was refractory to treatment with AEDs (defined as at least one seizure in the year preceding the TMS measurement), number of different AEDs, side of seizure focus (in case of focal epilepsy) and the mean $\mathrm{LICl}$ for each ISI. Only $\mathrm{LICI}$ values of healthy controls and subjects with refractory epilepsy (generalized or focal) were included in the analysis. Based on previous reports, cortical excitability as measured by $\mathrm{LICI}$ remains elevated in those who are refractory to pharmacological treatment, whereas it normalizes (returns to the levels seen in controls) in those who become seizure-free. The refractory epilepsy group is, therefore, the most interesting to assess in this context. For controls and genetic generalized epilepsy, we only analyzed the LICI when stimulating the dominant hemisphere (left hemisphere for right-hand dominance). When hand dominance was unknown and in ambidextrous participants, we analyzed the $\mathrm{LICl}$ when stimulating the left hemisphere. For focal epilepsy, we included the $\mathrm{LICl}$ when stimulating the hemisphere ipsilateral to the epileptic focus in the analysis, or the dominant/left hemisphere when epileptic foci were bilateral. We included the $\mathrm{LICl}$ measured at ISIs $50,100,150,200$ and $250 \mathrm{~ms}$. 


\subsubsection{Statistical analysis}

Data analysis was done in R (RCore-Team, 2015). As the material and stimulation protocols differed between the centers, we expected not only large inter-individual variability of $\mathrm{LICl}$ values, but also a large variability between the centers. This intercenter variability limits the comparison of data between the centers and means that data cannot simply be pooled for analysis. Linear mixed-effects models (Ime4 package, Linear Mixed Effects version 4 (Bates et al., 2015)) are the best way to deal with such datasets, as they allow for correction of systematic variability. We accounted for the heterogeneity of $\mathrm{LICl}$ values across subjects and centers by defining them as effects with a random intercept, thus instructing the model to correct for any systematic differences between the subjects (inter-individual variability) and centers (intercenter variability). We then analyzed the influence of two possible fixed effects on LICI: 1) the subject type (three levels: controls, refractory genetic generalized epilepsy and refractory focal epilepsy) and 2) the ISIs (five levels: 50, 100, 150, 200 and $250 \mathrm{~ms}$ ). LICI response ratios were log transformed to better approximate normality, see Figure A of the Supplementary material. To optimize our model, we checked the normality of the model residual.

We ran a type II analysis of variance. Wald chi-square tests were used for fixed effects in linear mixed-effects models. For post hoc tests we used the Lsmean package (Lsmean version 2.20-23, (Searle et al., 1980)) where $p$-values were considered as significant at $p<0.05$ and adjusted for the number of comparisons performed (Tukey method).

\subsection{Results}

\subsubsection{Subjects}

\section{Center 1}

Twenty-five healthy subjects were included. Four were excluded from the analyses: in two, stimulation was not possible at an intensity of $120 \% \mathrm{rMT}$, in one the session was terminated prematurely as the subject felt unwell and in one, $\mathrm{LICl}$ data were not available from the dominant hemisphere. Twenty-one healthy individuals were included in the analysis ( 6 males, mean age 28.6 years; range 20-49 years, 3 lefthanded), see Figure 7.1.

\section{Center 2}

Thirty-eight controls were included; one was excluded due to non-specific EEG abnormalities. Data of 37 controls were included in the analysis (11 males, mean age 38.1 years; range 15-62 years, 4 left-handed; 1 ambidextrous), see Figure 7.1. 
Center 1

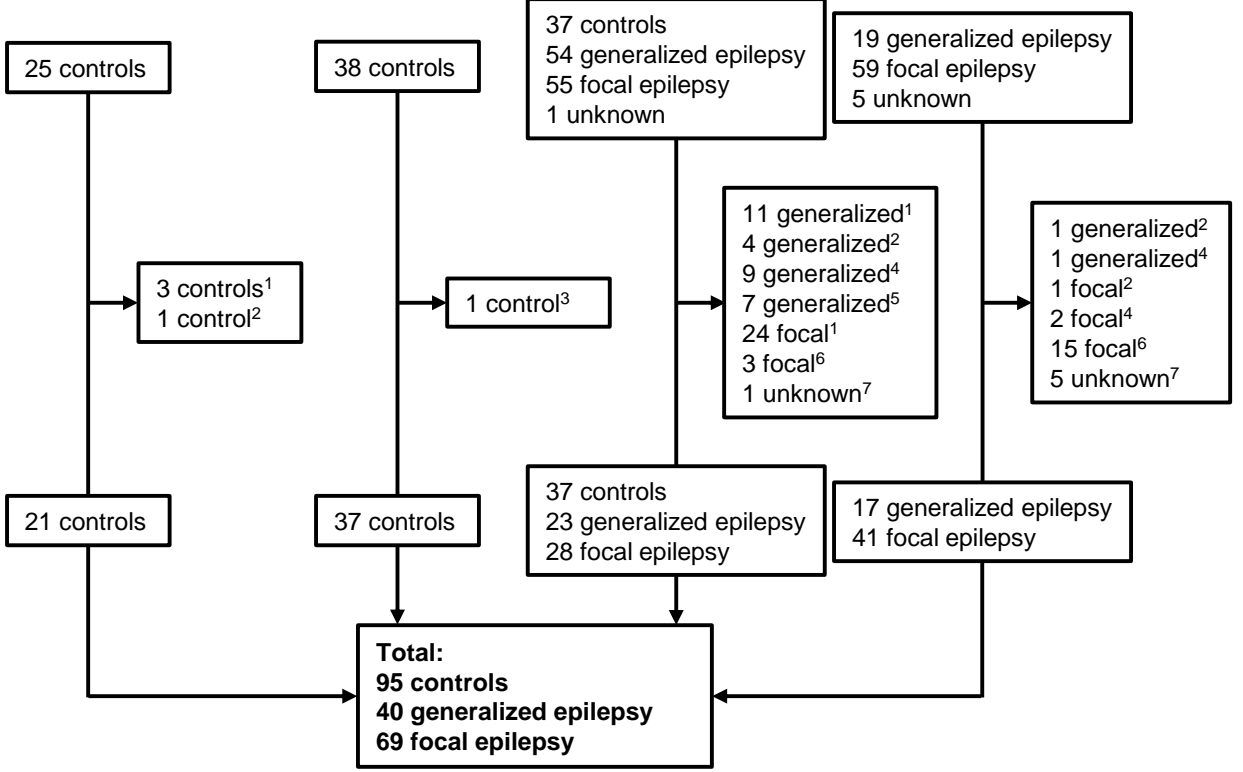

Figure 7.1: Included and excluded subjects per center. Exclusion criteria: ${ }^{1} \mathrm{No} \mathrm{LICl}$ data; ${ }^{2} \mathrm{No}$ $\mathrm{LICl}$ data for the dominant hemisphere; ${ }^{3} \mathrm{Aspecific}$ abnormalities on the EEG; ${ }^{4} \mathrm{No}$ treatment with AEDs; ${ }^{5}$ Not refractory; ${ }^{6} \mathrm{No} \mathrm{LICl}$ data for the hemisphere ipsilateral to the epileptic focus; and ${ }^{7}$ Type of epilepsy unknown.

\section{Center 3}

Thirty-seven controls and 110 subjects with epilepsy were included (54 with genetic generalized epilepsy, 55 with focal epilepsy and one with an unclear diagnosis). All controls were included in the analysis (19 males, mean age 30.2 years; range 18-52 years, 4 left-handed; 1 ambidextrous). Of the 54 subjects with generalized epilepsy, 31 were excluded from the analysis: in eleven $\mathrm{LICl}$ data were not collected (for reasons including too high rMT or discomfort during stimulation), in four $\mathrm{LICl}$ data was not available from the dominant hemisphere, nine were not taking AEDs at the time of the experiment and seven were not considered refractory. Thus, 23 subjects with refractory genetic generalized epilepsy were included in the analysis (10 males, mean age 30.1 years; range 18-54 years, hand dominance known in 12; of these 2 were left-handed, AEDs: median 1; range 1-4). In 24 of the 55 with focal epilepsy, $\mathrm{LICl}$ data were not collected. In three, $\mathrm{LICl}$ data were only available for the hemisphere contralateral to the epileptic focus. Thus, 28 subjects with refractory focal epilepsy were included in the analysis (12 males, mean age 39.4 years; range 21-66 years, AEDs: median 1; range 1-3), see Figure 7.1. The hand dominance of this group was unknown. 


\section{Center 4}

Nineteen participants with genetic generalized epilepsy were included. One had to be excluded from the analysis as no AEDs was used and one as $\mathrm{LICl}$ data from the dominant hemisphere was not available. Seventeen subjects with genetic generalized epilepsy were included in the analysis (8 males, mean age 34.4 years; range 20-51 years, 1 lefthanded; 2 ambidextrous, AEDs: median 3; range 1-5). Fifty-nine with focal epilepsy were included but fifteen were excluded as $\mathrm{LICl}$ data from the hemisphere ipsilateral to the epileptic focus were not available. In one participant with a bilateral focus, LICI data from the dominant hemisphere were unavailable. Two participants were not taking AEDs, leaving 41 with refractory focal epilepsy for analysis (18 males, mean age 39.7 years; range 18-61 years, 4 left-handed, AEDs: median 2; range 1-6), see Figure 7.1. A further five participants were included, but as the epilepsy diagnosis was unclear they were excluded from the current analysis.

In total, we included data from 204 subjects in the analysis, 40 with refractory generalized epilepsy, 69 with refractory focal epilepsy and 95 healthy controls, see Figure 7.1. Part of the data from this cohort was previously reported in other studies (Center 1: de Goede and van Putten (2017), Center 3: Chowdhury et al. (2015) and Pawley et al. (2017) and Center 4 (including patients with Dravet Syndrome): Stern et al. (2016, 2017)).

\subsection{2 $\mathrm{LICl}$ recovery curves}

For all subject types, the response ratios for each ISI are shown in Figure 7.2 and Table 7.2. As expected, we found inhibition (median $\mathrm{LICl}$ value $<1$ ) in healthy controls at all analyzed ISIs (50, 100, 150, 200 and $250 \mathrm{~ms}$ ), see Figure 7.2 and Figure B of the Supplementary material. Unexpectedly, the median $\mathrm{LICl}$ was also $<1$ in refractory genetic generalized epilepsy (all ISIs except $50 \mathrm{~ms}$ ) and refractory focal epilepsy (all ISIs). The mean $\mathrm{LICl}$ also showed inhibition in the controls, except in Center 2 (all ISIs), in Center 1 at ISI 50 ms and in Center 3 at ISI 250 ms, see Table 7.2. In the refractory epilepsy groups the mean $\mathrm{LICl}$ was also $<1$ at most ISIs, except for genetic generalized epilepsy at ISI 50 ms and focal epilepsy in Center 3 at ISI 250 ms, see Table 7.2. The linear mixed-effects model showed that there was no significant interaction between subject type and $\mathrm{LICl}$ at any of the ISIs. We found a main effect of interaction between ISI and $\mathrm{LICI}(p<0.001)$. Post hoc tests revealed that $\mathrm{LICI}$ at ISIs 100 and $150 \mathrm{~ms}$ showed significantly more inhibition compared to $\mathrm{LICl}$ at ISIs 50, 200 and $250 \mathrm{~ms}$ for all three subject types, see Figure 7.3. The methods of Center 2 differ the most from those used in the other centers, as it was the only site to use a circular coil and stimulation on the vertex. It also used the shortest intertrial interval and the lowest number of repetitions per ISI. The statistical model takes this methodological heterogeneity into 


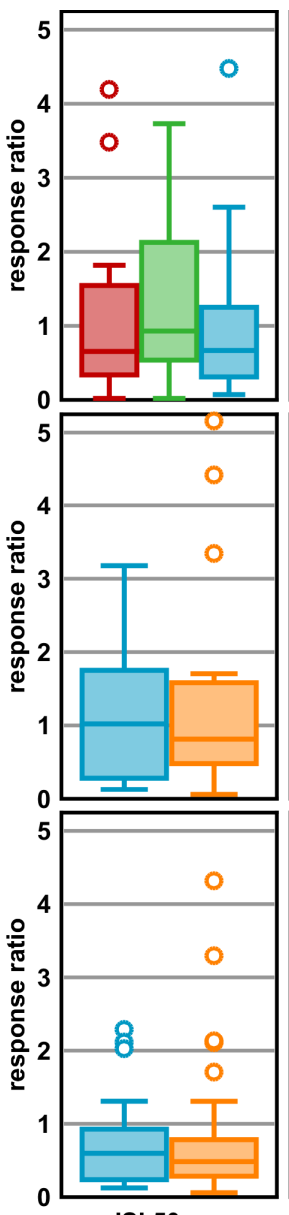

ISI 50 ms
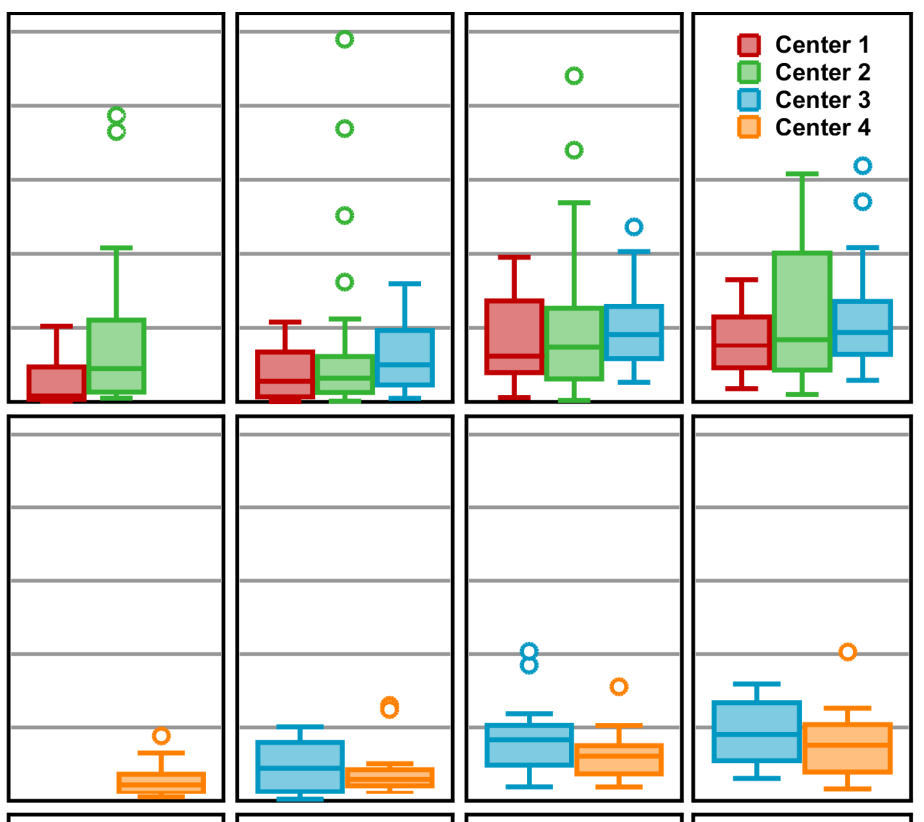

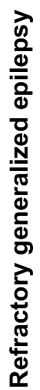

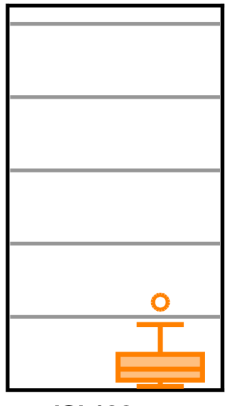

ISI $100 \mathrm{~ms}$

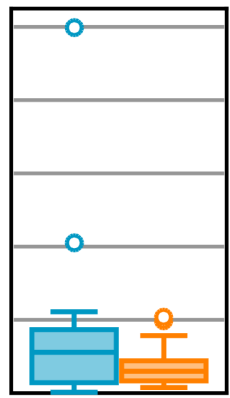

ISI $150 \mathrm{~ms}$

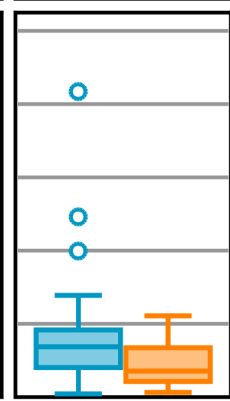

ISI $200 \mathrm{~ms}$

Figure 7.2: $\mathrm{LICl}$ at each ISI per center and subject type. The boxplots show the median $\pm 25^{\text {th }}$ percentiles; the whiskers show 1.5 times the interquartile range (IQR); the dots show outliers outside the whiskers. All outliers were included in the analysis but several are outside the boundaries of the $y$-axis of the figure: healthy controls - Center 1: $\mathrm{LICl}=7.00$ (ISI $50 \mathrm{~ms}$ ); Center 2: $\mathrm{LICl}=5.37,5.42,18.50,24.20,45.24$ (ISI $50 \mathrm{~ms}), 5.56,6.02,7.37$ (ISI $100 \mathrm{~ms}$ ), 10.23, 10.31 (ISI $150 \mathrm{~ms}), 6.15,9.68$ (ISI $200 \mathrm{~ms}), 6.11,6.61$ (ISI $250 \mathrm{~ms}$ ). Generalized epilepsy - Center 3: $\mathrm{LICI}=$ 8.86 (ISI $50 \mathrm{~ms}$ ). Focal epilepsy - Center 3: LICI = 6.73 (ISI $250 \mathrm{~ms}$ ). 


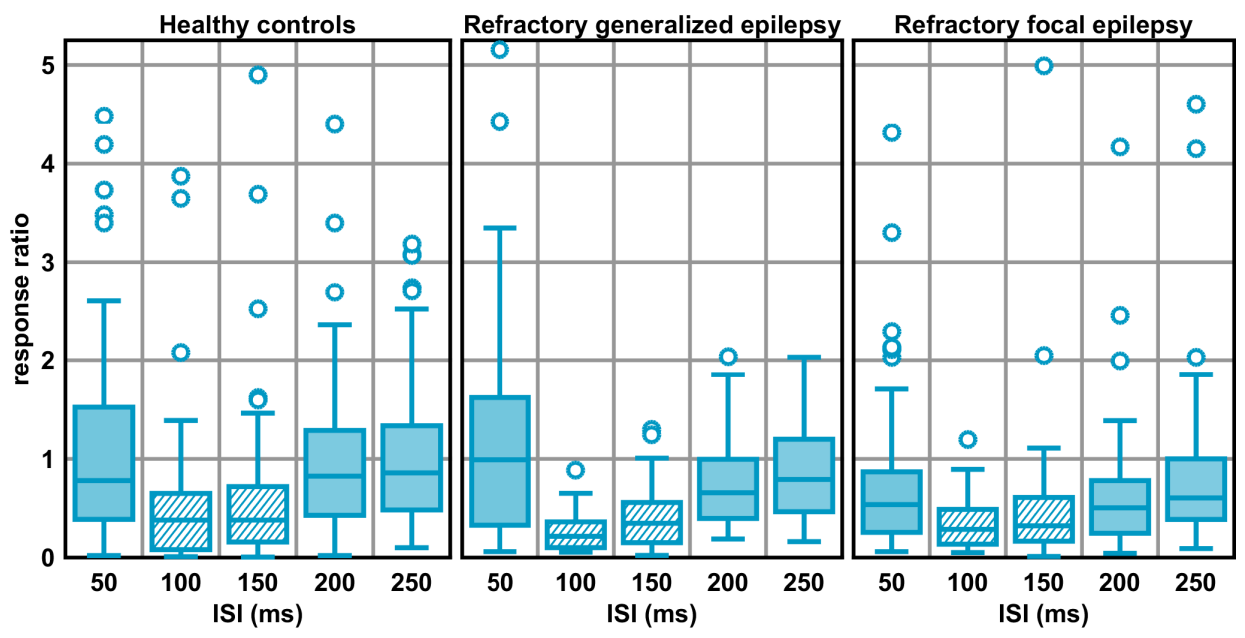

Figure 7.3: $\mathrm{LICl}$ at all ISIs per subject type pooled across Centers 1-4. The boxplots show the median $\pm 25^{\text {th }}$ percentiles; the whiskers show 1.5 times the IQR. LICI at ISIs 100 and $150 \mathrm{~ms}$ (diagonal pattern) shows significantly more inhibition than LICI at ISIs 50, 200 and $250 \mathrm{~ms}$ for all subject types.

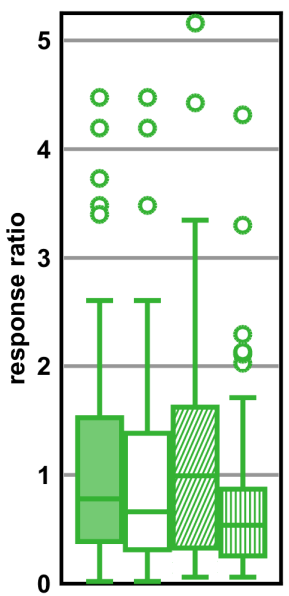

ISI $50 \mathrm{~ms}$

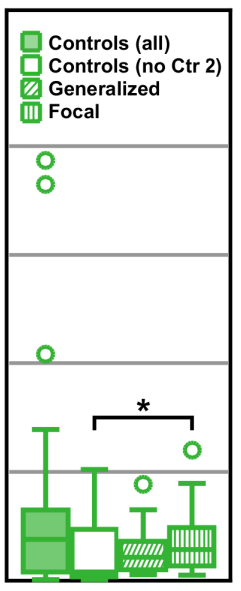

ISI $100 \mathrm{~ms}$

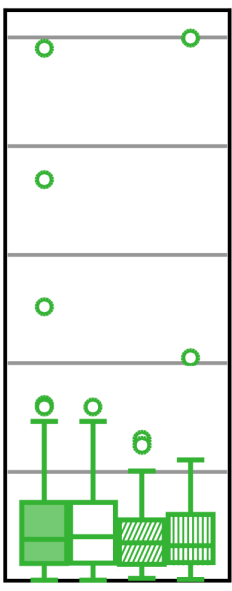

ISI $150 \mathrm{~ms}$

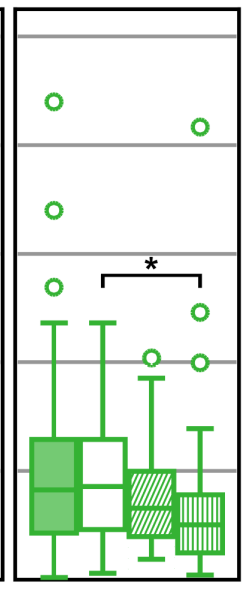

ISI $200 \mathrm{~ms}$

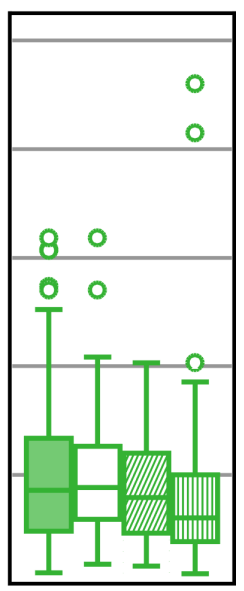

ISI $250 \mathrm{~ms}$

Figure 7.4: $\mathrm{LICl}$ at all ISIs per subject type pooled across Centers 1-4. The boxplots show the median $\pm 25^{\text {th }}$ percentiles; the whiskers show 1.5 times the IQR. ${ }^{*}$ Significant difference between controls (open boxes, without Center 2 ) and refractory focal epilepsy at ISIs $100 \mathrm{~ms}(p=0.02)$ and $200 \mathrm{~ms}(p=0.04)$. There were no significant differences between the control (open boxes, without Center 2) and refractory generalized epilepsy group or between the refractory generalized and focal epilepsy groups. There were no differences between the groups when data from all centers were included in the analysis (filled boxes). 
Table 7.2: $\mathrm{LICl}($ mean \pm SD) at each ISI per center and subject type.

\begin{tabular}{lcccccc}
\hline \multicolumn{2}{c}{ Subject type Center } & ISI $50 \mathrm{~ms}$ & ISI $100 \mathrm{~ms}$ & ISI $150 \mathrm{~ms}$ & ISI $200 \mathrm{~ms}$ & ISI $250 \mathrm{~ms}$ \\
\hline \multirow{2}{*}{ Controls } & $\mathbf{1}$ & $1.32 \pm 1.69$ & $0.28 \pm 0.30$ & $0.39 \pm 0.36$ & $0.81 \pm 0.55$ & $0.82 \pm 0.40$ \\
& 2 & $3.76 \pm 8.74$ & $1.14 \pm 1.80$ & $1.15 \pm 2.43$ & $1.32 \pm 1.91$ & $1.37 \pm 1.50$ \\
Generalized & $\mathbf{3}$ & $1.39 \pm 1.84$ & - & $0.45 \pm 0.34$ & $0.82 \pm 0.47$ & $0.91 \pm 0.42$ \\
epilepsy & $\mathbf{4}$ & $1.37 \pm 1.51$ & $0.27 \pm 0.23$ & $0.40 \pm 0.35$ & $0.63 \pm 0.34$ & $0.78 \pm 0.49$ \\
Focal & $\mathbf{3}$ & $0.73 \pm 0.60$ & - & $0.70 \pm 0.95$ & $0.88 \pm 0.85$ & $1.27 \pm 1.36$ \\
epilepsy & $\mathbf{4}$ & $0.78 \pm 0.89$ & $0.33 \pm 0.25$ & $0.34 \pm 0.24$ & $0.46 \pm 0.28$ & $0.64 \pm 0.66$ \\
\hline
\end{tabular}

For controls and refractory genetic generalized epilepsy $\mathrm{LICl}$ measured when stimulating the dominant hemisphere is shown, for refractory focal epilepsy when stimulating the ipsilateral hemisphere.

account but as Center 2 also seems to have less consistent results and more outliers than Centers 1 and 3 and other studies in the field (Cash et al., 2010; Caux-Dedeystère et al., 2015; Silbert et al., 2015; Valls-Solé et al., 1992; Wassermann et al., 1996), we reran the statistical analysis without Center 2 data. In contrast with the first analysis, this showed a significant interaction between ISI and subject groups $(p=0.0003)$. Post hoc testing revealed a small, yet significant difference between controls and refractory focal epilepsy at ISIs $100 \mathrm{~ms}(p=0.02)$ and $200 \mathrm{~ms}(p=0.04)$. There were no significant differences between the control and refractory generalized epilepsy groups or between the refractory generalized and focal epilepsy groups, see Figure 7.4. At an ISI of $100 \mathrm{ms,}$ the $\mathrm{LICl}$ of both controls and refractory focal epilepsy was $<1$, indicating inhibition. At an ISI 200 of ms, there was neither inhibition nor facilitation in the controls $(\mathrm{LICl} \sim 1)$ but inhibition in the focal epilepsy group $(\mathrm{LICl}<1)$.

\subsection{Discussion}

Our analysis of long-interval paired pulse TMS data collected in four different centers do not support previous promising findings (Badawy et al., 2010a, 2013a,d,e). Our first analysis, including data from all centers, showed no significant differences in $\mathrm{LICl}$ between subjects with refractory genetic generalized epilepsy, subjects with refractory focal epilepsy and healthy controls. We observed a statistically significant difference between the ISIs 50, 200 and 250 ms on one hand, and ISIs 100 and 150 ms on the other hand for all subjects. Inhibition was measured at all five ISIs in all subject types but it was significantly stronger at ISIs 100 and $150 \mathrm{~ms}$. The results from our second analysis without Center 2 data, clearly contrast with previous findings (Badawy et al., 2010a, 2013a,d,e). First, we find inhibition in the refractory epilepsy groups instead of facilitation $(\mathrm{LICl}>1)$ reported previously. Second, the differences in our 
sample are only found between refractory focal epilepsy and controls and not in generalized refractory epilepsy. Third, the differences are found at other ISIs (100 and $200 \mathrm{~ms}$ instead of 50, 150 and $250 \mathrm{~ms}$ ) than previously reported (Badawy et al., 2010a, 2013a,d,e). Lastly, the differences between controls and refractory focal epilepsy are much smaller in our sample than in previous reports (Badawy et al., 2010a, 2013a,d,e).

One of the main methodological differences between our study protocols and that of previous studies is the intertrial interval, which ranged between 1-5 s in our studies but was 15 s in previous studies (Badawy et al., 2007, 2010a,b, 2012, 2013a,b,c,d,e, 2014b, 2015; Badawy and Jackson, 2012; Brodtmann et al., 1999). Another study of LICI in epilepsy, also using an intertrial interval of $5 \mathrm{~s}$ did not show a difference between the healthy control and drug naïve epilepsy groups (Silbert et al., 2015). Several studies have shown that the MEP amplitude is influenced by the intertrial interval but there are no studies assessing the influence of the intertrial interval on $\mathrm{LICl}$. The optimal intertrial interval to obtain reproducible single pulse MEPs is probably between 10-20 s (Julkunen et al., 2012; Möller et al., 2009; Pellicciari et al., 2016; Vaseghi et al., 2015), although other studies show that stimulus-response curves can be obtained reliably using shorter intertrial intervals (Mathias et al., 2014; Pearce et al., 2013). Other LICI studies, mostly in relatively small cohorts, used random intertrial intervals between 4$15 \mathrm{~s}$, but showed variability similar to our cohort (Kujirai et al., 1993; Sanger et al., 2001; Vallence et al., 2017; Valls-Solé et al., 1992; Wassermann et al., 1996). Interestingly, Center 2, with the shortest intertrial interval ( $1 \mathrm{~s}$ ) shows mean $\mathrm{LICl}>1$ at all ISIs, but median $\mathrm{LICl}<1$ at all ISIs, which may be due to several extreme outliers and speculatively a cumulative effect of the paired pulses. The relatively short intertrial intervals in all centers may thus have contributed to our inability to reproduce previous findings obtained with an ISI of 15 s (Badawy et al., 2010a, 2013a,d,e). A recent study, however, that also used an intertrial interval of $15 \mathrm{~s}$ showed facilitation at ISIs 50 , 80,110 and $140 \mathrm{~ms}$ in a group of 20 healthy volunteers (Bolden et al., 2017). Future studies are warranted to quantify the influence of intertrial interval on paired pulse TMS protocols.

Another explanation for the different results could be the participant cohorts. We only included data obtained from the dominant hemisphere in controls and generalized epilepsy and the ipsilateral hemisphere in focal epilepsy (i.e. the side of the seizure focus). In Center 4 it was standard practice to stimulate the dominant hemisphere only, leading to the exclusion of those in whom the dominant hemisphere was not the ipsilateral hemisphere. Including the results of both hemispheres in our multicenter analysis would have led to missing data for cases in which only one hemisphere was measured. Alternatively we could have estimated a mean of both hemispheres measured, which could have introduced a bias. Our choice lead to several exclusions, 
especially from Center 4, but we feel that this was the best way to deal with this issue. Even if some bias were introduced this way, we would expect the large differences between controls and subjects reported in other studies to have been visible in our large sample. We included a large number of subjects with all types of refractory generalized and focal epilepsy as well as healthy controls, while previous studies often report on relatively small samples ( 20 participants) of subjects with specific epileptic syndromes (Badawy et al., 2010a, 2012, 2013b,c,d, 2014b) or AEDs (Silbert et al., 2015). Our retrospective study design did not allow us to go into such detail, limiting the comparison with these studies. It should be noted that the participant cohorts reported in previous studies appear to overlap, potentially leading to multiple publication bias and overestimation of the consistency of these findings (Badawy et al., 2017; Bauer et al., 2017; Brigo et al., 2012).

Comparing TMS data across centers is challenging as variables such as the rMT depend directly on the equipment used. $\mathrm{LICl}$, however, is expressed as a response ratio, and thus is dimensionless. It is, therefore, theoretically better suited for comparison between centers than the rMT. We report on data collected from multiple centers and although it provides a large body of data, it is limited by its retrospective set-up and the different equipment and stimulation protocols used. Clear methodological guidelines for $\mathrm{LICl}$ stimulation are currently lacking, as there is insufficient data to define the most robust $\mathrm{LICl}$ protocol in terms of stimulator, stimulation intensity, coil type, stimulation site, target muscle, number of repetitions and intertrial interval. Some of the methodological differences are less likely to hamper the direct comparison between centers. For example, in three of the centers a figure-of-eight coil was used and stimulation was applied to the motor hotspot, while in one of the centers (Center 2) a circular coil was used and stimulation was applied on the vertex. The coil design (circular or figure-of-eight) affects the size and depth of the cortical region that is stimulated by the TMS pulse and may result in different $\mathrm{LICI}$ responses. While several studies compared different coils for shorter ISIs (Badawy et al., 2011; Cantello et al., 2000; Fleming et al., 2012; Shimizu et al., 1999), only one study directly compared these different coils for $\mathrm{LICl}$ in a small sample $(n=8)$ (Valzania et al., 1994). The LICI responses obtained at stimulation intensities of $110 \%$ and $120 \%$ rMT with either a circular or a figure-of-eight coil were similar in this study.

Other methodological differences are more likely to hamper direct comparison between centers. Centers 2 and 4 applied two pulses with an intensity of $110 \%$ rMT, while the other centers applied two pulses at $120 \%$ rMT. This was previously shown to yield small differences in the LICI response (Valls-Solé et al., 1992) and may have contributed to the weaker mean $\mathrm{LICl}(>1)$ seen in Center 2. Despite the difference in stimulation intensity used in Centers 3 and 4 , the mean $\mathrm{LICl}$ is $<1$ in both refractory 
epilepsy groups at ISIs 100, 150 and 200 ms, see Table 7.2, contrasting with previous studies showing facilitation at these ISIs in refractory epilepsy (Badawy et al., 2010a, $2013 a, d, e)$. In our refractory epilepsy groups, facilitation is only seen at $50 \mathrm{~ms}$ in genetic generalized epilepsy (Centers 3 and 4 ) and at $250 \mathrm{~ms}$ in focal epilepsy (Center 3). Centers 1 and 2 used biphasic pulses for stimulation. Biphasic and monophasic pulses preferentially excite partly different sets of cortical axons when using the same coil orientation (Groppa et al., 2012), potentially contributing to different response patterns. The number of repetitions and stimulation sequence (random or fixed) also differed, being relatively low in all centers, except in Center 1, where each ISI was repeated 50 times. Recent studies suggest the use of 20-30 repetitions for single pulse and short-interval paired pulse protocols (Chang et al., 2016; Goldsworthy et al., 2016), although 10 repetitions are commonly used in studies of epilepsy (Badawy et al., 2010b, 2012, 2013b,c,d, 2014b). All these methodological differences result in a net intercenter variability, which limits the direct comparison of $\mathrm{LICl}$ outcomes from different centers. We accounted for intercenter variability by using a linear mixedeffects model, and although this is a methodological robust solution, it cannot replace a prospective multicenter study in which all centers use exactly the same materials and methods. Despite the different methods, however, the results of Centers 1, 3 and 4 (and 2) are consistent, making the difference with the results of previous studies all the more striking. The individual studies that constituted our sample were set up as prospective TMS trials and part of the data was previously reported (Center 1: de Goede and van Putten (2017), Center 3: Pawley et al. (2017) and Center 4 (including patients with Dravet Syndrome): Stern et al. (2017)). In none of these studies were previous promising results replicated (Badawy et al., 2010a, 2013a,d,e), spurring the current retrospective multicenter analysis. Only one center (Center 3 ) in our study included all three subject types. Despite the same protocol and equipment being used, there was no significant difference between LICI measured in refractory generalized epilepsy, refractory focal epilepsy and control groups in this center, see also Pawley et al. (2017).

The $\mathrm{LICl}$ response ratios were not normally distributed in our sample. This is in line with one previous study of $\mathrm{LICl}$ (Silbert et al., 2015). Another study, reporting on a large cohort of healthy subjects, showed that rMT and short-interval intracortical inhibition do not follow a normal distribution (Wassermann, 2002). We suggest that statistical analyses of TMS variables should be done on log-transformed data. Furthermore, responses at different ISIs should be treated as repeated measurements, warranting corrections for multiple comparisons when several ISIs are measured in the same participants (Pawley et al., 2017).

Some authors consistently report low inter-individual variability of $\mathrm{LICl}$ (Badawy et al., 2010b, 2012, 2013a,b,d,e, 2014b, 2015; Badawy and Jackson, 2012); however, others 
reported much higher variability of TMS responses (Du et al., 2014; Lang et al., 2011; Nakamura et al., 1997; Sanger et al., 2001; Valls-Solé et al., 1992; Wassermann et al., 1996; Wassermann, 2002). In our cohort, the response ratio to long-interval paired pulse stimulation varied between individuals from strong inhibition to facilitation supporting some previous studies (Bolden et al., 2017; Valls-Solé et al., 1992). LICI variability was shown to be linked to the time of day and sleep status (Lang et al., 2011), neuropsychological profile (Bolden et al., 2017) and to age and hemispheric dominance (Vallence et al., 2017). While some TMS variables were shown to vary according to the menstrual cycle (Hattemer et al., 2007; Inghilleri et al., 2004; Smith et al., 2002), no data are available for $\mathrm{LICl}$. These and probably other unknown factors contribute to the large inter-individual variability of $\mathrm{LICl}$ and need to be adequately accounted for. One previous study accounted for the inter-individual variability by using a mixed model analysis, similar to our approach (Silbert et al., 2015).

An ideal biomarker for epilepsy diagnosis and management should show a difference in cortical excitability between drug naïve subjects with epilepsy and healthy controls. To be of clinical use for the management of epilepsy, it needs to show normalization of cortical excitability soon after treatment initiation in those who become seizurefree, but not in those with refractory seizures. In the latter group, cortical excitability should remain different from controls despite treatment indicating active epilepsy with (refractory) seizures. Our analysis (both with and without Center 2), however, shows the opposite effect. A good biomarker should also have low inter-individual variability, so that it can also be used to assess disease activity on an individual level. In our analysis, we only included subjects with refractory seizures and controls. Based on our findings showing both no significant differences between subjects with refractory epilepsy and controls, and a variable response to long-interval paired pulse stimulation, we argue that the use of $\mathrm{LICl}$ measured with TMS-EMG as a biomarker for epilepsy is limited and that a prospective trial is urgently needed to confirm this finding. Recent studies show that combining TMS with EEG may provide a more direct method to assess cortical excitability and underlying processes (Ilmoniemi et al., 1997; Ilmoniemi and Kičić, 2010). There is only one report of paired pulse TMS-EEG in epilepsy (Kimiskidis et al., 2017). Using feature selection methods combined with a Bayesian classifier, this study found a cross-validated diagnostic accuracy of 0.92 for differentiating genetic generalized epilepsy from healthy controls and 0.80 for differentiating responders from non-responders, suggesting that paired pulse TMS-EEG may be useful for diagnosis and the assessment of disease severity (Kimiskidis et al., 2017). Further research is needed to extend and confirm these findings. TMS-EEG may also help to reveal the mechanisms underlying the difference in $\mathrm{LICl}$ at ISIs 100 and $150 \mathrm{~ms}$ and $\mathrm{LICl}$ at ISIs 50, 200 and $250 \mathrm{~ms}$ that we found in all groups of our cohort. 


\subsection{Conclusion}

In this retrospective study, we could not replicate the difference in $\mathrm{LICl}$ measured with TMS-EMG between subjects with refractory genetic generalized and focal epilepsy and healthy controls consistently reported in previous studies. Methodological differences, especially shorter intertrial intervals, may have contributed to our inability to replicate previous findings. Further studies are needed to assess the influence of the length of the intertrial interval on $\mathrm{LICl}$ and to establish guidelines for $\mathrm{LICl}$ stimulation protocols. Based on our findings, $\mathrm{LICl}$ measured with TMS-EMG is unlikely to be useful as a biomarker in the clinical management of epilepsy. Future prospective multicenter trials are needed to confirm this finding.

\section{Acknowledgements}

The authors wish to thank the participants for their time.

\section{Funding}

This work was partly undertaken at UCLH/UCL Comprehensive Bio-Medical Research Center, which received a proportion of funding from the Department of Health's NIHR Biomedical Research Centers funding scheme. P.R.B. was supported by the Christelijke Vereniging voor de Verpleging van Lijders aan Epilepsie (Nederland). A.A.d.G. was supported by the Dutch TWIN foundation for neuromodulation. The work at King's College London (Center 3) was supported by an MRC Clinical Research Training Fellowship held by F.A.C. and a PhD Studentship funded by Epilepsy Action held by A.D.P. M.P.R. is funded in part by the NIHR Biomedical Research Center at the South London and Maudsley NHS Foundation Trust and by the UK Engineering and Physical Sciences Research Council (EPSRC) Center for Predictive Modelling in Healthcare at the University of Exeter (EP/N014391/1). The work at UCL (Center 4) was supported by the UK Epilepsy Society. J.W.S. receives research support from the Dr Marvin Weil Epilepsy Research Fund. The funding sources played no role in the design of the study, collection, analysis and interpretation of the data, and writing of the manuscript.

\section{Disclosures}

S.M.S. has been consulted by, or received fees for lectures, or institutional support, from GSK, Vitaflo, Nutricia, Eisai and UCB Pharma. M.J.A.M.v.P. is co-founder of Clinical Science Systems, the Netherlands. J.W.S. has been consulted by and received fees for lectures from GSK, Eisai and UCB Pharma. All other authors declare no conflicts of interest. 


\section{Supplementary material}

See online version of this article at https://doi.org/10.1093/brain/awx343 or scan the QR code.

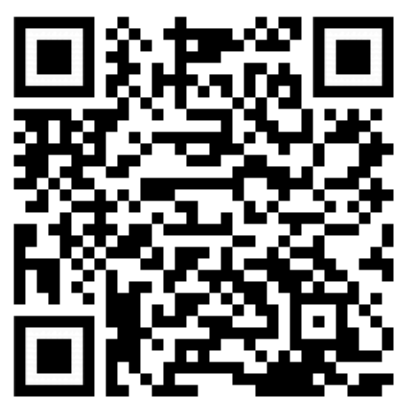





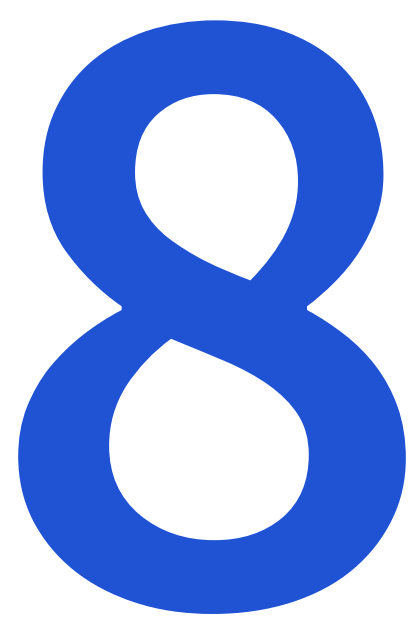

Multimodal TMS has the potential to improve the diagnostic process in epilepsy

A.A. de Goede and M.J.A.M. van Putten

In preparation 


\section{Abstract}

Objective: In epilepsy, a disturbed cortical excitability results in an enduring predisposition to generate seizures. In the diagnostic process, the electroencephalogram (EEG) is used to estimate the risk of seizure recurrence. However, the diagnostic sensitivity of the EEG remains limited, motivating the search for a novel biomarker. Transcranial magnetic stimulation (TMS) is a technique to assess cortical excitability. Combining the various TMS paradigms might enable diagnosis at the individual level. In this study we aim to evaluate differences between healthy controls, first seizure patients diagnosed with epilepsy and those without epilepsy, using a multimodal TMS approach.

Methods: We included twenty-one healthy subjects and twenty-one first seizure patients, of which seven were diagnosed with epilepsy. Both motor cortices were stimulated with 50 single pulses, followed by 50 paired pulses at interstimulus intervals (ISIs): 50, 100, 150, 200, 250 and 300 ms. Motor evoked potentials (MEPs) were recorded from the abductor digiti minimi muscle and TMS evoked potentials (TEPs) with a 64channel EEG. As single pulse TMS readouts we analyzed amplitudes of the MEP and TEP. For paired pulse stimulation, we assessed long intracortical inhibition ( $\mathrm{LICl})$ of the MEP and TEP.

Results: We found no significant differences in MEP or TEP amplitudes using single pulse TMS. Compared to healthy controls, epilepsy patients showed significantly increased $\mathrm{LICl}$ of the $\mathrm{N} 100$ and P180 components for ISI 200 ms. Furthermore, we found facilitation at ISI $100 \mathrm{~ms}$ in epilepsy patients and inhibition in patients without epilepsy.

Conclusion: Our findings indicate the potential of a multimodal TMS approach to improve the diagnostic process in epilepsy. At a group level, paired pulse TMS differentiated between healthy controls, patients with epilepsy and patients without epilepsy. To establish the true potential of multimodal TMS, data of more patients is needed, which may enable analysis at the individual level. Potential diagnostic biomarkers include increased LICI at ISI 200 ms and facilitation at ISI 100 ms. 


\subsection{Introduction}

Epilepsy is characterized by an enduring predisposition to generate epileptic seizures (Fisher et al., 2005, 2014), resulting from an imbalance between excitation and inhibition (McCormick and Contreras, 2001; McNamara, 1994). Although approximately $5 \%$ of the population will experience an unprovoked seizure during their lifetime (Forsgren et al., 1996; Hauser et al., 1993; Sander and Shorvon, 1996), less than half of them develop epilepsy (Berg, 2008; Bouma et al., 2016). Almost 70 million people are estimated to have epilepsy worldwide, making it one of the most common neurological diseases (Moshé et al., 2015; Ngugi et al., 2010, 2011).

Next to the clinical history, the electroencephalogram (EEG) is important in the diagnostic process. The tendency to generate seizures is reflected in the EEG by the presence of interictal epileptiform discharges (IEDs) (Koutroumanidis et al., 2017; Krumholz et al., 2007; Rosenow et al., 2015), reflecting abnormal cortical excitability. However, IEDs are not necessarily found which limits the diagnostic sensitivity of the routine EEG (Fisher et al., 2005). A meta-analysis estimated the sensitivity at $17.3 \%$ (specificity $94.7 \%$ ) in adults and the probability of epilepsy in case of a normal routine EEG at 47\% (Bouma et al., 2016). Sensitivity can be moderately increased by performing additional EEG recordings, such as after sleep deprivation or a 24 hour recording (Geut et al., 2017; King et al., 1998; Rosenow et al., 2015). Repeated and prolonged recordings can increase the diagnostic yield to approximately 60-90\% (Rosenow et al., 2015), with a significant increase in review time. Furthermore, IEDs are absent in approximately $10 \%$ of the diagnosed epilepsy patients (Rosenow et al., 2015). This motivates the search for other biomarkers for epilepsy.

Transcranial magnetic stimulation (TMS) is a non-invasive technique to assess cortical excitability (Barker et al., 1985). Whereas single pulse TMS in combination with electromyography (EMG) measures the integrated corticospinal excitability (Abbruzzese and Trompetto, 2002; Valls-Solé et al., 1992), paired pulse TMS-EMG provides information about the relative contribution of excitatory and inhibitory cortical networks (Kujirai et al., 1993; Valls-Solé et al., 1992; Ziemann et al., 1996a). TMS-EMG studies found significantly increased excitability in drug naïve new-onset epilepsy patients compared to healthy controls, being the most consistent for the paired pulse TMS paradigm (de Goede et al., 2016). Instead of inhibition, facilitation is found at short (2 and 5 $\mathrm{ms}$ ) and long (250 and $300 \mathrm{~ms}$ ) interstimulus intervals (ISIs) for both hemispheres in idiopathic generalized epilepsy and for the hemisphere ipsilateral to the epileptic focus in focal epilepsy (Badawy et al., 2007, 2013a; de Goede et al., 2016). Although not as prominent as in new-onset epilepsy patients, increased excitability at ISIs 250 and $300 \mathrm{~ms}$ is also found in patients with isolated unprovoked seizures without 
recurrence (Badawy et al., 2014b). The ability to differentiate between healthy subjects and patients presenting with a first seizure who develop epilepsy and those who do not, would largely improve the diagnostic process in epilepsy. However, significant differences have only been reported on a group level, while diagnosis at the level of an individual patient is desirable. The combination of TMS and EEG enables measurement of the direct brain response (Ilmoniemi et al., 1997; Ilmoniemi and Kičić, 2010). We are not aware of TMS-EEG studies in drug naïve epilepsy patients. In this study, we use single and paired pulse TMS combined with EMG and EEG. Such a multimodal TMS approach might improve the diagnostic process in epilepsy.

This study aims to evaluate differences in cortical excitability between healthy controls, first seizure patients diagnosed with epilepsy and those without epilepsy, using a multimodal TMS approach. As outcome measures we analyze the resting motor threshold (rMT), motor evoked potential (MEP) amplitude, long intracortical inhibition (LICl) and TMS evoked potential (TEP).

\subsection{Materials and methods}

The study protocol (trial ID: NL49854.044.14) was approved by the local medical ethics committee (Medisch Spectrum Twente, Enschede, the Netherlands). We performed this study in accordance with the Declaration of Helsinki and the guidelines for the use of TMS in clinical practice and research (Rossi et al., 2009). Data of healthy subjects was used previously in another context by de Goede and van Putten (2017), de Goede et al. (2018) and Bauer et al. (2018).

\subsubsection{Subjects}

Patients visiting the neurology or clinical neurophysiology department of the Medisch Spectrum Twente (Enschede, the Netherlands) with the presentation of a first seizure were approached to participate. Healthy controls were recruited by posting flyers. All first seizure patients and healthy subjects (18 years or older) gave written informed consent. Subjects were excluded in the case of contraindications to TMS as described in the Screening Questionnaire before TMS (Rossi et al., 2011), except for one seizure in patients. In addition, patients needed to be naïve to anti-epileptic drugs (AEDs).

The epilepsy diagnosis was made by the treating neurologist, based on the clinical history combined with the EEG and when necessary magnetic resonance imaging (MRI) results. A patient had epilepsy in case of 1 ) at least two unprovoked seizures more than 24 hours apart, or 2) one unprovoked seizure with an increased risk of recurrence, or 3 ) a diagnosed epilepsy syndrome (Fisher et al., 2005, 2014). A diagnosis of generalized epilepsy required generalized IEDs on the EEG and/or a clear history of 
either absences, myoclonic, atonic, tonic or tonic-clonic seizures. For a diagnosis of focal epilepsy, it was required that seizure symptomatology and/or the EEG showed prominent and consistent lateralization to one hemisphere (Scheffer et al., 2017). First seizure patients with no seizure recurrence for at least one year and no IEDs on the routine (and additional) EEG(s) were not diagnosed with epilepsy.

We estimated that we needed to include at least 20 healthy controls, 20 first seizure patients without epilepsy, 20 generalized epilepsy patients and 20 focal epilepsy patients to evaluate the potential of multimodal TMS to improve the diagnostic process in epilepsy.

\subsubsection{Stimulation protocol}

Both motor hotspots were stimulated using biphasic pulses with a duration of 400 $\mu \mathrm{s}$, applied by a $70 \mathrm{~mm}$ figure-of-eight air film coil connected to a Magstim Rapid ${ }^{2}$ stimulator (The Magstim Company Ltd, Whitland, United Kingdom). The coil was manually positioned and held at the hotspot of the abductor digiti minimi (ADM) muscle. It was placed tangentially, with the handle pointing backwards and laterally at an angle of $45^{\circ}$ away from the midline. We applied 50 single pulses, followed by 50 paired pulses at each of the six randomly applied ISIs: 50, 100, 150, 200, 250 and $300 \mathrm{~ms}$. An interval varying from 3.5-4.5 s was kept between single pulses and pairs of paired pulses. All pulses were given at a stimulation intensity of $120 \%$ rMT, with rMT defined as the minimum intensity needed to evoke five or more MEPs, with an amplitude larger than $50 \mu \mathrm{V}$, out of ten consecutive pulses (Groppa et al., 2012; Rossini et al., 2015).

Subjects were asked to keep their head in a fixed position during stimulation, their eyes open and their hands pronated and relaxed. In addition, they listened to adaptive noise played at a maximum intensity of $95 \mathrm{~dB}$ to mask the clicking sound of the TMS pulses.

\subsubsection{EMG recording and analysis}

To measure EMG responses, surface electrodes were placed in a belly-tendon montage over the ADM muscle contralateral to the stimulated hemisphere. We continuously recorded the EMG, using the bipolar channel on the 64-channel EEG amplifier (TMSi, Oldenzaal, the Netherlands). The EMG signal was sampled at $5000 \mathrm{~Hz}$ and low-pass filtered with an anti-aliasing filter with a cut-off frequency of $1000 \mathrm{~Hz}$.

Even though we instructed subjects to fully relax their hand, EMG recordings were afterwards checked for muscle pre-activation. Responses were excluded when muscle activity was larger than $50 \mu \mathrm{V}$ in the $50 \mathrm{~ms}$ prior to the single or conditioning pulse for single and paired pulse TMS, respectively.

As single pulse TMS-EMG measure we evaluated the MEP amplitude. For each subject, 
the peak-to-peak amplitudes of the EMG responses were calculated and averaged over the 50 single pulses.

As paired pulse TMS-EMG measure we determined $\mathrm{LICl}$. For each subject and each ISI, we calculated the mean peak-to-peak amplitude of the responses to the 50 conditioning and test pulses. Thereafter, the ratio was calculated between the mean test response (TR) amplitude and mean conditioning response (CR) amplitude, expressed as a percentage: $100 \cdot$ TR/CR (\%). Values below 100\% represent inhibition and values above $100 \%$ facilitation.

\subsubsection{EEG recording and analysis}

The EEG was continuously recorded using a TMS-compatible EEG cap (ANT Neuro, Enschede, the Netherlands) connected to a 64-channel EEG amplifier (TMSi, Oldenzaal, the Netherlands) and the NeuroCenter EEG software (Clinical Science Systems, Leiden, the Netherlands). The ground electrode was located between electrode positions $\mathrm{Fz}$ and Fpz. The EEG signal was sampled at $5000 \mathrm{~Hz}$ and low-pass filtered with an antialiasing filter with a cut-off frequency of $1000 \mathrm{~Hz}$.

The EEG was analyzed with the common average reference and down sampled to a frequency of $1000 \mathrm{~Hz}$. Each EEG response was baseline corrected by subtracting the mean EEG signal measured over a period of 350-100 ms prior to the single or conditioning pulse for single and paired pulse TMS, respectively. Trials were defined from 50 ms before till $650 \mathrm{~ms}$ after each single or conditioning pulse. We applied single trial principal component analysis (PCA) to reduce the TMS artifacts caused by the magnetic pulse and muscle activation on the scalp. For a detailed description of the PCA method, see ter Braack et al. (2013a). After removing the first four out of forty principal components, trials were filtered between $1-45 \mathrm{~Hz}$ using a fourth order Butterworth bandpass filter.

As single pulse TMS-EEG measure we evaluated the single pulse TEP. For each subject, the average was calculated over the 50 single pulse EEG trials per electrode position.

As paired pulse TMS-EEG measure we determined LICI. Unlike the MEP, the TEP is still ongoing when giving the test pulse, making $\mathrm{LICl}$ analysis more complicated. Per subject and ISI, the uncorrected paired pulse TEPs were obtained by taking the average over the 50 paired pulse EEG trials. However, the late components of the response evoked by the conditioning pulse are likely affecting the early components of the response evoked by the test pulse. Simultaneously, these same late components of the conditioning response are likely affected by giving the test pulse. Since it is impossible to address both interactions due to their entanglement, we applied a correction method that only focuses on the influence of the late conditioning response on the early test response 
(Daskalakis et al., 2008; Premoli et al., 2014b). The paired pulse TEP was obtained by subtracting the single pulse TEP from the uncorrected paired pulse TEP. Alignment of the single and test pulse, enabled direct comparison of the single and paired pulse TEP. To assess $\mathrm{LICl}$, we again subtracted the single pulse TEP from the paired pulse TEP (Premoli et al., 2014b).

\subsubsection{Statistical analysis}

In this study, TMS-EMG-EEG measures were obtained from both hemispheres, although occasionally it was only possible to stimulate one side. Statistical analysis were performed separately for the dominant (or ipsilateral) and non-dominant (or contralateral) hemisphere, as scored by the Dutch Handedness Questionnaire (van Strien, 1992, 2003). Instead of hand dominance, seizure lateralization was used in patients diagnosed with focal epilepsy. Throughout the statistical analysis, each ISI was analyzed individually for paired pulse TMS measures.

To evaluate the TMS-EMG measures, we used one-way ANOVA to test if there were significant differences in rMT, mean MEP amplitude and $\mathrm{LICl}$ between healthy controls, patients with epilepsy and patients without epilepsy. In case of significance, post hoc tests were performed to identify which subject categories differed from each other.

To evaluate the TMS-EEG measures, the cluster-based permutation analysis (Maris and Oostenveld, 2007) was applied as implemented in FieldTrip (http://www.fieldtriptool box.org). To compensate for handedness and seizure lateralization, we mirrored topographical maps of left-handed subjects and epilepsy patients with a focal onset zone in their non-dominant hemisphere (e.g. responses of electrode positions C3 and C4 were exchanged). Independent samples $t$-tests were used to compare the single pulse TEPs between healthy controls and epilepsy patients. These comparisons were done per electrode position and time sample over a period of $300 \mathrm{~ms}$ after the single pulse. In addition, we evaluated each of the characteristic TEP components individually (P30: interval 15-40 ms, N45: interval 40-55 ms, P60: interval 55-75 $\mathrm{ms}, \mathrm{N} 100$ : interval $75-140 \mathrm{~ms}$ and P180: interval $140-250 \mathrm{~ms})$. Only $t$-values with a $p$-value $<0.05$ were clustered based on adjacent time samples and neighboring electrodes $(n \geq 2)$. To determine significance, we statistically tested the summed $t$ value of each cluster against the distribution obtained by permutation using the Monte Carlo method. Here, single pulse TEPs were randomly assigned to either a healthy subject or an epilepsy patient for 1500 times. We assumed clusters to be significant if less than $5 \%$ of the summed $t$-values obtained by permutation exceeded the original cluster $t$-value, i.e. if $p$-value $<0.05$. The same analysis was performed to compare the single pulse TEPs between healthy controls and patients without epilepsy, as well as between patients with and without epilepsy. In addition, it was used to test if there 
Table 8.1: Characteristics of the first seizure patients, including abnormalities found on the EEG (intermittent findings) and MRI.

\begin{tabular}{|c|c|c|c|c|c|}
\hline \# Sex & Age & Epilepsy type & EEG abnormalities & MRI abnormalities & Notes \\
\hline $01 \mathrm{~m}$ & 38 & - & No & No & Not included in analysis \\
\hline $02 \mathrm{~m}$ & 24 & - & No & No & \\
\hline $03 \mathrm{~m}$ & 58 & - & No & No & \\
\hline $04 \mathrm{~m}$ & 18 & - & No & No & \\
\hline $05 \mathrm{f}$ & 69 & - & Delta waves L: FT & No & Not included in analysis \\
\hline $06 \mathrm{~m}$ & 62 & Focal & IEDs R: FT & Lesion R: T, likely DNET & \\
\hline $07 \mathrm{~m}$ & 47 & - & No & CM type 1 & \\
\hline $08 \mathrm{~m}$ & 46 & - & No & No & \\
\hline $09 \mathrm{~m}$ & 56 & - & No & No & \\
\hline $10 \mathrm{~m}$ & 51 & - & No & $\mathrm{CT}(\mathrm{A}): \mathrm{No}^{*}$ & \\
\hline $11 \mathrm{f}$ & 19 & - & No & Focal dysplasia L: T & \\
\hline $12 \mathrm{f}$ & 23 & Focal & IEDs L: T & No & \\
\hline $13 \mathrm{~m}$ & 19 & - & No & Contusion L: F, R: T & Symptomatic insult \\
\hline $14 \mathrm{f}$ & 61 & - & Delta waves R: T & Old lacunar infarcts $R+L$ & \\
\hline $15 \mathrm{~m}$ & 56 & - & Delta waves $\mathrm{R}>\mathrm{L}: \mathrm{T}$ & (sub)acute ischemia R: PO & Symptomatic insult \\
\hline $16 \mathrm{~m}$ & 58 & - & No & No & \\
\hline $17 \mathrm{~m}$ & 53 & Focal & IEDs R + L: T & No & \\
\hline $18 \mathrm{~m}$ & 25 & Generalized & $\begin{array}{l}\text { Delta \& theta waves } \\
\qquad(\mathrm{L}>\mathrm{R}: \mathrm{T})\end{array}$ & No & \\
\hline $19 \mathrm{~m}$ & 58 & Generalized & No & No & \\
\hline $20 \mathrm{f}$ & 23 & - & No & No & \\
\hline $21 \mathrm{~m}$ & 24 & - & No & Small infarct $R: T$ & \\
\hline $22 \mathrm{f}$ & 48 & Generalized & No & No & Also PNES diagnosis \\
\hline $23 \mathrm{~m}$ & 59 & Focal & IEDs R: FT & No & \\
\hline
\end{tabular}

$\mathrm{m}=$ male; $\mathrm{f}=$ female; $\mathrm{R}=$ right $\mathrm{L}$ = left $\mathrm{F}$ = frontal; $\mathrm{T}$ = temporal; $\mathrm{P}=$ parietal $\mathrm{O}$ = occipital; $\mathrm{DNET}$ = dysembryoplastic neuroepithelial tumor; $\mathrm{CM}=$ Chiari malformation; $\mathrm{PNES}=$ psychogenic non-epileptic seizure. ${ }^{*}$ MRI not possible due to pacemaker, instead CT cerebrum and CTA.

were significant differences in LICl between healthy controls, patients with epilepsy and patients without epilepsy. Because each group was compared with each other, resulting in three comparisons per measure, we applied the Bonferroni correction ( $\mathrm{n}=$ $3, p$-value $<0.017$ ) to adjust for multiple testing.

Matlab (version R2015a, The Mathworks, Natick, MA, USA) was used for data analysis. If not stated otherwise, we considered a $p$-value $<0.05$ to be statistically significant.

\subsection{Results}

Twenty-one first seizure patients (16 males, mean age $42.3 \pm 17$ years; range 1862 years, 18 right-handed) were included in these analyses. Seven patients were diagnosed with epilepsy, four with focal epilepsy and three with generalized epilepsy, see Table 8.1. Two additional first seizure patients were included in the study, but 
Table 8.2: Group average (mean \pm standard deviation (SD)) of rMT values for each subject category, with group size indicated between brackets. rMT intensity is expressed as the percentage of maximum stimulator output $(0.8 \mathrm{~T})$.

\begin{tabular}{lcc}
\hline Subject category & $\begin{array}{c}\text { Dominant hemisphere } \\
\text { mean rMT } \pm \text { SD }(\%)\end{array}$ & $\begin{array}{c}\text { Non-dominant hemisphere }^{2} \\
\text { mean rMT } \pm \text { SD (\%) }\end{array}$ \\
\hline Healthy controls $(\boldsymbol{n}=\mathbf{2 1})$ & $68.2 \pm 8.4$ & $66.5 \pm 8.0$ \\
Patients with epilepsy $(\boldsymbol{n}=\mathbf{7})$ & $76.3 \pm 8.5$ & $76.4 \pm 4.1$ \\
Patients without epilepsy $(\boldsymbol{n}=\mathbf{1 4})$ & $73.5 \pm 12.9$ & $68.5 \pm 12.4$ \\
\hline
\end{tabular}

${ }^{1}$ or ipsilateral hemisphere; ${ }^{2}$ or contralateral hemisphere.

not in the statistical analyses. In one patient MEPs were often not evoked, while data was not stored correctly for the other. Furthermore, data of twenty-one healthy controls (6 males, mean age $28.6 \pm 8$ years; range $20-49$ years, 19 right-handed) was included. Thirteen out of the originally included thirty-four controls were left out of these analyses. In ten subjects robot-guided coil positioning was applied, in two subjects stimulation at $120 \%$ rMT was impossible due to a high rMT and one subject did not completed the TMS session after not feeling well.

\subsubsection{Single pulse TMS-EMG: rMT}

We found the lowest rMT values in healthy controls and the highest in epilepsy patients, see Table 8.2. No significant differences were found between healthy controls, patients with epilepsy and patients without epilepsy (dominant hemisphere: $F(2,36)=2.0, p=$ 0.15 ; non-dominant hemisphere: $F(2,34)=3.2, p=0.052$ ).

\subsubsection{Single pulse TMS-EMG: MEP amplitude}

When comparing healthy controls, patients with epilepsy and patients without epilepsy to each other, we found no significant differences in mean MEP amplitude (dominant hemisphere: $F(2,35)=1.6, p=0.21$; non-dominant hemisphere: $F(2,34)=0.03, p=0.97$, see Table 8.3.

\subsubsection{Paired pulse TMS-EMG: LICI}

Instead of inhibition, we found facilitation in epilepsy patients for ISI $100 \mathrm{~ms}$ when stimulating the dominant side (dominant hemisphere: $F(2,35)=3.6, p=0.04$; nondominant hemisphere: $F(2,34)=0.5, p=0.62$ ), see Figure 8.1. Post hoc tests identified a significant difference in $\mathrm{LICl}$ between patients with and without epilepsy, but not between epilepsy patients and healthy controls. Also for ISI $200 \mathrm{~ms}$, we found a significant difference in $\mathrm{LICl}$ between the three subject categories when stimulating the dominant side (dominant hemisphere: $F(2,35)=3.5, p=0.04$; non-dominant 
Table 8.3: Group average (mean \pm SD) of MEP amplitude for each subject category, with group size indicated between brackets.

\begin{tabular}{lcc}
\hline Subject category & $\begin{array}{c}\text { Dominant hemisphere } \\
\text { mean MEP ampl } \pm \text { SD }(\mathrm{mV})\end{array}$ & $\begin{array}{c}\text { Non-dominant hemisphere }^{2} \\
\text { mean MEP ampl } \pm \text { SD }(\mathrm{mV})\end{array}$ \\
\hline Healthy controls $(\boldsymbol{n}=\mathbf{2 1})$ & $1.3 \pm 1.1$ & $1.4 \pm 0.8$ \\
Patients with epilepsy $(\boldsymbol{n}=\mathbf{7})$ & $1.9 \pm 1.5$ & $1.4 \pm 1.4$ \\
Patients without epilepsy $(\boldsymbol{n}=\mathbf{1 4})$ & $1.0 \pm 0.6$ & $1.3 \pm 1.1$ \\
\hline
\end{tabular}

${ }^{1}$ or ipsilateral hemisphere; ${ }^{2}$ or contralateral hemisphere.

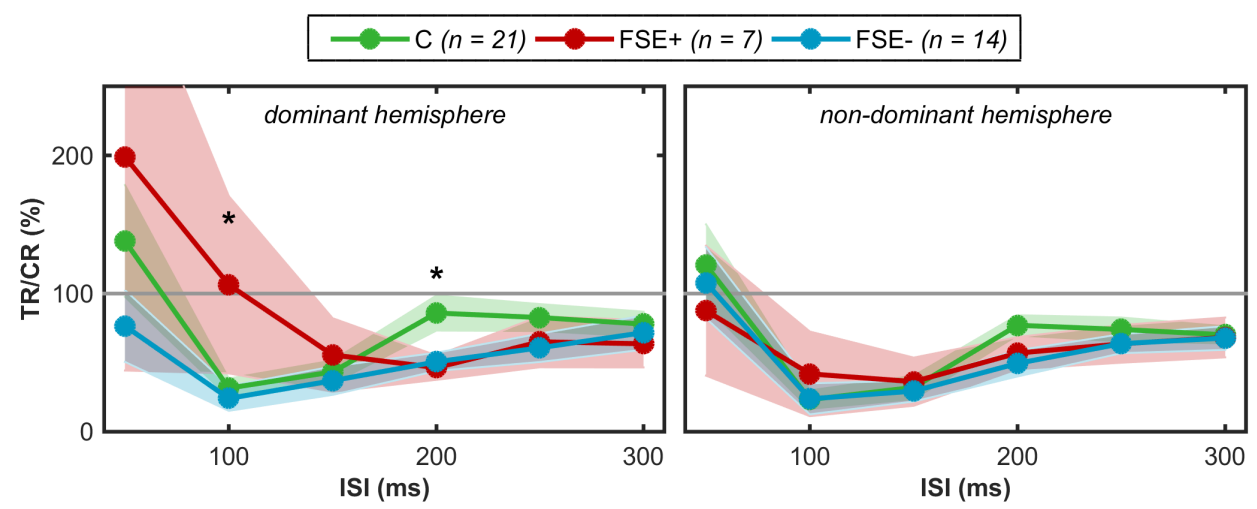

Figure 8.1: Group average (mean \pm standard error of the mean (SEM)) of LICl in healthy controls ( $C$ in green), first seizure patients diagnosed with epilepsy (FSE + in red) and first seizure patients without epilepsy (FSE- in blue). We stimulated both the dominant (or ipsilateral) hemisphere (on the left) and the non-dominant (or contralateral) hemisphere (on the right). TR = test response, $\mathrm{CR}=$ conditioning response and ISI = interstimulus interval. We found a significant difference in $\mathrm{LICl}$ between the three subject categories for ISIs 100 and $200 \mathrm{~ms}$ when stimulating the dominant hemisphere, represented by the asterisk.

hemisphere: $F(2,34)=2.9, p=0.07)$. Despite a trend for increased LICI in both patients with and without epilepsy compared to healthy controls, post hoc tests did not identify subject categories that differed significantly. No significant differences were found for the other ISIs (dominant hemisphere: $F(2,35)=0.8,0.4,1.2,0.3$ and $p=0.44,0.67,0.31$, 0.75 ; non-dominant hemisphere: $F(2,34)=0.2,0.07,0.4,0.02$ and $p=0.82,0.93,0.67$, 0.98 ; for ISIs 50, 150, 250 and $300 \mathrm{~ms}$, respectively).

\subsubsection{Single pulse TMS-EEG: TEP}

The single pulse TEPs did not differ significantly between healthy controls, patients with epilepsy and patients without epilepsy, see Figure 8.2. We found no significant clusters when comparing the three subject categories. 

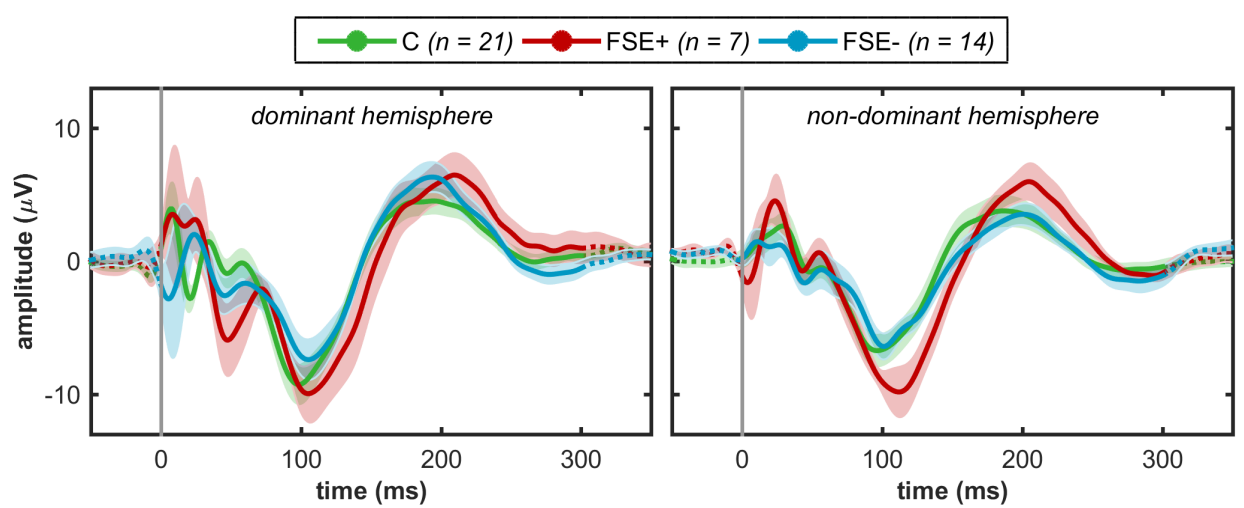

Figure 8.2: Group average (mean \pm SEM) of single pulse TEP in healthy controls ( $C$ in green), first seizure patients diagnosed with epilepsy (FSE+ in red) and first seizure patients without epilepsy (FSE- in blue). We stimulated both the dominant (or ipsilateral) hemisphere (on the left) and the non-dominant (or contralateral) hemisphere (on the right). The TEP is the response at electrode position $\mathrm{Cz}$. No significant differences were found between the subject categories.

\subsubsection{Paired pulse TMS-EEG: LICI}

Before evaluating the paired pulse TEP, we applied a method to correct for the influence of the late conditioning response on the early test response (Daskalakis et al., 2008; Premoli et al., 2014b), see Figure 8.3. To assess LICl, the single pulse TEP was subtracted from the paired pulse TEP (Premoli et al., 2014b). Figure 8.3 indicates that $\mathrm{LICl}$ is characterized by strong inhibition of mainly the late paired pulse TEP components. Inhibition of the N100 is reflected by positive values around $100 \mathrm{~ms}$ and inhibition of the P180 by negative values around $200 \mathrm{~ms}$, where a larger value corresponds to stronger inhibition.

Compared to healthy controls, epilepsy patients showed significantly increased $\mathrm{LICl}$ of the $\mathrm{N} 100$ and P180 components for ISI 200 ms when stimulating the dominant hemisphere, see Figure 8.4. We found a significant negative N100 cluster $(p<0.001)$ centrally on the contralateral side and a significant positive P180 cluster $(p=0.003)$ centro-frontally on the ipsilateral side. When stimulating the non-dominant hemisphere, we found increased $\mathrm{LICl}$ of the P30 component in patients with epilepsy compared to patients without epilepsy for ISI $100 \mathrm{~ms}$. A significant positive P30 cluster $(p=0.002)$ was found parieto-occipitally on the ipsilateral side. Finally, we found a significant difference in $\mathrm{LICl}$ between healthy controls and epilepsy patients for ISI 150 ms (positive N45 cluster, $p=0.003$ ). Only the N100 and P180 clusters at ISI 200 ms were found when evaluating the entire period of $300 \mathrm{~ms}$. The two other clusters were only detected when focusing on the individual TEP components. 

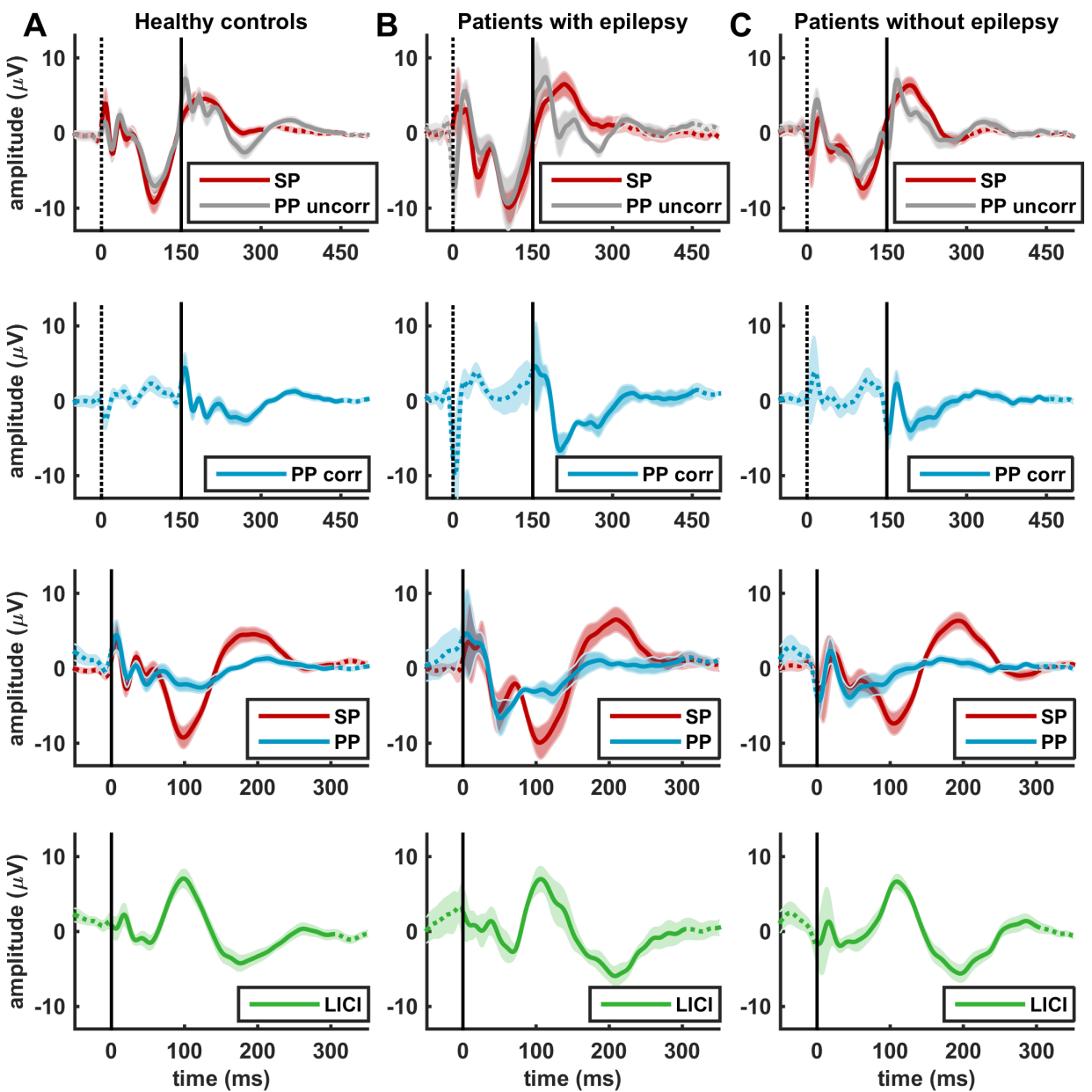

Figure 8.3: Representation of the paired pulse correction method used to correct for the influence of the late conditioning response on the early test response for ISI $150 \mathrm{~ms}$. The corrected paired pulse TEP (PP corr or PP in blue) is obtained by subtracting the single pulse TEP (SP in red) from the uncorrected paired pulse TEP (PP uncorr in grey). Alignment of the single pulse (black dotted line) and test pulse (black solid line) enabled subtraction of the single pulse TEP from the paired pulse TEP to assess $\mathrm{LICl}$ (in green). Each TEP and $\mathrm{LICl}$ curve is the average (mean \pm SEM) at electrode position $\mathrm{C} z$ when stimulating the dominant (or ipsilateral) hemisphere in A) healthy controls, B) first seizure patients diagnosed with epilepsy or C) first seizure patients without epilepsy. 

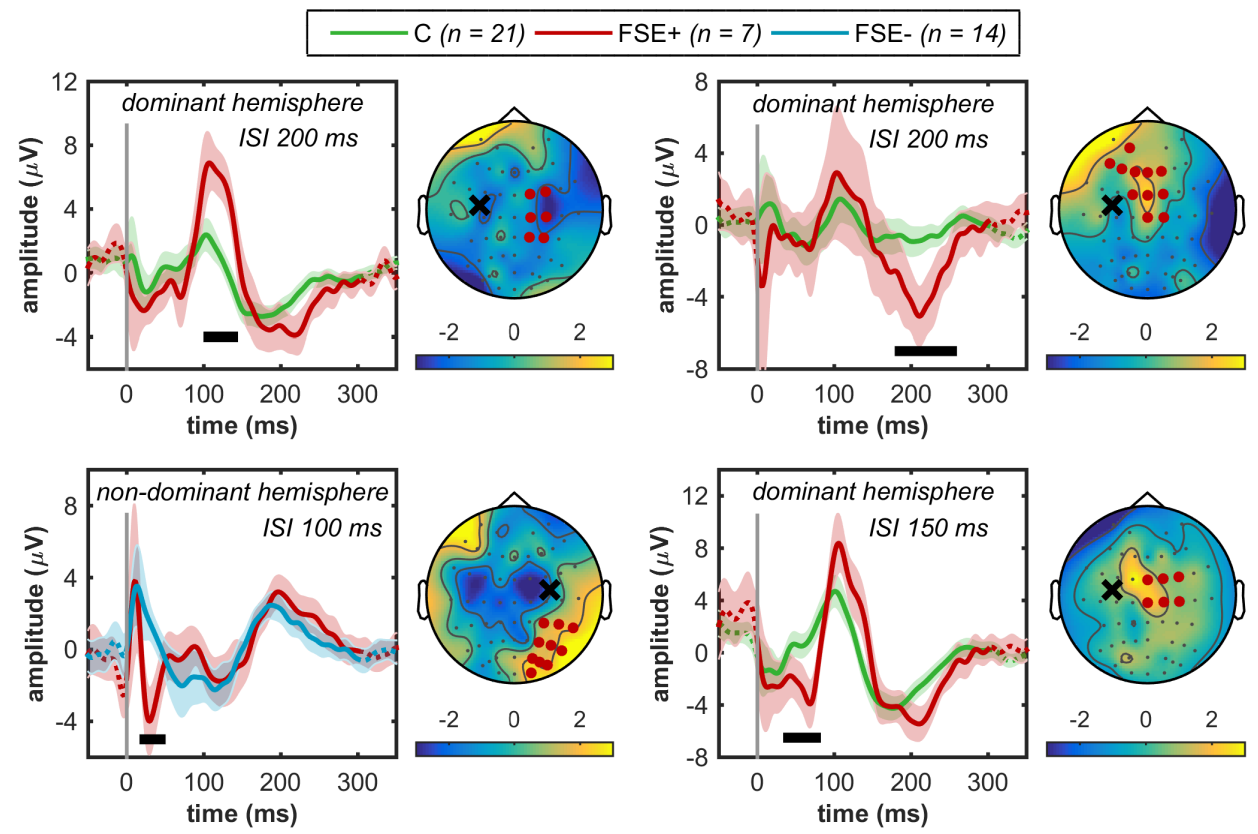

Figure 8.4: Overview of significant clusters when comparing $\mathrm{LICl}$ between healthy controls (C in green), first seizure patients diagnosed with epilepsy (FSE+ in red) and first seizure patients without epilepsy (FSE- in blue). For each cluster the group averaged (mean \pm SEM) LICl over all significant electrodes is presented, as well as the difference in topographical $\mathrm{LICl}$ distribution at the time of the cluster. The black cross indicates the stimulation site and the red dots significant electrodes. The thick bar in each subpanel represents the time window of significant differences in $\mathrm{LICl}$. Yellow areas indicate increased inhibition of positive TEP amplitudes in first seizure patients and blue areas increased inhibition of negative TEP amplitudes.

\subsection{Discussion}

In this study we evaluated the potential of multimodal TMS to improve the diagnostic process in epilepsy. Single pulse TMS could not differentiate between healthy controls, first seizure patients diagnosed with epilepsy and those without epilepsy. However, we found promising results for paired pulse TMS of the dominant hemisphere. In general, cortical excitability was decreased in epilepsy patients, represented by increased $\mathrm{LICI}$ and a trend towards a higher rMT.

Compared to healthy controls, epilepsy patients showed significantly increased LICI of the N100 and P180 components for ISI 200 ms. Using paired pulse TMS-EMG, we also found a significant difference in LICI between the three subject categories for ISI $200 \mathrm{~ms}$. Both patients with and without epilepsy showed non-significant increased $\mathrm{LICl}$ compared to healthy controls. Furthermore, we found facilitation at ISI $100 \mathrm{~ms}$ in diagnosed 
epilepsy patients instead of inhibition as in patients without epilepsy.

Our findings indicate the potential of increased $\mathrm{LICl}$ at ISI $200 \mathrm{~ms}$ and facilitation at ISI 100 ms as diagnostic paired pulse TMS-EMG biomarkers. In contrast, previous TMS-EMG studies mainly reported significant differences between drug naïve newonset epilepsy patients and healthy controls for ISIs 250 and 300 ms (de Goede et al., 2016). Additionally, differences between new-onset epilepsy patients and patients with isolated seizures without recurrence were also found for ISIs 250 and $300 \mathrm{~ms}$ (Badawy et al., 2014b). However, the majority of these studies were performed by Badawy and colleagues. Even though they appear to confirm their findings repeatedly for different types of epilepsy, studies were less independent than suggested (Badawy et al., 2017; Bauer et al., 2017). Data of healthy controls was frequently reused (Badawy et al., 2013b,d,e, 2014b, 2015, 2017), while data of patients with generalized epilepsy (Badawy et al., 2013b,d, 2014b, 2017) or focal epilepsy (Badawy et al., 2013b,e, 2014b, 2015,2017 ) were partially reused. Instead of new-onset epilepsy, one recent study evaluated drug naïve generalized epilepsy patients that were already diagnosed for months to years (Silbert et al., 2015). Even though they reported the largest differences with healthy controls for ISIs $200-250 \mathrm{~ms}$, LICI did not increased significantly. Two other recent studies focused on patients with active epilepsy despite the use of AEDs. Poorly controlled patients showed significantly increased $\mathrm{LICl}$ compared to healthy subjects for ISIs 50 and $200 \mathrm{~ms}$ (Pawley et al., 2017). After correction for multiple comparisons, however, these outcomes were no longer significant. A large multicenter study found significant changes in LICI for refractory focal epilepsy for ISIs 100 and 200 $\mathrm{ms}$, but not for refractory generalized epilepsy (Bauer et al., 2018). Especially the latest study suggests, in line with our findings, that $\mathrm{LICl}$ at ISIs 100 and $200 \mathrm{~ms}$ might be a biomarker for (active) epilepsy. Furthermore, these three recent studies all tended to find decreased cortical excitability in epilepsy patients.

Potential diagnostic paired pulse TMS-EEG biomarkers include increased $\mathrm{LICl}$ of the $\mathrm{N} 100$ and P180 components for ISI $200 \mathrm{~ms}$. Of all significant clusters, we found the most consistent results for these late TEP components. Both clusters were detected when evaluating the entire period of $300 \mathrm{~ms}$ as well as when focusing on the individual TEP components. In epilepsy patients treated with AEDs, single pulse TMS also revealed differences for the N100 and P180 components in particular (ter Braack et al., 2016; Del Felice et al., 2011; Julkunen et al., 2013). Since AEDs can significantly affect cortical excitability (Ziemann et al., 2015), it is unclear to what extent these differences are related to epilepsy or to the use of AEDs. Nevertheless, the N100 and P180 components seem to have the greatest potential to become a biomarker for epilepsy. Other factors in favor of these late components include that they are usually not affected by artifacts and that they have the largest amplitude and highest reliability of all TEP components 
(Kerwin et al., 2018). For ISI 100 ms, we found increased LICI of the P30 component in patients with epilepsy compared to patients without epilepsy when stimulating the non-dominant hemisphere. For the dominant side, we found facilitation in epilepsy patients and inhibition in patients without epilepsy. Although the P30 component itself is associated with excitatory processes (Mäki and Ilmoniemi, 2010b), LICl of the P30 is thought to reflect cortical inhibition (Opie et al., 2017; Rogasch et al., 2013).

We found decreased cortical excitability in epilepsy patients, except for facilitation at ISI 100 ms. Epilepsy is commonly associated with hyperexcitability of cortical networks, resulting from an imbalance between excitatory and inhibitory activity (McCormick and Contreras, 2001; McNamara, 1994). However, cortical excitability appears to fluctuate depending on the disease state. Although hyperexcitability was indeed found for 24 hours prior to a seizure (Badawy et al., 2008), excitability was significantly decreased for 24-48 hours following a seizure (Badawy et al., 2008; Delvaux et al., 2001). In addition, cortical excitability was still increased in drug naïve new-onset epilepsy patients within 1-2 weeks after their first seizure (Badawy et al., 2013a). Whereas hyperactivation of inhibitory neuronal circuits was observed when drug naïve patients were measured months or years later (Lee et al., 2015; Silbert et al., 2015). In both situations, the decrease in cortical excitability is thought to act as a protective mechanism to prevent seizure recurrence (Badawy et al., 2008; Delvaux et al., 2001; Lee et al., 2015). Even though we tried to measure patients as soon as possible after their first seizure, this usually took longer than a month (mean $38 \pm 26$ days). We speculate that the decreased excitability may result from compensatory mechanisms to prevent new seizures. However, the influence of elapsed time since the initial seizure on the cortical excitability needs to be further investigated. For the clinical applicability of TMS in the diagnostic process, it is essential to know whether excitability indeed reverses from hyperexcitability to hypoexcitability during the first weeks to months. The importance of evaluating elapsed time was recently emphasized in chronic epilepsy, were cortical excitability further reduced over years in poorly controlled patients, while it remained stable in moderately controlled patients (Pawley et al., 2017).

The potential of multimodal TMS to improve the diagnostic process depends not only on the ability to distinguish epilepsy patients from healthy controls, but even more on the ability to differentiate between first seizure patients diagnosed with and without epilepsy. Similar to the decrease in epilepsy patients, we found a non-significant trend towards decreased excitability in patients without epilepsy. These patients also showed increased $\mathrm{LICl}$ of the MEP amplitude and the N100 and P180 components for ISI $200 \mathrm{~ms}$, although not as prominent as in epilepsy patients. This indicates that, irrespective of the development of epilepsy, there seems to be a common underlying mechanism leading to changes in cortical excitability after a first seizure. 
For ISI 100 ms, we found facilitation in epilepsy patients instead of inhibition as in patients without epilepsy and healthy controls. Besides a common mechanism, there also appears to be a specific mechanism leading to excitability changes in epilepsy patients only. In the end, we would like to gain more insight into these epilepsyspecific changes, since this improves the diagnostic process and facilitates the decision whether or not to prescribe AEDs.

Due to the relatively small number of first seizure patients, we only performed analyses on a group level. However, ultimately the potential of TMS as a biomarker in epilepsy depends on the ability to differentiate at the level of an individual patient. Ideally, by combining the outcomes of the various TMS modalities, we end up with a single number indicating the likelihood of epilepsy. This could include feature selection methods combined with a Bayesian classifier, as applied in a recent study to determine the diagnostic accuracy on a group level (Kimiskidis et al., 2017). Based on our findings, single pulse TMS-EMG-EEG has probably no diagnostic value in epilepsy. In addition, we found some overlap in the paired pulse TMS-EMG and TMS-EEG outcomes. Feature selection methods can also be used to investigate whether a multimodal approach is actually necessary, or whether the use of a single TMS paradigm is sufficient.

\subsection{Conclusion}

Our findings indicate the potential of a multimodal TMS approach to improve the diagnostic process in epilepsy. At a group level, paired pulse TMS-EMG-EEG differentiated between healthy controls, patients with epilepsy and patients without epilepsy. Epilepsy patients showed significantly increased $\mathrm{LICl}$ of the N100 and P180 components for ISI $200 \mathrm{~ms}$ compared to healthy controls. Furthermore, epilepsy patients showed facilitation at ISI 100 ms instead of $\mathrm{LICl}$ as in patients without epilepsy. Therefore, potential diagnostic biomarkers include increased $\mathrm{LICI}$ at ISI $200 \mathrm{~ms}$ and facilitation at ISI $100 \mathrm{~ms}$. To establish the true potential of multimodal TMS, data of more patients is needed, which may enable relevant analysis at the individual patient level. 


\section{Acknowledgements}

The authors wish to thank Carin Eertman for her assistance during the TMS measurements and all the subjects for their participation.

\section{Funding}

This work was financed by the Dutch TWIN foundation for neuromodulation. The funding source played no role in the design of the study, collection, analysis and interpretation of the data, and writing of the manuscript. 



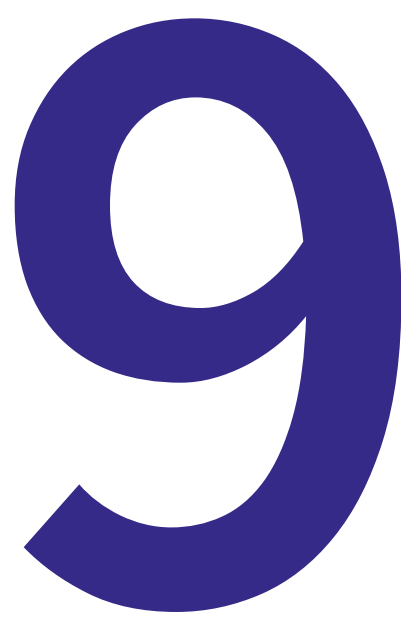

General discussion 
Epilepsy patients have an enduring predisposition to generate epileptic seizures (Fisher et al., 2005, 2014), due to an imbalance between excitation and inhibition resulting in an increased cortical excitability (McCormick and Contreras, 2001; McNamara, 1994). Even though 5\% of the population will experience an unprovoked seizure (Forsgren et al., 1996; Hauser et al., 1993; Sander and Shorvon, 1996), less than half of them develop epilepsy (Berg, 2008; Bouma et al., 2016). After a first seizure it is important to estimate the likelihood of more seizures, as the epilepsy diagnosis includes 1 ) at least two unprovoked seizures more than 24 hours apart, or 2) one unprovoked seizure with an increased risk of recurrence, or 3) a diagnosed epilepsy syndrome (Fisher et al., 2005, 2014). The tendency to generate seizures is reflected in the EEG by the presence of epileptiform discharges. However, the diagnostic yield of the EEG remains limited to 60-90\% (Rosenow et al., 2015), despite repeated and prolonged recordings which make the diagnostic process often time-consuming and labor intensive. This motivates the search for other biomarkers that could estimate the risk of seizure recurrence. We explored the potential of transcranial magnetic stimulation to assess the presumed abnormal cortical excitability in epilepsy.

In this thesis, we described our first steps towards the implementation of a multimodal TMS approach in epilepsy, with the aim to improve the diagnostic process. First, we evaluated the clinical feasibility of multimodal TMS in epilepsy. To assess the stability and robustness of TMS, we focused on the repeatability and positioning of the coil. Second, to explore potential sources of variation in assessment of cortical excitability, we studied if particular brain rhythms were correlated to changes in excitability. Third, we evaluated the clinical value of multimodal TMS in epilepsy. Besides our primary focus on the diagnostic value, we explored the added value for evaluating the therapeutic efficacy. Finally, we will provide suggestions for future research.

\subsection{Clinical feasibility of multimodal TMS}

The applicability of a multimodal TMS approach in epilepsy depends on both the added value to current diagnostics and clinical feasibility. TMS will most likely not be implemented in clinical practice, if it is only possible to detect significant differences in very specific conditions or by using a laborious measurement setup. The same applies to a simple TMS approach that shows no differences between epilepsy patients and healthy controls.

A multimodal TMS approach combines various TMS paradigms with promise to provide complementary information about cortical excitability. In this thesis, we used single and paired pulse TMS combined with EMG and EEG. As cortical excitability measures we evaluated the rMT, MEP amplitude, $\mathrm{LICl}$ and single and paired pulse TEP components. 
Regarding the clinical feasibility, we investigated whether these excitability measures could be assessed reliably at both a group and individual level. We tested the effect of time (repeatability), as well as minor changes in coil positioning.

\subsubsection{Repeatability}

In healthy subjects, we found a good repeatability for TMS-EMG and TMS-EEG on a group level. In Chapter 2, both rMT and LICI did not differ significantly between two sessions one week apart. This is in accordance with previously reported low intersession variability (Badawy et al., 2012) and high test-retest reliability for $\mathrm{LICl}$ (Farzan et al., 2010). We selected $\mathrm{LICl}$ as paired pulse TMS-EMG measure, since repeatability appears to be poorer and more variable for SICI and ICF (Badawy et al., 2012; Boroojerdi et al., 2000; Du et al., 2014; Dyke et al., 2018; Fleming et al., 2012; Hermsen et al., 2016; Maeda et al., 2002; Orth et al., 2003). In Chapter 4, we confirmed the high repeatability of the single (Casarotto et al., 2010; Kerwin et al., 2018; Lioumis et al., 2009) and paired pulse TEP over one week (Farzan et al., 2010; Premoli et al., 2014b). Recently, our group also found good reproducibility of the single pulse TEP during the day (ter Braack et al., 2018), while others showed good stability of the TEP throughout the TMS session (Casarotto et al., 2010; Kerwin et al., 2018). Only two studies evaluated long-term repeatability. They showed the stability of rMT across multiple TMS sessions over a period of six months (Dyke et al., 2018) or five years (Kimiskidis et al., 2004). Such longterm studies are also required for the other excitability measures to determine the true potential of TMS in longitudinal studies (e.g. to evaluate therapeutic efficacy).

Although repeatability was good at the group level, we found large variations in repeatability between individual subjects. Approximately half of the subjects described in Chapter 2 showed good LICI repeatability, while it was poor to moderate in the others. At least $\mathrm{LICI}$ repeatability seemed to be highly subject specific and showed a large inter-subject variation, just like other excitability measures (e.g. the LICl curve) (Du et al., 2014; Valls-Solé et al., 1992; Wassermann, 2002). Likewise, LICI reliability was poor when evaluating individualized facilitation-inhibition profiles three weeks apart (Du et al., 2014). Nevertheless, they indicate that individuals have unique profiles that remain relativity stable over time, despite large variances in profiles across subjects. Therefore, it might be better to evaluate specific characteristics of the facilitationinhibition profile, like the peak inhibition or the inhibition range. To understand what causes the inter-subject variability in repeatability, we recommend to perform studies with multiple repeated sessions and longer inter-session intervals. When subjects consistently show either good or poor repeatability over multiple sessions, it indeed points towards a subject specific component (e.g. individual sulcus anatomy). In this case, it is still possible to reliably measure changes over time in subjects 
with a good repeatability in order to evaluate therapeutic efficacy. However, when repeatability varies across sessions, it points towards differences in external factors (e.g. coil positioning or intake of neuroactive substances). In this case, it is possible that changes over time, due to a poor repeatability, are erroneously interpreted as treatment effects.

\subsubsection{Positioning of the coil}

In 1985, TMS was introduced as a non-invasive and safe technique that is fast and easy to use in a clinical environment (Barker et al., 1985). Today, manual positioning of the coil is still widely used and preferred, due to its speed and ease of use. However, neuronavigation systems are often recommended to ensure accurate coil positioning as well as stability during the session (Lefaucheur, 2010; Sparing et al., 2010). In Chapter 2, we found minimal differences in LICI repeatability for manual and robotguided coil positioning. Furthermore, in Chapter 4 we showed that the positioning method did not significantly affected the single and paired pulse TEP. Accordingly, no significant differences were found in $\mathrm{SICl}$ and ICF reliability between hand-held and navigated positioning (Fleming et al., 2012). Others reported no differences for the intra- and inter-subject variability of MEP amplitude (Gugino et al., 2001; Jung et al., 2010). Only one study showed that neuronavigation can significantly reduce the intra-subject variation, but it still remained very high (Julkunen et al., 2009). Overall, the added value of neuronavigation seems very limited, whereas a MRI scan is required and coil positioning takes about 1.5 hours (Gugino et al., 2001). If manual coil positioning appears to be sufficient during multimodal TMS, this would make the clinical applicability of TMS as a biomarker in epilepsy much easier.

Accurate positioning consists of two parts. First, the coil should be positioned precisely on the hotspot and second, the coil should be held in this position during the entire session. Regarding the first part, we showed in Chapter 3 that the largest MEP amplitudes were never evoked at the initially defined hotspot using function-guided navigation. This method determines the hotspot by visually inspecting the MEP amplitude while stimulating the motor area. During this procedure we usually applied less than ten pulses per location, while the trial-to-trial variation in MEP amplitude is known to be large (Goldsworthy et al., 2016; Wassermann, 2002). Recent studies suggest that at least ten pulses (Cavaleri et al., 2017) or even up to thirty pulses (Goldsworthy et al., 2016) are needed to reliably assess the amplitude. Thus, maybe increasing the number of pulses makes it possible to accurately determine the hotspot using functionguided navigation. Furthermore, instead of using the default angle of $45^{\circ}$ away from the midline, coil orientation could be adjusted. Determining the hotspot is also functionguided for neuronavigation, however, here the orientation is optimized so that the 
induced electrical currents flow perpendicular to the sulcus (Gugino et al., 2001; Raffin et al., 2015). When imaging data is missing, the coil may be rotated once the hotspot is determined, to find the orientation that results in the largest MEP amplitudes.

Regarding the second part, the effect of a change in coil positioning was evaluated in Chapter 3. On a group level, we found that the MEP amplitude, $\mathrm{LICl}$ and single pulse TEP were not significantly affected by a small change in either coil location or orientation. As long as these changes remain smaller than $10 \mathrm{~mm}$ and $20^{\circ}$, manual holding of the coil during the TMS session seems sufficiently accurate. In clinical practice, this should be feasible for experienced investigators. Only for relatively large changes in coil location $(>1 \mathrm{~cm})$ and orientation $\left(>45^{\circ}\right)$, others reported a significant decrease in MEP and TEP amplitudes (Casarotto et al., 2010; Julkunen et al., 2009; Jung et al., 2010; Komssi et al., 2002; Sparing et al., 2008). However, for individual subjects we showed that a 2 or 5 $\mathrm{mm}$ change in location and $10^{\circ}$ change in orientation can already result in significant differences in MEP amplitude, $\mathrm{LICl}$ and single pulse TEP. Our findings indicate that a high accuracy in coil positioning is especially required to reliably assess cortical excitability in individuals, but that it is of less importance when comparing groups. We consider the use of manual coil positioning in clinical practice to be sufficient, with the strong advice to search for ways to reduce the large intra- and inter-subject variability of excitability measures. Other methods, such as neuronavigation or robot-guided navigation, are not performing better, while they are less suitable for use in clinical practice.

\subsubsection{Stability and robustness of multimodal TMS}

Multimodal TMS appears to be very stable and robust when performing group analyses. We found good repeatability and showed that minor changes in coil position did not affect the various excitability measures. The majority of TMS studies report outcomes on a group level, either comparing patients to controls, or pre- to post-intervention sessions. Ultimately, however, we would like to make clinical decisions at the subject level. For this, individual patient data should either be compared to reference values for diagnostic purposes or to earlier measurements in the same patient for followup or treatment evaluation purposes. Unfortunately, we found that the stability and robustness of multimodal TMS were less when performing subject analyses. Individual subjects showed a large variability in repeatability and their response to minor changes in coil positioning.

The large intra- and inter-subject variability of excitability measures remains a major issue that may ultimately limit the clinical applicability of TMS (Ni and Chen, 2015; Wassermann, 2002). This variability can be partially reduced by increasing the number 
of pulses (Chang et al., 2016; Goldsworthy et al., 2016) and optimizing the coil positioning (Amassian et al., 1989a; Hess et al., 1987; Schmidt et al., 2015). However, despite these relatively simple methodological adjustments, the variability remains high. Furthermore, part of the inter-subject variability might be due to differences in sleep pattern (Huber et al., 2013) or the intake of neuroactive substances (e.g. caffeine, alcohol or nicotine) (Cerqueira et al., 2006; Kähkönen et al., 2003; Lang et al., 2008; Orth et al., 2005; Ziemann et al., 1995) between individuals. In this thesis, we have deliberately chosen not to compensate for these factors, because clinically relevant changes in cortical excitability should outweigh these potentially confounding effects. Moreover, imposing restrictions can make it more difficult to perform TMS shortly after the first seizure. Finally, part of these variations are caused by fluctuations in physiological processes which are more difficult to control, such as the state of ongoing brain oscillations (Bergmann et al., 2012; Sauseng et al., 2009), the hormonal balance (Hattemer et al., 2007; Smith et al., 1999; Zoghi et al., 2015), the level of muscle preactivation (Darling et al., 2006; Hess et al., 1987) and the attention level and arousal state of subjects (Conte et al., 2007; Mars et al., 2007; Silvanto and Pascual-Leone, 2008). Increasing our understanding of the underlying mechanisms of cortical excitability might enable a way to regulate these physiological processes. In particular, we focused on the state of ongoing brain oscillations.

\subsection{Variations in assessment of cortical excitability}

Biological fluctuations in cortical excitability are a candidate mechanism involved in the trial-to-trial variation in MEPs (Amassian et al., 1989b; Ferreri et al., 2014; Kiers et al., 1993). In Chapter 5, we found significant temporal clustering of TMS evoked muscle responses suggesting an underlying determinism of cortical excitability rather than random fluctuations. Approximately half of the subjects showed a significant correlation between the MEP amplitude and the phase of infraslow activity, defined as rhythms with frequencies below $0.1 \mathrm{~Hz}$. While this makes a contribution of infraslow activity as a modulator of cortical excitability likely, faster oscillations in the alpha, beta and gamma bands are probably involved as well (Berger et al., 2014; Iscan et al., 2016; Mäki and Ilmoniemi, 2010a; Sauseng et al., 2009; Schulz et al., 2014; Zarkowski et al., 2006; Zrenner et al., 2018). To confirm a causal relation between cortical excitability and brain oscillations, more research is needed. Preferably these studies include activity in the infraslow band by using full-band EEG data.

A candidate technique to reduce response variation is to apply TMS pulses during a specific phase of ongoing oscillations, in our case the infraslow activity. The combination of TMS with EEG enables such a brain-state triggered TMS approach 
(Zrenner et al., 2016). A recent study used a closed-loop system to apply TMS pulses as a function of the simultaneously recorded EEG (Zrenner et al., 2018). Instead of infraslow activity, TMS was synchronized to the phase of ongoing mu-oscillations recorded from the sensorimotor cortex. Significantly higher MEP amplitudes were found for stimulation during the negative peak of the mu-oscillation (high-excitability state), than during the positive peak (low-excitability state) (Zrenner et al., 2018).

A next step would be to evaluate whether brain-state triggered TMS can reduce the variability of other excitability measures as well. A decrease of the intra- and inter-subject variability increases the ability to detect significant differences between conditions (e.g. epilepsy patients and healthy controls).

\subsection{Clinical value of multimodal TMS in epilepsy}

Hyperexcitable cortical networks are thought to be associated with a tendency to generate seizures in epilepsy patients (McCormick and Contreras, 2001; McNamara, 1994). The systematic overview in Chapter 6 reported that this is indeed shown by various studies on cortical excitability in drug naïve epilepsy patients. Since the use of AEDs can significantly affect cortical excitability (Ziemann et al., 2015), we focused on drug naïve patients. Thus, we only analyzed TMS-EMG data, where findings were most consistent for the paired pulse paradigm. Instead of inhibition, facilitation was found at ISIs 2, 5, 250 and $300 \mathrm{~ms}$ in drug naïve patients. However, to establish the true potential of TMS as a diagnostic biomarker in epilepsy we recommended 1) to reproduce these promising paired pulse TMS-EMG results and 2) to combine TMS with EEG in an attempt to move towards the level of an individual patient.

\subsubsection{Improving the diagnostic process}

In this thesis, we applied multimodal TMS to assess cortical excitability in healthy controls and drug naïve first seizure patients, including those eventually diagnosed with epilepsy. In healthy controls we found, as expected, $\mathrm{LICl}$ of the EMG response for ISIs between 100-300 ms. As shown in Chapter 4, $\mathrm{LICl}$ of the EEG response was characterized by strong inhibition of the late TEP components. Whereas the early P30, N45 and P60 components of the paired pulse TEP were similar to those of the single pulse TEP, the late $\mathrm{N} 100$ and P180 components were significantly attenuated. In contrast, epilepsy patients showed significantly increased $\mathrm{LICl}$ of the $\mathrm{N} 100$ and P180 components at ISI $200 \mathrm{~ms}$. In Chapter 8, we additionally found facilitation of the MEP amplitude at ISI $100 \mathrm{~ms}$ in epilepsy patients and LICI in patients without epilepsy. These findings indicate the potential of multimodal TMS to improve the diagnostic process in epilepsy. On a group level, paired pulse TMS-EMG-EEG could differentiate patients 
diagnosed with epilepsy from those without epilepsy and healthy controls. For single pulse TMS, we found no significant differences between the three subject categories.

Potential diagnostic biomarkers for epilepsy include increased $\mathrm{LICl}$ (TMS-EMG) and $\mathrm{LICl}$ of the N100 and P180 components (TMS-EEG) at ISI $200 \mathrm{~ms}$, as well as facilitation (TMS-EMG) at ISI $100 \mathrm{~ms}$. These outcomes are in contrast to the previously reported facilitation (TMS-EMG) at ISI 250 and 300 ms, as described in Chapter 6. The fact that we could not replicate the promising results found by Badawy and colleagues can have several reasons. First, they frequently reused data of patients and controls, making their studies less independent (Badawy et al., 2017). Second, their data analysis and representation showed inconsistencies and numerous errors (Badawy et al., 2017; Bauer et al., 2017). Finally, there were methodological differences between our study and theirs, such as using a figure-of-eight versus a circular coil, applying 50 versus 10 repetitions, waiting $\pm 4 \mathrm{~s}$ versus $15 \mathrm{~s}$ between pairs of paired pulses and measuring patients around 1 month versus 1-2 weeks after their first seizure. In particular, the difference in time between the first seizure and the TMS session could explain our finding of a decrease rather than an increase in cortical excitability in epilepsy patients. When drug naïve patients were measured months to years after their initial seizure, hyperactivation of inhibitory neuronal circuits was observed, which is thought to act as a protective mechanism to prevent seizure recurrence (Lee et al., 2015; Silbert et al., 2015). Similarly, hyperexcitability was found for 24 hours prior to a seizure (Badawy et al., 2008), while excitability was significantly decreased for 24-48 hours afterwards (Badawy et al., 2008; Delvaux et al., 2001).

Despite the potential of multimodal TMS to improve the diagnostic process in epilepsy, data of more first seizure patients is needed to enable analysis at the level of an individual patient. Including more epilepsy patients makes it also possible to analyze data of generalized and focal epilepsy patients separately. Finding consistency between both types of epilepsy increases the potential of TMS as a diagnostic biomarker. So far, we showed the clinical feasibility of a multimodal TMS approach. In addition, we found potential diagnostic biomarkers indicating the clinical value of TMS in epilepsy. These are all important steps towards the implementation of a multimodal TMS approach in epilepsy to improve the diagnostic process.

\subsubsection{Evaluating therapeutic efficacy}

Since treatment success depends on achieving seizure freedom, a biomarker that estimates the risk of seizure recurrence might also shorten the time needed to assess the treatment response. Currently, we explore the potential of multimodal TMS to evaluate the therapeutic efficacy of AEDs (trial ID: NL49901.044.14). Diagnosed 
epilepsy patients are measured before and after (8-12 weeks and 1 year) starting AED treatment. Here, the potential to become a biomarker depends on the ability to differentiate between responders (well-controlled) and non-responders (poorcontrolled). Therefore, excitability measures suitable for treatment evaluation may differ from those found for the diagnostic process.

Eventually, approximately $30 \%$ of the epilepsy patients will continue to have seizures, despite the use of AEDs (Brodie et al., 2012). In Chapter 7, we evaluated differences between these refractory epilepsy patients and healthy controls using paired pulse TMS-EMG data measured in four centers. Initially, no significant differences in $\mathrm{LICl}$ were found. Due to methodological differences between one center and the others, the same analyses were performed without the data from Center 2 . This time, we found significant differences in $\mathrm{LICl}$ for ISIs 100 and 200 ms between healthy controls and refractory focal epilepsy patients. In Chapter 8 , we found differences between controls and epilepsy patients for these same ISIs. Still, no differences were found between refractory generalized epilepsy patients and controls, nor between generalized and focal patients. Based on these findings, paired pulse TMS-EMG in epilepsy has probably no diagnostic value. However, including EEG data might improve the ability to differentiate between healthy controls and refractory epilepsy patients.

\subsection{Future perspectives}

Pharmaco-TMS studies can help to find potential biomarkers to evaluate therapeutic efficacy. At the Centre for Human Drug Research (CHDR) in Leiden we are currently evaluating the effect of two commonly used AEDs on the cortical excitability in healthy controls (trial ID: NL62207.056.17). Next, we would like to perform TMS-EMG-EEG, combined with pharmacokinetics and -dynamics, in drug naïve epilepsy patients prior to their first AED dose up to 24 hours post-dose. Pharmaco-TMS offers the possibility to test existing and novel drugs in a highly controlled manner, as well as to quantify the confounding effects of AEDs on cortical excitability. In addition, it can increase our understanding of the underlying mechanisms by studying direct relations between excitability measures and neurotransmission pathways, using drugs which specific modes of action (Ziemann, 2017). So far, single pulse pharmaco-TMS-EEG demonstrated that the N45 and N100 components are related to GABA-A and GABA-B receptor mediated inhibition, respectively (Premoli et al., 2014a). While, paired pulse pharmaco-TMS-EEG showed largely identical TEP modulation for SICI (ISI $2 \mathrm{~ms}$ ) and LICI (ISI 100 ms), including highly similar pharmacological profiles (Premoli et al., 2018). 
Combining TMS and EEG makes the data analysis and interpretation challenging, because the TEP is contaminated with artifacts (Ilmoniemi et al., 2015; Ilmoniemi and Kičić, 2010). Besides activating scalp muscles, TMS induces a somatosensory evoked potential (SSEP) and auditory evoked potential (AEP) (Ilmoniemi et al., 2015). To make the applicability of TMS-EEG easier, we strongly encourage recent initiatives for the development of a (fully) automated artifact rejection algorithm (Atluri et al., 2016; Rogasch et al., 2017; Wu et al., 2018). Ideally, this algorithm is tested on both single and paired pulse TMS-EEG data containing the different types of artifacts to varying degrees. Simultaneously, it is possible to evaluate which correction methods are needed for interpretation of the paired pulse TEP, which is not straightforward as the conditioning TEP is still ongoing when applying the test pulse (Daskalakis et al., 2008; Premoli et al., 2014b). Even though we are aware that the TEP contains SSEP and AEP components, we are unaware of the extent of this contribution. A recent study applied sham stimulation to the parietal and frontal cortex, mimicking the somatosensory and auditory sensations evoked by real TMS. They showed substantial similarities between TMS and sham evoked EEG responses (Conde et al., 2019). Because of the possible implications, it is important that these findings are reproduced, including assessment of these potential contributions when stimulating motor areas.

\subsection{Conclusion}

In this thesis we described our first steps towards the implementation of a multimodal TMS approach in epilepsy. We showed the clinical feasibility and potential of multimodal TMS to improve the diagnostic process. On a group level, we found a good repeatability and no significant effect of minor changes in coil positioning for the various excitability measures in healthy subjects. However, the stability and robustness of multimodal TMS were less on a subject level, due to a large variability across individuals. We showed that cortical excitability is correlated with infraslow activity. Thus, one way to reduce the intra- and inter-subject variability might be to target stimulation to a specific phase of the infraslow activity or other ongoing oscillations. Furthermore, we could differentiate first seizure patients diagnosed with epilepsy from those without epilepsy and healthy controls. Potential biomarkers to improve the diagnostic process include increased LICI at ISI $200 \mathrm{~ms}$ and facilitation at ISI $100 \mathrm{~ms}$. 




\section{References}

Abbruzzese, G. and Trompetto, C. (2002). Clinical and research methods for evaluating cortical excitability. Journal of Clinical Neurophysiology, 19(4):307-321.

Aladjalova, N. A. (1957). Infra-slow rhythmic oscillations of the steady potential of the cerebral cortex. Nature, 179(4567):957-959.

Aladjalova, N. A. (1964). Slow electrical processes in the brain. Elsevier, Amsterdam. OCLC: 879035020.

Albrecht, D. and Gabriel, S. (1994). Very slow oscillations of activity in geniculate neurones of urethaneanaesthetized rats. Neuroreport, 5(15):1909-1912.

Albrecht, D., Royl, G., and Kaneoke, Y. (1998). Very slow oscillatory activities in lateral geniculate neurons of freely moving and anesthetized rats. Neuroscience Research, 32(3):209-220.

Amassian, V. E., Cracco, R. Q., and Maccabee, P. J. (1989a). Focal stimulation of human cerebral cortex with the magnetic coil: A comparison with electrical stimulation. Electroencephalography and Clinical Neurophysiology/Evoked Potentials Section, 74(6):401-416.

Amassian, V. E., Cracco, R. Q., Maccabee, P. J., Cracco, J. B., Rudell, A., and Eberle, L. (1989b). Suppression of visual perception by magnetic coil stimulation of human occipital cortex. Electroencephalography and Clinical Neurophysiology/Evoked Potentials Section, 74(6):458-462.

Amzica, F. and Steriade, M. (2000). Neuronal and glial membrane potentials during sleep and paroxysmal oscillations in the neocortex. The Journal of Neuroscience: The Official Journal of the Society for Neuroscience, 20(17):6648-6665.

ANT Neuro (accessed on 19 March 2018). ANT Smartmove datasheet. https://www.ant-neuro.com /sites/default/files/120913_ANT_robotarm_datasheet-2.pdf.

Atluri, S., Frehlich, M., Mei, Y., Garcia Dominguez, L., Rogasch, N. C., Wong, W., et al. (2016). TMSEEG: A MATLAB-based graphical user interface for processing electrophysiological signals during transcranial magnetic stimulation. Frontiers in Neural Circuits, 10.

Awiszus, F. (2011). Fast estimation of transcranial magnetic stimulation motor threshold: Is it safe? Brain Stimulation, 4(1):58-59.

Awiszus, F. (2012). On relative frequency estimation of transcranial magnetic stimulation motor threshold. Clinical Neurophysiology, 123(11):2319-2320.

Badawy, R. A. B., Curatolo, J. M., Newton, M., Berkovic, S. F., and Macdonell, R. A. L. (2006). Sleep deprivation increases cortical excitability in epilepsy - Syndrome-specific effects. Neurology, 67(6):1018-1022.

Badawy, R. A. B., Curatolo, J. M., Newton, M., Berkovic, S. F., and Macdonell, R. A. L. (2007). Changes in cortical excitability differentiate generalized and focal epilepsy. Annals of Neurology, 61(4):324-331.

Badawy, R. A. B. and Jackson, G. D. (2012). Cortical excitability in migraine and epilepsy: A common feature? Journal of Clinical Neurophysiology, 29(3):244-249.

Badawy, R. A. B., Jackson, G. D., Berkovic, S. F., and Macdonell, R. A. L. (2012). Inter-session repeatability of cortical excitability measurements in patients with epilepsy. Epilepsy Research, 98(2-3):182-186.

Badawy, R. A. B., Jackson, G. D., Berkovic, S. F., and Macdonell, R. A. L. (2013a). Cortical excitability and refractory epilepsy: A three-year longitudinal transcranial magnetic stimulation study. International Journal of Neural Systems, 23(01):1250030. 
Badawy, R. A. B., Macdonell, R. A. L., Berkovic, S. F., Newton, M. R., and Jackson, G. D. (2010a). Predicting seizure control: Cortical excitability and antiepileptic medication. Annals of Neurology, 67(1):64-73.

Badawy, R. A. B., Macdonell, R. A. L., Berkovic, S. F., Vogrin, S. J., Jackson, G. D., and Cook, M. J. (2017). Reply: Transcranial magnetic stimulation as a biomarker for epilepsy. Brain, 140(3):e19-e19.

Badawy, R. A. B., Macdonell, R. A. L., Jackson, G. D., and Berkovic, S. F. (2008). The peri-ictal state: Cortical excitability changes within $24 \mathrm{~h}$ of a seizure. Brain, 132(4):1013-1021.

Badawy, R. A. B., Macdonell, R. A. L., Jackson, G. D., and Berkovic, S. F. (2009). Why do seizures in generalized epilepsy often occur in the morning? Neurology, 73(3):218-222.

Badawy, R. A. B., Macdonell, R. A. L., Jackson, G. D., and Berkovic, S. F. (2010b). Can changes in cortical excitability distinguish progressive from juvenile myoclonic epilepsy? Epilepsia, 51(10):2084-2088.

Badawy, R. A. B., Strigaro, G., and Cantello, R. (2014a). TMS, cortical excitability and epilepsy: The clinical impact. Epilepsy Research, 108(2):153-161.

Badawy, R. A. B., Tarletti, R., Mula, M., Varrasi, C., and Cantello, R. (2011). The routine circular coil is reliable in paired-TMS studies. Clinical Neurophysiology, 122(4):784-788.

Badawy, R. A. B., Vogrin, S. J., Lai, A., and Cook, M. J. (2013b). Capturing the epileptic trait: Cortical excitability measures in patients and their unaffected siblings. Brain, 136(4):1177-1191.

Badawy, R. A. B., Vogrin, S. J., Lai, A., and Cook, M. J. (2013c). Cortical excitability changes correlate with fluctuations in glucose levels in patients with epilepsy. Epilepsy \& Behavior, 27(3):455-460.

Badawy, R. A. B., Vogrin, S. J., Lai, A., and Cook, M. J. (2013d). Patterns of cortical hyperexcitability in adolescent/adult-onset generalized epilepsies. Epilepsia, 54(5):871-878.

Badawy, R. A. B., Vogrin, S. J., Lai, A., and Cook, M. J. (2013e). The cortical excitability profile of temporal lobe epilepsy. Epilepsia, 54(11):1942-1949.

Badawy, R. A. B., Vogrin, S. J., Lai, A., and Cook, M. J. (2014b). On the midway to epilepsy: Is cortical excitability normal in patients with isolated seizures? International Journal of Neural Systems, 24(02):1430002.

Badawy, R. A. B., Vogrin, S. J., Lai, A., and Cook, M. J. (2015). Does the region of epileptogenicity influence the pattern of change in cortical excitability? Clinical Neurophysiology, 126(2):249-256.

Balslev, D., Braet, W., McAllister, C., and Miall, R. C. (2007). Inter-individual variability in optimal current direction for transcranial magnetic stimulation of the motor cortex. Journal of Neuroscience Methods, 162(1-2):309-313.

Barker, A. T., Jalinous, R., and Freeston, I. L. (1985). Non-invasive magnetic stimulation of human motor cortex. The Lancet, 325(8437):1106-1107.

Bates, D., Mächler, M., Bolker, B., and Walker, S. (2015). Fitting linear mixed-effects models using Ime4. Journal of Statistical Software, 67(1).

Bauer, P. R., de Goede, A. A., Stern, W. M., Pawley, A. D., Chowdhury, F. A., Helling, R. M., et al. (2018). Long-interval intracortical inhibition as biomarker for epilepsy: a transcranial magnetic stimulation study. Brain, 141(2):409-421.

Bauer, P. R., de Goede, A. A., ter Braack, E. M., van Putten, M. J. A. M., Gill, R. D., and Sander, J. W. (2017). Transcranial magnetic stimulation as a biomarker for epilepsy. Brain, 140(3):e18-e18.

Bauer, P. R., Kalitzin, S., Zijlmans, M., Sander, J. W., and Visser, G. H. (2014). Cortical excitability as a potential clinical marker of epilepsy: A review of the clinical application of transcranial magnetic stimulation. International Journal of Neural Systems, 24(02):1430001.

Berg, A. T. (2008). Risk of recurrence after a first unprovoked seizure: Risk of Recurrence. Epilepsia, 49:13-18.

Berg, A. T., Berkovic, S. F., Brodie, M. J., Buchhalter, J., Cross, J. H., van Emde Boas, W., et al. (2010). Revised terminology and concepts for organization of seizures and epilepsies: Report of the ILAE Commission on Classification and Terminology, 2005-2009. Epilepsia, 51(4):676-685. 
Berger, B., Minarik, T., Liuzzi, G., Hummel, F. C., and Sauseng, P. (2014). EEG oscillatory phase-dependent markers of corticospinal excitability in the resting brain. BioMed Research International, 2014:1-8.

Bergmann, T. O., Molle, M., Schmidt, M. A., Lindner, C., Marshall, L., Born, J., et al. (2012). EEG-guided transcranial magnetic stimulation reveals rapid shifts in motor cortical excitability during the human sleep slow oscillation. Journal of Neuroscience, 32(1):243-253.

Bolden, L. B., Griffis, J. C., Pati, S., and Szaflarski, J. P. (2017). Cortical excitability and neuropsychological functioning in healthy adults. Neuropsychologia, 102:190-196.

Bonato, C., Miniussi, C., and Rossini, P. M. (2006). Transcranial magnetic stimulation and cortical evoked potentials: A TMS/EEG co-registration study. Clinical Neurophysiology, 117(8):1699-1707.

Boon, P., De Cock, E., Mertens, A., and Trinka, E. (2018). Neurostimulation for drug-resistant epilepsy: a systematic review of clinical evidence for efficacy, safety, contraindications and predictors for response. Current Opinion in Neurology, page 1.

Boroojerdi, B., Kopylev, L., Battaglia, F., Facchini, S., Ziemann, U., Muellbacher, W., et al. (2000). Reproducibility of intracortical inhibition and facilitation using the paired-pulse paradigm. Muscle \& Nerve, 23(10):1594-1597.

Bouma, H. K., Labos, C., Gore, G. C., Wolfson, C., and Keezer, M. R. (2016). The diagnostic accuracy of routine electroencephalography after a first unprovoked seizure. European Journal of Neurology, 23(3):455-463.

Bragin, A., Engel, J., Wilson, C. L., Fried, I., and Buzsáki, G. (1999). High-frequency oscillations in human brain. Hippocampus, 9(2):137-142.

Brigo, F., Bongiovanni, L. G., Nardone, R., Trinka, E., Tezzon, F., Fiaschi, A., et al. (2013). Visual cortex hyperexcitability in idiopathic generalized epilepsies with photosensitivity: A TMS pilot study. Epilepsy \& Behavior, 27(2):301-306.

Brigo, F., Storti, M., Benedetti, M. D., Rossini, F., Nardone, R., Tezzon, F., et al. (2012). Resting motor threshold in idiopathic generalized epilepsies: a systematic review with meta-analysis. Epilepsy Research, 101(12):3-13.

Brodie, M. J., Barry, S. J. E., Bamagous, G. A., Norrie, J. D., and Kwan, P. (2012). Patterns of treatment response in newly diagnosed epilepsy. Neurology, pages WNL-0b013e3182563b19.

Brodtmann, A., Macdonell, R. A. L., Gilligan, A. K., Curatolo, J., and Berkovic, S. F. (1999). Cortical excitability and recovery curve analysis in generalized epilepsy. Neurology, 53(6):1347-1347.

Bunse, T., Wobrock, T., Strube, W., Padberg, F., Palm, U., Falkai, P., et al. (2014). Motor cortical excitability assessed by transcranial magnetic stimulation in psychiatric disorders: A systematic review. Brain Stimulation, 7(2):158-169.

Buzsáki, G. and Draguhn, A. (2004). Neuronal oscillations in cortical networks. Science (New York, N.Y.), 304(5679):1926-1929.

Buzsáki, G. and Watson, B. O. (2012). Brain rhythms and neural syntax: implications for efficient coding of cognitive content and neuropsychiatric disease. Dialogues in Clinical Neuroscience, 14(4):345-367.

Calancie, B., Nordin, M., Wallin, U., and Hagbarth, K. E. (1987). Motor-unit responses in human wrist flexor and extensor muscles to transcranial cortical stimuli. Journal of Neurophysiology, 58(5):1168-1185.

Callaghan, B., Schlesinger, M., Rodemer, W., Pollard, J., Hesdorffer, D., Allen Hauser, W., et al. (2011). Remission and relapse in a drug-resistant epilepsy population followed prospectively: Drug-Resistant Epilepsy Population. Epilepsia, 52(3):619-626.

Cantello, R., Civardi, C., Cavalli, A., Varrasi, C., Tarletti, R., Monaco, F., et al. (2000). Cortical excitability in cryptogenic localization-related epilepsy: Interictal transcranial magnetic stimulation studies. Epilepsia, 41(6):694-704.

Cantello, R., Civardi, C., Varrasi, C., Vicentini, R., Cecchin, M., Boccagni, C., et al. (2006). Excitability of the human epileptic cortex after chronic valproate: A reappraisal. Brain Research, 1099(1):160-166. 
Casarotto, S., Romero Lauro, L. J., Bellina, V., Casali, A. G., Rosanova, M., Pigorini, A., et al. (2010). EEG responses to TMS are sensitive to changes in the perturbation parameters and repeatable over time. PLoS ONE, 5(4):e10281.

Cash, R. F. H., Ziemann, U., Murray, K., and Thickbroom, G. W. (2010). Late cortical disinhibition in human motor cortex: A triple-pulse transcranial magnetic stimulation study. Journal of Neurophysiology, 103(1):511-518.

Caux-Dedeystère, A., Derambure, P., and Devanne, H. (2015). Late cortical disinhibition in relaxed versus active hand muscles. Neuroscience, 298:52-62.

Cavaleri, R., Schabrun, S. M., and Chipchase, L. S. (2015). Determining the number of stimuli required to reliably assess corticomotor excitability and primary motor cortical representations using transcranial magnetic stimulation (TMS): A protocol for a systematic review and meta-analysis. Systematic Reviews, 4(1).

Cavaleri, R., Schabrun, S. M., and Chipchase, L. S. (2017). The number of stimuli required to reliably assess corticomotor excitability and primary motor cortical representations using transcranial magnetic stimulation (TMS): a systematic review and meta-analysis. Systematic Reviews, 6(1).

Cerqueira, V., de Mendonça, A., Minez, A., Dias, A., and de Carvalho, M. (2006). Does caffeine modify corticomotor excitability? Neurophysiologie Clinique/Clinical Neurophysiology, 36(4):219-226.

Chang, W. H., Fried, P. J., Saxena, S., Jannati, A., Gomes-Osman, J., Kim, Y.-H., et al. (2016). Optimal number of pulses as outcome measures of neuronavigated transcranial magnetic stimulation. Clinical Neurophysiology.

Chen, R., Classen, J., Gerloff, C., Celnik, P., Wassermann, E. M., Hallett, M., et al. (1997). Depression of motor cortex excitability by low-frequency transcranial magnetic stimulation. Neurology, 48(5):1398-1403.

Chen, R., Cros, D., Curra, A., Di Lazzaro, V., Lefaucheur, J.-P., Magistris, M. R., et al. (2008). The clinical diagnostic utility of transcranial magnetic stimulation: Report of an IFCN committee. Clinical Neurophysiology, 119(3):504-532.

Chipchase, L., Schabrun, S., Cohen, L., Hodges, P., Ridding, M., Rothwell, J., et al. (2012). A checklist for assessing the methodological quality of studies using transcranial magnetic stimulation to study the motor system: An international consensus study. Clinical Neurophysiology, 123(9):1698-1704.

Chowdhury, F. A., Pawley, A. D., Ceronie, B., Nashef, L., Elwes, R. D. C., and Richardson, M. P. (2015). Motor evoked potential polyphasia - A novel endophenotype of idiopathic generalized epilepsy. Neurology, 84(13):1301-1307.

Cincotta, M., Giovannelli, F., Borgheresi, A., Balestrieri, F., Toscani, L., Zaccara, G., et al. (2010). Optically tracked neuronavigation increases the stability of hand-held focal coil positioning: Evidence from "transcranial" magnetic stimulation-induced electrical field measurements. Brain Stimulation, 3(2):119123.

Cincotta, M., Giovannelli, F., Borgheresi, A., Tramacere, L., Viggiano, M. P., and Zaccara, G. (2015). A metaanalysis of the cortical silent period in epilepsies. Brain Stimulation, 8(4):693-701.

Cohen, J. (1988). Statistical power analysis for the behavioral sciences. L. Erlbaum Associates, Hillsdale, N.J, 2nd ed edition.

Cohen, L. G., Roth, B. J., Nilsson, J., Dang, N., Panizza, M., Bandinelli, S., et al. (1990). Effects of coil design on delivery of focal magnetic stimulation. Technical considerations. Electroencephalography and Clinical Neurophysiology, 75(4):350-357.

Conde, V., Tomasevic, L., Akopian, I., Stanek, K., Saturnino, G. B., Thielscher, A., et al. (2019). The nontranscranial TMS-evoked potential is an inherent source of ambiguity in TMS-EEG studies. Neurolmage, 185:300-312.

Conte, A., Gilio, F., lezzi, E., Frasca, V., Inghilleri, M., and Berardelli, A. (2007). Attention influences the excitability of cortical motor areas in healthy humans. Experimental Brain Research, 182(1):109-117. 
Darling, W. G., Wolf, S. L., and Butler, A. J. (2006). Variability of motor potentials evoked by transcranial magnetic stimulation depends on muscle activation. Experimental Brain Research, 174(2):376-385.

Daskalakis, Z. J., Farzan, F., Barr, M. S., Maller, J. J., Chen, R., and Fitzgerald, P. B. (2008). Long-interval cortical inhibition from the dorsolateral prefrontal cortex: a TMS-EEG study. Neuropsychopharmacology, 33(12):2860.

Daskalakis, Z. J., Molnar, G. F., Christensen, B. K., Sailer, A., Fitzgerald, P. B., and Chen, R. (2003). An automated method to determine the transcranial magnetic stimulation-induced contralateral silent period. Clinical Neurophysiology, 114(5):938-944.

Day, B. L., Thompson, P. D., Dick, J. P., Nakashima, K., and Marsden, C. D. (1987). Different sites of action of electrical and magnetic stimulation of the human brain. Neuroscience letters, 75(1):101-106.

de Goede, A. A., ter Braack, E. M., and van Putten, M. J. A. M. (2016). Single and paired pulse transcranial magnetic stimulation in drug naïve epilepsy. Clinical Neurophysiology, 127(9):3140-3155.

de Goede, A. A., ter Braack, E. M., and van Putten, M. J. A. M. (2018). Accurate coil positioning is important for single and paired pulse tms on the subject level. Brain Topography.

de Goede, A. A. and van Putten, M. J. A. M. (2017). Repeatability of long intracortical inhibition in healthy subjects. Clinical Neurophysiology Practice, 2:26-34.

Del Felice, A., Fiaschi, A., Bongiovanni, G. L., Savazzi, S., and Manganotti, P. (2011). The sleep-deprived brain in normals and patients with juvenile myoclonic epilepsy: A perturbational approach to measuring cortical reactivity. Epilepsy Research, 96(1-2):123-131.

Delvaux, V., Alagona, G., Gérard, P., De Pasqua, V., Delwaide, P. J., and de Noordhout, A. M. (2001). Reduced excitability of the motor cortex in untreated patients with de novo idiopathic "grand mal" seizures. Journal of Neurology, Neurosurgery \& Psychiatry, 71(6):772-776.

Deng, Z.-D., Lisanby, S. H., and Peterchev, A. V. (2013). Electric field depth-focality tradeoff in transcranial magnetic stimulation: Simulation comparison of 50 coil designs. Brain Stimulation, 6(1):1-13.

Deng, Z.-D., Peterchev, A. V., and Lisanby, S. H. (2008). Coil design considerations for deep-brain transcranial magnetic stimulation (dTMS). In Engineering in Medicine and Biology Society, 2008. EMBS 2008. 30th Annual International Conference of the IEEE, pages 5675-5679. IEEE.

Devanne, H., Lavoie, B. A., and Capaday, C. (1997). Input-output properties and gain changes in the human corticospinal pathway. Experimental Brain Research, 114(2):329-338.

Di Lazzaro, V., Oliviero, A., Mazzone, P., Insola, A., Pilato, F., Saturno, E., et al. (2001). Comparison of descending volleys evoked by monophasic and biphasic magnetic stimulation of the motor cortex in conscious humans. Experimental Brain Research, 141(1):121-127.

Di Lazzaro, V., Oliviero, A., Profice, P., Saturno, E., Pilato, F., Insola, A., et al. (1998a). Comparison of descending volleys evoked by transcranial magnetic and electric stimulation in conscious humans. Electroencephalography and Clinical Neurophysiology/Electromyography and Motor Control, 109(5):397401.

Di Lazzaro, V., Pilato, F., Dileone, M., Profice, P., Ranieri, F., Ricci, V., et al. (2007). Segregating two inhibitory circuits in human motor cortex at the level of GABAA receptor subtypes: A TMS study. Clinical Neurophysiology, 118(10):2207-2214.

Di Lazzaro, V., Restuccia, D., Oliviero, A., Profice, P., Ferrara, L., Insola, A., et al. (1998b). Magnetic transcranial stimulation at intensities below active motor threshold activates intracortical inhibitory circuits. Experimental Brain Research, 119(2):265-268.

Du, X., Summerfelt, A., Chiappelli, J., Holcomb, H. H., and Hong, L. E. (2014). Individualized brain inhibition and excitation profile in response to paired-pulse TMS. Journal of Motor Behavior, 46(1):39-48.

Dyke, K., Kim, S., Jackson, G. M., and Jackson, S. R. (2018). Reliability of single and paired pulse transcranial magnetic stimulation parameters across eight testing sessions. Brain Stimulation, 11(6):1393-1394. 
Elger, C. E. and Schmidt, D. (2008). Modern management of epilepsy: A practical approach. Epilepsy \& Behavior, 12(4):501-539.

Engel, J. J. (2008). Progress in epilepsy: reducing the treatment gap and the promise of biomarkers. Current opinion in neurology, 21(2):150-154.

Ertas, N. K., Gul, G., Altunhalka, A., and Kirbas, D. (2000). Cortical silent period following transcranial magnetic stimulation in epileptic patients. Epileptic Disorders, 2(3):137-140.

Farrance, I. and Frenkel, R. (2012). Uncertainty of measurement: a review of the rules for calculating uncertainty components through functional relationships. The Clinical Biochemist Reviews, 33(2):49-75.

Farzan, F., Barr, M. S., Levinson, A. J., Chen, R., Wong, W., Fitzgerald, P. B., et al. (2010). Reliability of longinterval cortical inhibition in healthy human subjects: A TMS-EEG study. Journal of Neurophysiology, 104(3):1339-1346.

Fecchio, M., Pigorini, A., Comanducci, A., Sarasso, S., Casarotto, S., Premoli, I., et al. (2017). The spectral features of EEG responses to transcranial magnetic stimulation of the primary motor cortex depend on the amplitude of the motor evoked potentials. PLOS ONE, 12(9):e0184910.

Ferrarelli, F., Massimini, M., Peterson, M. J., Riedner, B. A., Lazar, M., Murphy, M. J., et al. (2008). Reduced evoked gamma oscillations in the frontal cortex in schizophrenia patients: a TMS/EEG study. American Journal of Psychiatry, 165(8):996-1005.

Ferreri, F., Pasqualetti, P., Määttä, S., Ponzo, D., Ferrarelli, F., Tononi, G., et al. (2011). Human brain connectivity during single and paired pulse transcranial magnetic stimulation. Neurolmage, 54(1):90102.

Ferreri, F. and Rossini, P. M. (2013). TMS and TMS-EEG techniques in the study of the excitability, connectivity, and plasticity of the human motor cortex. Reviews in the Neurosciences, 24(4).

Ferreri, F., Vecchio, F., Ponzo, D., Pasqualetti, P., and Rossini, P. M. (2014). Time-varying coupling of EEG oscillations predicts excitability fluctuations in the primary motor cortex as reflected by motor evoked potentials amplitude: An EEG-TMS study: EEG Coupling and Cortical Excitability. Human Brain Mapping, 35(5):1969-1980.

Fisher, R. S., Acevedo, C., Arzimanoglou, A., Bogacz, A., Cross, J. H., Elger, C. E., et al. (2014). ILAE Official Report: A practical clinical definition of epilepsy. Epilepsia, 55(4):475-482.

Fisher, R. S., Cross, J. H., French, J. A., Higurashi, N., Hirsch, E., Jansen, F. E., et al. (2017). Operational classification of seizure types by the International League Against Epilepsy: Position paper of the ILAE Commission for classification and terminology. Epilepsia, 58(4):522-530.

Fisher, R. S., van Emde Boas, W., Blume, W., Elger, C., Genton, P., Lee, P., et al. (2005). Epileptic seizures and epilepsy: definitions proposed by the International League Against Epilepsy (ILAE) and the International Bureau for Epilepsy (IBE). Epilepsia, 46(4):470-472.

Fitzgerald, P. B., Daskalakis, Z. J., Hoy, K., Farzan, F., Upton, D. J., Cooper, N. R., et al. (2008). Cortical inhibition in motor and non-motor regions: a combined TMS-EEG study. Clinical EEG and neuroscience, 39(3):112117.

Fitzgerald, P. B., Maller, J. J., Hoy, K., Farzan, F., and Daskalakis, Z. J. (2009). GABA and cortical inhibition in motor and non-motor regions using combined TMS-EEG: A time analysis. Clinical Neurophysiology, 120(9):1706-1710.

Fleming, M. K., Sorinola, I. O., Newham, D. J., Roberts-Lewis, S. F., and Bergmann, J. H. M. (2012). The effect of coil type and navigation on the reliability of transcranial magnetic stimulation. IEEE Transactions on Neural Systems and Rehabilitation Engineering, 20(5):617-625.

Forsgren, L., Bucht, G., Eriksson, S., and Bergmark, L. (1996). Incidence and clinical characterization of unprovoked seizures in adults: a prospective population-based study. Epilepsia, 37(3):224-229. 
Fox, P., Ingham, R., George, M. S., Mayberg, H., Ingham, J., Roby, J., et al. (1997). Imaging human intra-cerebral connectivity by PET during TMS. Neuroreport, 8(12):2787-2791.

Fuhr, P., Agostino, R., and Hallett, M. (1991). Spinal motor neuron excitability during the silent period after cortical stimulation. Electroencephalography and Clinical Neurophysiology/Evoked Potentials Section, 81(4):257-262.

Garvey, M. A., Ziemann, U., Becker, D. A., Barker, C. A., and Bartko, J. J. (2001). New graphical method to measure silent periods evoked by transcranial magnetic stimulation. Clinical neurophysiology, 112(8):1451-1460.

George, M. S., Wassermann, E. M., Williams, W. A., Callahan, A., Ketter, T. A., Basser, P., et al. (1995). Daily repetitive transcranial magnetic stimulation (rTMS) improves mood in depression. NeuroReport, 6(14):1853-1856.

Geut, I., Weenink, S., Knottnerus, I. L. H., and van Putten, M. J. A. M. (2017). Detecting interictal discharges in first seizure patients: ambulatory EEG or EEG after sleep deprivation? Seizure, 51:52-54.

Goldsworthy, M. R., Hordacre, B., and Ridding, M. C. (2016). Minimum number of trials required for withinand between-session reliability of TMS measures of corticospinal excitability. Neuroscience, 320:205-209.

Gonzalez, O. J. A., Aerde, K. I. v., Mansvelder, H. D., Pelt, J. v., and Ooyen, A. v. (2014). Inter-network interactions: Impact of connections between oscillatory neuronal networks on oscillation frequency and pattern. PLOS ONE, 9(7):e100899.

Grooms, J. K., Thompson, G. J., Pan, W.-J., Billings, J., Schumacher, E. H., Epstein, C. M., et al. (2017). Infraslow electroencephalographic and dynamic resting state network activity. Brain Connectivity, 7(5):265-280.

Groppa, S., Oliviero, A., Eisen, A., Quartarone, A., Cohen, L. G., Mall, V., et al. (2012). A practical guide to diagnostic transcranial magnetic stimulation: Report of an IFCN committee. Clinical Neurophysiology, 123(5):858-882.

Groppa, S., Siebner, H. R., Kurth, C., Stephani, U., and Siniatchkin, M. (2008). Abnormal response of motor cortex to photic stimulation in idiopathic generalized epilepsy. Epilepsia, 49(12):2022-2029.

Gugino, L. D., Romero, J. R., Aglio, L., Titone, D., Ramirez, M., Pascual-Leone, A., et al. (2001). Transcranial magnetic stimulation coregistered with MRI: a comparison of a guided versus blind stimulation technique and its effect on evoked compound muscle action potentials. Clinical neurophysiology, 112(10):17811792.

Hallett, M. (2000). Transcranial magnetic stimulation and the human brain. Nature, 406(6792):147-150.

Hamer, H. M. (2005). Motor cortex excitability in focal epilepsies not including the primary motor area - A TMS study. Brain, 128(4):811-818.

Hanajima, R., Ugawa, Y., Terao, Y., Sakai, K., Furubayashi, T., Machii, K., et al. (1998). Paired-pulse magnetic stimulation of the human motor cortex: differences among I waves. The Journal of Physiology, 509(2):607618.

Hattemer, K., Knake, S., Reis, J., Rochon, J., Oertel, W. H., Rosenow, F., et al. (2007). Excitability of the motor cortex during ovulatory and anovulatory cycles: a transcranial magnetic stimulation study. Clinical Endocrinology, 66(3):387-393.

Haug, B. A., Schönle, P. W., Knobloch, C., and Köhne, M. (1992). Silent period measurement revives as a valuable diagnostic tool with transcranial magnetic stimulation. Electroencephalography and Clinical Neurophysiology, 85(2):158-160.

Hauser, W. A., Annegers, J. F., and Kurland, L. T. (1993). Incidence of epilepsy and unprovoked seizures in Rochester, Minnesota: 1935-1984. Epilepsia, 34(3):453-458.

He, J. (2003). Slow oscillation in non-lemniscal auditory thalamus. The Journal of Neuroscience: The Official Journal of the Society for Neuroscience, 23(23):8281-8290. 
Heller, L. and van Hulsteyn, D. B. (1992). Brain stimulation using electromagnetic sources: theoretical aspects. Biophysical journal, 63(1):129-138.

Hermsen, A. M., Haag, A., Duddek, C., Balkenhol, K., Bugiel, H., Bauer, S., et al. (2016). Test-retest reliability of single and paired pulse transcranial magnetic stimulation parameters in healthy subjects. Journal of the Neurological Sciences, 362:209-216.

Hernandez-Pavon, J. C., Metsomaa, J., Mutanen, T., Stenroos, M., Mäki, H., Ilmoniemi, R. J., et al. (2012). Uncovering neural independent components from highly artifactual TMS-evoked EEG data. Journal of Neuroscience Methods, 209(1):144-157.

Herwig, U., Schönfeldt-Lecuona, C., Wunderlich, A. P., von Tiesenhausen, C., Thielscher, A., Walter, H., et al. (2001). The navigation of transcranial magnetic stimulation. Psychiatry Research, 108(2):123-131.

Hess, C. W., Mills, K. R., and Murray, N. M. (1986). Magnetic stimulation of the human brain: Facilitation of motor responses by voluntary contraction of ipsilateral and contralateral muscles with additional observations on an amputee. Neuroscience Letters, 71(2):235-240.

Hess, C. W., Mills, K. R., and Murray, N. M. (1987). Responses in small hand muscles from magnetic stimulation of the human brain. The Journal of physiology, 388:397.

Hiltunen, T., Kantola, J., Abou Elseoud, A., Lepola, P., Suominen, K., Starck, T., et al. (2014). Infra-slow EEG fluctuations are correlated with resting-state network dynamics in fMRI. The Journal of Neuroscience: The Official Journal of the Society for Neuroscience, 34(2):356-362.

Hsu, W.-Y., Cheng, C.-H., Lin, M.-W., Shih, Y.-H., Liao, K.-K., and Lin, Y.-Y. (2011). Antiepileptic effects of low frequency repetitive transcranial magnetic stimulation: A meta-analysis. Epilepsy Research, 96(3):231240.

Huber, R., Maki, H., Rosanova, M., Casarotto, S., Canali, P., Casali, A. G., et al. (2013). Human cortical excitability increases with time awake. Cerebral Cortex, 23(2):1-7.

Hufnagel, A., Elger, C. E., Marx, W., and Ising, A. (1990). Magnetic motor-evoked potentials in epilepsy: Effects of the disease and of anticonvulsant medication. Annals of neurology, 28(5):680-686.

Hughes, S. W., Lorincz, M. L., Parri, H. R., and Crunelli, V. (2011). Infraslow ( $<0.1 \mathrm{~Hz}$ ) oscillations in thalamic relay nuclei basic mechanisms and significance to health and disease states. Progress in Brain Research, 193:145-162.

Ilić, T. V., Meintzschel, F., Cleff, U., Ruge, D., Kessler, K. R., and Ziemann, U. (2002). Short-interval paired-pulse inhibition and facilitation of human motor cortex: the dimension of stimulus intensity. The Journal of Physiology, 545(1):153-167.

Ilmoniemi, R. J., Hernandez-Pavon, J. C., Mäkelä, N. N., Metsomaa, J., Mutanen, T. P., Stenroos, M., et al. (2015). Dealing with artifacts in TMS-evoked EEG. In Engineering in Medicine and Biology Society (EMBC), 201537 th Annual International Conference of the IEEE, pages 230-233. IEEE.

Ilmoniemi, R. J. and Kičić, D. (2010). Methodology for combined TMS and EEG. Brain Topography, 22(4):233248.

Ilmoniemi, R. J., Virtanen, J., Ruohonen, J., Karhu, J., Aronen, H. J., Näätänen, R., et al. (1997). Neuronal responses to magnetic stimulation reveal cortical reactivity and connectivity. Neuroreport, 8(16):35373540.

Inghilleri, M., Berardelli, A., Cruccu, G., and Manfredi, M. (1993). Silent period evoked by transcranial stimulation of the human cortex and cervicomedullary junction. The Journal of Physiology, 466:521.

Inghilleri, M., Berardelli, A., Marchetti, P., and Manfredi, M. (1996). Effects of diazepam, baclofen and thiopental on the silent period evoked by transcranial magnetic stimulation in humans. Experimental brain research, 109(3):467-472.

Inghilleri, M., Conte, A., Currà, A., Frasca, V., Lorenzano, C., and Berardelli, A. (2004). Ovarian hormones and cortical excitability. An rTMS study in humans. Clinical Neurophysiology: Official Journal of the International Federation of Clinical Neurophysiology, 115(5):1063-1068. 
Iscan, Z., Nazarova, M., Fedele, T., Blagovechtchenski, E., and Nikulin, V. V. (2016). Pre-stimulus alpha oscillations and inter-subject variability of motor evoked potentials in single- and paired-pulse TMS paradigms. Frontiers in Human Neuroscience, 10.

Jalinous, R. (1991). Technical and practical aspects of magnetic nerve stimulation. Journal of Clinical Neurophysiology: Official Publication of the American Electroencephalographic Society, 8(1):10-25.

Joo, E. Y., Kim, H.-J., Lim, Y.-H., Ji, K.-H., and Hong, S. B. (2010). Zonisamide changes unilateral cortical excitability in focal epilepsy patients. Journal of Clinical Neurology, 6(4):189.

Joo, E. Y., Kim, S. H., Seo, D. W., and Hong, S. B. (2008). Zonisamide decreases cortical excitability in patients with idiopathic generalized epilepsy. Clinical Neurophysiology, 119(6):1385-1392.

Julkunen, P., Säisänen, L., Danner, N., Niskanen, E., Hukkanen, T., Mervaala, E., et al. (2009). Comparison of navigated and non-navigated transcranial magnetic stimulation for motor cortex mapping, motor threshold and motor evoked potentials. Neurolmage, 44(3):790-795.

Julkunen, P., Säisänen, L., Hukkanen, T., Danner, N., and Könönen, M. (2012). Does second-scale intertrial interval affect motor evoked potentials induced by single-pulse transcranial magnetic stimulation? Brain Stimulation, 5(4):526-532.

Julkunen, P., Säisänen, L., Könönen, M., Vanninen, R., Kälviäinen, R., and Mervaala, E. (2013). TMSEEG reveals impaired intracortical interactions and coherence in Unverricht-Lundborg type progressive myoclonus epilepsy (EPM1). Epilepsy Research, 106(1-2):103-112.

Jung, N. H., Delvendahl, I., Kuhnke, N. G., Hauschke, D., Stolle, S., and Mall, V. (2010). Navigated transcranial magnetic stimulation does not decrease the variability of motor-evoked potentials. Brain Stimulation, 3(2):87-94.

Kallioniemi, E., Könönen, M., and Julkunen, P. (2015). Repeatability of functional anisotropy in navigated transcranial magnetic stimulation - coil-orientation versus response:. NeuroReport, 26(9):515-521.

Kammer, T., Beck, S., Thielscher, A., Laubis-Herrmann, U., and Topka, H. (2001). Motor thresholds in humans: A transcranial magnetic stimulation study comparing different pulse waveforms, current directions and stimulator types. Clinical neurophysiology, 112(2):250-258.

Kazis, D. A., Kimiskidis, V. K., Papagiannopoulos, S., Sotirakoglou, K., Divanoglou, D., Vlaikidis, N., et al. (2006). The effect of valproate on silent period and corticomotor excitability. Epileptic Disorders, 8(2):136-142.

Keil, J., Timm, J., SanMiguel, I., Schulz, H., Obleser, J., and Schonwiesner, M. (2014). Cortical brain states and corticospinal synchronization influence TMS-evoked motor potentials. Journal of Neurophysiology, 111(3):513-519.

Kerwin, L. J., Keller, C. J., Wu, W., Narayan, M., and Etkin, A. (2018). Test-retest reliability of transcranial magnetic stimulation EEG evoked potentials. Brain Stimulation, 11(3):536-544.

Kiers, L., Cros, D., Chiappa, K. H., and Fang, J. (1993). Variability of motor potentials evoked by transcranial magnetic stimulation. Electroencephalography and Clinical Neurophysiology, 89(6):415-423.

Kimiskidis, V., Papagiannopoulos, S., Sotirakoglou, K., Kazis, D. A., Kazis, A., and Mills, K. R. (2005). Silent period to transcranial magnetic stimulation: Construction and properties of stimulus-response curves in healthy volunteers. Experimental Brain Research, 163(1):21-31.

Kimiskidis, V. K. (2010). Transcranial magnetic stimulation for drug-resistant epilepsies: Rationale and clinical experience. European Neurology, 63(4):205-210.

Kimiskidis, V. K. (2016). Transcranial magnetic stimulation (TMS) coupled with electroencephalography (EEG): Biomarker of the future. Revue Neurologique, 172(2):123-126.

Kimiskidis, V. K., Papagiannopoulos, S., Kazis, D. A., Sotirakoglou, K., Vasiliadis, G., Zara, F., et al. (2006). Lorazepam-induced effects on silent period and corticomotor excitability. Experimental Brain Research, 173(4):603-611. 
Kimiskidis, V. K., Papagiannopoulos, S., Sotirakoglou, K., Kazis, D. A., Dimopoulos, G., Kazis, A., et al. (2004). The repeatability of corticomotor threshold measurements. Neurophysiologie Clinique/Clinical Neurophysiology, 34(6):259-266.

Kimiskidis, V. K., Tsimpiris, A., Ryvlin, P., Kalviainen, R., Koutroumanidis, M., Valentin, A., et al. (2017). TMS combined with EEG in genetic generalized epilepsy: A phase II diagnostic accuracy study. Clinical Neurophysiology, 128(2):367-381.

Kimiskidis, V. K., Valentin, A., and Kälviäinen, R. (2014). Transcranial magnetic stimulation for the diagnosis and treatment of epilepsy. Current Opinion in Neurology, 27(2):236-241.

King, M. A., Newton, M. R., Jackson, G. D., Fitt, G. J., Mitchell, L. A., Silvapulle, M. J., et al. (1998). Epileptology of the first-seizure presentation: a clinical, electroencephalographic, and magnetic resonance imaging study of 300 consecutive patients. The Lancet, 352(9133):1007-1011.

King, N. K. K., Kuppuswamy, A., Strutton, P. H., and Davey, N. J. (2006). Estimation of cortical silent period following transcranial magnetic stimulation using a computerised cumulative sum method. Journal of Neuroscience Methods, 150(1):96-104.

Klimpe, S., Behrang-Nia, M., Bott, M. C., and Werhahn, K. J. (2009). Recruitment of motor cortex inhibition differentiates between generalized and focal epilepsy. Epilepsy Research, 84(2-3):210-216.

Kobayashi, M. and Pascual-Leone, A. (2003). Transcranial magnetic stimulation in neurology. The Lancet Neurology, 2(3):145-156.

Komssi, S., Aronen, H. J., Huttunen, J., Kesäniemi, M., Soinne, L., Nikouline, V. V., et al. (2002). Ipsi-and contralateral EEG reactions to transcranial magnetic stimulation. Clinical Neurophysiology, 113(2):175184.

Komssi, S., Kähkönen, S., and Ilmoniemi, R. J. (2004). The effect of stimulus intensity on brain responses evoked by transcranial magnetic stimulation: TMS-Evoked Brain Responses. Human Brain Mapping, 21(3):154-164.

Komssi, S., Savolainen, P., Heiskala, J., and Kähkönen, S. (2007). Excitation threshold of the motor cortex estimated with transcranial magnetic stimulation electroencephalography. Neuroreport, 18(1):13-16.

Kopell, N., Ermentrout, G. B., Whittington, M. A., and Traub, R. D. (2000). Gamma rhythms and beta rhythms have different synchronization properties. Proceedings of the National Academy of Sciences of the United States of America, 97(4):1867-1872.

Korhonen, R. J., Hernandez-Pavon, J. C., Metsomaa, J., Mäki, H., Ilmoniemi, R. J., and Sarvas, J. (2011). Removal of large muscle artifacts from transcranial magnetic stimulation-evoked EEG by independent component analysis. Medical \& Biological Engineering \& Computing, 49(4):397-407.

Koutroumanidis, M., Arzimanoglou, A., Caraballo, R., Goyal, S., Kaminska, A., Laoprasert, P., et al. (2017). The role of EEG in the diagnosis and classification of the epilepsy syndromes: a tool for clinical practice by the ILAE Neurophysiology Task Force (Part 1). Epileptic Disorders, 19(3):233-298.

Kraskov, A., Kreuz, T., Andrzejak, R. G., Stögbauer, H., Nadler, W., and Grassberger, P. (2004). Extracting phases from aperiodic signals. arXiv preprint cond-mat/0409382.

Krumholz, A., Wiebe, S., Gronseth, G., Shinnar, S., Levisohn, P., Ting, T., et al. (2007). Practice parameter: Evaluating an apparent unprovoked first seizure in adults (an evidence-based review) - Report of the Quality Standards Subcommittee of the American Academy of Neurology and the American Epilepsy Society. Neurology, 69(21):1996-2007.

Kujirai, T., Caramia, M. D., Rothwell, J. C., Day, B. L., Thompson, P. D., Ferbert, A., et al. (1993). Corticocortical inhibition in human motor cortex. The Journal of physiology, 471:501.

Kundu, B., Johnson, J. S., and Postle, B. R. (2014). Prestimulation phase predicts the TMS-evoked response. Journal of Neurophysiology, 112(8):1885-1893. 
Kwan, P., Arzimanoglou, A., Berg, A. T., Brodie, M. J., Allen Hauser, W., Mathern, G., et al. (2009). Definition of drug resistant epilepsy: Consensus proposal by the ad hoc Task Force of the ILAE Commission on Therapeutic Strategies: Definition of Drug Resistant Epilepsy. Epilepsia, 51(6):1069-1077.

Kwan, P. and Brodie, M. J. (2000). Early identification of refractory epilepsy. New England Journal of Medicine, 342(5):314-319.

Kwan, P. and Brodie, M. J. (2001). Effectiveness of first antiepileptic drug. Epilepsia, 42(10):1255-1260.

Kähkönen, S., Komssi, S., Wilenius, J., and Ilmoniemi, R. J. (2005). Prefrontal transcranial magnetic stimulation produces intensity-dependent EEG responses in humans. Neurolmage, 24(4):955-960.

Kähkönen, S., Wilenius, J., Nikulin, V. V., Ollikainen, M., and Ilmoniemi, R. J. (2003). Alcohol reduces prefrontal cortical excitability in humans: A combined TMS and EEG study. Neuropsychopharmacology, 28(4):747754.

Lang, N., Hasan, A., Sueske, E., Paulus, W., and Nitsche, M. A. (2008). Cortical hypoexcitability in chronic smokers? A transcranial magnetic stimulation study. Neuropsychopharmacology, 33(10):2517-2523.

Lang, N., Rothkegel, H., Reiber, H., Hasan, A., Sueske, E., Tergau, F., et al. (2011). Circadian modulation of GABA-mediated cortical inhibition. Cerebral Cortex, 21(10):2299-2306.

Lee, H. W., Seo, H. J., Cohen, L. G., Bagic, A., and Theodore, W. H. (2005). Cortical excitability during prolonged antiepileptic drug treatment and drug withdrawal. Clinical Neurophysiology, 116(5):1105-1112.

Lee, J. H., Joo, E. Y., Seo, D. W., and Hong, S. B. (2015). Lateralizing cortical excitability in drug naïve patients with generalized or focal epilepsy. Journal of Epilepsy Research, 5(2):75-83.

Lefaucheur, J.-P. (2010). Why image-guided navigation becomes essential in the practice of transcranial magnetic stimulation. Neurophysiologie Clinique/Clinical Neurophysiology, 40(1):1-5.

Lefaucheur, J.-P., André-Obadia, N., Antal, A., Ayache, S. S., Baeken, C., Benninger, D. H., et al. (2014). Evidence-based guidelines on the therapeutic use of repetitive transcranial magnetic stimulation (rTMS). Clinical Neurophysiology, 125(11):2150-2206.

Liepert, J., Tegenthoff, M., and Malin, J. P. (1995). Changes of cortical motor area size during immobilization. Electroencephalography and Clinical Neurophysiology, 97(6):382-386.

Lin, L. I.-K. (1989). A concordance correlation coefficient to evaluate reproducibility. Biometrics, 45(1):255.

Lioumis, P., Kičić, D., Savolainen, P., Mäkelä, J. P., and Kähkönen, S. (2009). Reproducibility of TMS-Evoked EEG responses. Human Brain Mapping, 30(4):1387-1396.

Lörincz, M. L., Geall, F., Bao, Y., Crunelli, V., and Hughes, S. W. (2009). ATP-dependent infra-slow $(<0.1 \mathrm{~Hz})$ oscillations in thalamic networks. PloS One, 4(2):e4447.

Maccabee, P. J., Amassian, V. E., Eberle, L. P., and Cracco, R. Q. (1993). Magnetic coil stimulation of straight and bent amphibian and mammalian peripheral nerve in vitro: Locus of excitation. Journal of Physiology, 460:201-219.

Maccabee, P. J., Nagarajan, S. S., Amassian, V. E., Durand, D. M., Szabo, A. Z., Ahad, A. B., et al. (1998). Influence of pulse sequence, polarity and amplitude on magnetic stimulation of human and porcine peripheral nerve. The Journal of physiology, 513(2):571-585.

Macdonell, R. A. L., King, M. A., Newton, M. R., Curatolo, J. M., Reutens, D. C., and Berkovic, S. F. (2001). Prolonged cortical silent period after transcranial magnetic stimulation in generalized epilepsy. Neurology, 57(4):706-708.

Maeda, F., Gangitano, M., Thall, M., and Pascual-Leone, A. (2002). Inter-and intra-individual variability of paired-pulse curves with transcranial magnetic stimulation (TMS). Clinical neurophysiology, 113(3):376382.

Manganotti, P., Bongiovanni, L. G., Zanette, G., Turazzini, M., and Fiaschi, A. (1999). Cortical excitability in patients after loading doses of lamotrigine: A study with magnetic brain stimulation. Epilepsia, 40(3):316321. 
Maris, E. and Oostenveld, R. (2007). Nonparametric statistical testing of EEG- and MEG-data. Journal of Neuroscience Methods, 164(1):177-190.

Mars, R. B., Bestmann, S., Rothwell, J. C., and Haggard, P. (2007). Effects of motor preparation and spatial attention on corticospinal excitability in a delayed-response paradigm. Experimental Brain Research, 182(1):125-129.

Massimini, M. (2005). Breakdown of cortical effective connectivity during sleep. Science, 309(5744):22282232.

Mathias, J. P., Barsi, G. I., van de Ruit, M., and Grey, M. J. (2014). Rapid acquisition of the transcranial magnetic stimulation stimulus response curve. Brain Stimulation, 7(1):59-65.

McCormick, D. A. and Contreras, D. (2001). On the cellular and network bases of epileptic seizures. Annual review of physiology, 63(1):815-846.

McDonnell, M. N., Orekhov, Y., and Ziemann, U. (2006). The role of GABAB receptors in intracortical inhibition in the human motor cortex. Experimental Brain Research, 173(1):86-93.

McNamara, J. O. (1994). Cellular and molecular basis of epilepsy. The journal of neuroscience, 14(6):34133425.

Michelucci, R., Passarelli, D., Riguzzi, P., Buzzi, A. M., Gardella, E., and Tassinari, C. A. (1996). Transcranial magnetic stimulation in partial epilepsy: Drug-induced changes of motor excitability. Acta neurologica scandinavica, 94(1):24-30.

Mills, K. R. and Nithi, K. A. (1997). Corticomotor threshold to magnetic stimulation: Normal values and repeatability. Muscle and Nerve, 20(5):570-576.

Monto, S., Palva, S., Voipio, J., and Palva, J. M. (2008). Very slow EEG fluctuations predict the dynamics of stimulus detection and oscillation amplitudes in humans. Journal of Neuroscience, 28(33):8268-8272.

Moshé, S. L., Perucca, E., Ryvlin, P., and Tomson, T. (2015). Epilepsy: new advances. The Lancet, 385(9971):884-898.

Mutanen, T., Mäki, H., and Ilmoniemi, R. J. (2013). The effect of stimulus parameters on TMS-EEG muscle artifacts. Brain Stimulation, 6(3):371-376.

Mäki, H. and Ilmoniemi, R. J. (2010a). EEG oscillations and magnetically evoked motor potentials reflect motor system excitability in overlapping neuronal populations. Clinical Neurophysiology, 121(4):492-501.

Mäki, H. and Ilmoniemi, R. J. (2010b). The relationship between peripheral and early cortical activation induced by transcranial magnetic stimulation. Neuroscience Letters, 478(1):24-28.

Möller, C., Arai, N., Lücke, J., and Ziemann, U. (2009). Hysteresis effects on the input-output curve of motor evoked potentials. Clinical Neurophysiology, 120(5):1003-1008.

Müller-Dahlhaus, J. F. M., Liu, Y., and Ziemann, U. (2008). Inhibitory circuits and the nature of their interactions in the human motor cortex - a pharmacological TMS study: Inhibitory circuits and their interactions in human motor cortex. The Journal of Physiology, 586(2):495-514.

Nakamura, H., Kitagawa, H., Kawaguchi, Y., and Tsuji, H. (1997). Intracortical facilitation and inhibition after transcranial magnetic stimulation in conscious humans. The Journal of Physiology, 498 ( Pt 3):817-823.

Nei, M. and Bagla, R. (2007). Seizure-related injury and death. Current neurology and neuroscience reports, 7(4):335-341.

Nezu, A., Kimura, S., Ohtsuki, N., and Tanaka, M. (1997). Transcranial magnetic stimulation in benign childhood epilepsy with centro-temporal spikes. Brain and Development, 19(2):134-137.

Ng, B. S. W., Logothetis, N. K., and Kayser, C. (2013). EEG phase patterns reflect the selectivity of neural firing. Cerebral Cortex, 23(2):389-398.

Ngugi, A. K., Bottomley, C., Kleinschmidt, I., Sander, J. W., and Newton, C. R. (2010). Estimation of the burden of active and life-time epilepsy: A meta-analytic approach: Estimation of the Burden of Epilepsy. Epilepsia, 51(5):883-890. 
Ngugi, A. K., Kariuki, S. M., Bottomley, C., Kleinschmidt, I., Sander, J. W., and Newton, C. R. (2011). Incidence of epilepsy: a systematic review and meta-analysis. Neurology, 77(10):1005-1012.

$\mathrm{Ni}$, Z. and Chen, R. (2015). Transcranial magnetic stimulation to understand pathophysiology and as potential treatment for neurodegenerative diseases. Translational Neurodegeneration, 4(1).

Nickerson, C. A. E. (1997). A note on "A concordance correlation coefficient to evaluate reproducibility". Biometrics, 53(4):1503.

Niehaus, L., Meyer, B.-U., and Weyh, T. (2000). Influence of pulse configuration and direction of coil current on excitatory effects of magnetic motor cortex and nerve stimulation. Clinical neurophysiology, 111(1):75-80.

Nilsson, J., Panizza, M., and Arieti, P. (1997). Computer-aided determination of the silent period. Journal of clinical neurophysiology, 14(2):136-143.

Oldfield, R. C. (1971). The assessment and analysis of handedness: the Edinburgh inventory. Neuropsychologia, 9(1):97-113.

Oliveira, B., Mitjans, M., Nitsche, M. A., Kuo, M.-F., and Ehrenreich, H. (2018). Excitation-inhibition dysbalance as predictor of autistic phenotypes. Journal of Psychiatric Research, 104:96-99.

Opie, G. M., Rogasch, N. C., Goldsworthy, M. R., Ridding, M. C., and Semmler, J. G. (2017). Investigating TMSEEG indices of long-interval intracortical inhibition at different interstimulus intervals. Brain Stimulation, 10(1):65-74.

Opie, G. M., Sidhu, S. K., Rogasch, N. C., Ridding, M. C., and Semmler, J. G. (2018). Cortical inhibition assessed using paired-pulse TMS-EEG is increased in older adults. Brain Stimulation, 11(3):545-557.

Orth, M., Amann, B., Ratnaraj, N., Patsalos, P. N., and Rothwell, J. C. (2005). Caffeine has no effect on measures of cortical excitability. Clinical Neurophysiology, 116(2):308-314.

Orth, M. and Rothwell, J. C. (2004). The cortical silent period: Intrinsic variability and relation to the waveform of the transcranial magnetic stimulation pulse. Clinical Neurophysiology, 115(5):1076-1082.

Orth, M., Snijders, A. H., and Rothwell, J. C. (2003). The variability of intracortical inhibition and facilitation. Clinical Neurophysiology, 114(12):2362-2369.

Ortu, E., Deriu, F., Suppa, A., Tolu, E., and Rothwell, J. C. (2008). Effects of volitional contraction on intracortical inhibition and facilitation in the human motor cortex: Intracortical excitability during volitional contraction. The Journal of Physiology, 586(21):5147-5159.

Parri, H. R. and Crunelli, V. (2001). Pacemaker calcium oscillations in thalamic astrocytes in situ. Neuroreport, 12(18):3897-3900.

Parri, H. R., Gould, T. M., and Crunelli, V. (2001). Spontaneous astrocytic Ca2+ oscillations in situ drive NMDARmediated neuronal excitation. Nature Neuroscience, 4(8):803-812.

Pascual-Leone, A., Gates, J. R., and Dhuna, A. (1991). Induction of speech arrest and counting errors with rapid-rate transcranial magnetic stimulation. Neurology, 41(5):697-702.

Pascual-Leone, A. and Hallett, M. (1994). Induction of errors in a delayed response task by repetitive transcranial magnetic stimulation of the dorsolateral prefrontal cortex. NeuroReport, 5(18):2517-2520.

Pascual-Leone, A. and Torres, F. (1993). Plasticity of the sensorimotor cortex representation of the reading finger in Braille readers. Brain, 116(1):39-52.

Pascual-Leone, A., Valls-Solé, J., Wassermann, E. M., and Hallett, M. (1994). Responses to rapid-rate transcranial magnetic stimulation of the human motor cortex. Brain, 117(4):847-858.

Patton, H. D. and Amassian, V. E. (1954). Single-and multiple-unit analysis of cortical stage of pyramidal tract activation. Journal of Neurophysiology, 17(4):345-363.

Paus, T., Jech, R., Thompson, C. J., Comeau, R., Peters, T., and Evans, A. C. (1997). Transcranial magnetic stimulation during positron emission tomography: A new method for studying connectivity of the human cerebral cortex. The Journal of neuroscience, 17(9):3178-3184. 
Paus, T., Sipila, P. K., and Strafella, A. P. (2001). Synchronization of neuronal activity in the human primary motor cortex by transcranial magnetic stimulation: An EEG study. Journal of Neurophysiology, 86(4):19831990.

Pawley, A. D., Chowdhury, F. A., Tangwiriyasakul, C., Ceronie, B., Elwes, R. D. C., Nashef, L., et al. (2017). Cortical excitability correlates with seizure control and epilepsy duration in chronic epilepsy. Annals of Clinical and Translational Neurology, 4(2):87-97.

Pearce, A. J., Clark, R. A., and Kidgell, D. J. (2013). A comparison of two methods in acquiring stimulusresponse curves with transcranial magnetic stimulation. Brain Stimulation, 6(3):306-309.

Pellicciari, M. C., Miniussi, C., Ferrari, C., Koch, G., and Bortoletto, M. (2016). Ongoing cumulative effects of single TMS pulses on corticospinal excitability: An intra- and inter-block investigation. Clinical Neurophysiology, 127(1):621-628.

Pesaran, B., Vinck, M., Einevoll, G. T., Sirota, A., Fries, P., Siegel, M., et al. (2018). Investigating large-scale brain dynamics using field potential recordings: analysis and interpretation. Nature Neuroscience, 21(7):903919.

Pierantozzi, M., Grazia Marciani, M., Giuseppina Palmieri, M., Brusa, L., Galati, S., Donatella Caramia, M., et al. (2004). Effect of Vigabatrin on motor responses to transcranial magnetic stimulation. Brain Research, 1028(1):1-8.

Pitcher, J. B., Ogston, K. M., and Miles, T. S. (2003). Age and sex differences in human motor cortex inputoutput characteristics. The Journal of Physiology, 546(2):605-613.

Premoli, I., Castellanos, N., Rivolta, D., Belardinelli, P., Bajo, R., Zipser, C., et al. (2014a). TMS-EEG Signatures of GABAergic Neurotransmission in the Human Cortex. Journal of Neuroscience, 34(16):5603-5612.

Premoli, I., Király, J., Müller-Dahlhaus, F., Zipser, C. M., Rossini, P., Zrenner, C., et al. (2018). Short-interval and long-interval intracortical inhibition of TMS-evoked EEG potentials. Brain Stimulation, 11(4):818-827.

Premoli, I., Rivolta, D., Espenhahn, S., Castellanos, N., Belardinelli, P., Ziemann, U., et al. (2014b). Characterization of GABAB-receptor mediated neurotransmission in the human cortex by paired-pulse TMS-EEG. Neurolmage, 103:152-162.

Puri, V., Sajan, P. M., Chowdhury, V., and Chaudhry, N. (2013). Cortical excitability in drug naive juvenile myoclonic epilepsy. Seizure, 22(8):662-669.

Qi, F., Wu, A. D., and Schweighofer, N. (2011). Fast estimation of transcranial magnetic stimulation motor threshold. Brain Stimulation, 4(1):50-57.

Quintas, R., Raggi, A., Giovannetti, A. M., Pagani, M., Sabariego, C., Cieza, A., et al. (2012). Psychosocial difficulties in people with epilepsy: A systematic review of literature from 2005 until 2010. Epilepsy \& Behavior, 25(1):60-67.

Raffin, E., Pellegrino, G., Di Lazzaro, V., Thielscher, A., and Siebner, H. R. (2015). Bringing transcranial mapping into shape: Sulcus-aligned mapping captures motor somatotopy in human primary motor hand area. Neurolmage, 120:164-175.

RCore-Team (2015). R: a language and environment for statistical computing.

Reutens, D. C. and Berkovic, S. F. (1992). Increased cortical excitability in generalised epilepsy demonstrated with transcranial magnetic stimulation. The Lancet, 339(8789):362-363.

Reutens, D. C., Berkovic, S. F., Macdonell, R. A. L., and Bladin, P. F. (1993). Magnetic stimulation of the brain in generalized epilepsy: Reversal of cortical hyperexcitability by anticonvulsants. Annals of neurology, 34(3):351-355.

Rogasch, N. C., Daskalakis, Z. J., and Fitzgerald, P. B. (2013). Mechanisms underlying long-interval cortical inhibition in the human motor cortex: a TMS-EEG study. Journal of Neurophysiology, 109(1):89-98.

Rogasch, N. C. and Fitzgerald, P. B. (2013). Assessing cortical network properties using TMS-EEG. Human Brain Mapping, 34(7):1652-1669. 
Rogasch, N. C., Sullivan, C., Thomson, R. H., Rose, N. S., Bailey, N. W., Fitzgerald, P. B., et al. (2017). Analysing concurrent transcranial magnetic stimulation and electroencephalographic data: A review and introduction to the open-source TESA software. Neurolmage, 147:934-951.

Rogasch, N. C., Thomson, R. H., Farzan, F., Fitzgibbon, B. M., Bailey, N. W., Hernandez-Pavon, J. C., et al. (2014). Removing artefacts from TMS-EEG recordings using independent component analysis: Importance for assessing prefrontal and motor cortex network properties. Neurolmage, 101:425-439.

Rogawski, M. A. and Löscher, W. (2004). The neurobiology of antiepileptic drugs. Nature Reviews Neuroscience, 5(7):553-564.

Roger, J., Dreifuss, F. E., Martinez-Lage, M., Munari, C., Porter, R. J., Seino, M., et al. (1989). Proposal for revised classification of epilepsies and epileptic syndromes. Epilepsia, 30(4):389-399.

Roick, H., Von Giesen, H. J., and Benecke, R. (1993). On the origin of the postexcitatory inhibition seen after transcranial magnetic brain stimulation in awake human subjects. Experimental Brain Research, 94(3):489-498.

Rosenow, F., Klein, K. M., and Hamer, H. M. (2015). Non-invasive EEG evaluation in epilepsy diagnosis. Expert Review of Neurotherapeutics, 15(4):425-444.

Rossi, S., Hallett, M., Rossini, P. M., and Pascual-Leone, A. (2009). Safety, ethical considerations, and application guidelines for the use of transcranial magnetic stimulation in clinical practice and research. Clinical Neurophysiology, 120(12):2008-2039.

Rossi, S., Hallett, M., Rossini, P. M., and Pascual-Leone, A. (2011). Screening questionnaire before TMS: an update. Clinical Neurophysiology: Official Journal of the International Federation of Clinical Neurophysiology, 122(8):1686.

Rossini, P. M., Barker, A. T., Berardelli, A., Caramia, M. D., Caruso, G., Cracco, R. Q., et al. (1994). Noninvasive electrical and magnetic stimulation of the brain, spinal cord and roots: Basic principles and procedures for routine clinical application. Report of an IFCN committee. Electroencephalography and clinical neurophysiology, 91(2):79-92.

Rossini, P. M., Burke, D., Chen, R., Cohen, L. G., Daskalakis, Z., Di lorio, R., et al. (2015). Non-invasive electrical and magnetic stimulation of the brain, spinal cord, roots and peripheral nerves: basic principles and procedures for routine clinical and research application. An updated report from an IFCN Committee. Clinical Neurophysiology, 126(6):1071-1107.

Roth, B. J., Saypol, J. M., Hallett, M., and Cohen, L. G. (1991). A theoretical calculation of the electric field induced in the cortex during magnetic stimulation. Electroencephalography and Clinical Neurophysiology/Evoked Potentials Section, 81(1):47-56.

Roth, Y., Zangen, A., and Hallett, M. (2002). A coil design for transcranial magnetic stimulation of deep brain regions. Journal of Clinical Neurophysiology, 19(4):361-370.

Rothwell, J. C. (1997). Techniques and mechanisms of action of transcranial stimulation of the human motor cortex. Journal of neuroscience methods, 74(2):113-122.

Roy Choudhury, K., Boyle, L., Burke, M., Lombard, W., Ryan, S., and McNamara, B. (2011). Intra subject variation and correlation of motor potentials evoked by transcranial magnetic stimulation. Irish Journal of Medical Science, 180(4):873-880.

Rösler, K. M., Hess, C. W., Heckmann, R., and Ludin, H. P. (1989). Significance of shape and size of the stimulating coil in magnetic stimulation of the human motor cortex. Neuroscience letters, 100(1-3):347352.

Sander, J. W. and Shorvon, S. D. (1996). Epidemiology of the epilepsies. Journal of neurology, neurosurgery, and psychiatry, 61(5):433.

Sanger, T. D., Garg, R. R., and Chen, R. (2001). Interactions between two different inhibitory systems in the human motor cortex. The Journal of Physiology, 530(2):307-317. 
Sauseng, P., Klimesch, W., Gerloff, C., and Hummel, F. C. (2009). Spontaneous locally restricted EEG alpha activity determines cortical excitability in the motor cortex. Neuropsychologia, 47(1):284-288.

Scheffer, I. E., Berkovic, S., Capovilla, G., Connolly, M. B., French, J., Guilhoto, L., et al. (2017). ILAE classification of the epilepsies: Position paper of the ILAE Commission for Classification and Terminology. Epilepsia, 58(4):512-521.

Schmidt, S., Bathe-Peters, R., Fleischmann, R., Rönnefarth, M., Scholz, M., and Brandt, S. A. (2015). Nonphysiological factors in navigated TMS studies; Confounding covariates and valid intracortical estimates: Physical Parameters in nTMS, Variance and Validity. Human Brain Mapping, 36(1):40-49.

Schulz, H., Ubelacker, T., Keil, J., Muller, N., and Weisz, N. (2014). Now I am ready-now I am not: The influence of pre-TMS oscillations and corticomuscular coherence on motor-evoked potentials. Cerebral Cortex, 24(7):1708-1719.

Schwartzkroin, P. A. (1994). Cellular electrophysiology of human epilepsy. Epilepsy research, 17(3):185-192.

Schwenkreis, P., Witscher, K., Janssen, F., Addo, A., Dertwinkel, R., Zenz, M., et al. (1999). Influence of the $\mathrm{N}$-methyl-D-aspartate antagonist memantine on human motor cortex excitability. Neuroscience letters, 270(3):137-140.

Schönfeldt-Lecuona, C., Thielscher, A., Freudenmann, R. W., Kron, M., Spitzer, M., and Herwig, U. (2005). Accuracy of stereotaxic positioning of transcranial magnetic stimulation. Brain Topography, 17(4):253259.

Searle, S. R., Speed, F. M., and Milliken, G. A. (1980). Population marginal means in the linear model: An alternative to Least Squares Means. The American Statistician, 34(4):216-221.

Seyal, M., Masuoka, L. K., and Browne, J. K. (1992). Suppression of cutaneous perception by magnetic pulse stimulation of the human brain. Electroencephalography and Clinical Neurophysiology/Evoked Potentials Section, 85(6):397-401.

Seyal, M., Ro, T., and Rafal, R. (1995). Increased sensitivity to ipsilateral cutaneous stimuli following transcranial magnetic stimulation of the parietal lobe. Annals of neurology, 38(2):264-267.

Shafi, M. M., Vernet, M., Klooster, D., Chu, C. J., Boric, K., Barnard, M. E., et al. (2015). Physiological consequences of abnormal connectivity in a developmental epilepsy: Cortical Connectivity. Annals of Neurology, 77(3):487-503.

Shimizu, T., Filippi, M. M., Palmieri, M. G., Oliveri, M., Vernieri, F., Pasqualetti, P., et al. (1999). Modulation of intracortical excitability for different muscles in the upper extremity: paired magnetic stimulation study with focal versus non-focal coils. Clinical neurophysiology, 110(3):575-581.

Shrout, P. E. and Fleiss, J. L. (1979). Intraclass correlations: Uses in assessing rater reliability. Psychological Bulletin, 86(2):420-428.

Siebner, H. R., Dressnandt, J., Auer, C., and Conrad, B. (1998). Continuous intrathecal baclofen infusions induced a marked increase of the transcranially evoked silent period in a patient with generalized dystonia. Muscle \& Nerve, 21(9):1209-1212.

Silbert, B. I., Heaton, A. E., Cash, R. F., James, I., Dunne, J. W., Lawn, N. D., et al. (2015). Evidence for an excitatory GABAA response in human motor cortex in idiopathic generalised epilepsy. Seizure, 26:36-42.

Silvanto, J. and Pascual-Leone, A. (2008). State-dependency of transcranial magnetic stimulation. Brain Topography, 21(1):1-10.

Smith, M. J., Adams, L. F., Schmidt, P. J., Rubinow, D. R., and Wassermann, E. M. (2002). Effects of ovarian hormones on human cortical excitability. Annals of Neurology, 51(5):599-603.

Smith, M. J., Keel, J. C., Greenberg, B. D., Adams, L. F., Schmidt, P. J., Rubinow, D. A., et al. (1999). Menstrual cycle effects on cortical excitability. Neurology, 53(9):2069-2069.

Smith, S. J. M. (2005). EEG in the diagnosis, classification, and management of patients with epilepsy. Journal of Neurology, Neurosurgery \& Psychiatry, 76(suppl_2):ii2-ii7. 
Sommer, M., Alfaro, A., Rummel, M., Speck, S., Lang, N., Tings, T., et al. (2006). Half sine, monophasic and biphasic transcranial magnetic stimulation of the human motor cortex. Clinical Neurophysiology, $117(4): 838-844$.

Sparing, R., Buelte, D., Meister, I. G., Pauš, T., and Fink, G. R. (2008). Transcranial magnetic stimulation and the challenge of coil placement: A comparison of conventional and stereotaxic neuronavigational strategies. Human Brain Mapping, 29(1):82-96.

Sparing, R., Hesse, M. D., and Fink, G. R. (2010). Neuronavigation for transcranial magnetic stimulation (TMS): Where we are and where we are going. Cortex, 46(1):118-120.

Stefan, K., Kunesch, E., Cohen, L. G., Benecke, R., and Classen, J. (2000). Induction of plasticity in the human motor cortex by paired associative stimulation. Brain, 123(3):572-584.

Stern, W. M., Desikan, M., Hoad, D., Jaffer, F., Strigaro, G., Sander, J. W., et al. (2016). Spontaneously fluctuating motor cortex excitability in alternating hemiplegia of childhood: A transcranial magnetic stimulation study. PloS One, 11(3):e0151667.

Stern, W. M., Sander, J. W., Rothwell, J. C., and Sisodiya, S. M. (2017). Impaired intracortical inhibition demonstrated in vivo in people with Dravet syndrome. Neurology, 88(17):1659-1665.

Stetkarova, I. and Kofler, M. (2013). Differential effect of baclofen on cortical and spinal inhibitory circuits. Clinical Neurophysiology, 124(2):339-345.

Strigaro, G., Falletta, L., Varrasi, C., Rothwell, J. C., and Cantello, R. (2015). Overactive visuomotor connections underlie the photoparoxysmal response. A TMS study. Epilepsia, 56(11):1828-1835.

Strigaro, G., Prandi, P., Varrasi, C., Monaco, F., and Cantello, R. (2012). Defective visual inhibition in photosensitive idiopathic generalized epilepsy. Epilepsia, 53(4):695-704.

Suppa, A., Berardelli, A., Brancati, F., Marianetti, M., Barrano, G., Mina, C., et al. (2009). Clinical, neuropsychological, neurophysiologic, and genetic features of a new Italian pedigree with familial cortical myoclonic tremor with epilepsy. Epilepsia, 50(5):1284-1288.

Tassinari, C. A., Cincotta, M., Zaccara, G., and Michelucci, R. (2003). Transcranial magnetic stimulation and epilepsy. Clinical Neurophysiology, 114(5):777-798.

Tataroglu, C., Ozkiziltan, S., and Baklan, B. (2004). Motor cortical thresholds and cortical silent periods in epilepsy. Seizure, 13(7):481-485.

ter Braack, E. M., de Goede, A. A., and van Putten, M. J. A. M. (2018). Resting motor threshold, MEP and TEP variability during daytime. Brain Topography.

ter Braack, E. M., de Jonge, B., and van Putten, M. J. A. M. (2013a). Reduction of TMS induced artifacts in EEG using principal component analysis. IEEE Transactions on Neural Systems and Rehabilitation Engineering, 21(3):376-382.

ter Braack, E. M., de Vos, C. C., and van Putten, M. J. A. M. (2013b). Masking the auditory evoked potential in TMS-EEG: A comparison of various methods. Brain Topography, 28(3):520-528.

ter Braack, E. M., Koopman, A.-W. E., and van Putten, M. J. A. M. (2016). Early TMS evoked potentials in epilepsy: A pilot study. Clinical Neurophysiology, 127(9):3025-3032.

Tranulis, C., Guéguen, B., Pham-Scottez, A., Vacheron, M. N., Cabelguen, G., Costantini, A., et al. (2006). Motor threshold in transcranial magnetic stimulation: Comparison of three estimation methods. Neurophysiologie Clinique, 36(1):1-7.

Valentin, A., Arunachalam, R., Mesquita-Rodrigues, A., Garcia Seoane, J. J., Richardson, M. P., Mills, K. R., et al. (2008). Late EEG responses triggered by transcranial magnetic stimulation (TMS) in the evaluation of focal epilepsy. Epilepsia, 49(3):470-480.

Vallence, A.-M., Smalley, E., Drummond, P. D., and Hammond, G. R. (2017). Long-interval intracortical inhibition is asymmetric in young but not older adults. Journal of Neurophysiology, 118(3):1581-1590. 
Valls-Solé, J., Pascual-Leone, A., Wassermann, E. M., and Hallett, M. (1992). Human motor evoked responses to paired transcranial magnetic stimuli. Electroencephalography and Clinical Neurophysiology, 85(6):355364.

Valzania, F., Quatrale, R., Strafella, A. P., Bombardi, R., Santangelo, M., Tassinari, C. A., et al. (1994). Pattern of motor evoked response to repetitive transcranial magnetic stimulation. Electroencephalography and Clinical Neurophysiology/Evoked Potentials Section, 93(4):312-317.

van Putten, M. J. A. M., Tjepkema-Cloostermans, M. C., and Hofmeijer, J. (2015). Infraslow EEG activity modulates cortical excitability in postanoxic encephalopathy. Journal of Neurophysiology, 113(9):32563267.

van Strien, J. W. (1992). Classificatie van links-en rechtshandige proefpersonen [Classification of left- and right-handed research participants]. Nederlands tijdschrift voor de psychologie, 47:88-92.

van Strien, J. W. (2003). The Dutch handedness questionnaire.

Vanhatalo, S., Palva, J. M., Holmes, M. D., Miller, J. W., Voipio, J., and Kaila, K. (2004). Infraslow oscillations modulate excitability and interictal epileptic activity in the human cortex during sleep. Proceedings of the National Academy of Sciences of the United States of America, 101(14):5053-5057.

Varnava, A., Stokes, M. G., and Chambers, C. D. (2011). Reliability of the 'observation of movement' method for determining motor threshold using transcranial magnetic stimulation. Journal of Neuroscience Methods, 201(2):327-332.

Varrasi, C., Civardi, C., Boccagni, C., Cecchin, M., Vicentini, R., Monaco, F., et al. (2004). Cortical excitability in drug-naive patients with partial epilepsy - A cross-sectional study. Neurology, 63(11):2051-2055.

Vaseghi, B., Zoghi, M., and Jaberzadeh, S. (2015). Inter-pulse interval affects the size of single-pulse TMSinduced motor evoked potentials: a reliability study. Basic and clinical neuroscience, 6(1):44.

Velissaris, S. L., Wilson, S. J., Saling, M. M., Newton, M. R., and Berkovic, S. F. (2007). The psychological impact of a newly diagnosed seizure: Losing and restoring perceived control. Epilepsy \& Behavior, 10(2):223-233.

Voipio, J., Tallgren, P., Heinonen, E., Vanhatalo, S., and Kaila, K. (2003). Millivolt-scale DC shifts in the human scalp EEG: evidence for a nonneuronal generator. Journal of Neurophysiology, 89(4):2208-2214.

Wassermann, E., Epstein, C., and Ziemann, U. (2008). Oxford Handbook of Transcranial Stimulation. OUP Oxford.

Wassermann, E. M. (2002). Variation in the response to transcranial magnetic brain stimulation in the general population. Clinical Neurophysiology, 113(7):1165-1171.

Wassermann, E. M., Samii, A., Mercuri, B., Ikoma, K., Oddo, D., Grill, S. E., et al. (1996). Responses to paired transcranial magnetic stimuli in resting, active, and recently activated muscles. Experimental Brain Research, 109(1):158-163.

Werhahn, K. J., Kunesch, E., Noachtar, S., Benecke, R., and Classen, J. (1999). Differential effects on motorcortical inhibition induced by blockade of GABA uptake in humans. The Journal of physiology, 517(2):591-597.

Werhahn, K. J., Lieber, J., Classen, J., and Noachtar, S. (2000). Motor cortex excitability in patients with focal epilepsy. Epilepsy research, 41(2):179-189.

Wu, W., Keller, C. J., Rogasch, N. C., Longwell, P., Shpigel, E., Rolle, C. E., et al. (2018). ARTIST: A fully automated artifact rejection algorithm for single-pulse TMS-EEG data. Human Brain Mapping, 39(4):1607-1625.

Zarkowski, P., Shin, C. J., Dang, T., Russo, J., and Avery, D. (2006). EEG and the variance of motor evoked potential amplitude. Clinical EEG and Neuroscience, 37(3):247-251.

Ziemann, U. (2004). TMS and drugs. Clinical Neurophysiology, 115(8):1717-1729.

Ziemann, U. (2017). Thirty years of transcranial magnetic stimulation: where do we stand? Experimental Brain Research, 235(4):973-984. 
Ziemann, U., Chen, R., Cohen, L. G., and Hallett, M. (1998). Dextromethorphan decreases the excitability of the human motor cortex. Neurology, 51(5):1320-1324.

Ziemann, U., Lönnecker, S., and Paulus, W. (1995). Inhibition of human motor cortex by ethanol - A transcranial magnetic stimulation study. Brain, 118(6):1437-1446.

Ziemann, U., Lönnecker, S., Steinhoff, B. J., and Paulus, W. (1996a). Effects of antiepileptic drugs on motor cortex excitability in humans: a transcranial magnetic stimulation study. Annals of Neurology: Official Journal of the American Neurological Association and the Child Neurology Society, 40(3):367-378.

Ziemann, U., Netz, J., Szelényi, A., and Hömberg, V. (1993). Spinal and supraspinal mechanisms contribute to the silent period in the contracting soleus muscle after transcranial magnetic stimulation of human motor cortex. Neuroscience Letters, 156(1-2):167-171.

Ziemann, U., Reis, J., Schwenkreis, P., Rosanova, M., Strafella, A., Badawy, R. A. B., et al. (2015). TMS and drugs revisited 2014. Clinical Neurophysiology, 126(10):1847-1868.

Ziemann, U., Rothwell, J. C., and Ridding, M. C. (1996b). Interaction between intracortical inhibition and facilitation in human motor cortex. The Journal of physiology, 496(Pt 3):873.

Zoghi, M., Vaseghi, B., Bastani, A., Jaberzadeh, S., and Galea, M. P. (2015). The effects of sex hormonal fluctuations during menstrual cycle on cortical excitability and manual dexterity (a pilot study). PloS one, 10(8):e0136081.

Zrenner, C., Belardinelli, P., Müller-Dahlhaus, F., and Ziemann, U. (2016). Closed-loop neuroscience and noninvasive brain stimulation: A tale of two loops. Frontiers in Cellular Neuroscience, 10.

Zrenner, C., Desideri, D., Belardinelli, P., and Ziemann, U. (2018). Real-time EEG-defined excitability states determine efficacy of TMS-induced plasticity in human motor cortex. Brain Stimulation, 11(2):374-389. 



\section{List of abbreviations}

$\begin{array}{ll}\text { ADM } & \text { abductor digiti minimi } \\ \text { APB } & \text { abductor pollicis brevis } \\ \text { AED(s) } & \text { anti-epileptic drug(s) } \\ \text { aMT } & \text { active motor threshold } \\ \text { CR } & \text { conditioning response } \\ \text { CSP } & \text { cortical silent period } \\ \text { EEG } & \text { electroencephalography } \\ \text { EMG } & \text { electromyography } \\ \text { FDI } & \text { first dorsal interosseous } \\ \text { GABA } & \text { gamma-aminobutyric acid } \\ \text { ICC } & \text { intraclass correlation coefficient } \\ \text { ICF } & \text { intracortical facilitation } \\ \text { IEDs } & \text { interictal epileptiform discharges } \\ \text { ISI } & \text { interstimulus interval } \\ \text { LICI } & \text { long intracortical inhibition } \\ \text { LTP } & \text { long-term potentiation } \\ \text { MEP } & \text { motor evoked potential } \\ \text { MRI } & \text { magnetic resonance imaging } \\ \text { NMDA } & \text { N-methyl-D-aspartate } \\ \text { NREM } & \text { non-rapid eye movement } \\ \text { PCA } & \text { principal component analysis } \\ \text { PP-ER } & \text { phase periods of consecutive equal responses } \\ \text { rMT } & \text { resting motor threshold } \\ \text { SD } & \text { standard deviation } \\ \text { SEM } & \text { standard error of the mean } \\ \text { SICI } & \text { short intracortical inhibition } \\ \text { TEP } & \text { TMS evoked potential } \\ \text { TMS } & \text { transcranial magnetic stimulation } \\ \text { TP-ER } & \text { time periods of consecutive equal responses } \\ \text { TR } & \text { test response } \\ \end{array}$





\section{English summary}

Epilepsy is characterized by the occurrence of epileptic seizures, resulting from an imbalance between excitatory and inhibitory brain activity. Around 5\% of the population experiences an unprovoked seizure during their lifetime. However, less than half of them actually develop epilepsy. Patients are diagnosed with epilepsy when they had two or more seizures or when they had one seizure with an increased risk of recurrence. After a first seizure it is therefore important to estimate the risk of more seizures. Finding epileptiform discharges in the electroencephalogram (EEG) reflects a tendency to generate seizures. However, if no abnormalities are found, uncertainty remains as the sensitivity of EEG recordings is limited. Indeed, in about $10 \%$ of patients with epilepsy, abnormalities are not found despite repeated or prolonged recordings. This motivated our search for a novel biomarker to improve the diagnostic process.

Transcranial magnetic stimulation (TMS) is a technique to assess the balance between excitation and inhibition. It uses magnetic pulses to stimulate the brain. Stimulation consists of giving one pulse at a time (single pulse TMS), or two pulses short after each other (paired pulse TMS). The effect of stimulation can be assessed indirectly using the muscle response (motor evoked potential: MEP), or directly using the brain response (TMS evoked potential: TEP). If the interval between paired pulses is $50-400 \mathrm{~ms}$, the MEPs and TEPs are inhibited (long intracortical inhibition: LICI). In this thesis, all these modalities were combined. We applied single and paired pulse TMS and evaluated the MEP, TEP and LICI. Since epilepsy is associated with increased cortical excitability, we expect the muscle and brain responses to differ from those in people without epilepsy. This thesis describes our first steps towards the implementation of a multimodal TMS approach in epilepsy. It focusses on the clinical feasibility of multimodal TMS (Chapters 2 to 4), on finding biological modulators of cortical excitability (Chapter 5) and on the diagnostic value of multimodal TMS in epilepsy (Chapters 6 to 8).

Chapter 2 evaluates the repeatability of paired pulse TMS, using the muscle response. We measured thirty healthy subjects twice, about a week apart. On a group level repeatability of $\mathrm{LICl}$ was good, whereas individual subjects showed a large variation in $\mathrm{LICI}$ repeatability. In approximately half of the subjects we measured similar $\mathrm{LICI}$ both times, while LICI was (completely) different one week later in the others. This makes it more difficult to follow patients over time using TMS, for example to evaluate the effect of anti-epileptic drugs. 
Chapter 3 evaluates the effect of changes in coil positioning during single and paired pulse TMS, using the muscle and brain response. To give the magnetic pulses, a TMS coil is placed on the head of the subject. The easiest and fastest way to place and hold the coil in position is manually, with a risk that it moves during the session. On a group level, we found no significant effect of a $5 \mathrm{~mm}$ change in coil location on the MEP, TEP or $\mathrm{LICl}$, nor of a $10^{\circ}$ change in orientation on $\mathrm{LICl}$. This indicates that manual coil positioning performed by an experienced investigator is sufficient in clinical practice.

Chapter 4 evaluates the repeatability of single and paired pulse TMS, using the brain response. Similar to the muscle response, repeatability of the TEP was good after one week. In addition, we found a similar distribution pattern for the characteristic components of the single and paired pulse TEP. A difference between both responses was strong inhibition of the late paired pulse TEP components. We used these findings in healthy subjects as reference values for our epilepsy patients.

Chapter 5 explores if infraslow activity modulates cortical excitability. Giving the same magnetic pulse multiple times, not necessarily results in equal MEPs and TEPs. Very slow biological fluctuations may cause responses to vary over time. This large response variability may limit the applicability of TMS. Our findings indicate that infraslow activity contributes to the variability, although other mechanisms are likely involved as well. By only giving pulses during specific periods of the infraslow activity, it might be possible to reduce the response variation.

Chapter 6 provides a systematic overview of the current single and paired pulse TMS findings in epilepsy patients. So far only muscle responses have been evaluated in epilepsy patients who were not (yet) taking any medication. Findings were most consistent for paired pulse TMS. Compared to healthy subjects, cortical excitability was significantly increased in epilepsy patients at the intervals 250 and $300 \mathrm{~ms}$.

Chapter 7 evaluates differences in the muscle response between refractory epilepsy patients and healthy subjects. Around $30 \%$ of the epilepsy patients continues to have seizures, despite taking anti-epileptic drugs. Based on combined $\mathrm{LICl}$ data from four different centers, we could not differentiate refractory epilepsy patients from healthy subjects. We were unable to confirm previous findings of increased cortical excitability in epilepsy patients.

Chapter 8 explores the potential of multimodal TMS to improve the diagnostic process. We measured patients short after their first seizure. Some of them were eventually diagnosed with epilepsy and some not. We could differentiate patients diagnosed with epilepsy from those without epilepsy and from healthy subjects, using $\mathrm{LICl}$. In contrast to previous findings, excitability was increased at an interval of $100 \mathrm{~ms}$ and decreased 
at $200 \mathrm{~ms}$. For single pulse TMS, we found no significant differences between the three subject categories.

In conclusion, we showed the clinical feasibility and potential of multimodal TMS to improve the diagnostic process in epilepsy. 



\section{Nederlandse samenvatting}

Epilepsie wordt gekenmerkt door het krijgen van epileptische aanvallen, als gevolg van een disbalans tussen activerende en remmende hersenactiviteit. Ongeveer $5 \%$ van de bevolking krijgt een niet-uitgelokte aanval tijdens hun leven. Echter, minder dan de helft ontwikkelt ook daadwerkelijk epilepsie. Patiënten worden gediagnosticeerd met epilepsie als ze twee of meer aanvallen hebben gehad, of als ze één aanval hebben gehad met een verhoogd risico op herhaling. Na een eerste aanval is het daarom belangrijk om het risico op meer aanvallen in te schatten. Het vinden van epileptiforme afwijkingen in het elektro-encefalogram (EEG) wijst op een neiging om aanvallen te genereren. Wanneer er echter geen afwijkingen gevonden worden, blijt de onzekerheid omdat de gevoeligheid van het EEG beperkt is. Inderdaad, bij ongeveer $10 \%$ van de epilepsiepatiënten worden geen afwijkingen gevonden, ondanks herhaalde of langdurige EEG registraties. Dit heeft ons gemotiveerd om op zoek te gaan naar een nieuwe biomarker om het diagnostisch proces te verbeteren.

Transcraniële magnetische stimulatie (TMS) is een techniek om de balans tussen activatie en remming te bepalen. Hierbij worden magnetische pulsen gebruikt om de hersenen te stimuleren. Stimulatie bestaat uit het geven van één puls per keer (enkel puls TMS), of twee pulsen kort na elkaar (dubbel puls TMS). Het effect van stimulatie kan indirect worden bepaald met behulp van de spierreactie (motor evoked potential: MEP), of direct met de hersenreactie (TMS evoked potential: TEP). Als het interval tussen dubbele pulsen 50-400 ms is, worden de MEPs en TEPs onderdrukt (long intracortical inhibition: $\mathrm{LICI}$ ). In dit proefschrift zijn al deze modaliteiten gecombineerd. We hebben enkel en dubbel puls TMS toegepast en de MEP, TEP en LICI geëvalueerd. Omdat epilepsie geassocieerd is met een verhoogde prikkelbaarheid van de hersenen, verwachten we dat de spier- en hersenreacties verschillen van die bij personen zonder epilepsie.

Dit proefschrift beschrijft onze eerste stappen richting de implementatie van een multimodale TMS aanpak bij epilepsie. We hebben ons gericht op de klinische haalbaarheid van multimodale TMS (Hoofdstukken 2 tot 4), op het vinden van biologische modulatoren van de prikkelbaarheid (Hoofdstuk 5) en op de diagnostische waarde van multimodale TMS bij epilepsie (Hoofdstukken 6 tot 8 ). 
Hoofdstuk 2 evalueert de herhaalbaarheid van dubbel puls TMS, met behulp van de spierreactie. We hebben dertig gezonde personen tweemaal gemeten, met een tussenpoos van ongeveer één week. Op groepsniveau was de herhaalbaarheid van $\mathrm{LICl}$ goed, terwijl individuele proefpersonen een grote variatie in $\mathrm{LICI}$ herhaalbaarheid lieten zien. Bij ongeveer de helft van de personen was $\mathrm{LICl}$ beide keren hetzelfde, terwijl LICI een week later (compleet) anders was bij de rest. Dit maakt het lastiger om patiënten over de tijd te volgen met TMS, bijvoorbeeld om het effect van epilepsie medicatie te evalueren.

Hoofdstuk 3 evalueert het effect van veranderingen in de spoel positionering tijdens enkel en dubbel puls TMS, met behulp van de spier- en hersenreactie. Om de magnetische pulsen te geven, wordt een TMS spoel op het hoofd van de proefpersoon geplaatst. Het eenvoudigste en snelste is om de spoel handmatig te plaatsen en vast te houden, met als risico dat deze verplaatst gedurende de meting. Op groepsniveau vonden we geen significant effect van een $5 \mathrm{~mm}$ verandering in spoel locatie op de MEP, TEP en $\mathrm{LICl}$, noch van een $10^{\circ}$ verandering in oriëntatie op $\mathrm{LICl}$. Dit geeft aan dat het handmatig positionering van de spoel door een ervaren onderzoeker volstaat in de klinische praktijk.

Hoofdstuk 4 evalueert de herhaalbaarheid van enkel en dubbel puls TMS, met behulp van de hersenreactie. Vergelijkbaar met de spierreactie, was de herhaalbaarheid van de TEP goed een week later. Daarnaast vonden we een vergelijkbaar verspreidingspatroon voor de karakteristieke componenten van de enkel en dubbel puls TEP. Een verschil tussen beide reacties was een sterke onderdrukking van de late dubbel puls TEP componenten. Deze bevindingen bij gezonde personen hebben we gebruikt als referentiewaarden voor onze epilepsiepatiënten.

Hoofdstuk 5 onderzoekt of infraslow activiteit de prikkelbaarheid van de hersenen moduleert. Het meerdere keren achter elkaar geven van dezelfde magnetische puls, resulteert niet per definitie in gelijke MEPs en TEPs. Zeer trage biologische schommelingen zorgen mogelijk voor variaties in de reacties over de tijd. Deze grote variabiliteit kan de toepasbaarheid van TMS beperken. Onze bevindingen laten zien dat infraslow activiteit bijdraagt aan de variabiliteit, hoewel andere mechanismen waarschijnlijk ook betrokken zijn. Door alleen pulsen te geven op specifieke momenten van de infraslow activiteit, is het wellicht mogelijk om de variatie in reacties te verminderen.

Hoofdstuk 6 geeft een systematisch overzicht van de huidige enkel en dubbel puls TMS bevindingen bij epilepsiepatiënten. Tot nu toe zijn alleen spierreacties onderzocht bij patiënten die (nog) geen medicatie slikten. Bevindingen waren het meest consistent voor dubbel puls TMS. Vergeleken met gezonde personen, was de prikkelbaarheid significant verhoogd bij epilepsiepatiënten bij de intervallen 250 en 300 ms. 
Hoofdstuk 7 evalueert verschillen in de spierreactie tussen refractaire epilepsiepatiënten en gezonde personen. Ongeveer 30\% van de epilepsiepatiënten blijft aanvallen houden, ondanks het gebruik van epilepsie medicatie. Op basis van gecombineerde $\mathrm{LICl}$ data van vier verschillende centra, konden we patiënten met refractaire epilepsie niet onderscheiden van gezonde personen. We waren niet in staat om eerdere bevindingen van een verhoogde prikkelbaarheid bij epilepsiepatiënten te bevestigen.

Hoofdstuk 8 onderzoekt de potentie van multimodale TMS om het diagnostisch proces te verbeteren. We hebben patiënten gemeten kort na hun eerste aanval. Sommigen werden uiteindelijk gediagnosticeerd met epilepsie en sommigen niet. Met behulp van $\mathrm{LICl}$, konden we patiënten met de diagnose epilepsie onderscheiden van patiënten zonder epilepsie en van gezonde personen. In tegenstelling tot eerdere bevindingen, was de prikkelbaarheid verhoogd bij het interval $100 \mathrm{~ms}$ en verlaagd bij $200 \mathrm{~ms}$. Voor enkel puls TMS vonden we geen significante verschillen tussen de drie groepen.

Concluderend hebben we de klinische haalbaarheid en potentie van multimodale TMS aangetoond om het diagnostisch proces bij epilepsie te verbeteren. 



\section{Suomenkielinen yhteenveto}

Epilepsia ilmenee epileptisinä kohtauksina, jotka johtuvat aivojen sähkötoiminnan häiriöistä. Noin 5 prosenttia väestöstä saa elinaikanaan epileptisen kohtauksen vain alle puolelle heistä kehittyy epilepsia. Potilaalla diagnosoidaan epilepsia, jos hänellä on ollut kaksi tai useampia kohtauksia, tai jos hänellä on ollut yksi kohtaus, johon liittyy selvä toistumisen riski. Ensimmäisen kohtauksen jälkeen on tärkeää arvioida riskiä saada uusia kohtauksia. Epilepsiamuotoisten poikkeavuuksien havaitseminen aivosähkökäyrässä (EEG) osoittaa aivojen taipumusta tuottaa kohtauksia. Vaikka aivosähkökäyrässä ei havaittaisi poikkeavuuksia, epävarmuus säilyy EEG-tutkimuksen jälkeen. Kohtaus voi uusia, koska EEG:n kyky havaita poikkeamia on rajallinen. Noin 10 prosentilla epilepsiapotilaista ei havaita poikkeavuuksia toistuvista tai pitkistä EEGmittauksista huolimatta. Nämä ongelmat ovat motivoineet meitä etsimään uutta biomarkkeria diagnoosien parantamiseksi.

Transkraniaalinen magneettistimulaatio (TMS) on tekniikka, jolla pystytään arvioimaan aivojen sähkötoiminnan tasapainoa. TMS:ssä käytetään magneettisia pulsseja aivojen stimuloimiseksi. Stimulaatio sisältää joko yhden pulssin kerrallaan (yksittäispulssiTMS) tai kaksi nopeasti peräkkäin annettavaa pulssia (paripulssi-TMS). Stimulaation vaikutusta voidaan arvioida epäsuorasti käyttämällä lihasvastetta (motor evoked potential: MEP) tai suoraan käyttämällä aivovastetta (TMS evoked potential: TEP). Jos paripulssien väliaika on 50-400 ms, MEP ja TEP ovat inhiboitu (long intracortical inhibition: $\mathrm{LICI}$ ). Tässä väitöskirjassa kaikki nämä modaliteetit yhdistetään. Käytimme yksittäis- ja paripulssi-TMS:ää ja arvioimme MEP:a, TEP:a ja LICI:a. Koska epilepsia liittyy aivojen reaktiivisuuden lisääntymiseen, oletamme että ei-epileptikkojen lihaksen ja aivojen vasteet ovat erilaisia kuin epilepsiapotilailla.

Väitöstutkimuksemme on ensimmäinen askel kohti multimodaalisen TMS:n käyttämistä epilepsiassa. Keskityimme multimodaalisen TMS:n kliiniseen toteutettavuuden (Luvut 2-4), biologisen aivojen reaktiivisuuden modulaattoreiden löytämiseen (Luku 5) ja multimodaalisen TMS:n diagnostiseen käyttöön epilepsiassa (Luvut 6-8).

Luvussa 2 arvioidaan paripulssi-TMS:n toistettavuutta käyttämällä apuna lihasvastetta. Mittasimme kolmekymmentä tervettä henkilöä kahdesti, noin viikon välein. Ryhmässä LICI:n toistettavuus oli hyvä, mutta yksilöiden välillä oli suurta vaihtelua LICItoistettavuudessa. Noin puolella henkilöistä LICl oli molemmilla kerroilla samanlainen, 
noin puolella taas LICl oli (täysin) erilainen viikkoa myöhemmin. Tämä vaikeuttaa potilaiden pitkäaikaista seurantaa TMS:llä, esimerkiksi epilepsialääkkeiden vaikutusten arvioimiseksi.

Luvussa 3 arvioidaan kelan asettamisen muutoksen vaikutusta yksittäis- ja paripulssiTMS:n aikana käyttämällä apuna lihas- ja aivovastetta. Magneettisten pulssien antamiseksi TMS-kela asetetaan henkilön päätä vasten. Helpoin ja nopein tapa asettaa kela ja pitää sitä paikallaan, on tehdä se käsin. Jolloin on kuitenkin vaara, että kela siirtyy paikoiltaan mittauksen aikana. Ryhmätasolla emme löytäneet merkittävää $5 \mathrm{~mm}: \mathrm{n}$ muutoksen vaikutusta MEP:iin, TEP:iin ja LICl:iin, eikä $10^{\circ}$ :n muutoksen vaikutusta LICl:iin. Tämä osoittaa, että kokeneen tutkijan suorittama kelan asettaminen käsin on riittävän tarkka kliinisessä käytössä.

Luvussa 4 arvioidaan yksittäis- ja paripulssi-TMS:n toistettavuutta aivovasteen avulla. Samoin kuin lihasvasteella TEP:n toistettavuus oli hyvä viikon kuluttua. Lisäksi löysimme samanlaisen jakautumiskuvion yksittäis- ja paripulssi-TEP:n tunnusomaisille komponenteille. Vasteita erotti myöhäisten paripulssi-TEP-komponenttien inhibio. Käytimme terveiden henkilöiden mittaustuloksia viitearvona epilepsiapotilaillemme.

Luvussa 5 tutkitaan, vaikuttaako infraslow-aktivisuus aivojen reaktiivisuuteen. Kun samanlaisia magneettisia pulsseja annetaan useita kertoja peräkkäin, ne eivät välttämättä johda yhtäläisiin MEP:iin ja TEP:iin. Hyvin hitaat biologiset vaihtelut voivat aiheuttaa vasteen vaihtelua ajan myötä ja voivat tällä tavalla rajoittaa TMS:n sovellettavuutta. Tulostemme mukaan infraslow-aktivisuus edistää vaihtelevuutta, vaikka muita mekanismeja on todennäköisesti myös mukana. Ratkaisu vaihtelun vähentämiseen voi olla se, että pulsseja annetaan vain infraslow-aktiivisuuden tietyissä vaiheissa.

Luvussa 6 annetaan yleiskatsaus epilepsiapotilaiden nykyisistä yksittäis- ja paripulssiTMS-havainnoista. Toistaiseksi vain lihasvasteita on tutkittu epilepsiapotilailla, jotka eivät (vielä) käytä lääkkeitä. Paripulssi-TMS:n tulokset olivat johdonmukaisimmat. Terveisiin henkilöihin verrattuna aivojen reaktiivisuus lisääntyi merkittävästi epilepsiapotilailla välein 250 ja 300 ms.

Luvussa 7 arvioidaan lihasvasteiden eroja hoitoon huonosti reagoivien epilepsiapotilaiden ja terveiden henkilöiden välillä. Noin 30 prosentilla epilepsiapotilaista on kohtauksia, vaikka he käyttävät epilepsialääkkeitä. Käyttämällä yhdistettyjä LICItietoja neljästä eri keskuksesta, huonosti hoitoon reagoivia epilepsiapotilaita ei voinut erottaa terveistä henkilöistä. Emme pystyneet vahvistamaan aikaisempia havaintoja lisääntyneestä aivojen reaktiivisuudesta epilepsiapotilailla.

Luvussa 8 tutkitaan multimodaalisen TMS:n mahdollisuuksia parantaa diagnoosiprosessia. Mittasimme potilaita pian ensimmäisen kohtauksen jälkeen. Joillakin 
heistä diagnosoitiin myöhemmin epilepsia, mutta ei kaikilla. LICI:n avulla pystyimme erottamaan diagnosoidut potilaat sekä niistä yhden kohtauksen saaneista henkilöistä, joilla ei diagnosoitu epilepsiaa, että terveistä henkilöistä. Vastoin aiempia havaintoja, aivojen reaktiivisuus lisääntyi välein 100 ms, ja laski välein 200 ms. YksittäispulssiTMS:Ilä emme havainneet merkittäviä eroja näiden kolmen ryhmän välillä.

Yhteenvetona toteamme, että multimodaalisen TMS on kliinisesti toteutettavissa ja mahdollinen parantamaan epilepsian diagnosointiprosessia. 



\section{Dankwoord}

Exact 5 jaar geleden begon ik aan dit grote avontuur. Met veel plezier kijk ik terug op een leerzame periode van voornamelijk hoogtepunten. De tijd is echt voorbij gevlogen! Ik wil graag iedereen bedanken die heeft bijgedragen aan dit proefschrift.

Michel, bedankt voor je vertrouwen en de vrijheid die je bood om er mijn eigen onderzoek van te maken. Jouw input en feedback tijdens ons wekelijks overleg leverden veel nieuwe ideeën op om mee aan de slag te gaan. De mogelijkheden om mijn werk te presenteren op de vele internationale congressen kan ik erg waarderen. Tot slot wil ik je bedanken voor de kans om verder te werken aan dit onderzoek als postdoc.

Alle epilepsiepatiënten en gezonde proefpersonen, bedankt voor jullie bereidheid om mee te doen aan mijn onderzoek. Jullie vrijwillige deelname vormt de fundering van dit proefschrift.

Prof. Richardson, prof. Boon, dr. Groeneveld, dr. Leijten, prof. Veltink and prof. van Gils, thank you for reviewing my thesis on scientific value and being a member of my graduation committee.

Co-authors of the chapters in this thesis, many thanks for your input and collaboration. Prisca, dankzij jouw doorzettingsvermogen hebben we op een deel van onze vragen antwoord gekregen vanuit Australië. De volledige waarheid zullen we helaas nooit kunnen achterhalen. Gelukkig hebben we alle negatieve energie om weten te zetten in een prachtig artikel in Brain (Hoofdstuk 7) en een internationaal TMS consortium!

KNF laboranten, neurologie-assistenten en neurologen, bedankt voor jullie hulp bij het benaderen van de epilepsiepatiënten. Nu ik niet meer zo vaak ik het ziekenhuis ben, mis ik de gezelligheid tijdens de koffie- en lunchpauzes en de leerzame wetenschapsbesprekingen. Job van der Palen en Marjolein Brusse-Keizer, jullie heldere uitleg heeft enorm geholpen bij het uitvoeren van de statische analyses.

CNPH collega's, ik heb genoten van jullie gezelligheid de afgelopen jaren. Ik stap 's ochtends met veel plezier in de trein naar Enschede, ook al vertrekt deze om 6 uur. Thank you! ECTM en MD\&I collega's, bedankt voor de leuke conversaties in de koffiecorner. Wim, bedankt voor je hulp bij het maken van de noodstopknop en alle perikelen rondom het onderwijs. Edwin van Asseldonk, dankzij jouw Matlabscripts kan ik het TMS apparaat aansturen via de laptop. I would like to thank the audience 
and speakers of the Neuroscience research meeting for their time and contribution. Furthermore, I am grateful to the students I supervised, Anna, Daniël, Irene, Jorn-Jan, Lyan and Michelle, you helped to explore new ideas.

Tanja, ik weet zeker dat het mini symposium net zo goed geregeld zal zijn als de vakgroepuitjes en congressen. Je deur staat altijd open voor nuttig advies of om gewoon even bij te kletsen. Tanja en Jolanda, bedankt voor al het werk dat jullie me uit handen namen! Barry, doordat we tegelijk begonnen, begrepen we elkaar en konden we samen optrekken. Wat was het fijn om iemand naast/tegenover me te hebben om frustraties en successen mee te kunnen delen. Bedankt voor de leuke gesprekken en je hulp bij het onderwijs. Ik wens je veel succes met je nieuwe baan in Amsterdam. Esther, wat was ik blij met al je voorbereidende werk! Ik heb ontzettend genoten van onze samenwerking; wat een mooie artikelen (Hoofdstukken 3 en 6) zijn hieruit voortgekomen. Ondanks je nieuwe baan, ben je nog steeds bereid om mijn TMS vragen te beantwoorden en mijn drafts van kritische feedback te voorzien. Ik wens jou en je gezin alle geluk. Marleen, ik ben dankbaar voor alle hulp die je biedt zonder dat je officieel betrokken bent bij het onderzoek. Niemand is zo vaak proefpersoon geweest tijdens een testmeting als jij. Ik kan altijd bij je terecht voor een kop thee en advies, of het nu gaat om mijn promotie, het begeleiden van studenten of het kopen van een huis. Verder wil jou, Dirk en Marit bedanken voor jullie gastvrijheid. Wat fijn dat er te allen tijde een opgemaakt bed klaarstaat!

CHDR-collega's, in het bijzonder Francis, Jules, Robert-Jan en Titia, bedankt voor jullie interesse, frisse blik en perfecte organisatie. Vanaf het begin waren jullie enthousiast over TMS. Inmiddels loopt alweer de derde TMS studie en staat er een tweede meetopstelling. Titia, wat zou het leuk zijn als deze mooie samenwerking resulteert in een heus farmaco-TMS proefschrift!

Bootcampbuddies, Marieke, Marleen en Tineke, dankzij jullie positieve energie kwam er nét iets sneller een einde aan de vele burpees, squats en lunges. Weer of geen weer, stress of geen stress, jullie zorg(d)en ervoor dat ik met plezier naar de training fiets(te) om na afloop met een voldaan gevoel op de bank te ploffen.

Lieve vrienden, vriendinnen en kennissen, bedankt voor jullie interesse, gezelligheid en de broodnodige afleiding. Carolien, vanaf de peuterspeelzaal zijn we onafscheidelijk. Hééééél erg bedankt voor alle logeerpartijen, vakanties, TLC avonden, handgeschreven brieven en urenlange telefoongesprekken. Jij kent me door en door, een jarenlange vriendschap die ik enorm koester. Emma, Marit en Merel, ook al zien we elkaar niet meer zo vaak als vroeger, toch voelt het altijd weer als vanouds, curieus! Wat leuk dat ons kookclubje nog steeds bestaat en we nog elk jaar samenkomen met onze ouders en aanhang inmiddels. TG Chicks plus anschlüss (de naam wordt steeds erger...), 
na de zeer geslaagde vuurdoop in Limburg heb ik nu al zin in ons weekendje in juli. Lieve Anniek, Laura, Laura, Lidy, Maaike, Marit en Nicole, ook al wonen we inmiddels verspreid over het land toch vinden we voldoende tijd voor eerste-vrijdag-van-demaand-etentjes, Sinterkerst\&Nieuw en weekendjes weg. Mooie tradities, die we hopelijk nog lang in stand kunnen houden. Net als 'samen' BZV kijken op zondagavond; een zeer welkome afleiding tijdens de afrondende fase van mijn proefschrift.

Lieve paranimfen, Carin en Marit, met jullie aan mijn zijde ben ik er helemaal klaar voor. Carin, zonder jouw hulp, ervaring en tomeloze inzet waren de TMS metingen nooit zo soepel verlopen. Maar bovenal, bedankt voor je optimisme en gezelligheid onder werktijd en tijdens congressen, borrels en etentjes. Ik ken niemand met een vastere hand of een lekkerder crème brûlée recept! Marit, vanaf groep 6 tot aan de Master zaten we bij elkaar in de groep/klas/jaar. De verhuizing naar Enschede was een stuk makkelijker met jou om de hoek voor een luisterend oor en een kop thee. Ik wil je heel veel succes wensen met het afronden van je eigen promotie!

Rakkaat mummi, vaari, sukulaiset ja ystävät Suomessa, monet kiitokset teidän kiinnostuksesta työtäni kohtaan väitöskirjan kirjoitamisvaiheessa. Jaksoitte kannustaa minua. Pitkien lomien aikana kesämökillä Savossa sain aina levättyä kunnolla ja pystyin aloittamaan virkistyneenä työt uudelleen. Tiina, kiitokset sinulle, kun autoit minua suomenkielinen yhteenvedon kanssa.

Lieve papa en äiti, jullie lieten me altijd mijn eigen keuzes maken. Bedankt voor jullie onvoorwaardelijke vertrouwen en steun. Nog steeds brengen en halen jullie me op van de bus; wat is het toch fijn om thuis te zijn! Lieve Kaarina, hoewel we elkaar vroeger regelmatig niet konden uitstaan, kunnen we inmiddels niet meer zonder elkaar. Je hebt me geleerd beter naar mijn eigen gevoel te luisteren en hierover te praten. Ik ben zo ontzettend trots op je! Renout, ik kijk altijd uit naar onze hardloop avonturen en je visfoto's natuurlijk :). Of we nu een obtaclerun rennen, rondje Binnenveld met kerst of om het meer in de zomer, samen is het toch echt leuker en makkelijker. Lieve (toekomstige) schoonfamilie, nog voordat Frank en ik een relatie kregen voelde ik me al welkom in Eindhoven. Dat gevoel is alleen maar sterker geworden over de jaren.

Frank, mijn allerliefste, elke dag weer ben ik zo ontzettend blij dat je ruim 10 jaar geleden die eerste cruciale stap hebt gezet. Bedankt voor je eindeloze geduld, rust en steun (ruim meer dan de helft). Ik kijk ontzettend uit naar onze gesprekken op niveau als doctors onder elkaar.

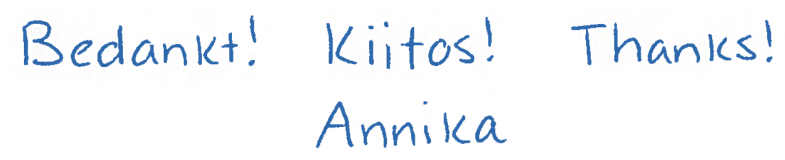





\section{List of publications}

\section{Published}

A.A. de Goede, E.M. ter Braack and M.J.A.M. van Putten. Single and paired pulse transcranial magnetic stimulation in drug naïve epilepsy. Clinical Neurophysiology 2016;127(9): 3140-3155.

A.A. de Goede and M.J.A.M. van Putten. Repeatability of long intracortical inhibition in healthy subjects. Clinical Neurophysiology Practice 2017;2:26-34.

P.R. Bauer, A.A. de Goede, E.M. ter Braack, M.J.A.M. van Putten, R.D. Gill and J.W. Sander. Transcranial magnetic stimulation as a biomarker for epilepsy. Brain 2017;140(3):e18.

A.A. de Goede, B.G. Loef, A.C. Reidinga and A. Schaafsma. Fluid resuscitation in septic patients improves systolic but not diastolic middle cerebral artery flow velocity. Ultrasound in Medicine and Biology 2017;43(11):2591-2600.

P.R. Bauer, A.A. de Goede, W.M. Stern, A.D. Pawley, F.A. Chowdhury, R.M. Helling, et al. Long-interval intracortical inhibition as biomarker for epilepsy: a transcranial magnetic stimulation study. Brain 2018;141(2):409-421.

A.A. de Goede, E.M. ter Braack and M.J.A.M. van Putten. Accurate coil positioning is important for single and paired pulse TMS on the subject level. Brain Topography 2018;31(6):917-930.

E.M. ter Braack, A.A. de Goede and M.J.A.M. van Putten. Resting motor threshold, MEP and TEP variability during daytime. Brain Topography 2019:32(1):17-27.

\section{Submitted}

A.A. de Goede, I. Cumplido Mayoral and M.J.A.M. van Putten. Spatiotemporal dynamics of single and paired pulse TMS-EEG responses. Submitted in revised form: Frontiers in Neuroscience.

A.A. de Goede and M.J.A.M. van Putten. Infraslow activity as a potential modulator of cortical excitability. Submitted in revised form: Journal of Neurophysiology. 



\section{Conference contributions}

\section{Oral presentations}

TMS-EEG bij epilepsie. Winterbijeenkomst Nederlandse Vereniging voor Klinische Neurofysiologie, January 2015, Amsterdam.

Paired pulse TMS-EMG and TMS-EEG in epilepsy. $24^{\text {th }}$ ANT Burgundy Neuromeeting, January 2015, Beaune.

Reproducibility of paired pulse transcranial magnetic stimulation in healthy subjects. KNF Perifeer - KNF Technologie, October 2016, Groningen.

Exploring the modulators of cortical excitability. $16^{\text {th }}$ European Congress of Clinical Neurophysiology ECCN, September 2017, Budapest. For abstract see: Clinical Neurophysiology 2017;128(9):e184.

Spatiotemporal dynamics of single and paired pulse TMS-EEG responses in healthy subjects. $31^{\text {st }}$ International Congress of Clinical Neurophysiology ICCN, May 2018, Washington.

\section{Poster presentations}

Paired pulse TMS-EMG-EEG in epilepsy. $10^{\text {th }} \mathrm{NFSI}$ and $2^{\text {nd }}$ BaCi meeting, September 2015, Utrecht.

Paired pulse TMS-EMG and TMS-EEG in epilepsy. $15^{\text {th }}$ European Congress on Clinical Neurophysiology ECCN, October 2015, Brno. For abstract see: Clinical Neurophysiology 2016;127(3):e81.

Reproducibility of the long intracortical inhibition curve in healthy subjects. $6^{\text {th }}$ International Conference on Transcranial Magnetic Stimulation, September 2016, Göttingen. For abstract see: Clinical Neurophysiology 2017;128(3):e88.

Repeatability of long intracortical inhibition in healthy subjects. $70^{\text {th }}$ American Epilepsy Society AES annual meeting, December 2016, Houston. 
